\title{
Synthesis of Tetrahydroisoquinoline Alkaloids and Related Compounds Through the Alkylation of Anodically Prepared $\alpha$-Amino nitriles.
}

Lotfi Benmekhbi, ${ }^{\S}$ Fadila Louafi, ${ }^{\dagger}$ Thierry Roisnel, ${ }^{\ddagger}$ Jean-Pierre Hurvois. ${ }^{\ddagger \star}$

§Laboratoire de Chimie des Matériaux, Faculté des Sciences Exactes, Université de Constantine1, route de Ain El Bey, 25000 Constantine, Algérie

†Unité de Recherche CHEMS, Faculté des Sciences Exactes, Université de Constantine1, route de Ain El Bey, 25000 Constantine, Algérie

†Sciences Chimiques de Rennes, UMR 6226, CNRS-Université de Rennes 1, 2 Avenue Léon Bernard, 35043, Rennes Cedex, France.

\section{Supporting Information}

\section{Table of contents}

S1-S190: Copies of the proton and carbon NMR spectra of derivatives 1-16, (-)norlaudanosine, (+)-salsolidine, (+)-carnegine and (-)-xylopinine.

S12: ORTEP view of derivative (+)-2a.

S23: ORTEP view of derivative $(+)-\mathbf{2 b}$.

S43: ORTEP view of derivative (+)-1f.

S49: ORTEP view of derivative (+)-19.

S152: ORTEP view of derivative (-)-4.

S185: ORTEP view of (-)-xylopinine.

S164-S170: Determination of enantiomeric ratios of (+)-salsolidine.

S171-S181: Determination of enantiomeric ratios (-)-norlaudanosine by proton and carbon NMR. 


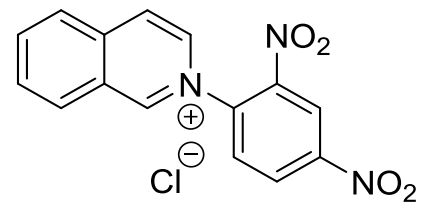

7

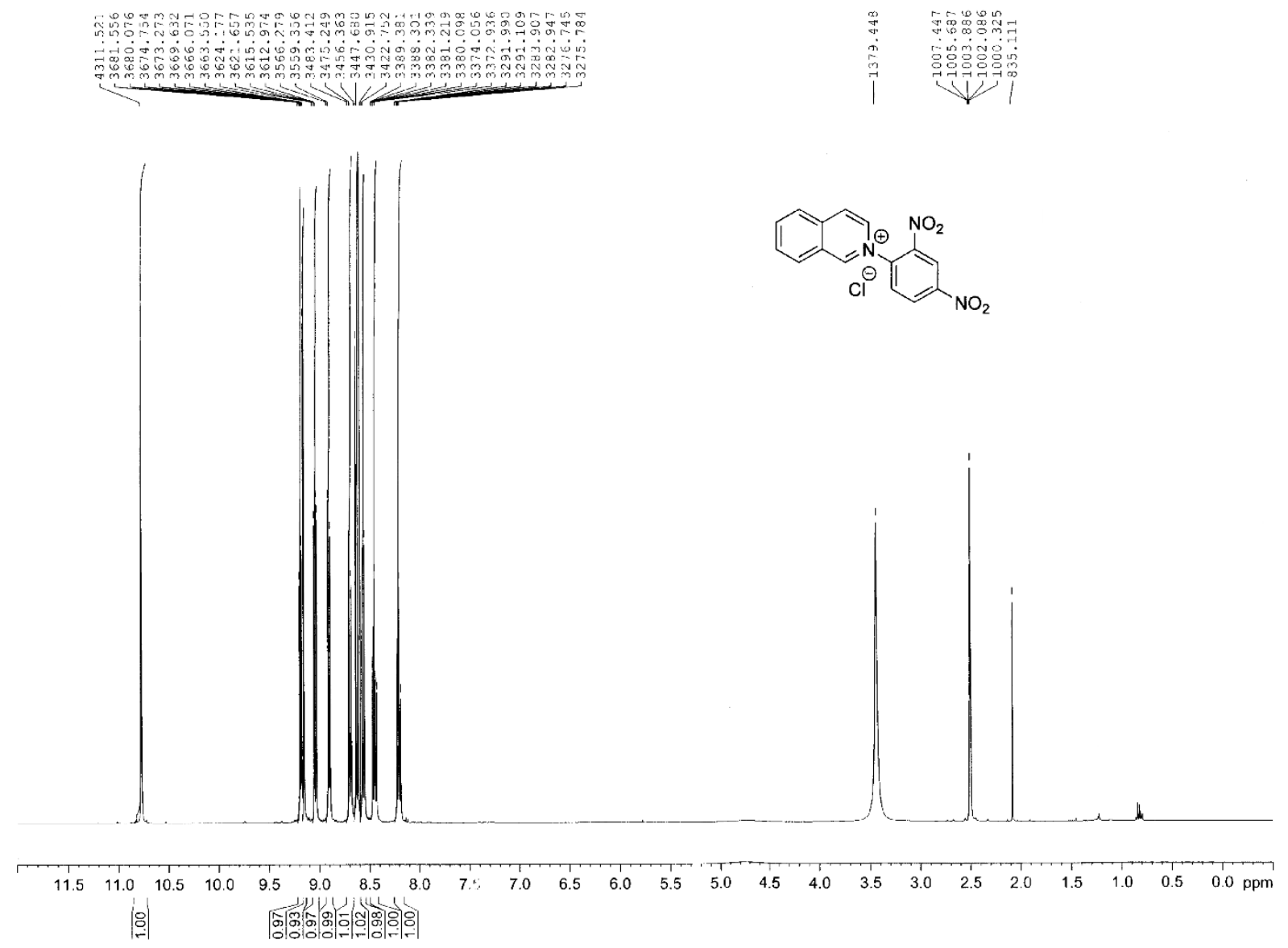

Figure S 1. ${ }^{1} \mathrm{H}$ NMR spectrum (DMSO-d6, $400 \mathrm{MHz}$ ) of derivative 7. 


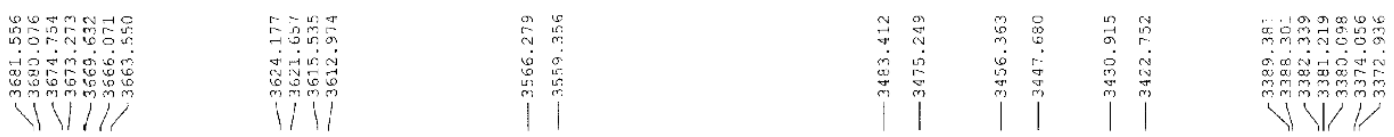

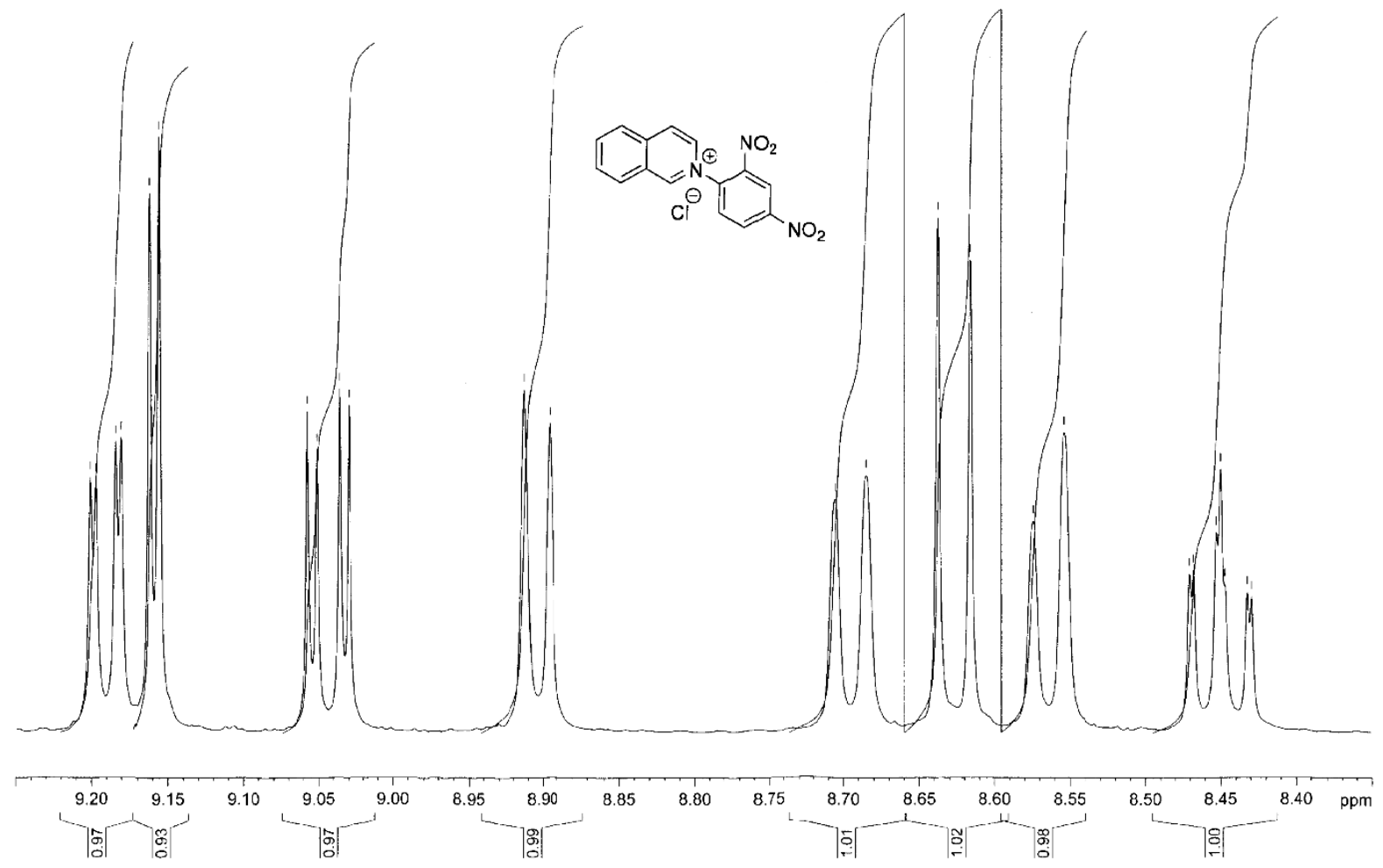

Figure S 2. ${ }^{1} \mathrm{H}$ NMR spectrum (DMSO- $\mathrm{d}_{6}, 400 \mathrm{MHz}$ ) of derivative 7. 


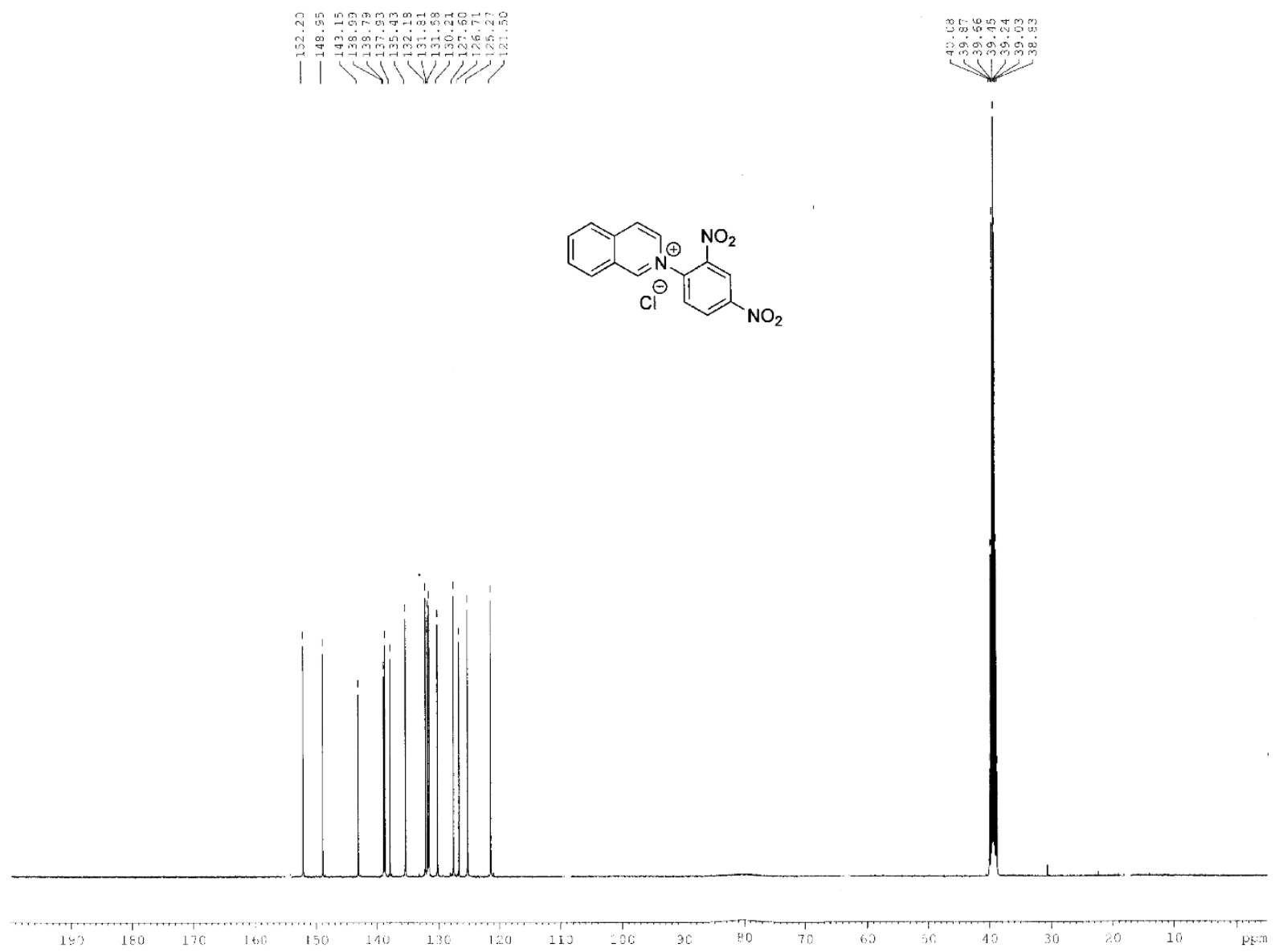

Figure S $3 .{ }^{13} \mathrm{C}$ NMR spectrum (DMSO-d6, $100 \mathrm{MHz}$ ) of derivative 7 . 


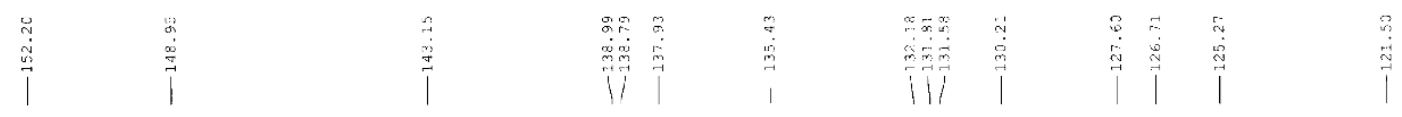
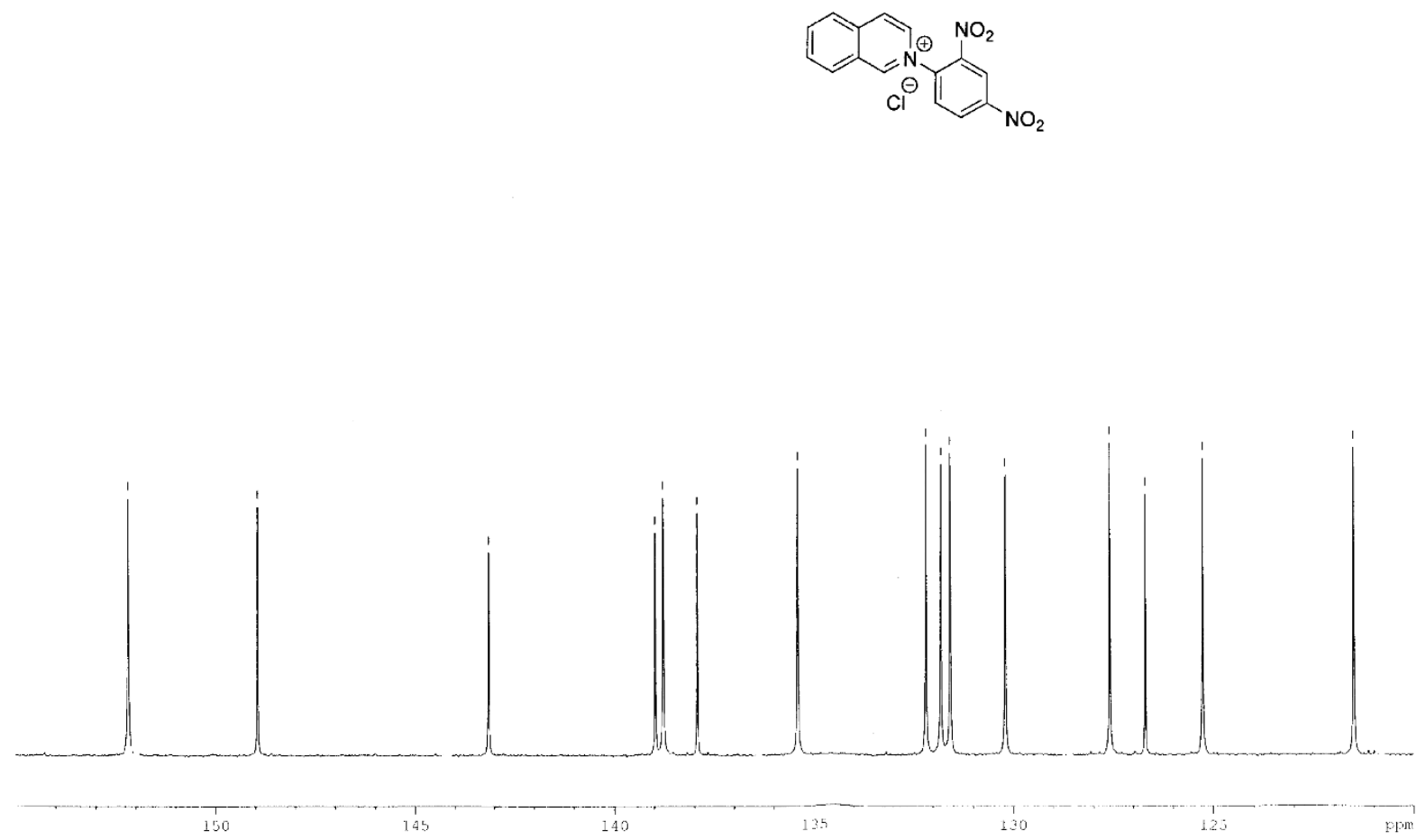

Figure S 4. ${ }^{13} \mathrm{C}$ NMR spectrum (DMSO-d6, $100 \mathrm{MHz}$ ) of derivative 7. 


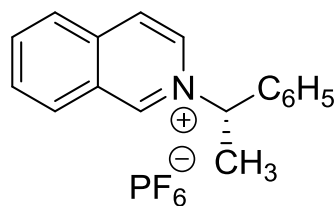

\section{$(-)-8 b$}

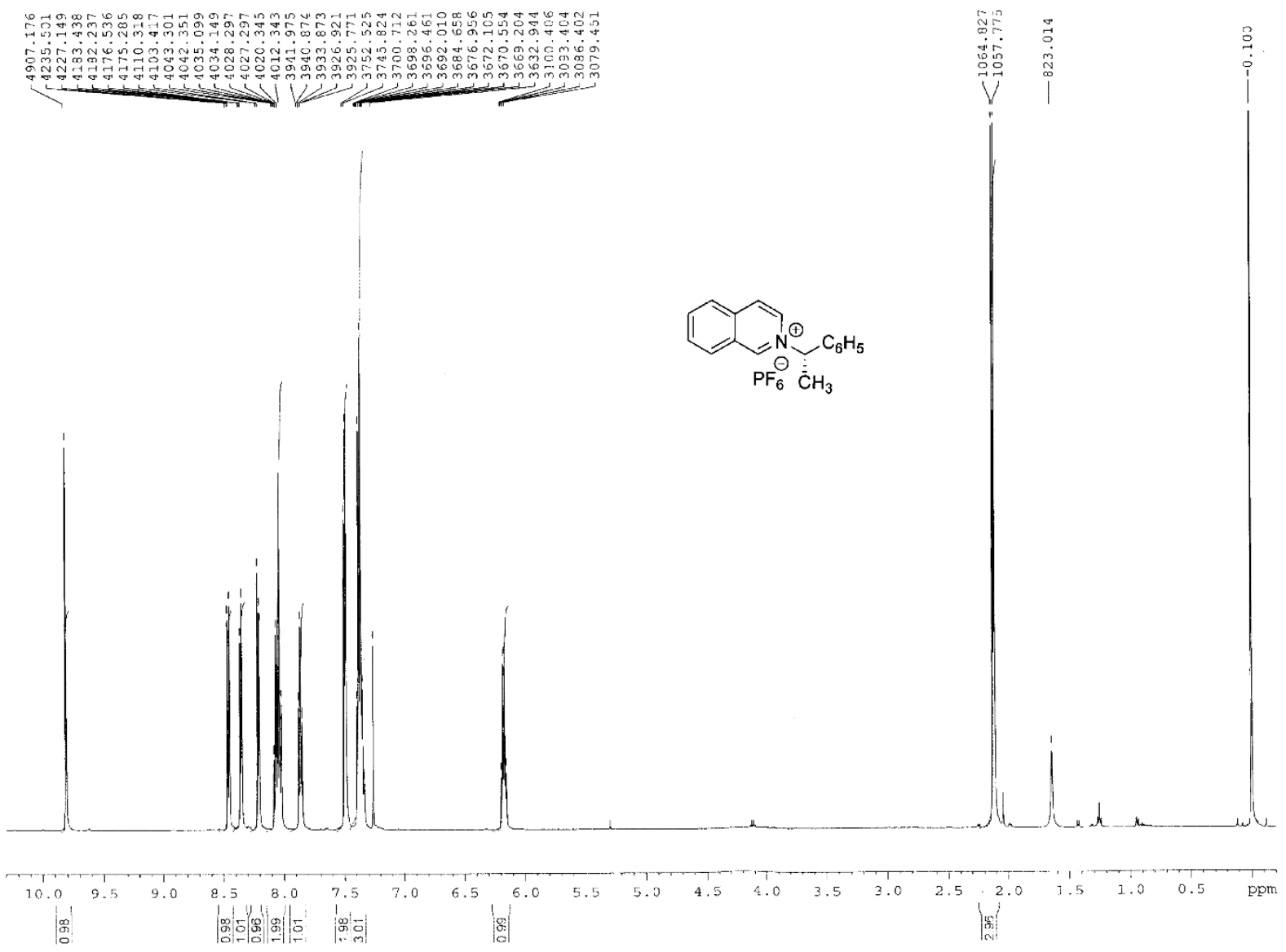

Figure S 5. ${ }^{1} \mathrm{H} \mathrm{NMR}$ spectrum $\left(\mathrm{CDCl}_{3}, 400 \mathrm{MHz}\right)$ of derivative (-)-8b. 


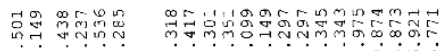

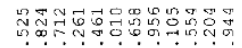

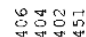

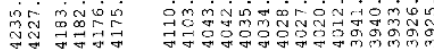

กู่

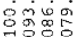

ij

$1 / 4$ Ver

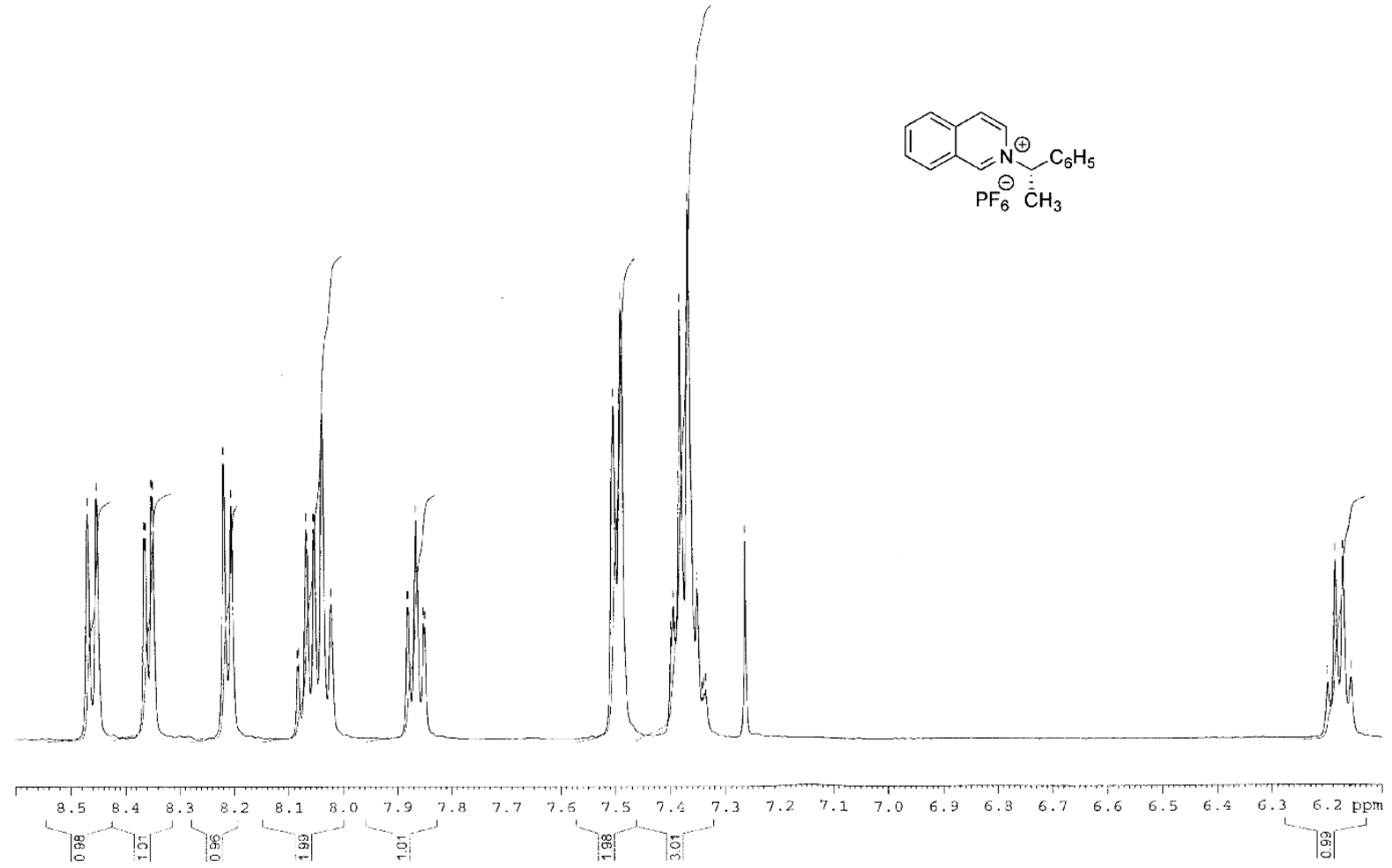

Figure S 6. ${ }^{1} \mathrm{H}$ NMR spectrum $\left(\mathrm{CDCl}_{3}, 400 \mathrm{MHz}\right)$ of derivative (-)-8b. 


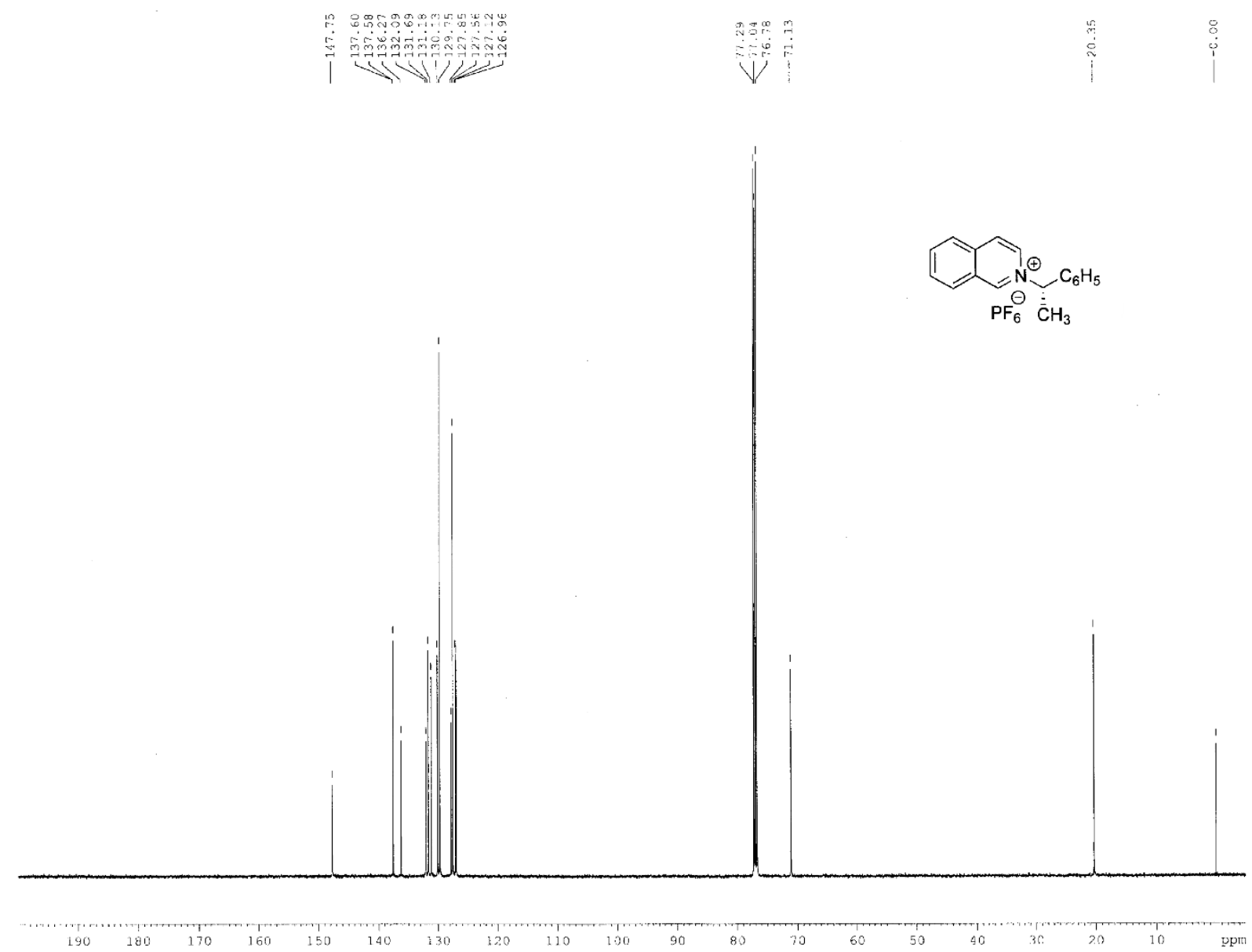

Figure S 7. ${ }^{13} \mathrm{C}$ NMR spectrum $\left(\mathrm{CDCl}_{3}, 100 \mathrm{MHz}\right)$ of derivative (-)-8b. 


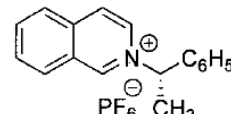

$\mathrm{PF}_{6} \stackrel{\dot{\mathrm{C}} \mathrm{H}_{3}}{ }$

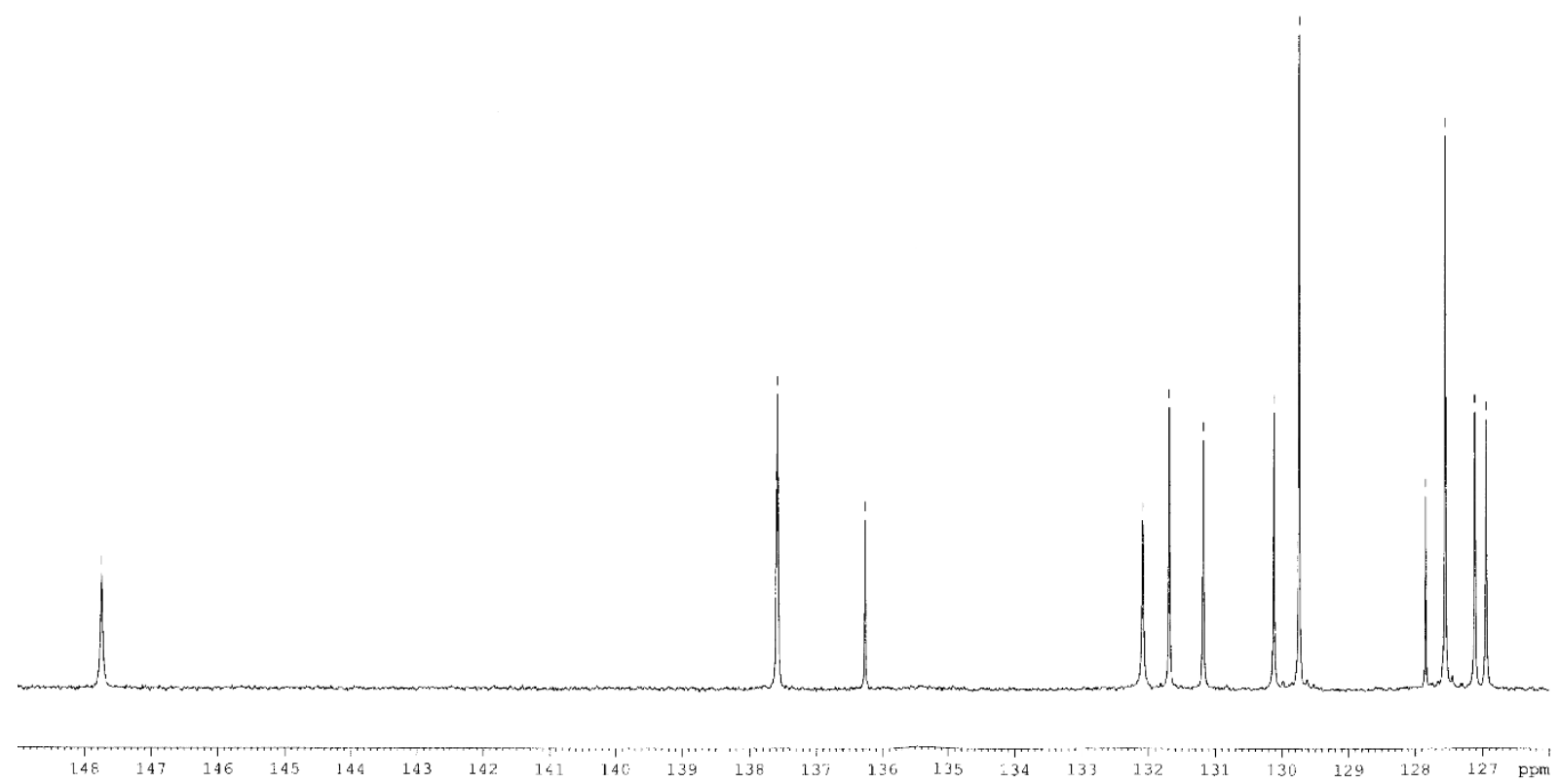

Figure S 8. ${ }^{13} \mathrm{C}$ NMR spectrum $\left(\mathrm{CDCl}_{3}, 100 \mathrm{MHz}\right)$ of derivative (-)-8b. 


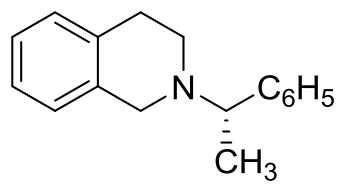

$(+)-6 a$

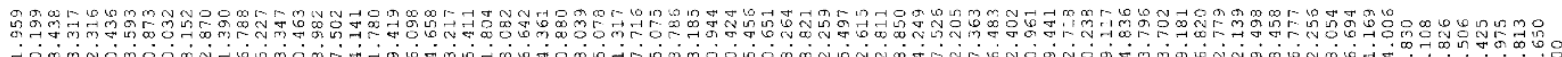

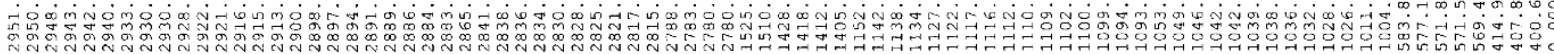

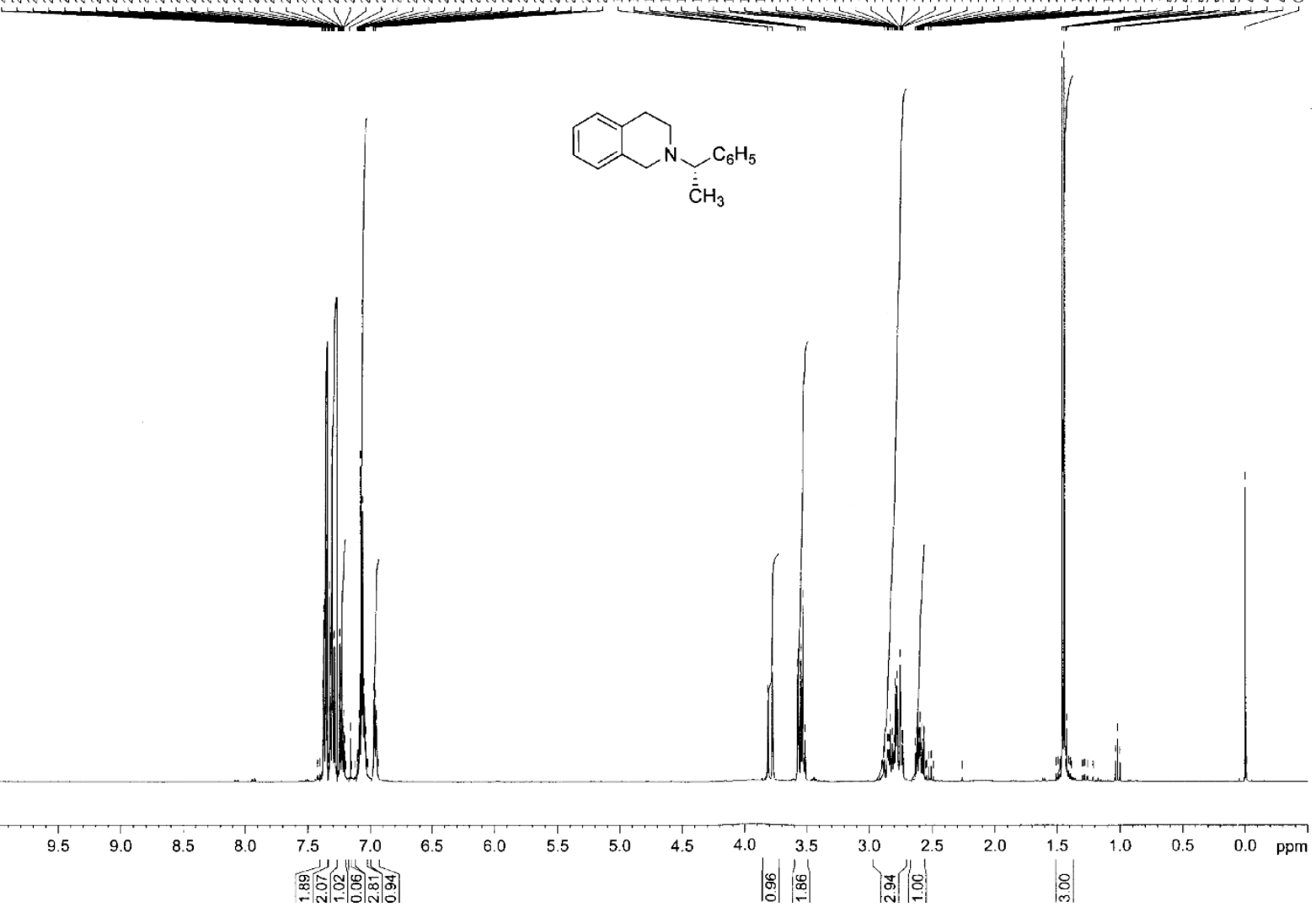

Figure S 9. ${ }^{1} \mathrm{H} \mathrm{NMR}$ spectrum $\left(\mathrm{CDCl}_{3}, 400 \mathrm{MHz}\right)$ of derivative (+)-6a. 


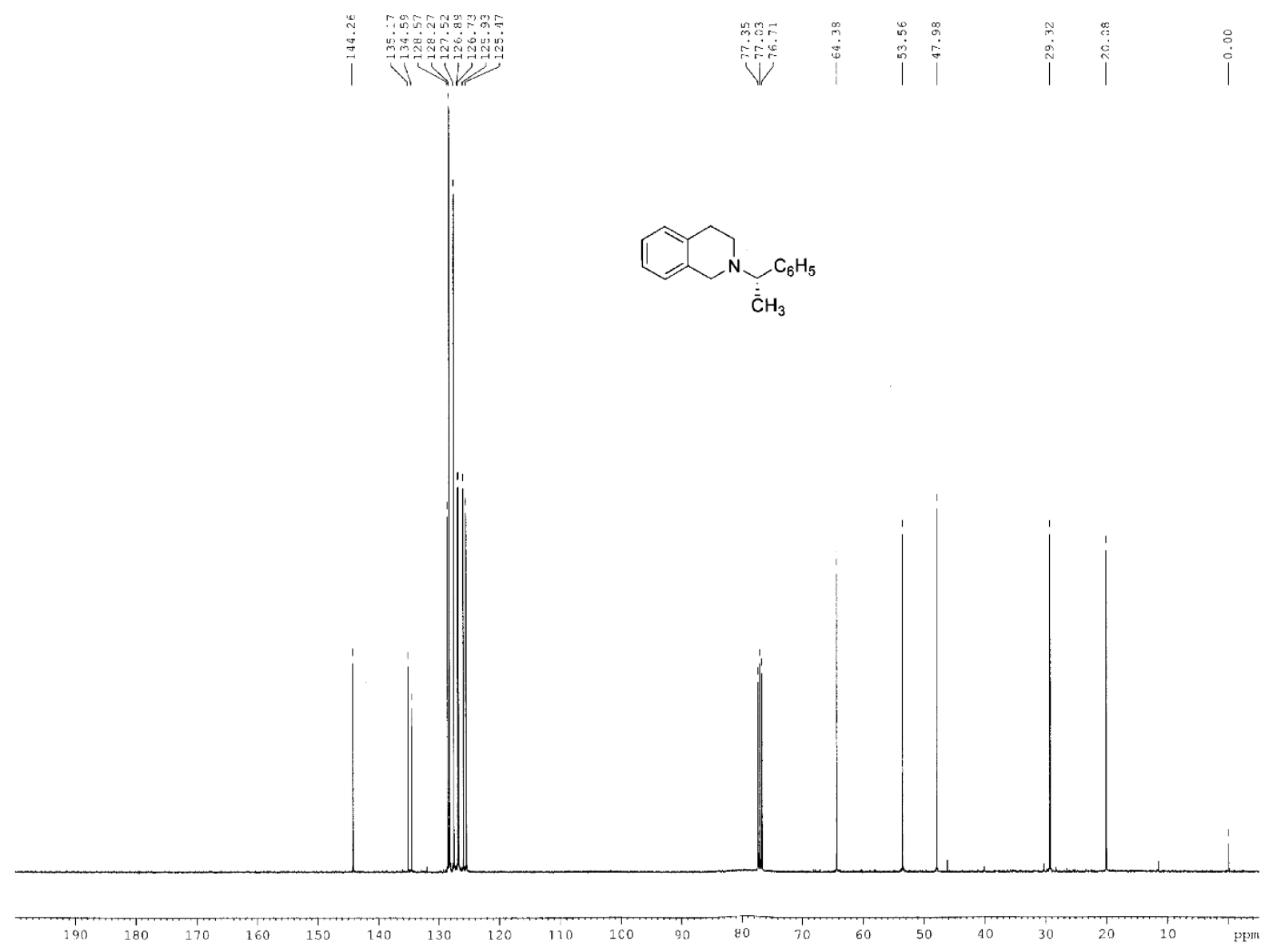

Figure $\mathbf{S} 10 .{ }^{13} \mathrm{C} \mathrm{NMR}$ spectrum $\left(\mathrm{CDCl}_{3}, 100 \mathrm{MHz}\right)$ of derivative (+)-6a. 


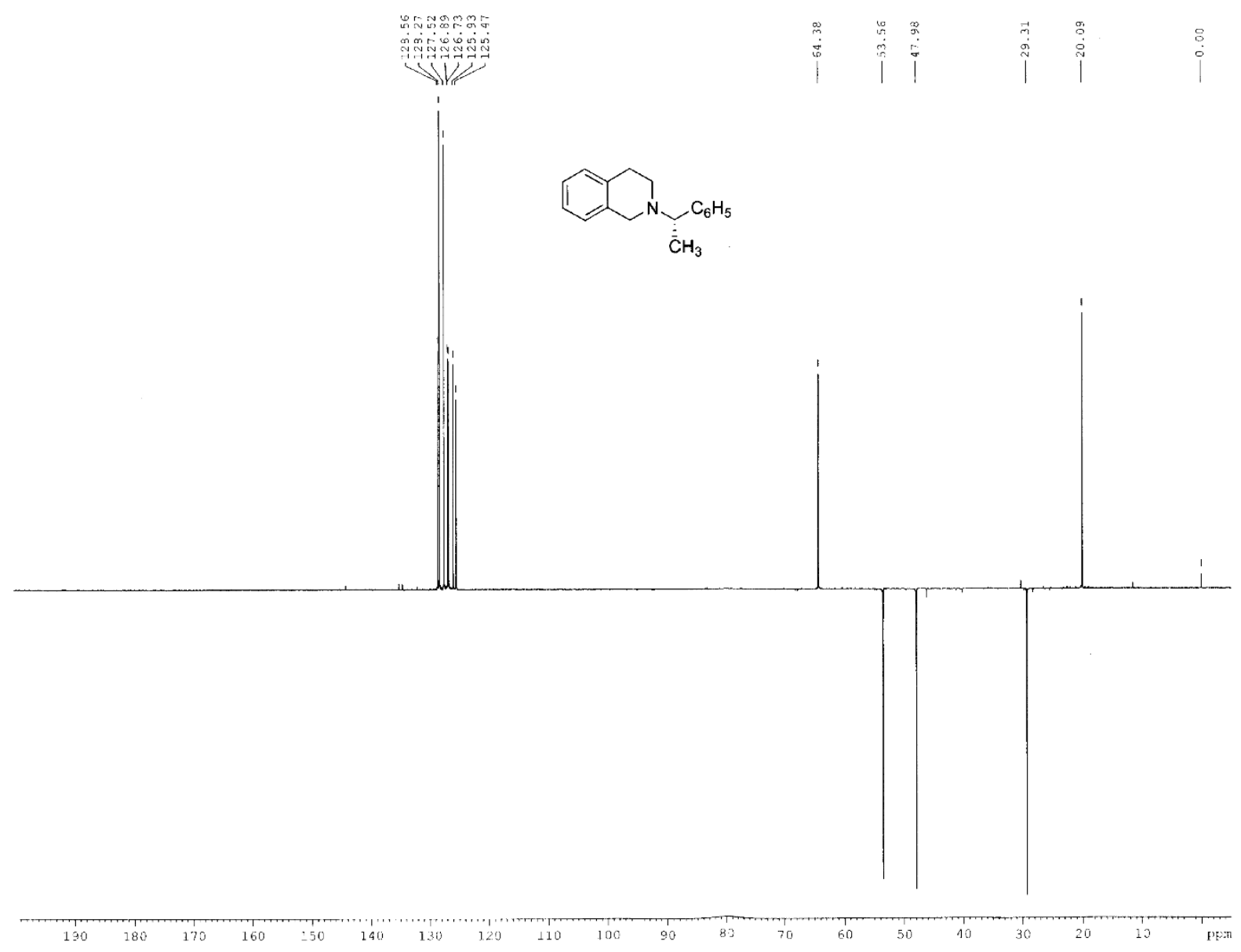

Figure S $11 .{ }^{13} \mathrm{C}$ NMR spectrum $\left(\mathrm{CDCl}_{3}, 100 \mathrm{MHz}\right)$ of derivative (+)-6a. 
S14<smiles>CC(c1ccccc1)N1CCc2ccccc2C1C#N</smiles>

$(+)-2 a$

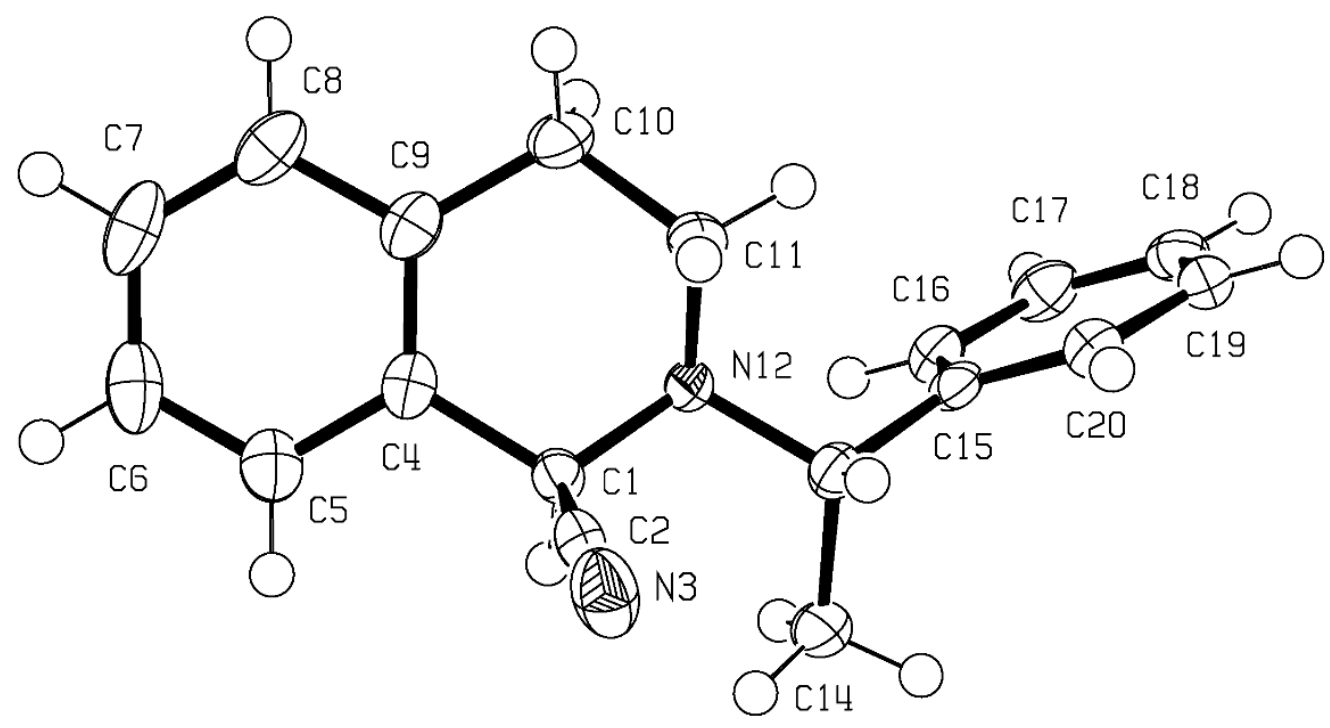

Figure S 12. ORTEP view of $\alpha$-amino nitrile (+)-ra. Ellipsoid plots are drawn with $70 \%$ of probability. 


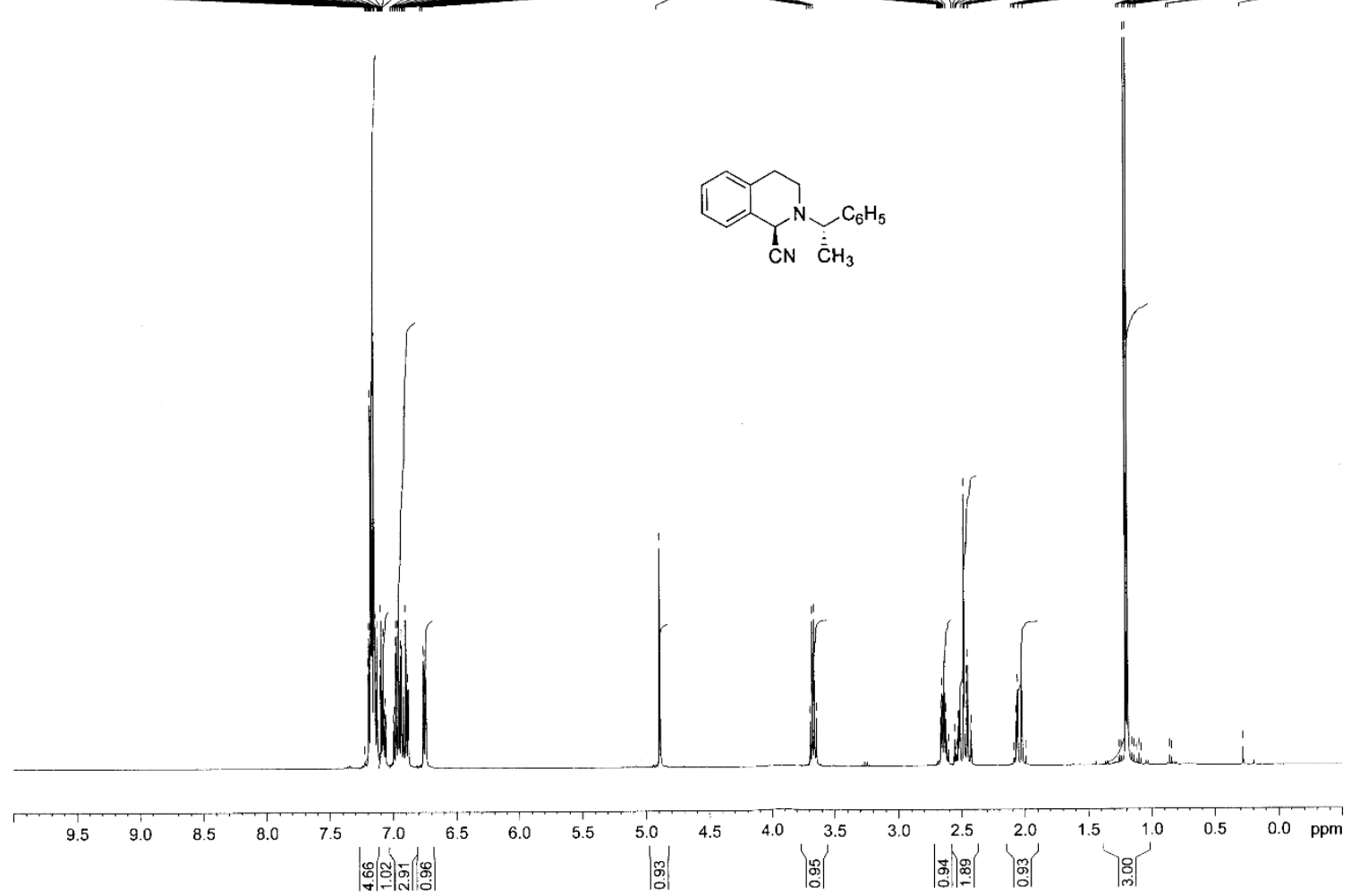

Figure S 13. ${ }^{1} \mathrm{H}$ NMR spectrum $\left(\mathrm{C}_{6} \mathrm{D}_{6}, 400 \mathrm{MHz}\right)$ of derivative (+)-2a. 


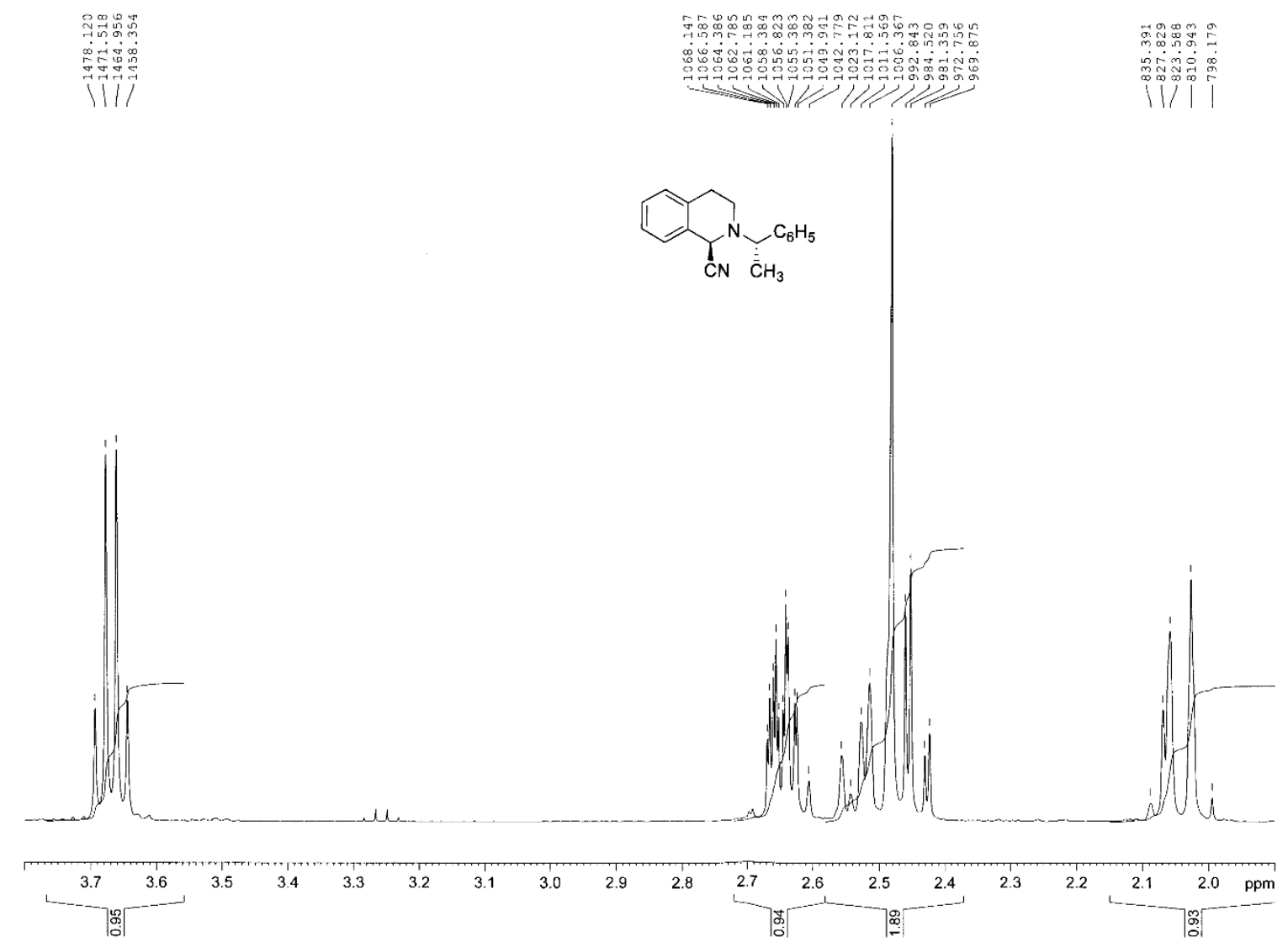

Figure S 14. ${ }^{1} \mathrm{H}$ NMR spectrum $\left(\mathrm{C}_{6} \mathrm{D}_{6}, 400 \mathrm{MHz}\right)$ of derivative (+)-2a. 


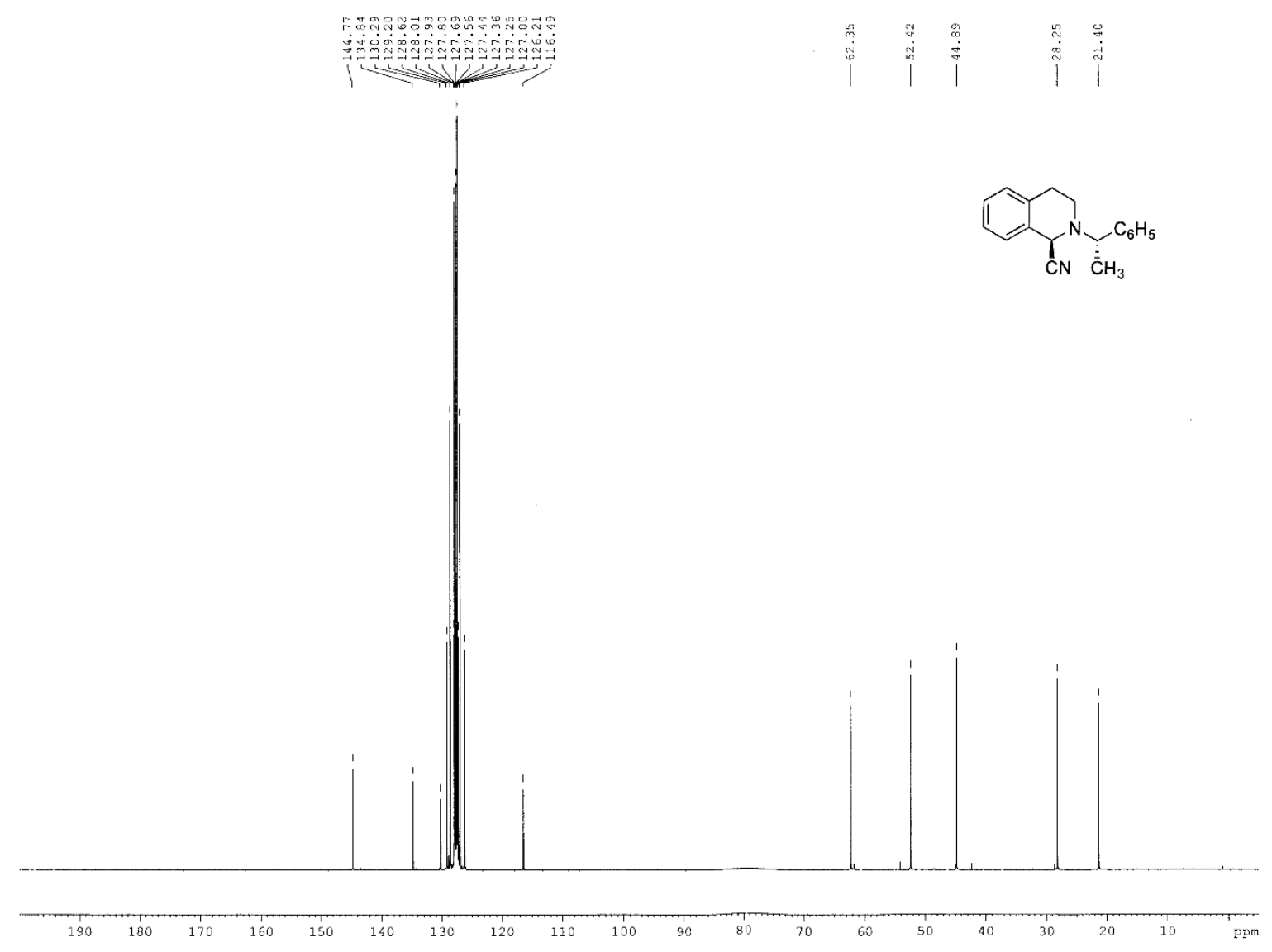

Figure $\mathrm{S} 15 .{ }^{13} \mathrm{C}$ NMR spectrum $\left(\mathrm{C}_{6} \mathrm{D}_{6}, 100 \mathrm{MHz}\right)$ of derivative (+)-2a. 
S18
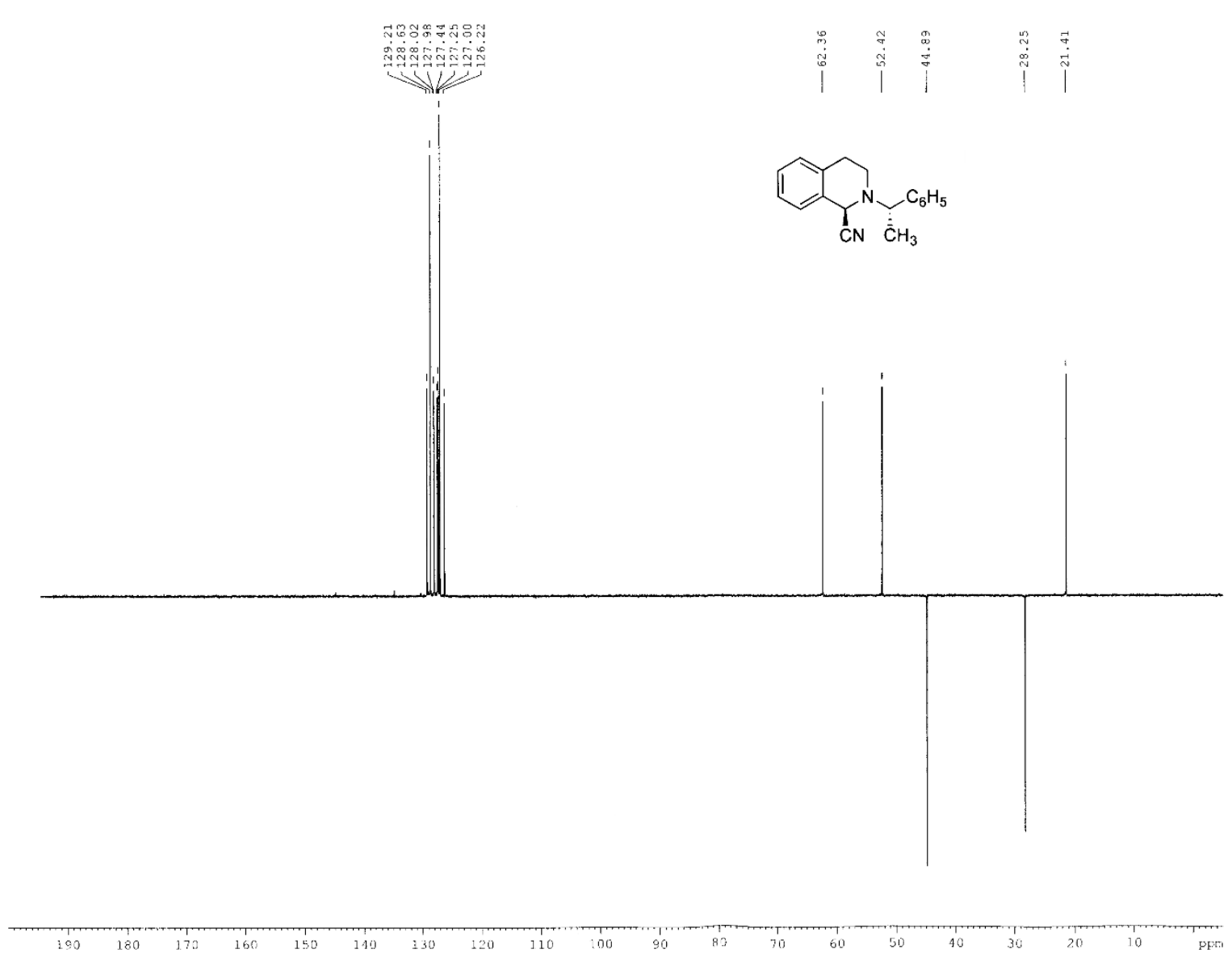

Figure S 16. DEPT C NMR spectrum $\left(\mathrm{C}_{6} \mathrm{D}_{6}, 100 \mathrm{MHz}\right)$ of derivative (+)-ra. 


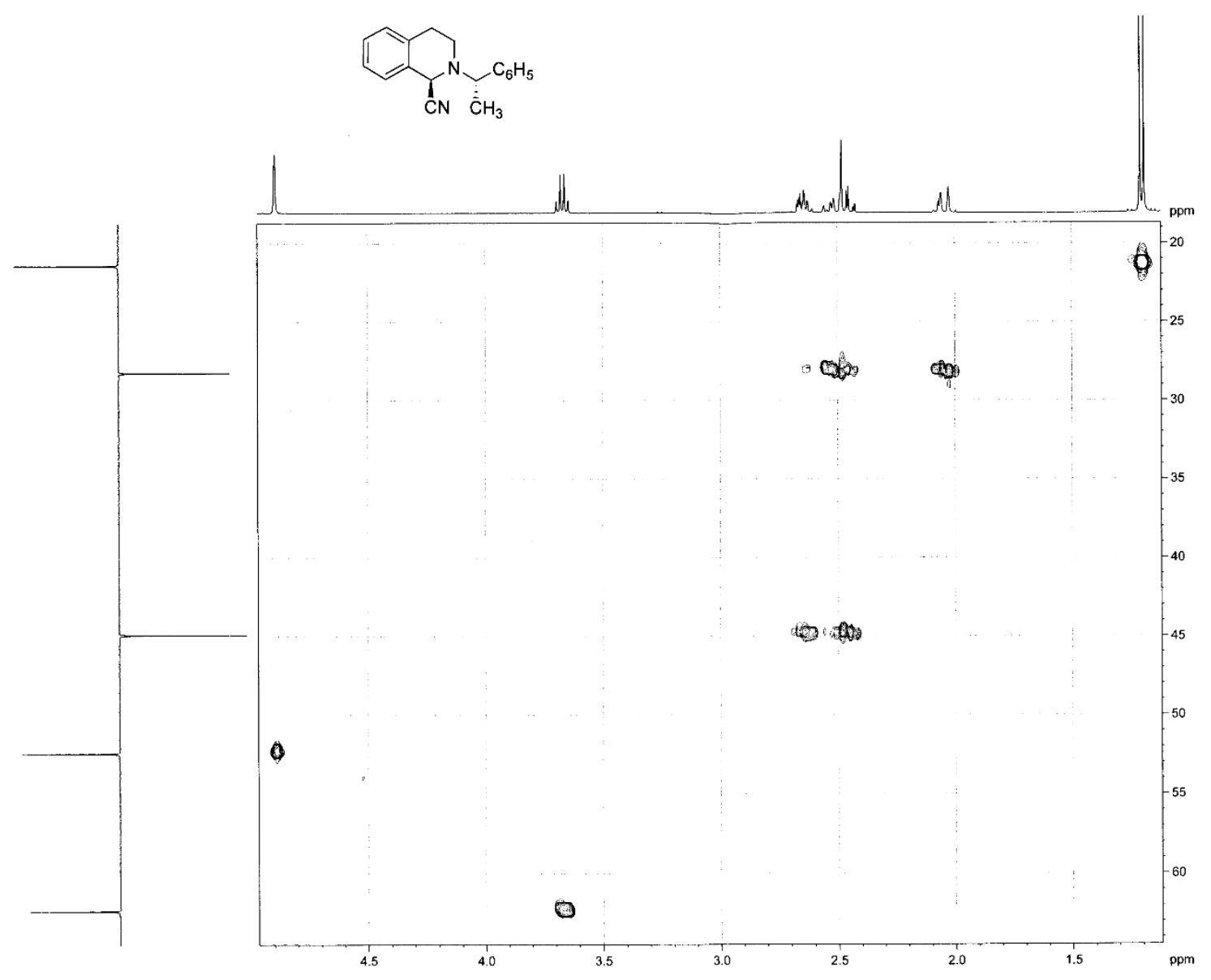

Figure $S$ 17. $H M Q C$ spectrum $\left(C_{6} D_{6}\right)$ of derivative $(+)-2 a$. 
<smiles>CC(c1ccccc1)N1CCc2ccccc2C1C#N</smiles><smiles>CC(C)N1CCc2ccccc2C1C#N</smiles>

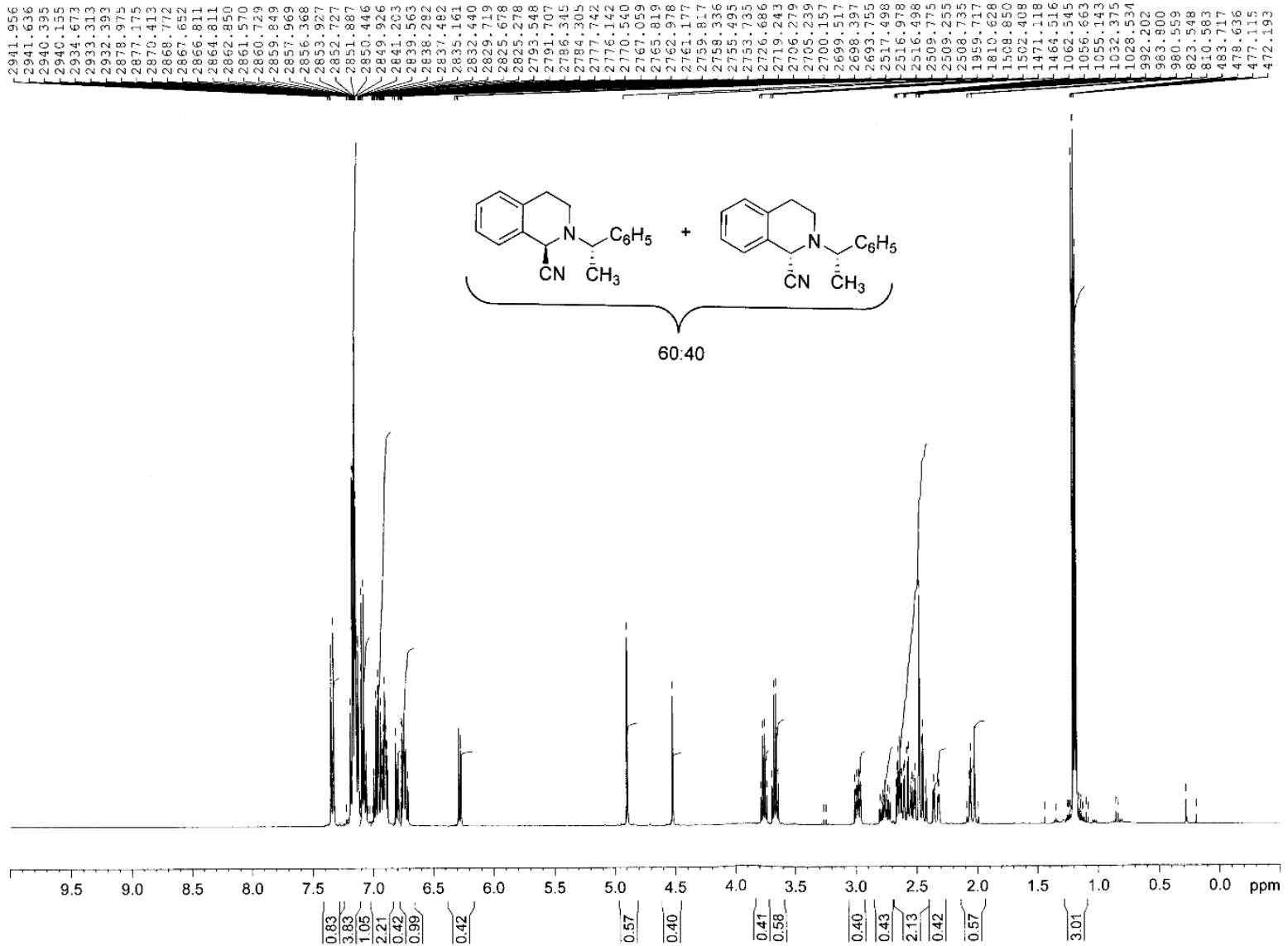

Figure S 18. ${ }^{1} \mathrm{H}$ NMR spectrum $\left(\mathrm{C}_{6} \mathrm{D}_{6}, 400 \mathrm{MHz}\right)$ of derivatives (+)-2a and (-)-2a after a $48 \mathrm{~h}$ standing in $\mathrm{C}_{6} \mathrm{D}_{6}$. 


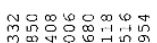

in

(1)
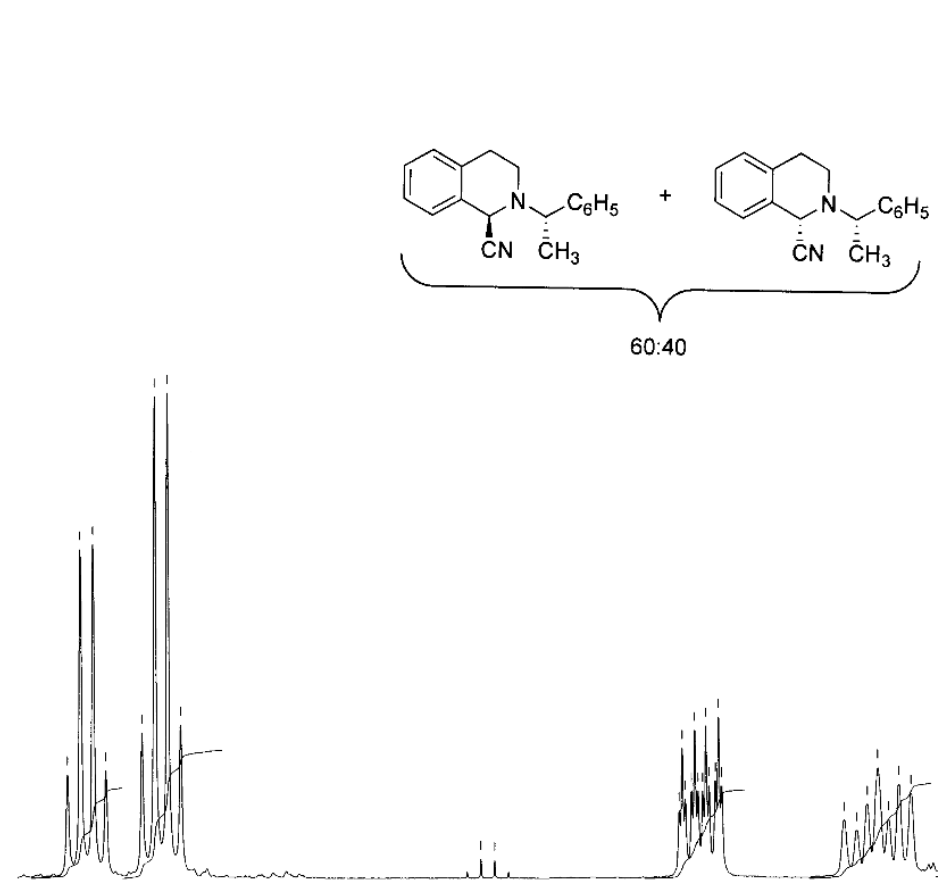

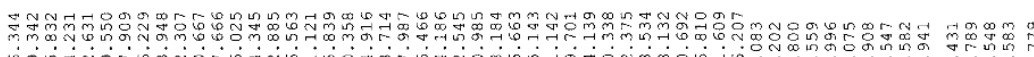

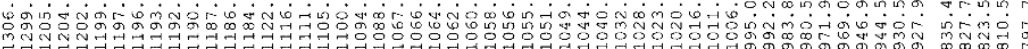

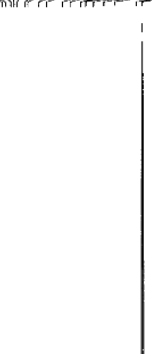

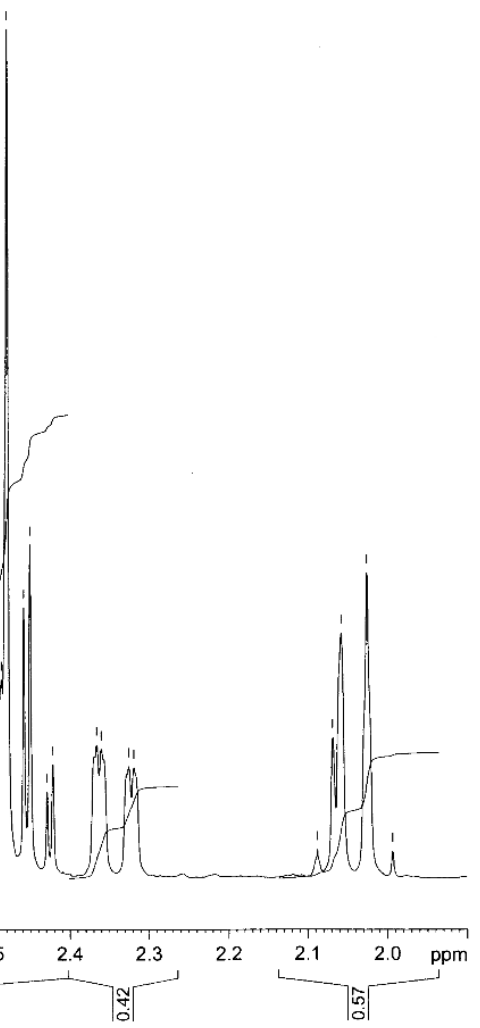

Figure S 19. ${ }^{1} \mathrm{H}$ NMR spectrum $\left(\mathrm{C}_{6} \mathrm{D}_{6}, 400 \mathrm{MHz}\right)$ of derivatives (+)-2a and (-)-2a after a $48 \mathrm{~h}$ standing in $\mathrm{C}_{6} \mathrm{D}_{6}$. 


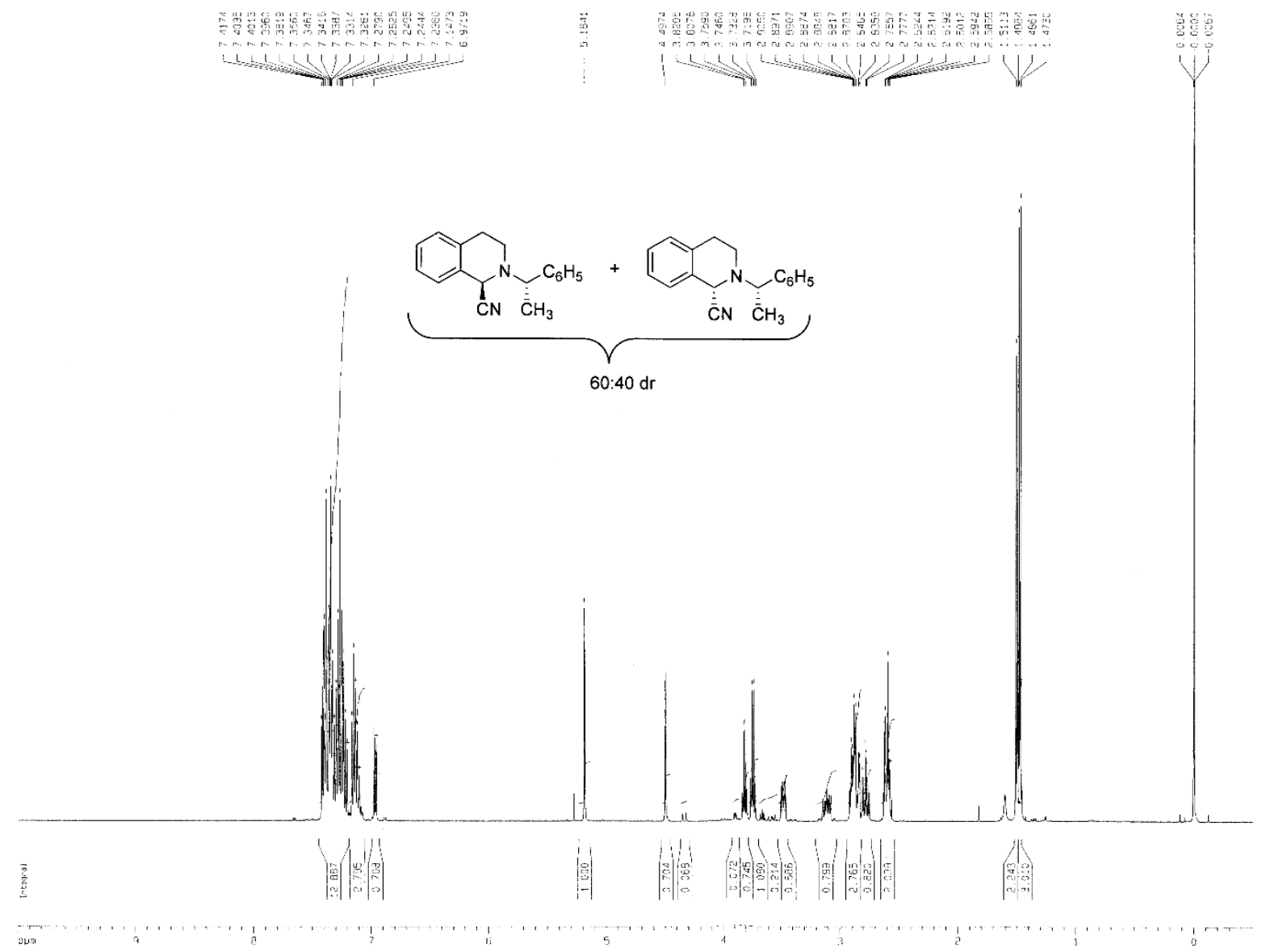

Figure S 20. ${ }^{1} \mathrm{H}$ NMR spectrum $\left(\mathrm{CDCl}_{3}, 500 \mathrm{MHz}\right)$ of derivatives (+)-2a and (-)-2a. 
S23

咅
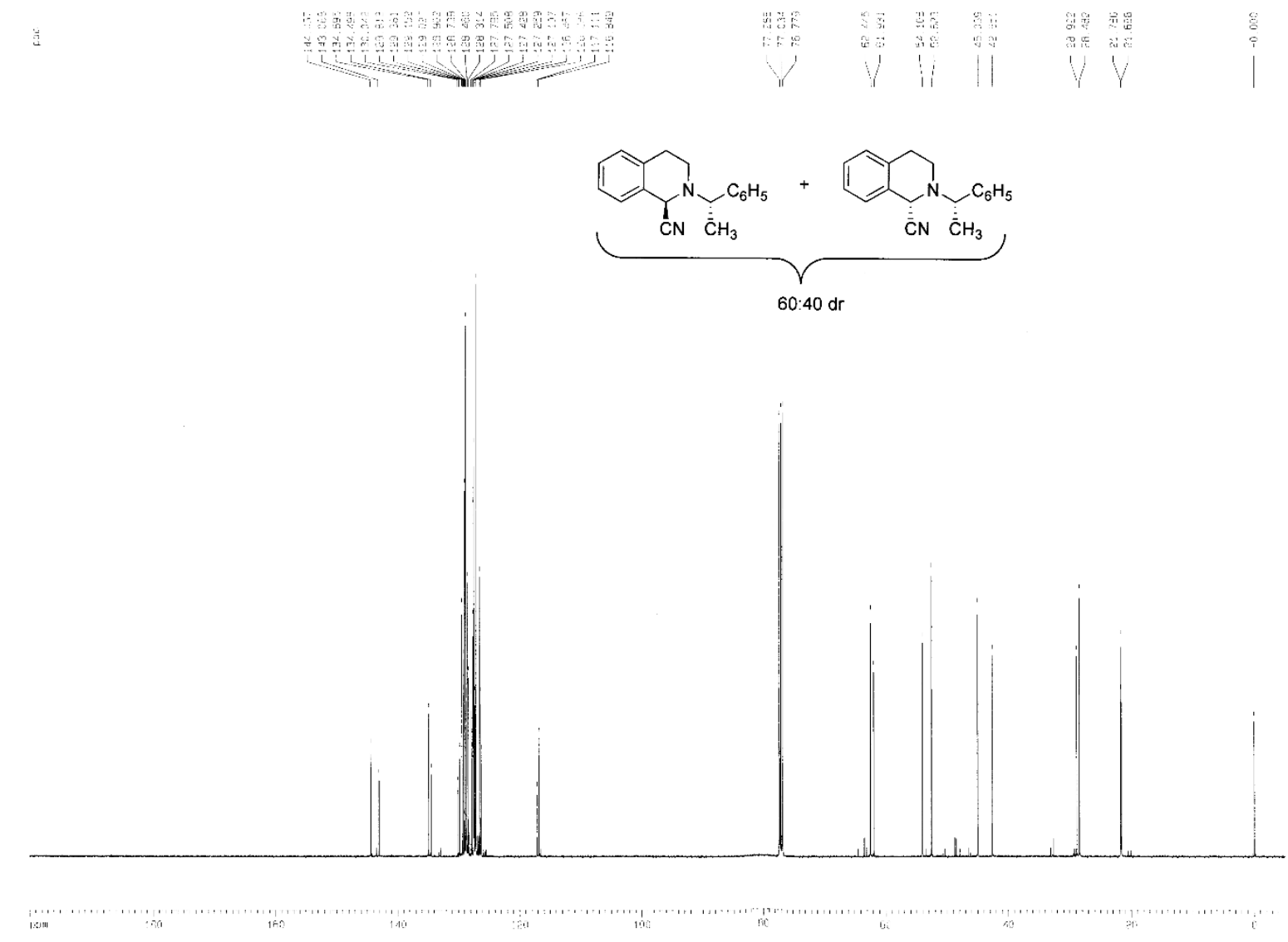

Figure S 21. ${ }^{13} \mathrm{C}$ NMR spectrum $\left(\mathrm{CDCl}_{3}, 125 \mathrm{MHz}\right)$ of derivatives (+)-2a and (-)-2a. 
S24

点
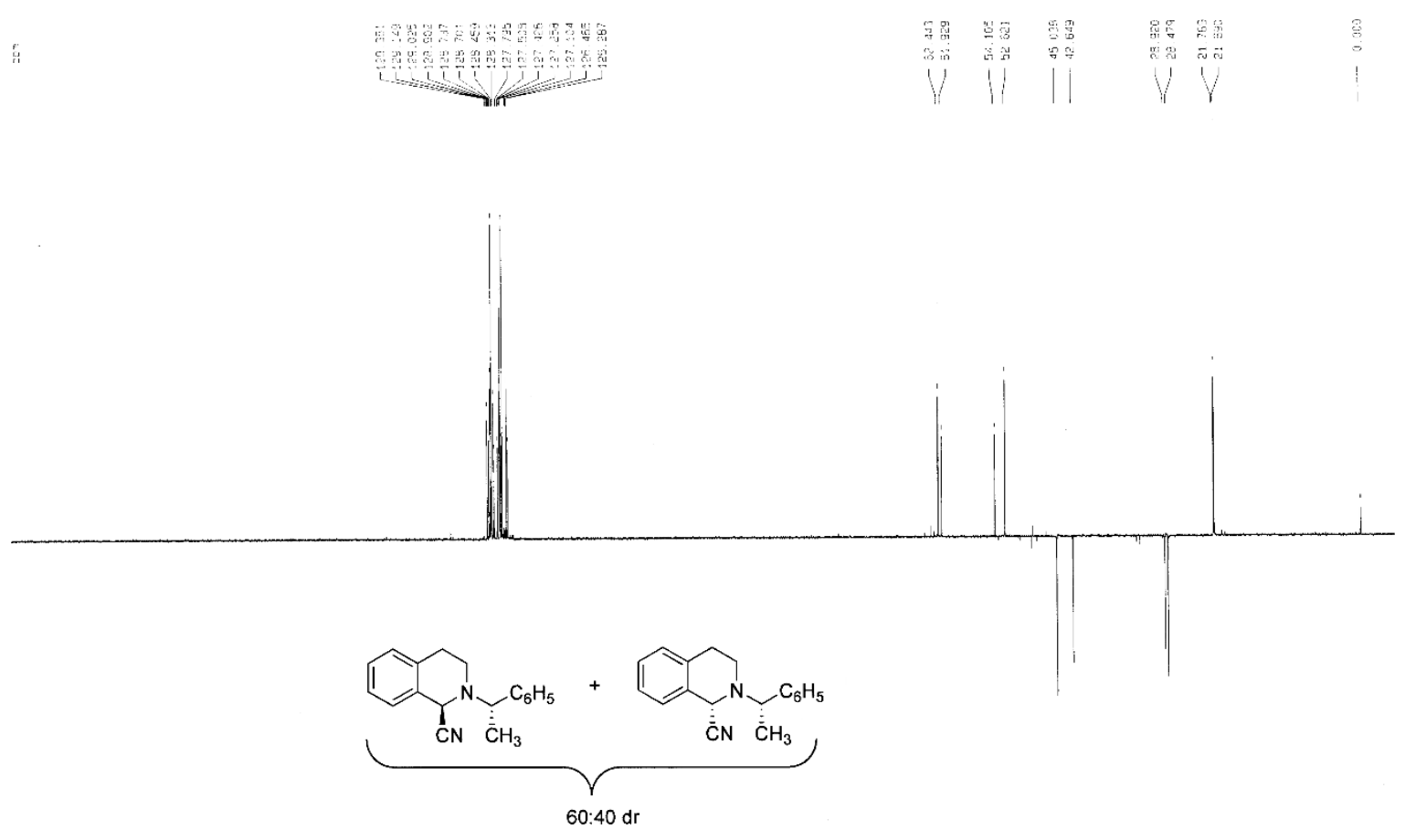

Figure S 22. DEPT C NMR spectrum ( $\left.\mathrm{CDCl}_{3}, 125 \mathrm{MHz}\right)$ of derivatives (+)-2a and (-)-2a. 


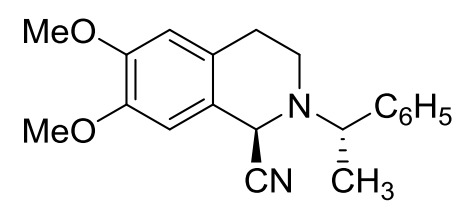

$(+)-2 b$

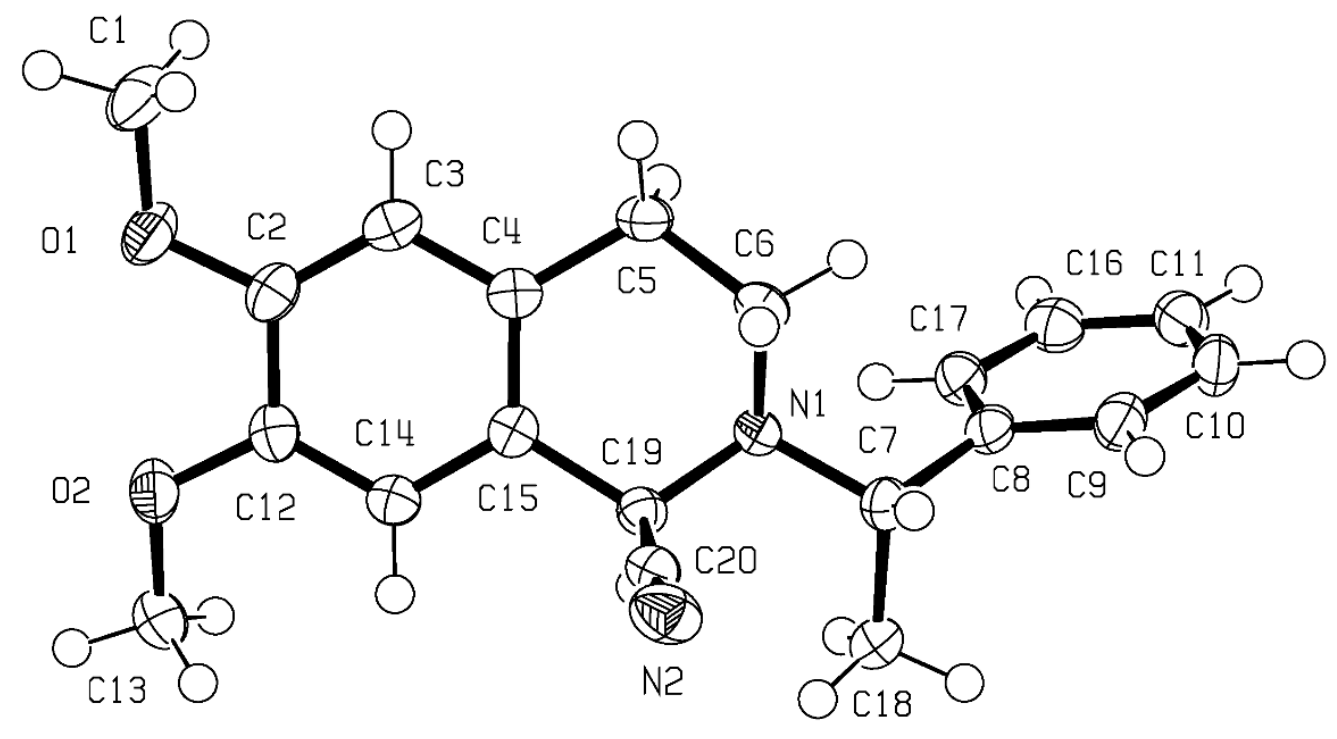

Figure S 23. ORTEP view of $\alpha$-amino nitrile (+)-2b. Ellipsoid plots are drawn with $70 \%$ of probability. 


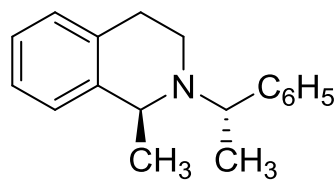

\section{$(-)-1 a$}

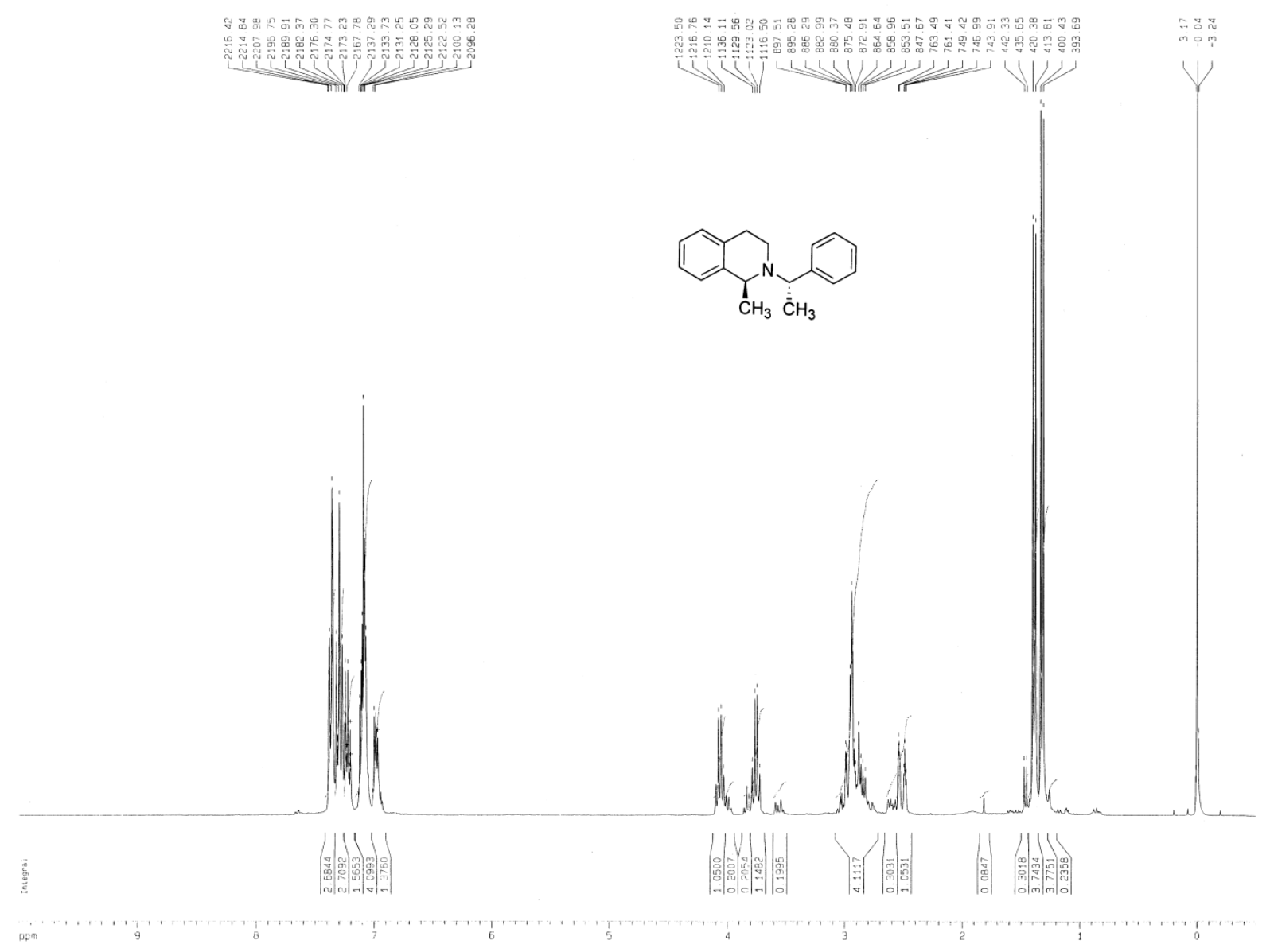

Figure S 24. ${ }^{1} \mathrm{H}$ NMR spectrum $\left(\mathrm{CDCl}_{3}, 300 \mathrm{MHz}\right)$ of derivative (-)-1a. 


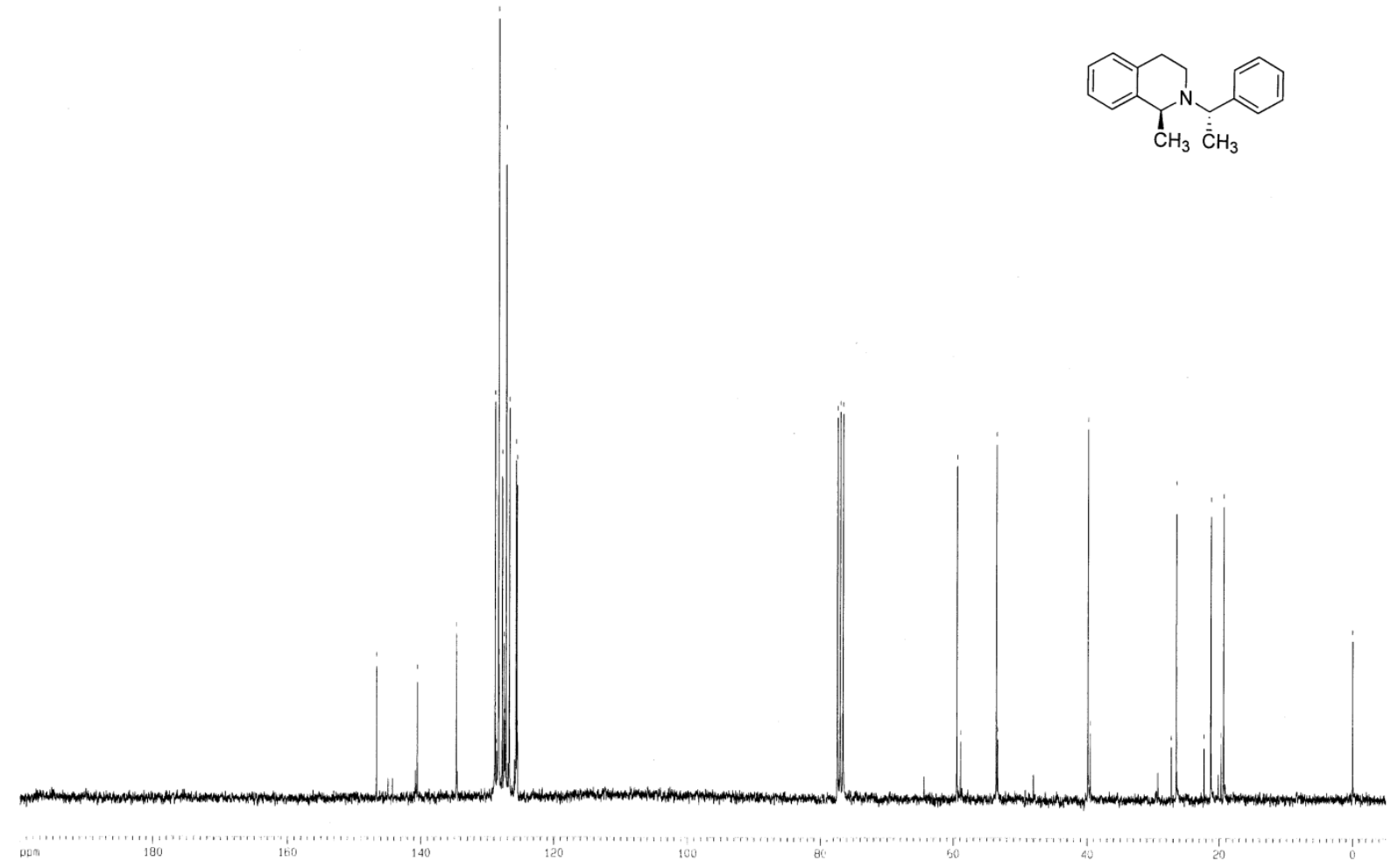

Figure S 25. ${ }^{13} \mathrm{C}$ NMR spectrum $\left(\mathrm{CDCl}_{3}, 75 \mathrm{MHz}\right)$ of derivative (-)-1a. 

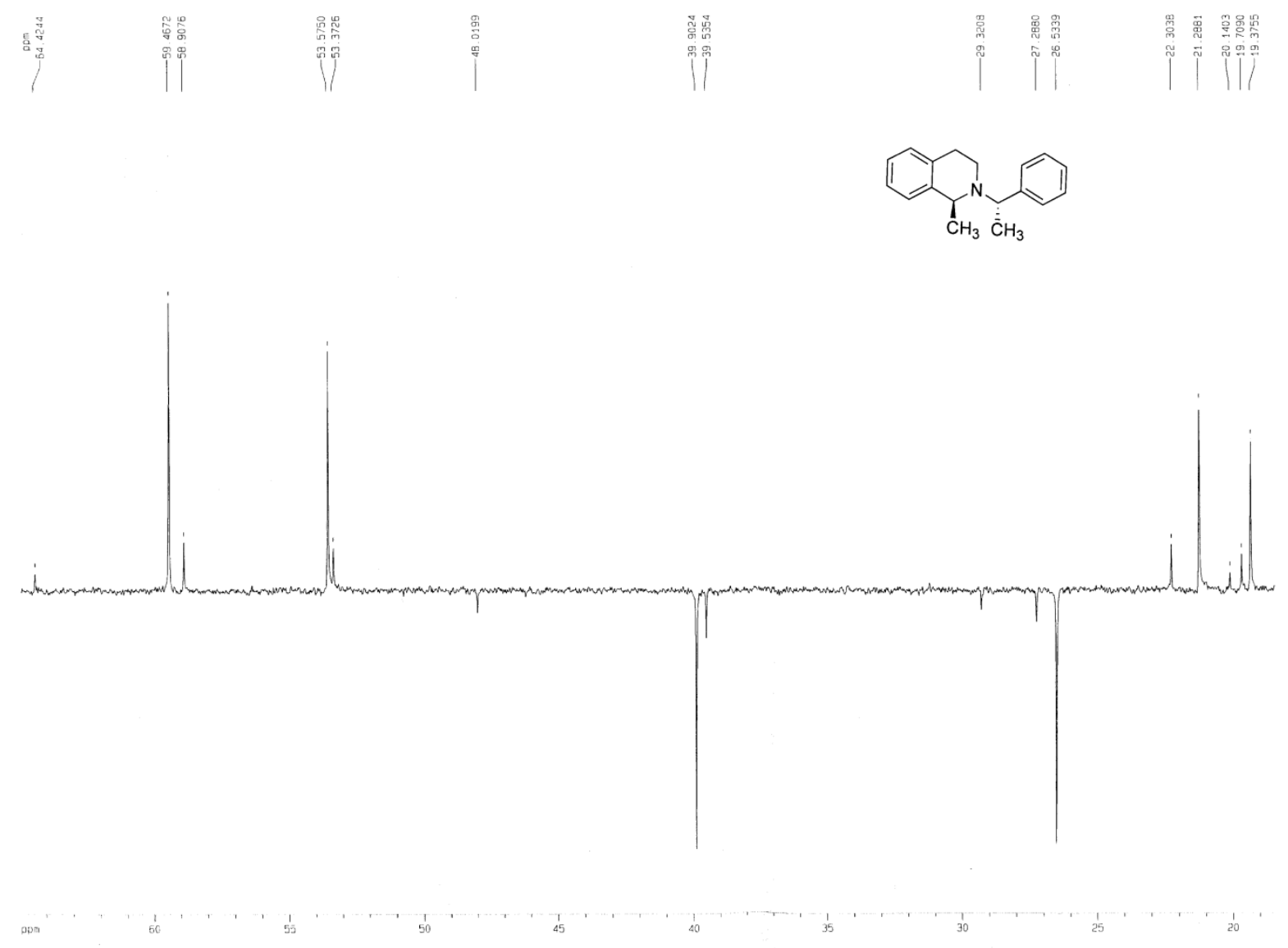

Figure $\mathbf{S}$ 26. DEPT C NMR spectrum $\left(\mathrm{CDCl}_{3}, 75 \mathrm{MHz}\right)$ of derivative (-)-1a. 


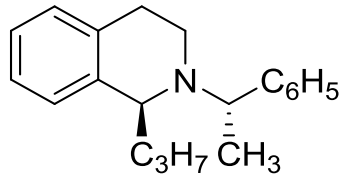

$(-)-1 b$

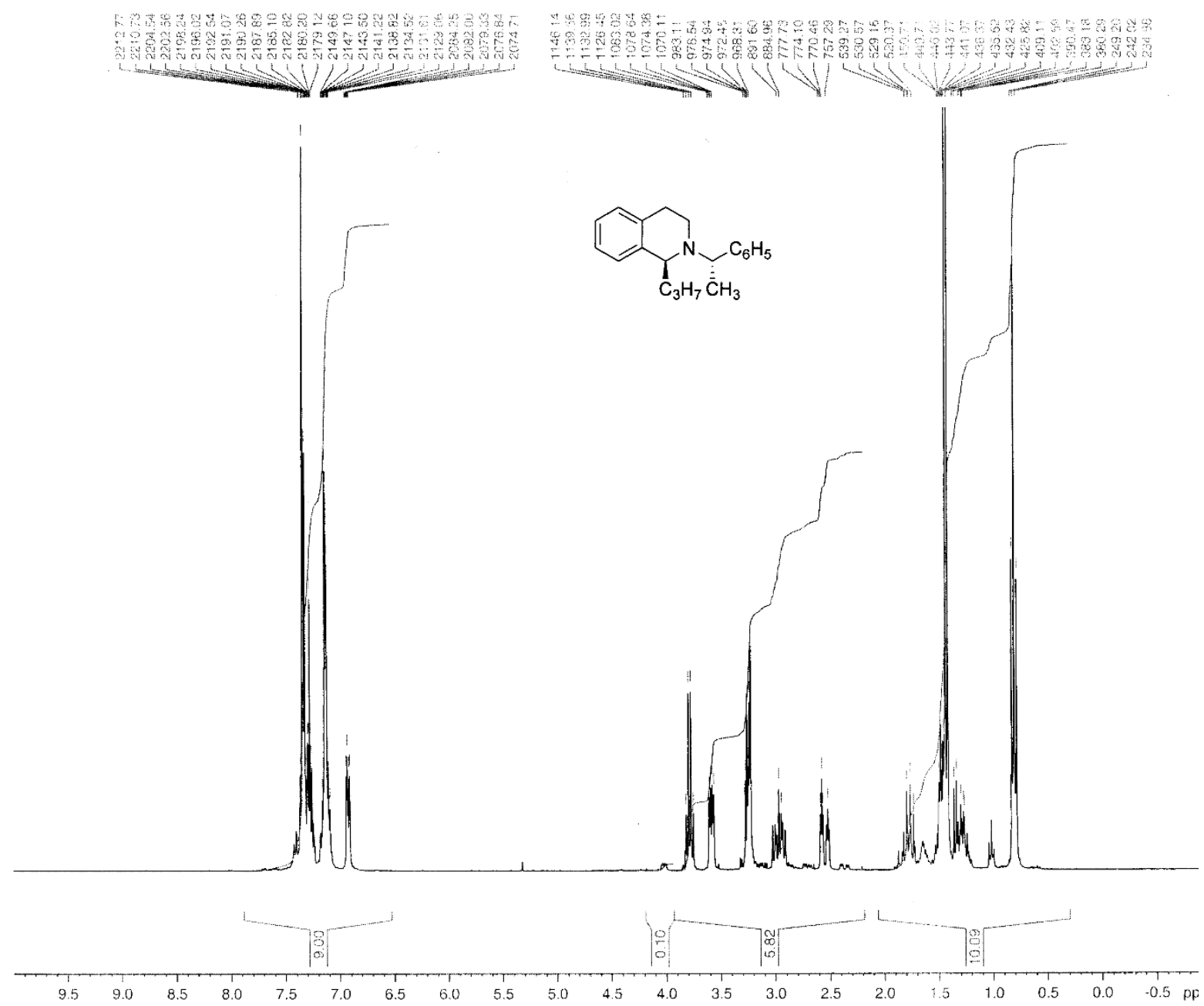

Figure $\mathbf{S}$ 27. ${ }^{1} \mathrm{H}$ NMR spectrum $\left(\mathrm{CDCl}_{3}, 300 \mathrm{MHz}\right)$ of derivative $(-)-\mathbf{1} \mathbf{b}$. 


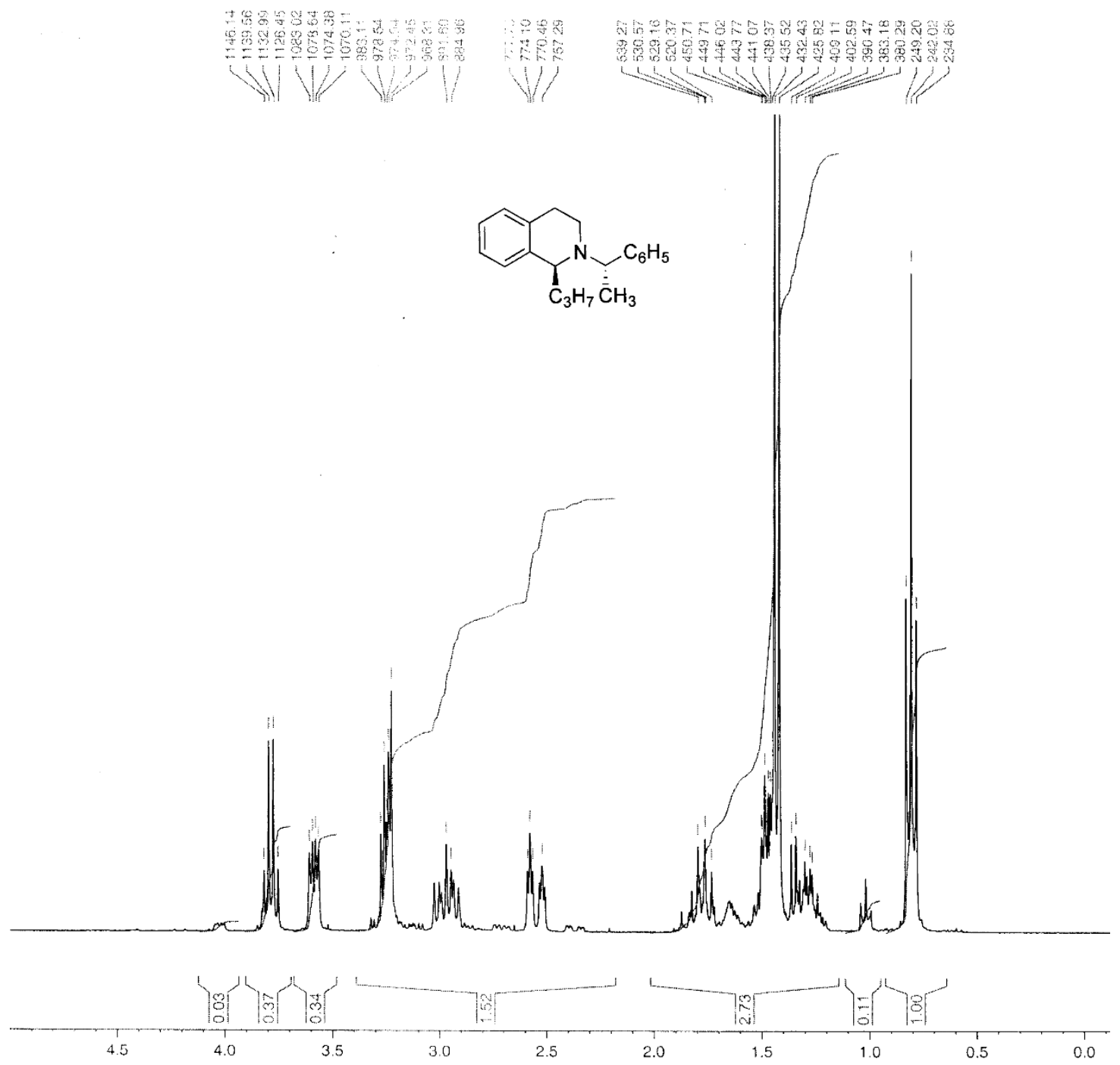

Figure $\mathbf{S}$ 28. ${ }^{1} \mathrm{H}$ NMR spectrum $\left(\mathrm{CDCl}_{3}, 300 \mathrm{MHz}\right)$ of derivative $(-)-\mathbf{1} \mathbf{b}$. 
高:

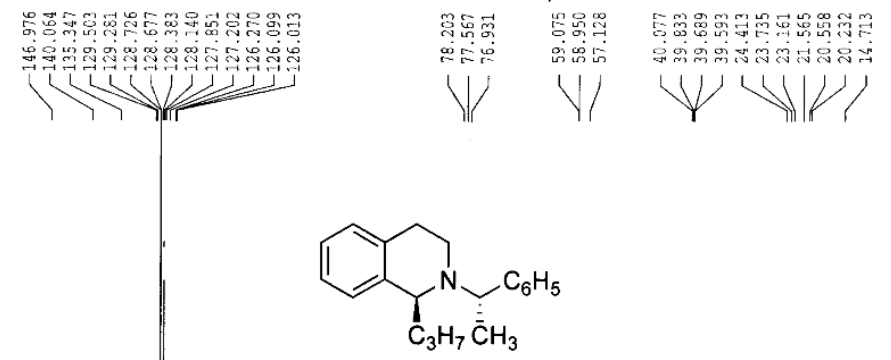

song

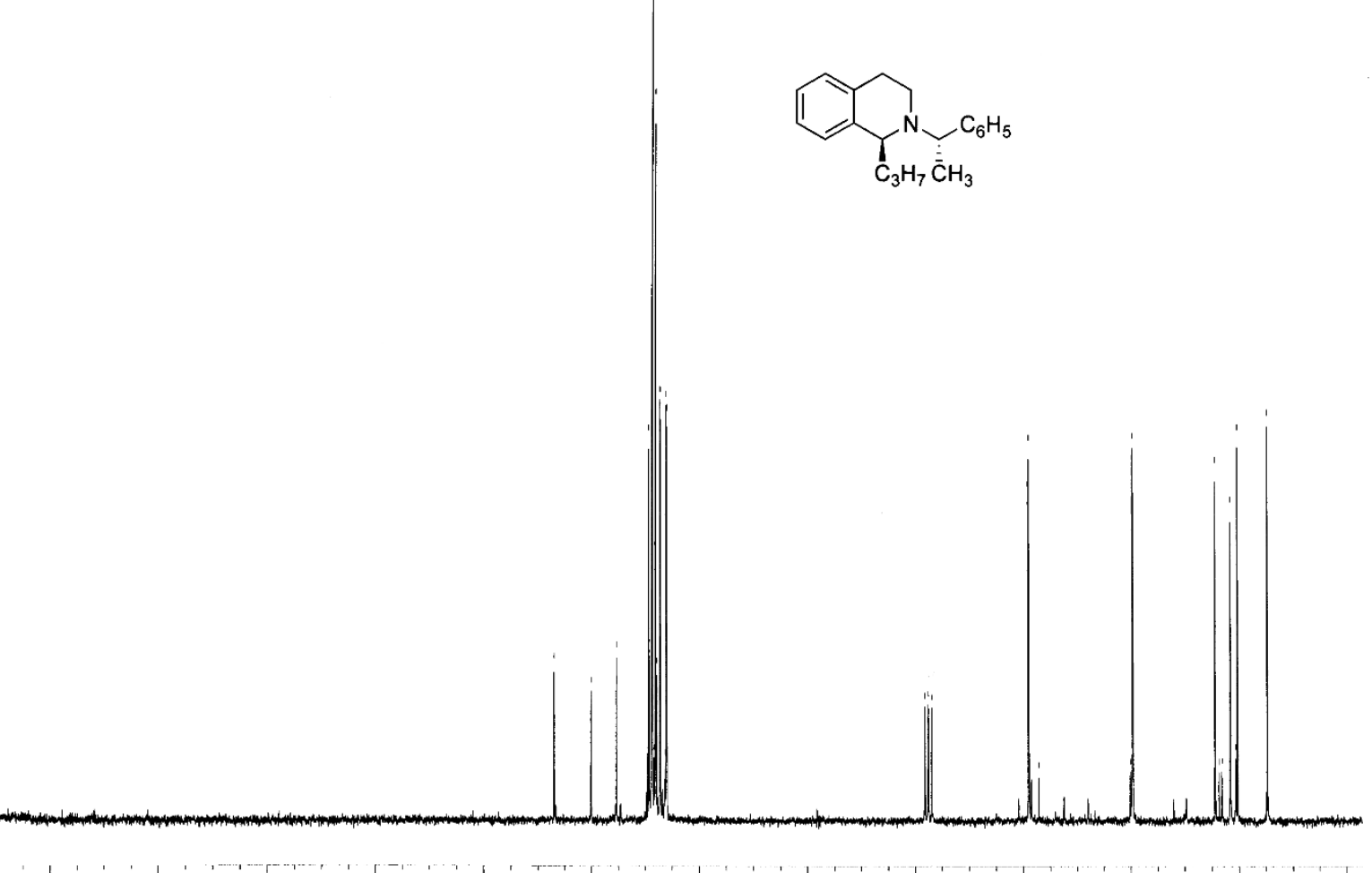

gemin 240

160 ' 140

120

100

Figure S 29. ${ }^{13} \mathrm{C}$ NMR spectrum $\left(\mathrm{CDCl}_{3}, 75 \mathrm{MHz}\right)$ of derivative (-)-1b. 


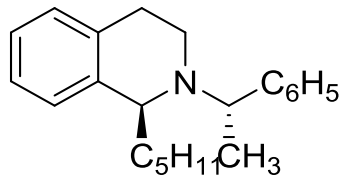

(-)-1c

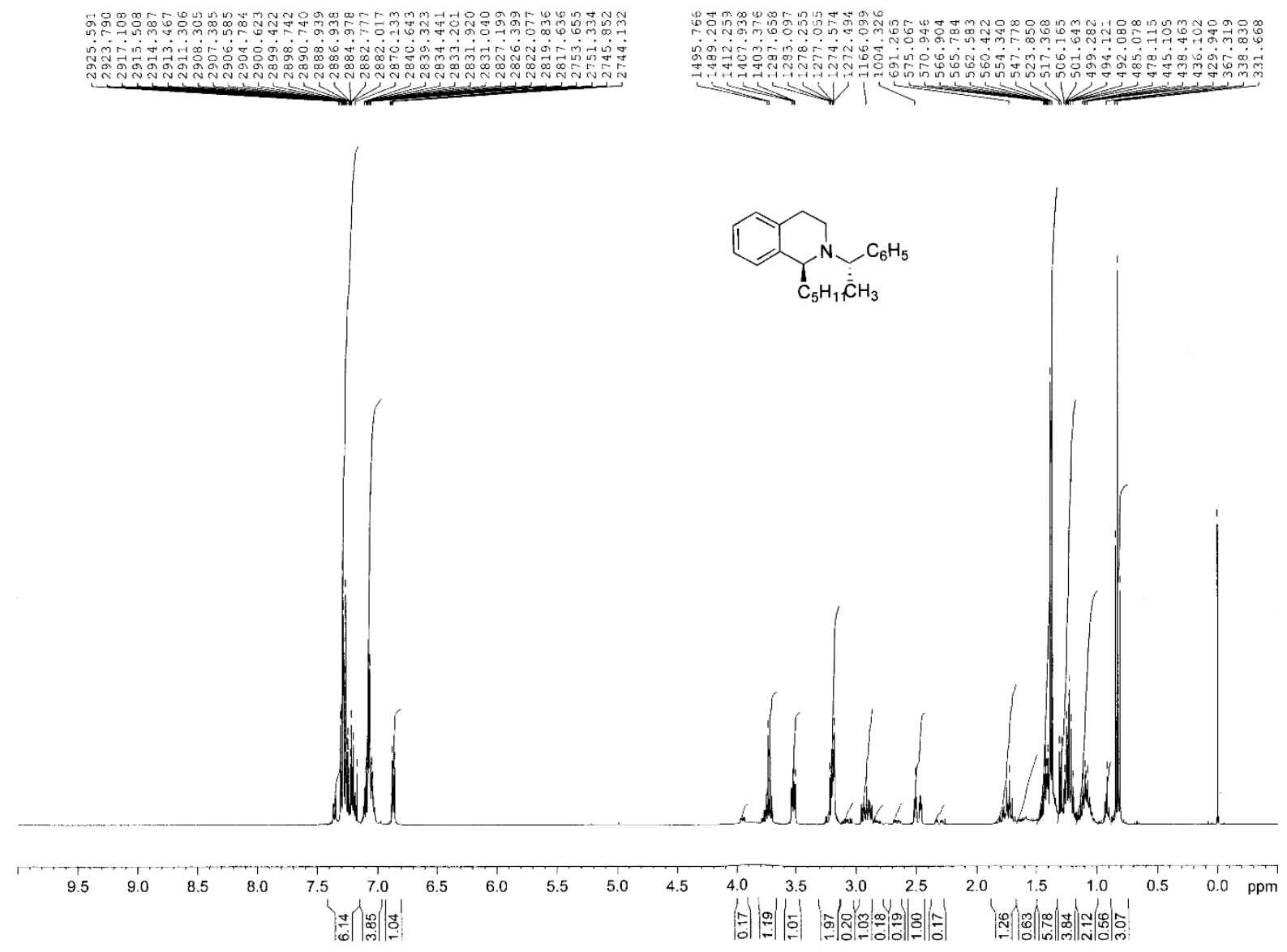

Figure S 30. ${ }^{1} \mathrm{H}$ NMR spectrum $\left(\mathrm{CDCl}_{3}, 400 \mathrm{MHz}\right)$ of derivative (-)-1c. 


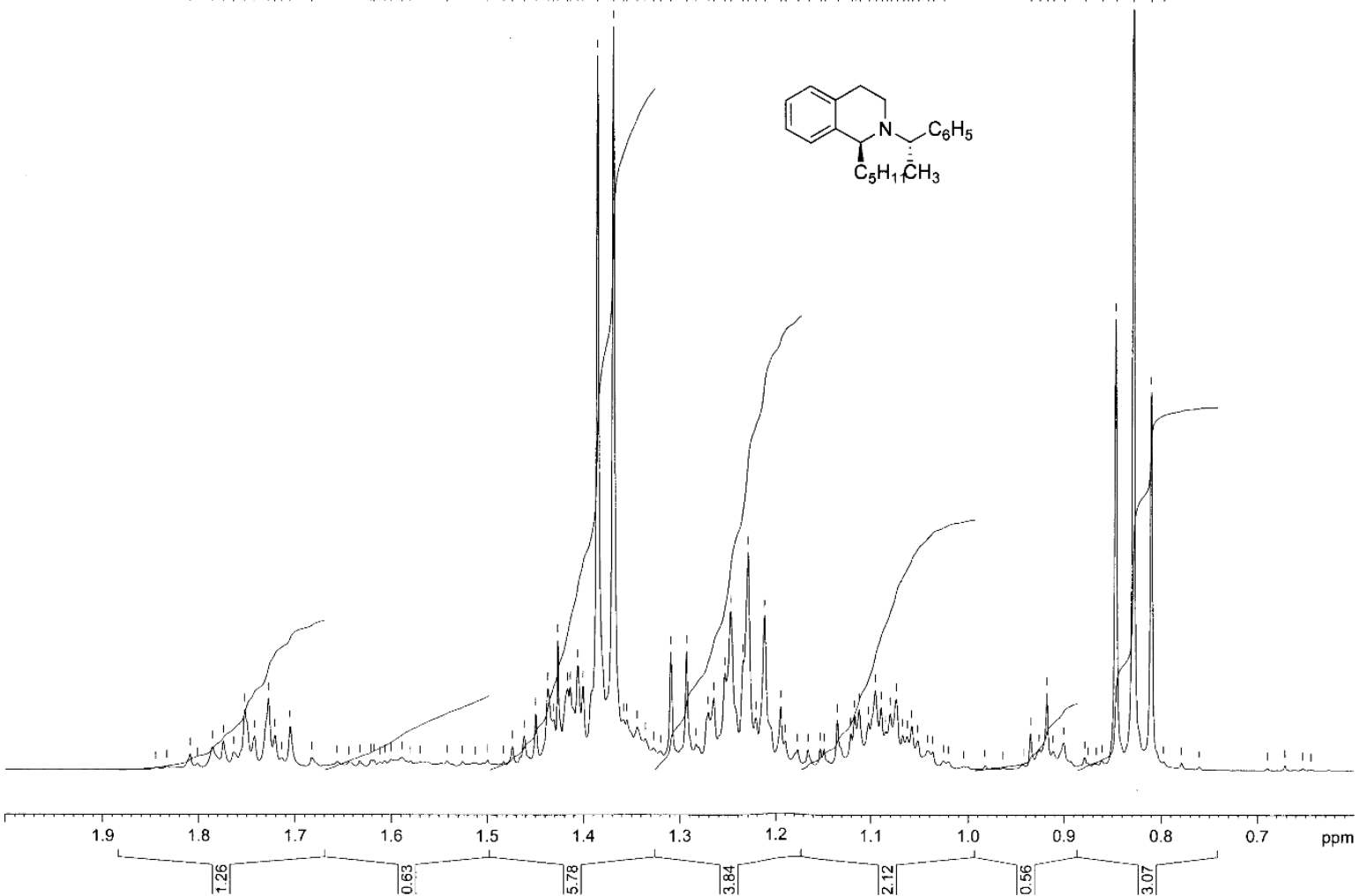

Figure S 31. ${ }^{1} \mathrm{H}$ NMR spectrum $\left(\mathrm{CDCl}_{3}, 400 \mathrm{MHz}\right)$ of derivative (-)-1c. 


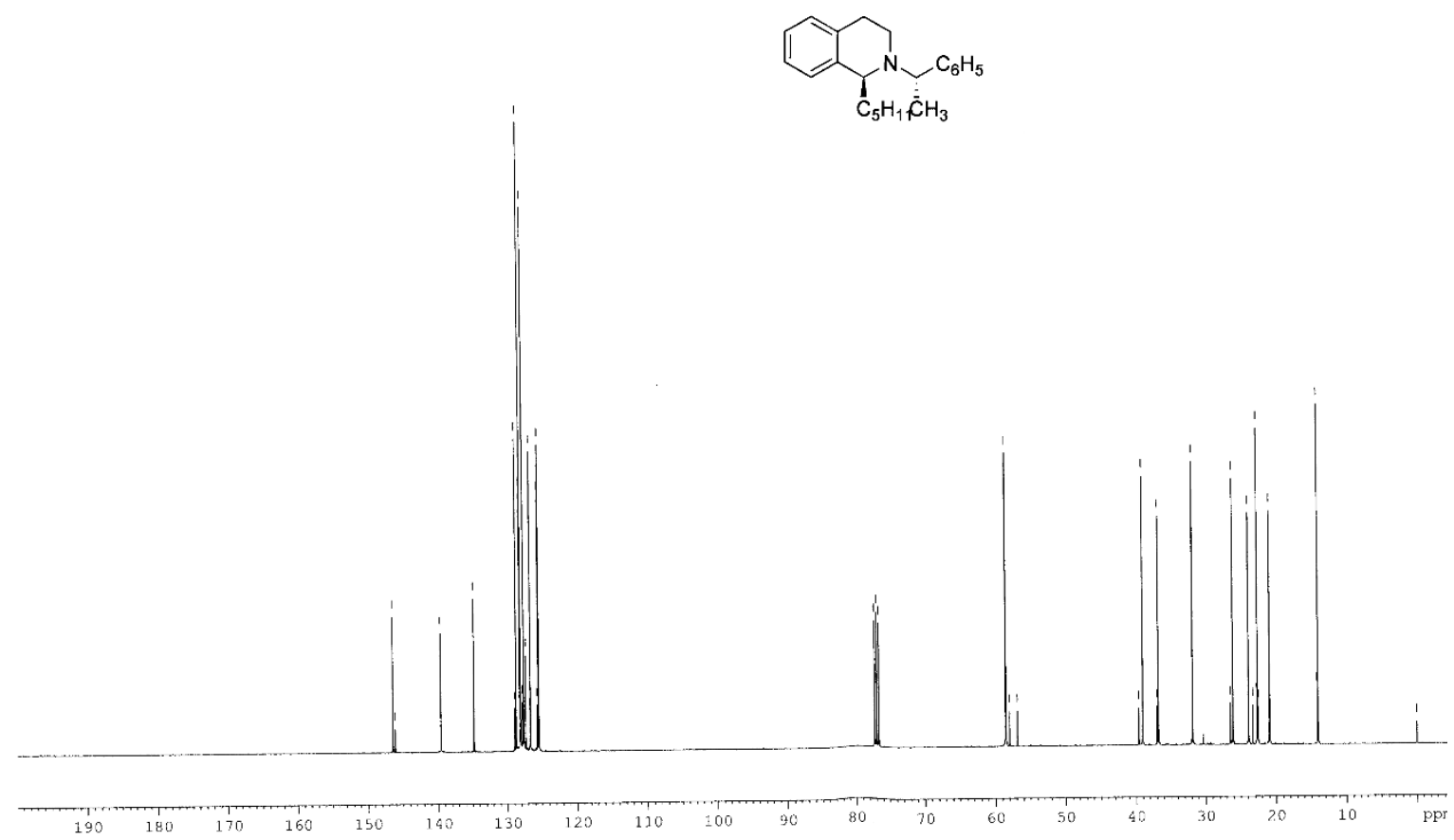

Figure S 32. ${ }^{13} \mathrm{C}$ NMR spectrum $\left(\mathrm{CDCl}_{3}, 100 \mathrm{MHz}\right)$ of derivative $(-)-\mathbf{1 c}$. 


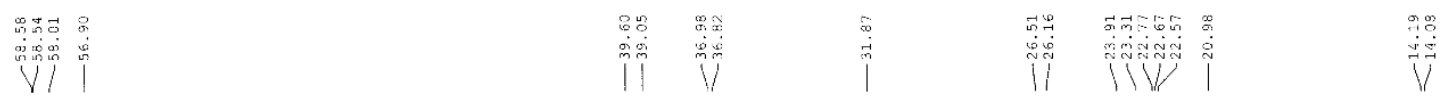

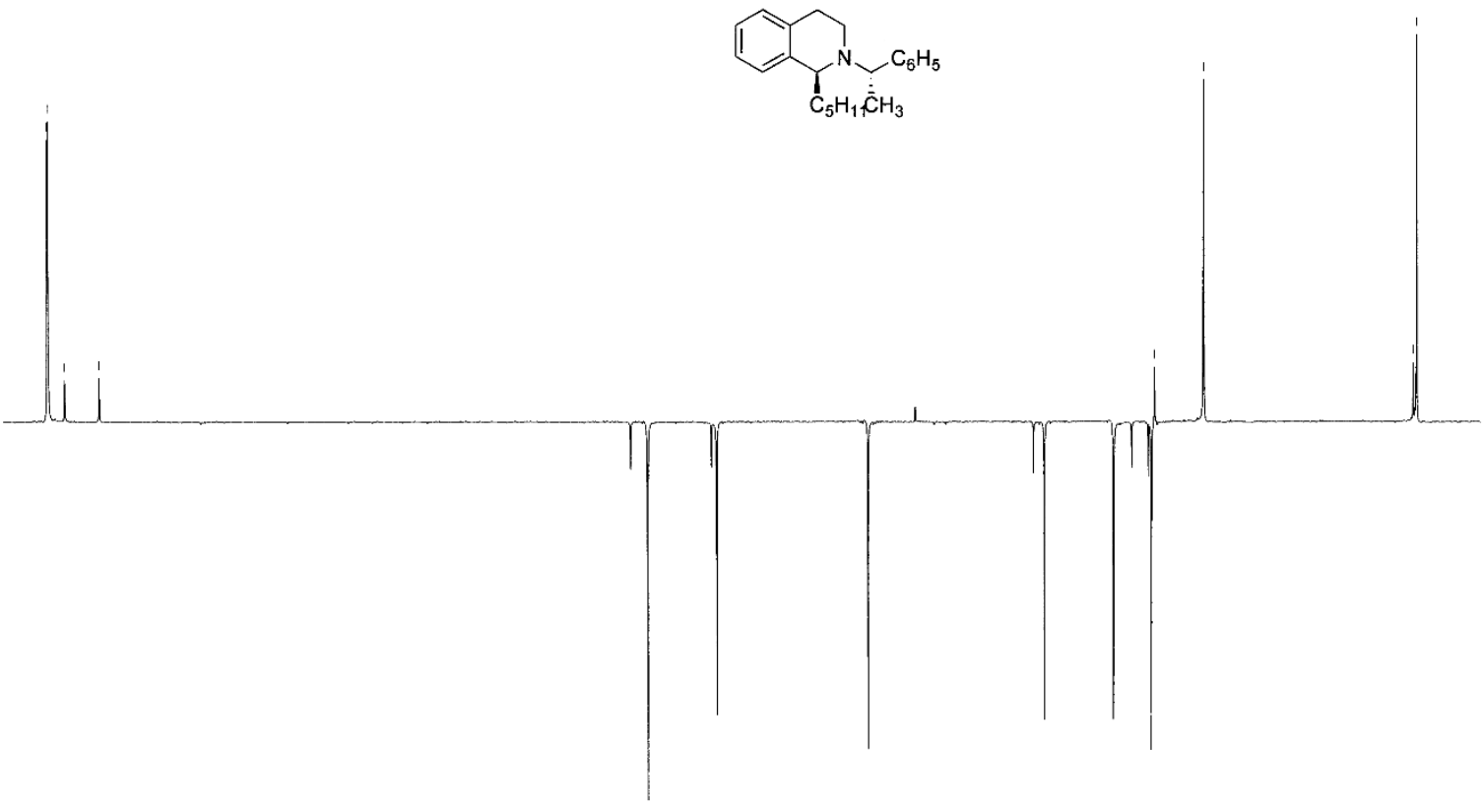

Figure S 33. DEPT C NMR spectrum $\left(\mathrm{CDCl}_{3}, 100 \mathrm{MHz}\right)$ of derivative (-)-1c. 


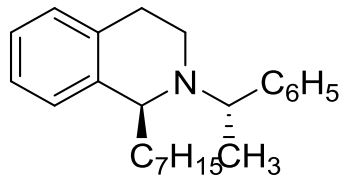

(-)-1d

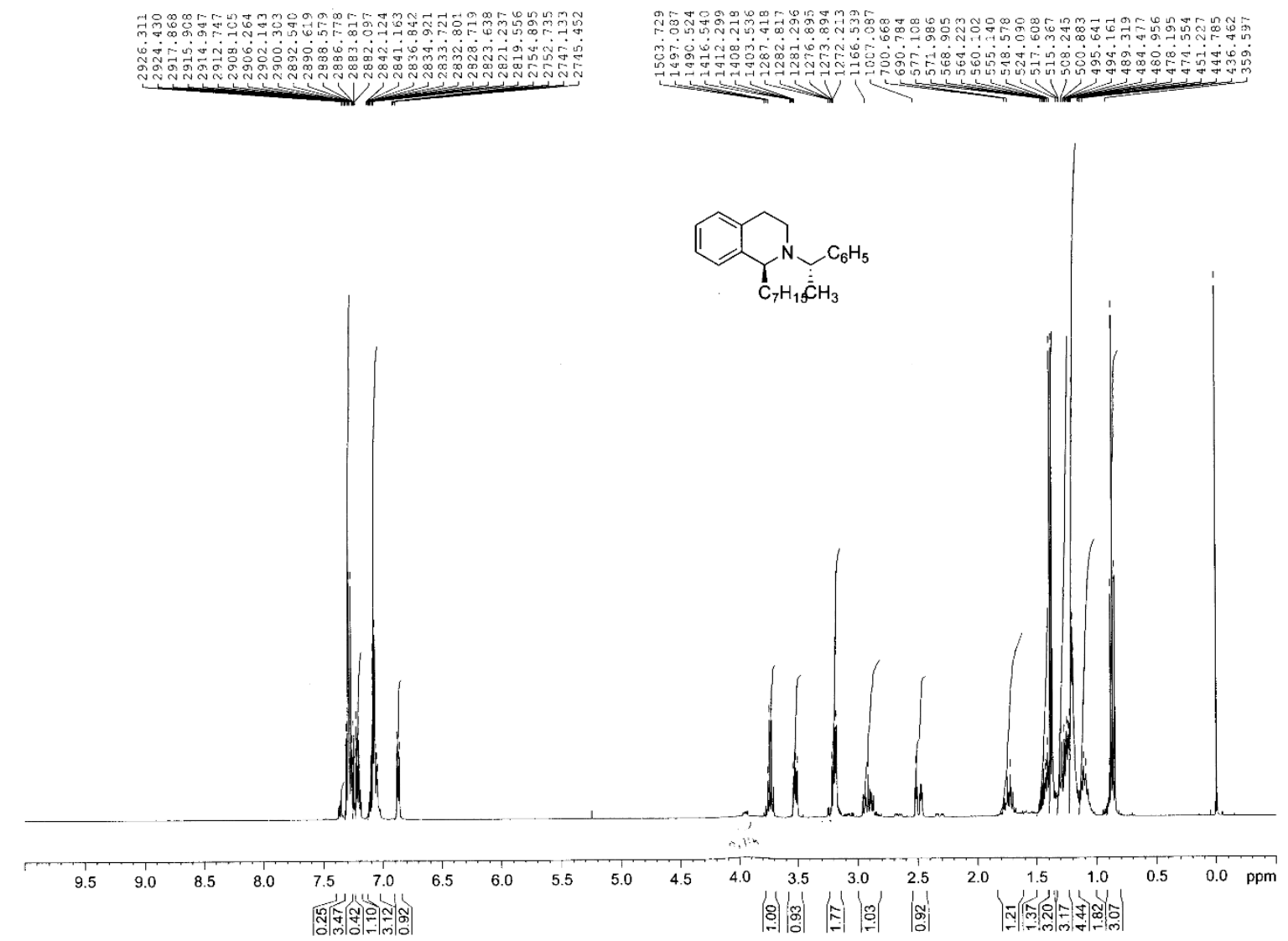

Figure S 34. ${ }^{1} \mathrm{H}$ NMR spectrum $\left(\mathrm{CDCl}_{3}, 400 \mathrm{MHz}\right)$ of derivative (-)-1d. 


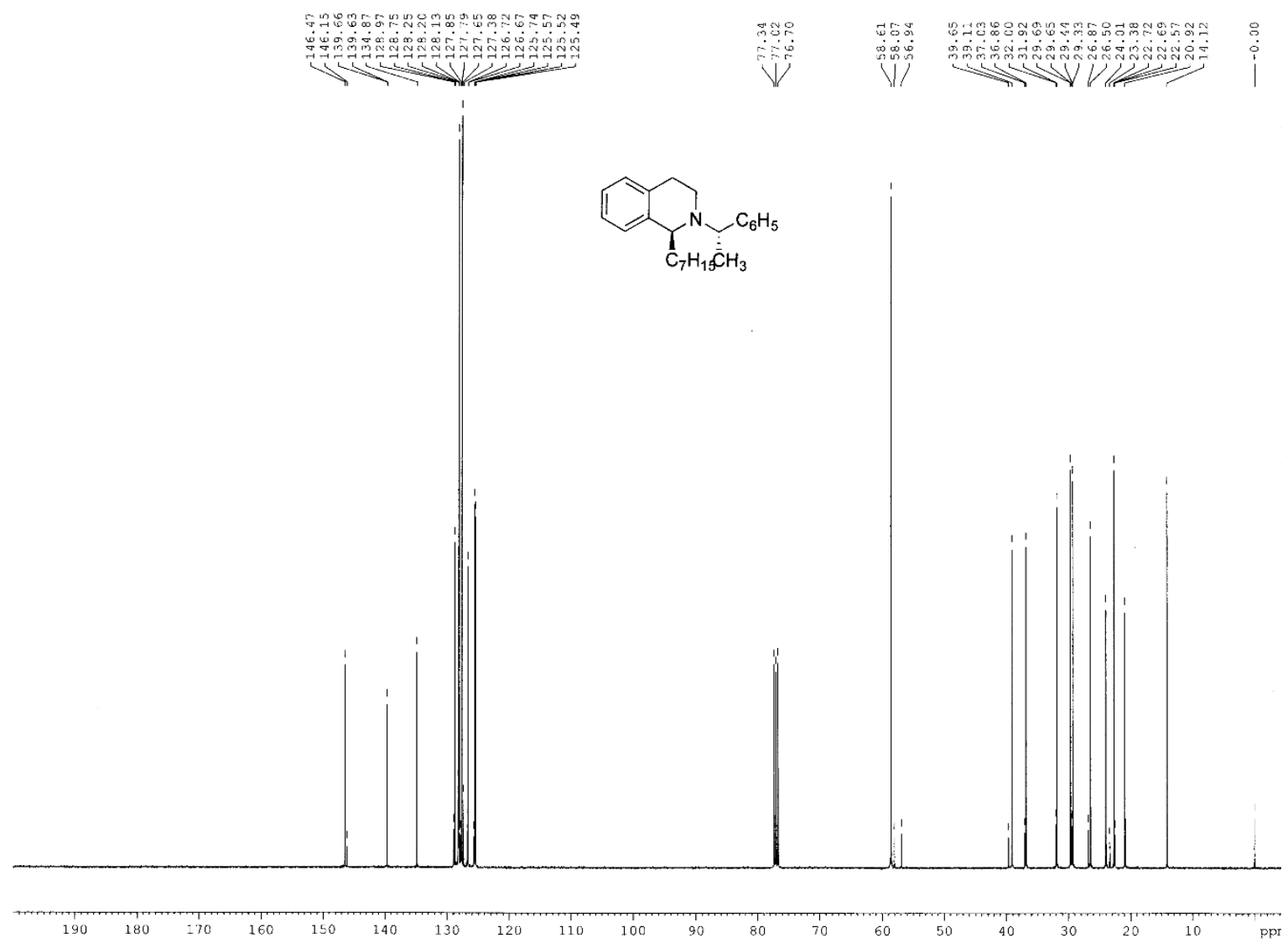

Figure S $35 .{ }^{13} \mathrm{C}$ NMR spectrum $\left(\mathrm{CDCl}_{3}, 100 \mathrm{MHz}\right)$ of derivative (-)-1d. 


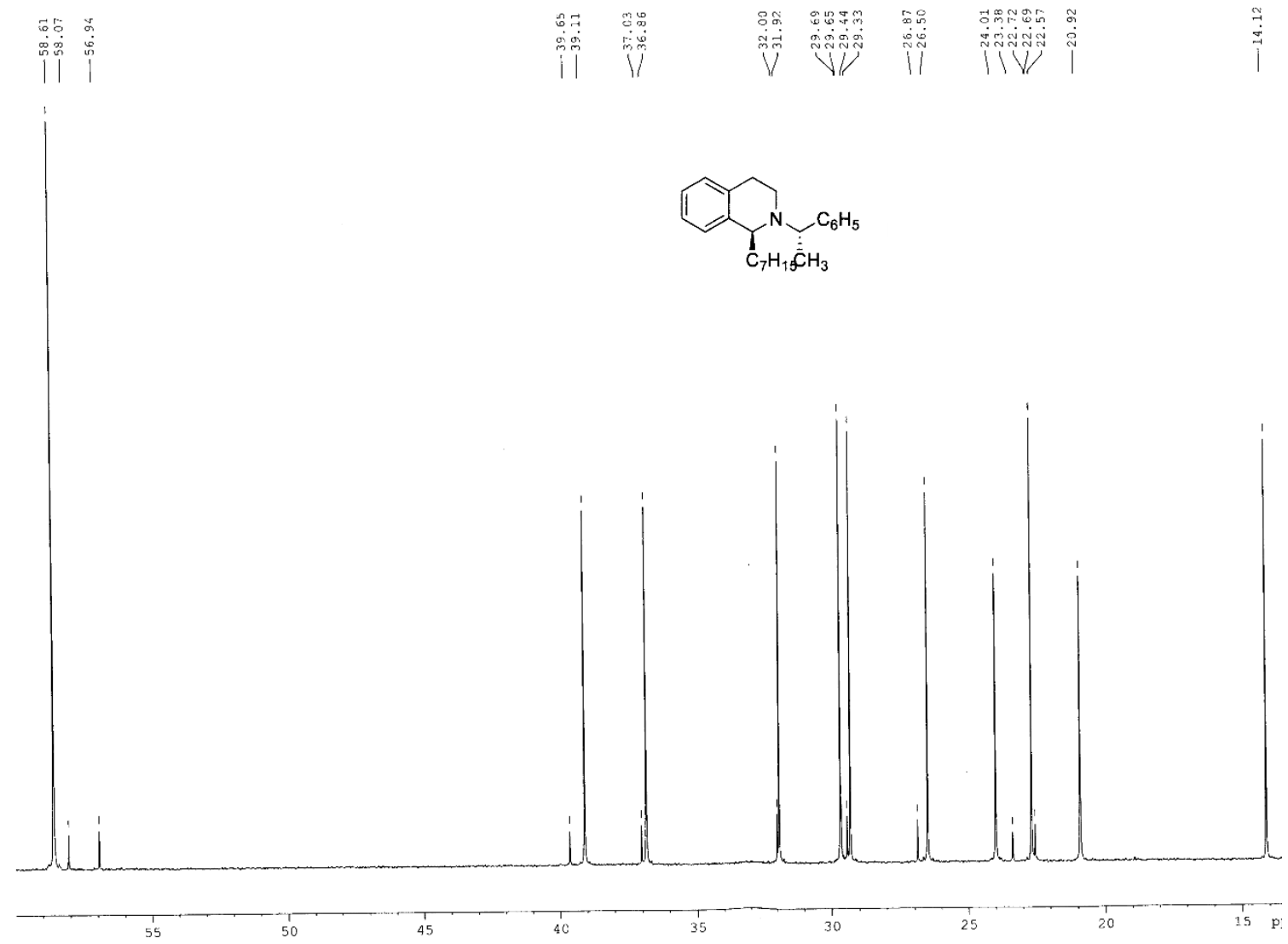

Figure S 36. ${ }^{13} \mathrm{C}$ NMR spectrum $\left(\mathrm{CDCl}_{3}, 100 \mathrm{MHz}\right)$ of derivative (-)-1d. 


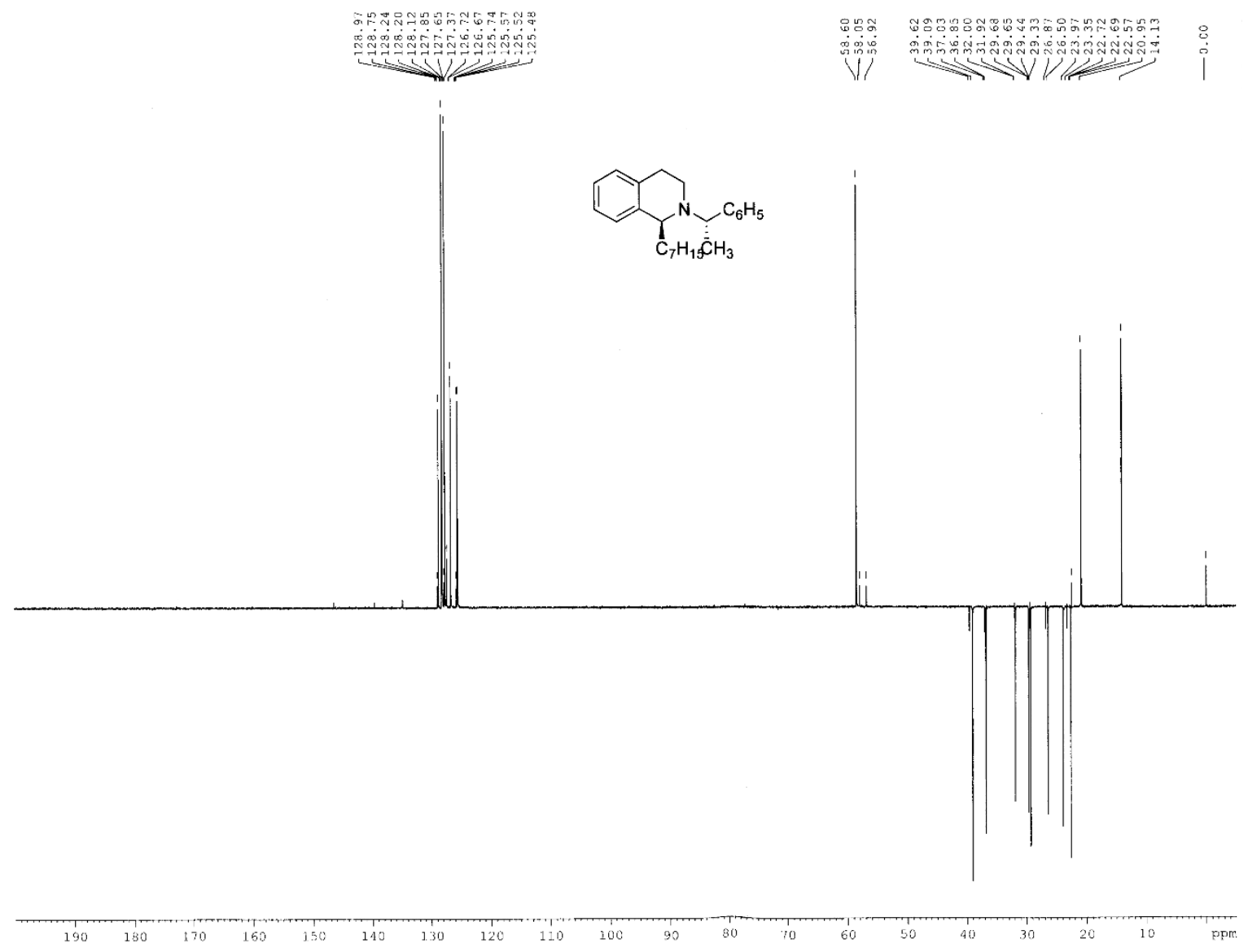

Figure S 37. DEPT C NMR spectrum $\left(\mathrm{CDCl}_{3}, 100 \mathrm{MHz}\right)$ of derivative (-)-1d. 


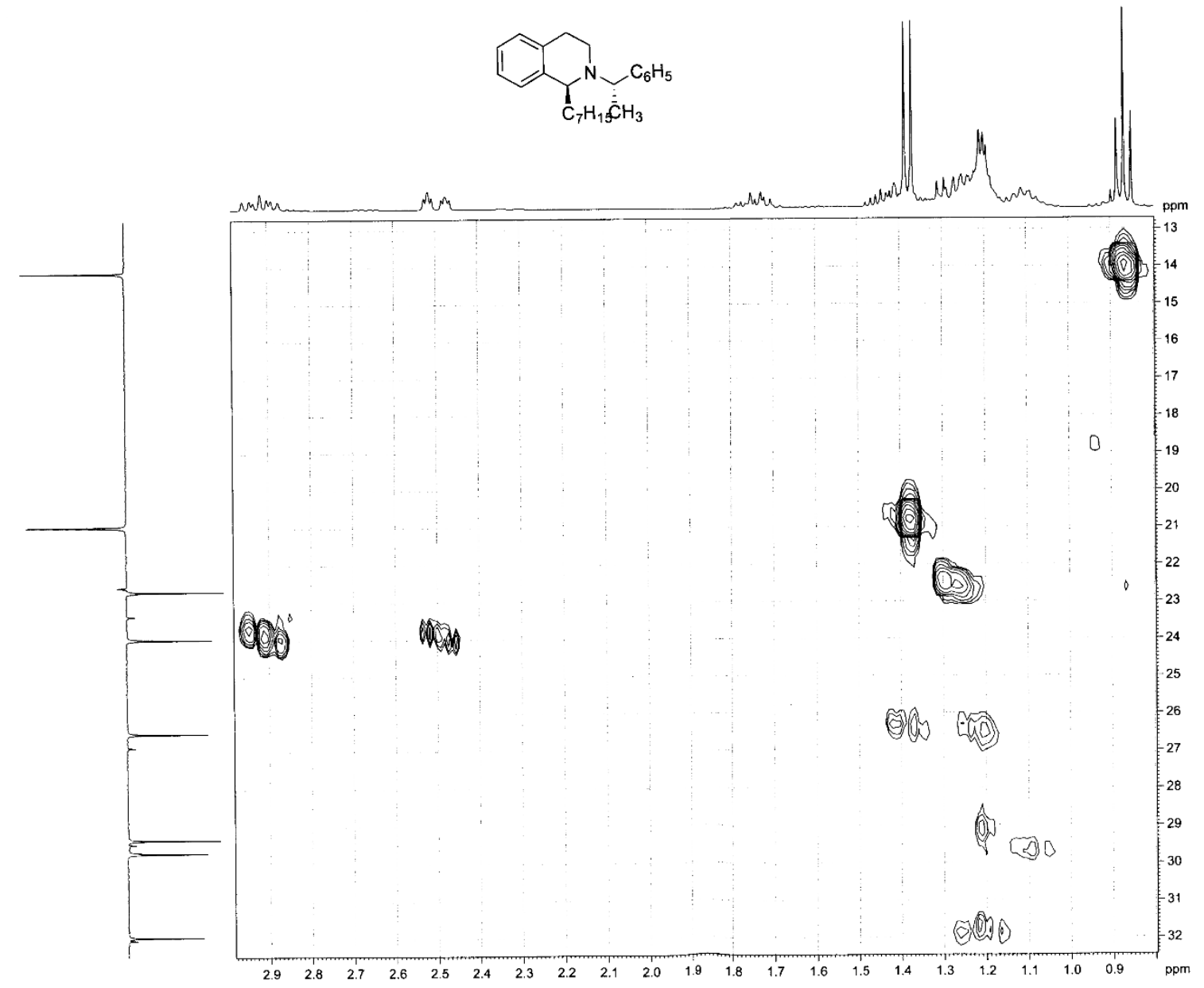

Figure S 38. HMQC spectrum $\left(\mathrm{CDCl}_{3}\right)$ of derivative (-)-1d. 


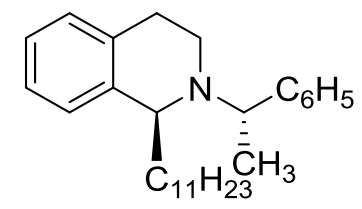

$$
(-)-1 e
$$

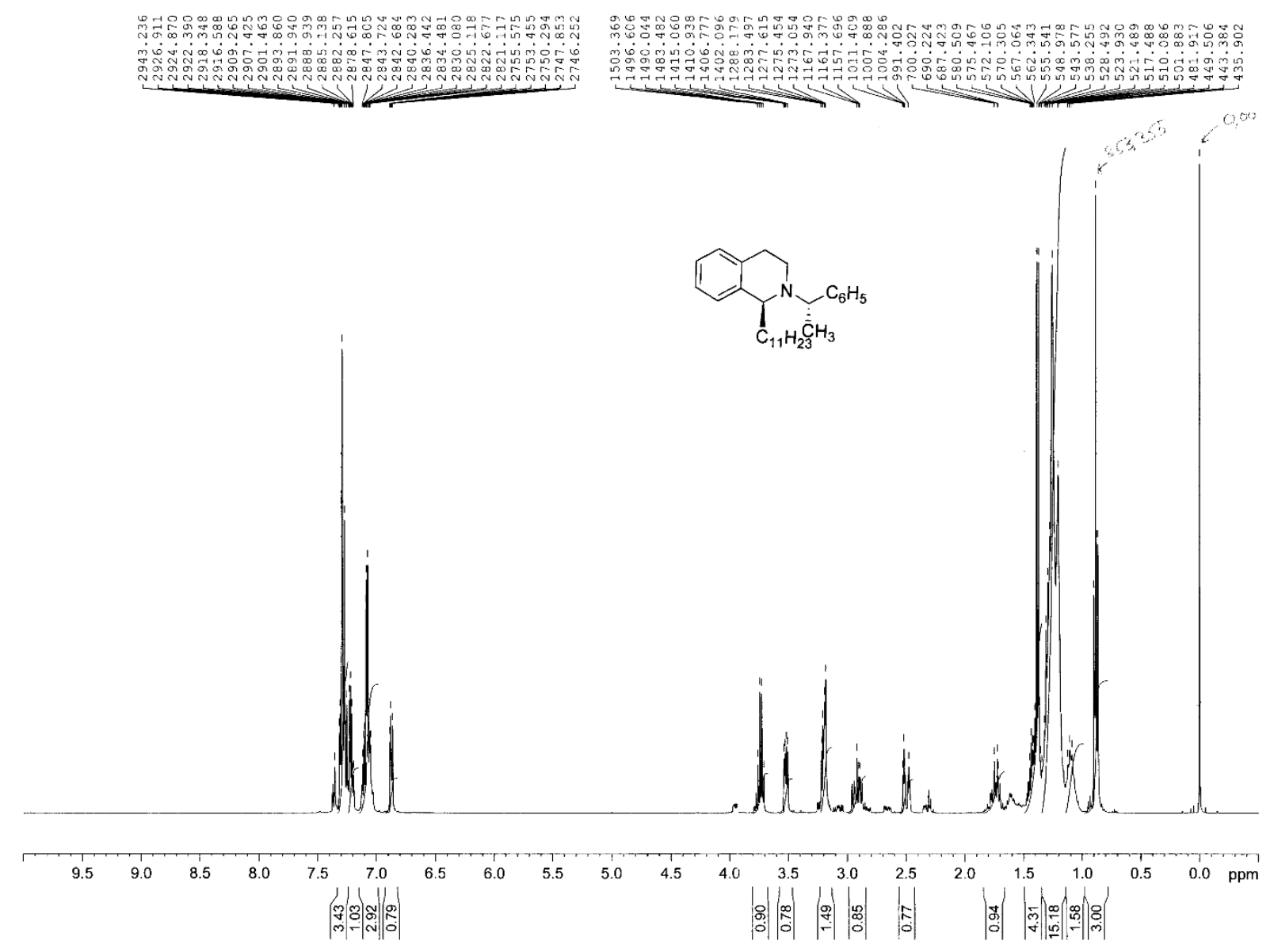

Figure S 39. ${ }^{1} \mathrm{H}$ NMR spectrum $\left(\mathrm{CDCl}_{3}, 400 \mathrm{MHz}\right)$ of derivative (-)-1e. 


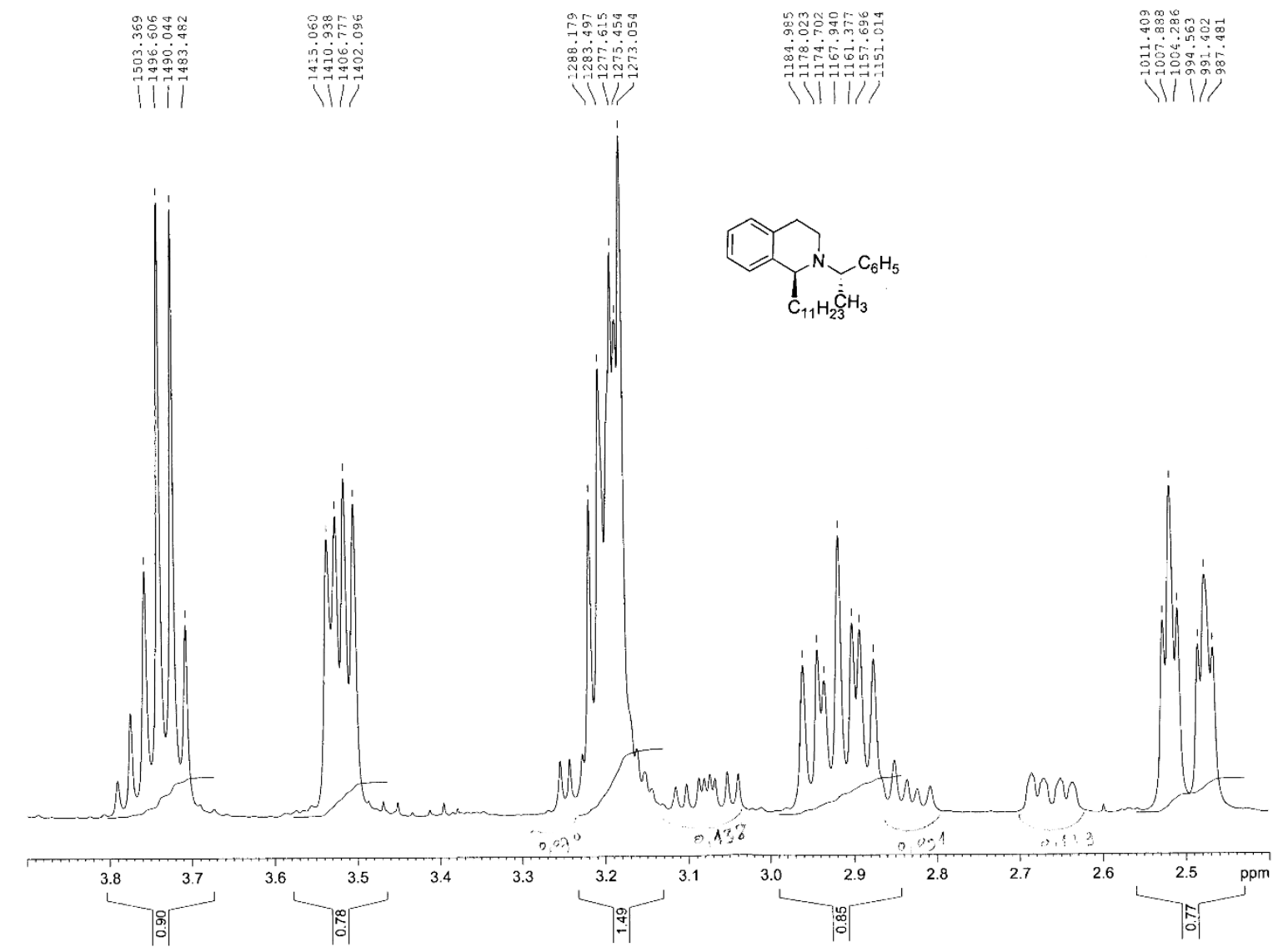

Figure S 40. ${ }^{1} \mathrm{H} \mathrm{NMR}$ spectrum $\left(\mathrm{CDCl}_{3}, 400 \mathrm{MHz}\right)$ of derivative (-)-1e. 
S43

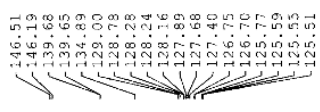

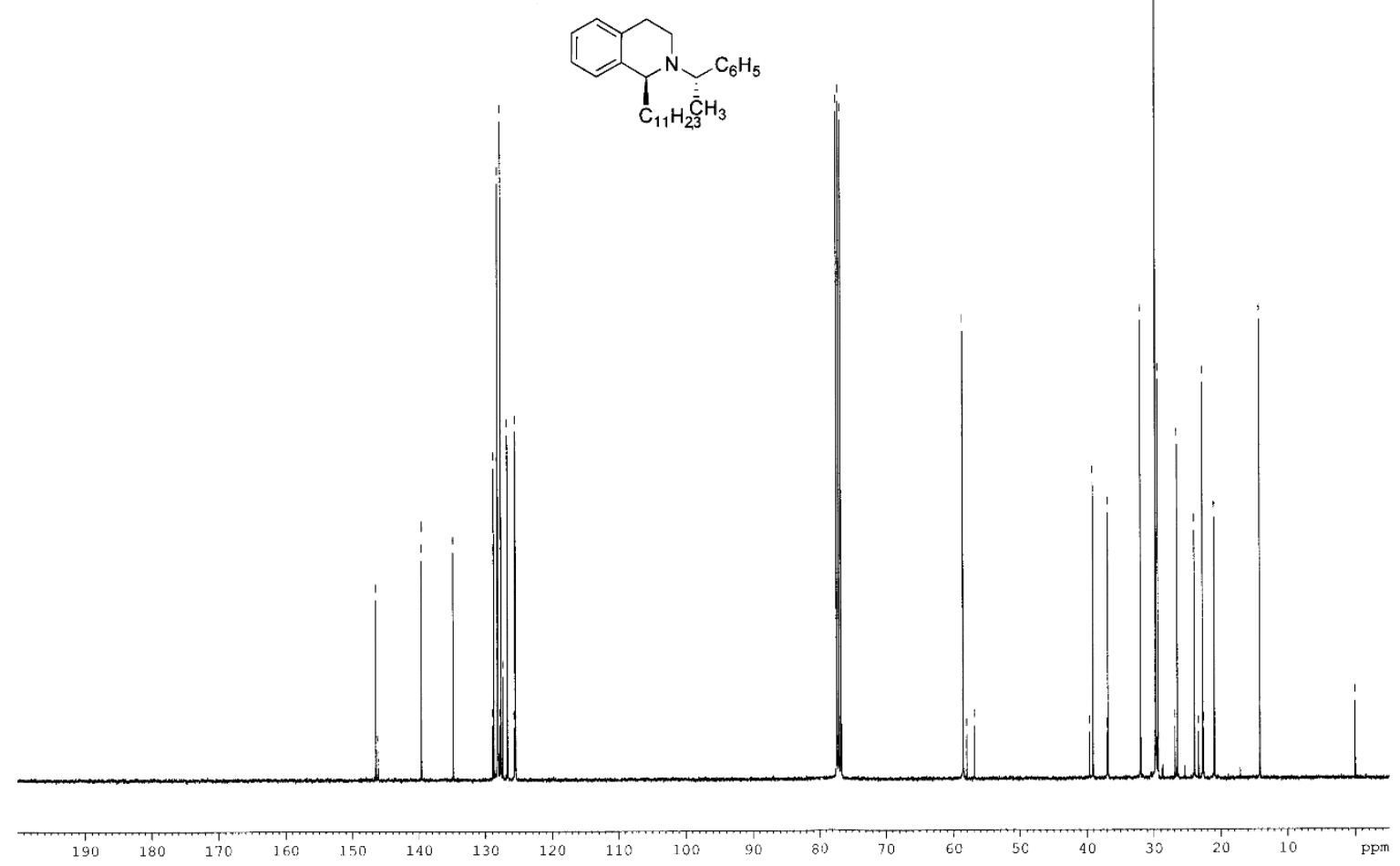

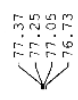

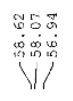

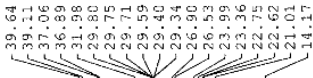

Figure S 41. ${ }^{13} \mathrm{C}$ NMR spectrum $\left(\mathrm{CDCl}_{3}, 100 \mathrm{MHz}\right)$ of derivative (-)-ie. 
S44
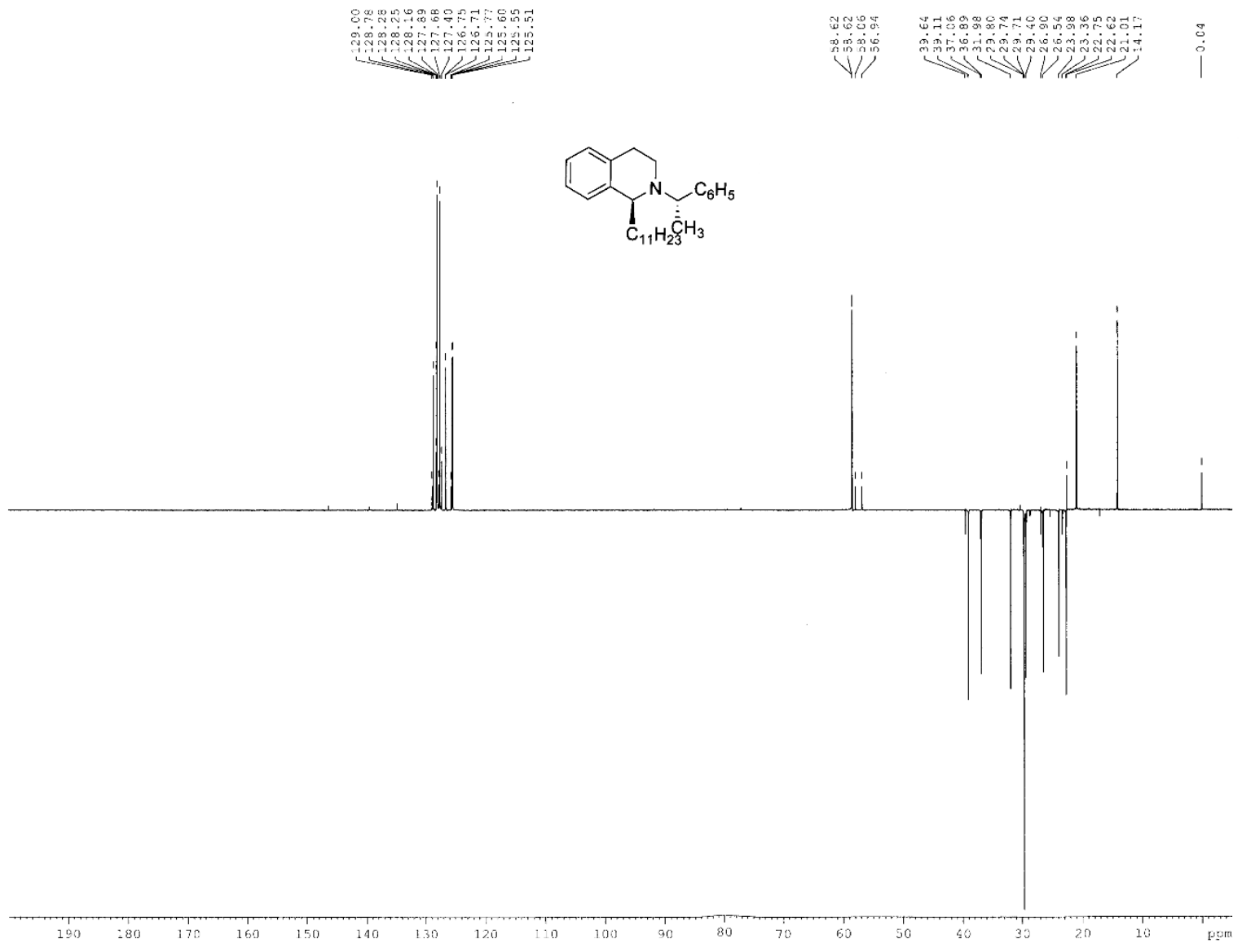

Figure $\mathbf{S}$ 42. DEPT C NMR spectrum $\left(\mathrm{CDCl}_{3}, 100 \mathrm{MHz}\right)$ of derivative (-)-ie.

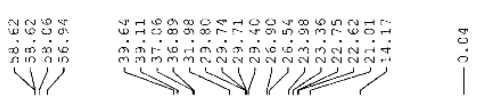




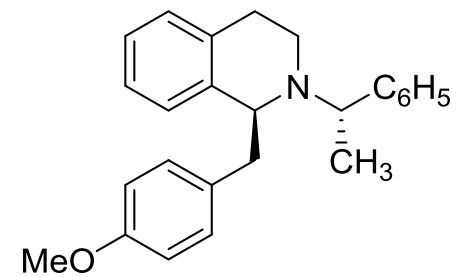

$(+)-1 f$

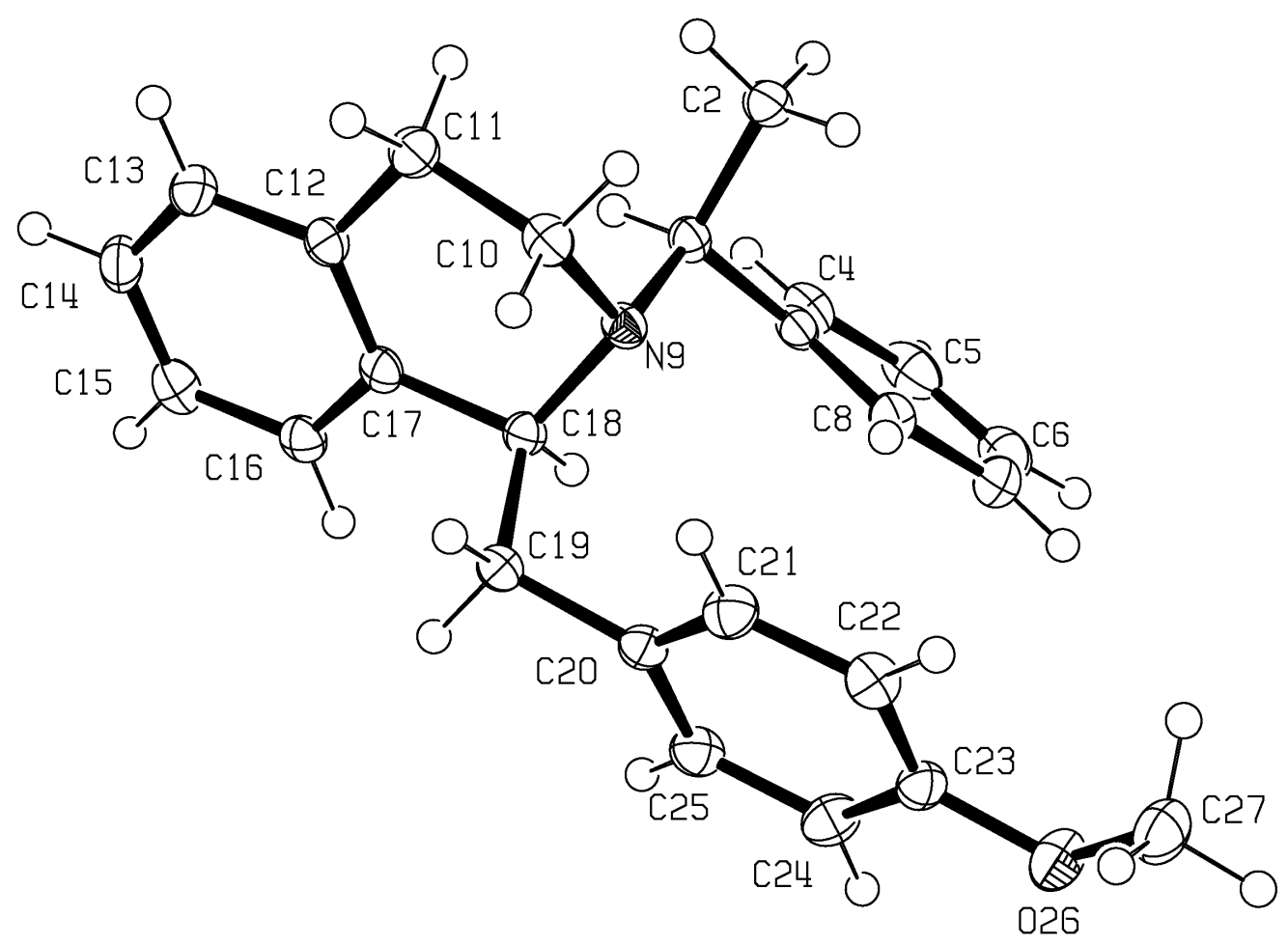

Figure S 43. ORTEP view of derivative (+)-1f. Ellipsoid plots are drawn with $70 \%$ of probability. 


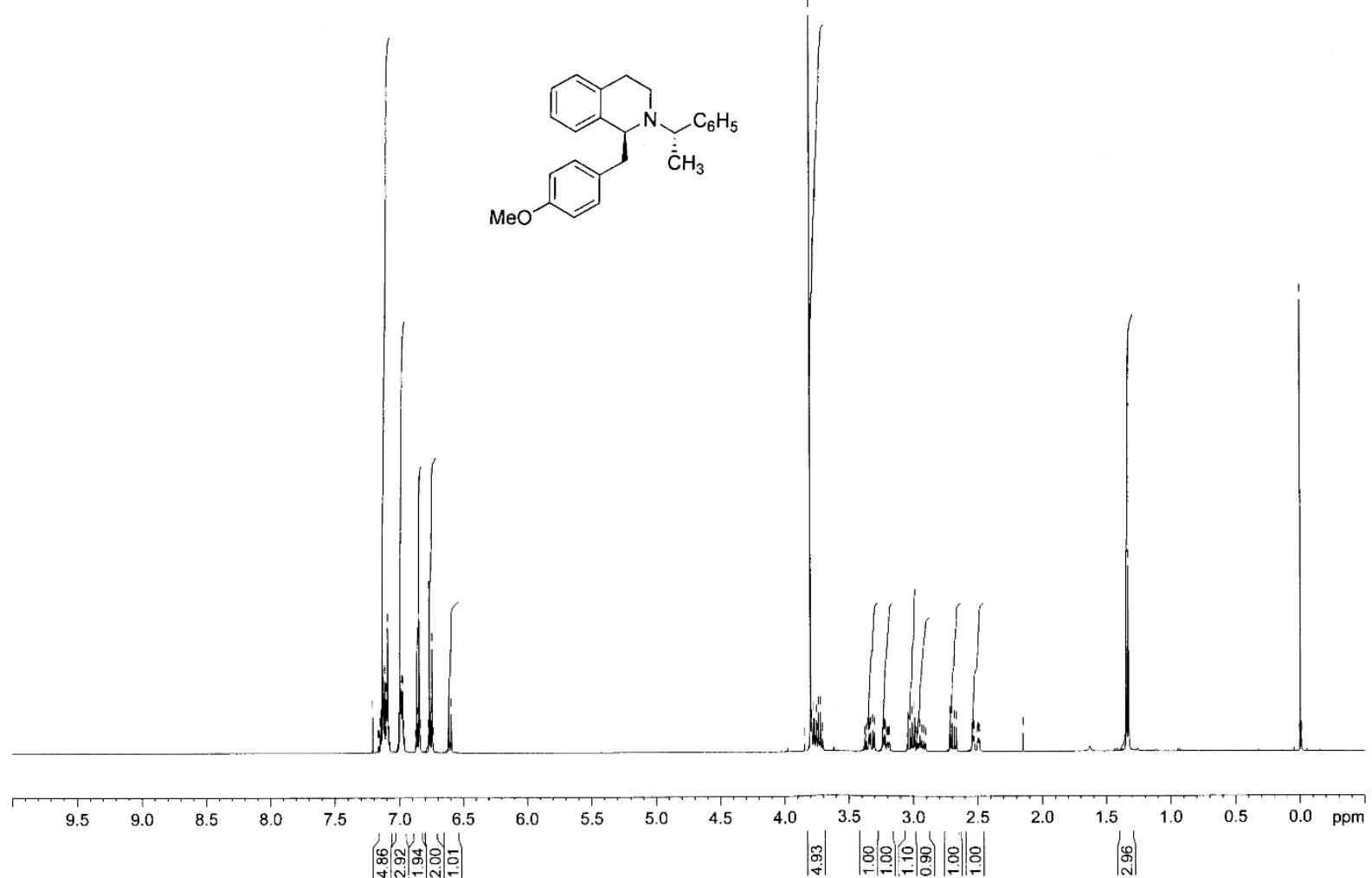

Figure S 44. ${ }^{1} \mathrm{H}$ NMR spectrum $\left(\mathrm{CDCl}_{3}, 400 \mathrm{MHz}\right)$ of derivative (-)-1f. 


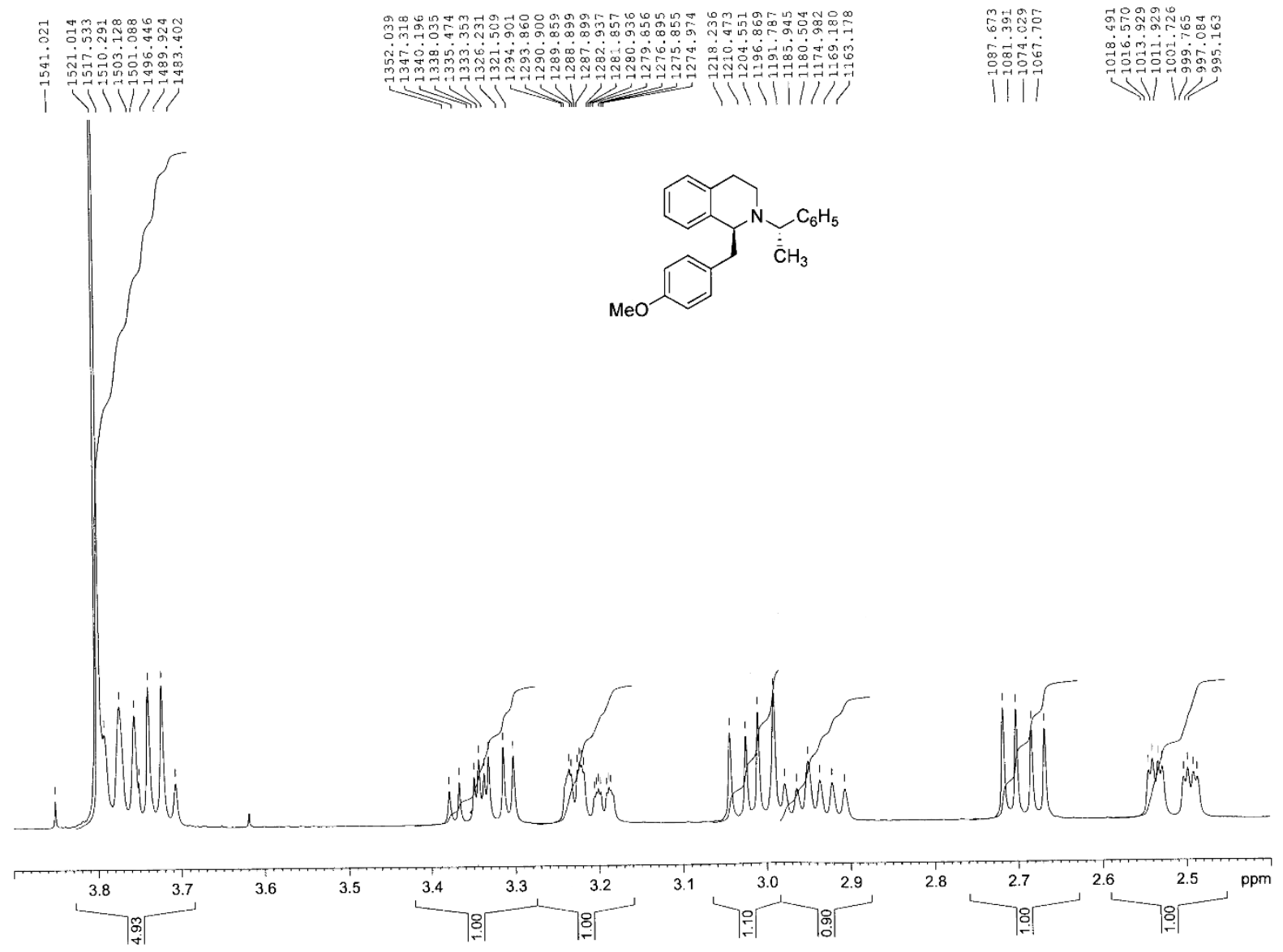

Figure S 45. ${ }^{1} \mathrm{H}$ NMR spectrum $\left(\mathrm{CDCl}_{3}, 400 \mathrm{MHz}\right)$ of derivative (+)-1f. 
<smiles>COc1ccc(CC2c3ccccc3CCN2C(C)c2ccccc2)cc1</smiles>
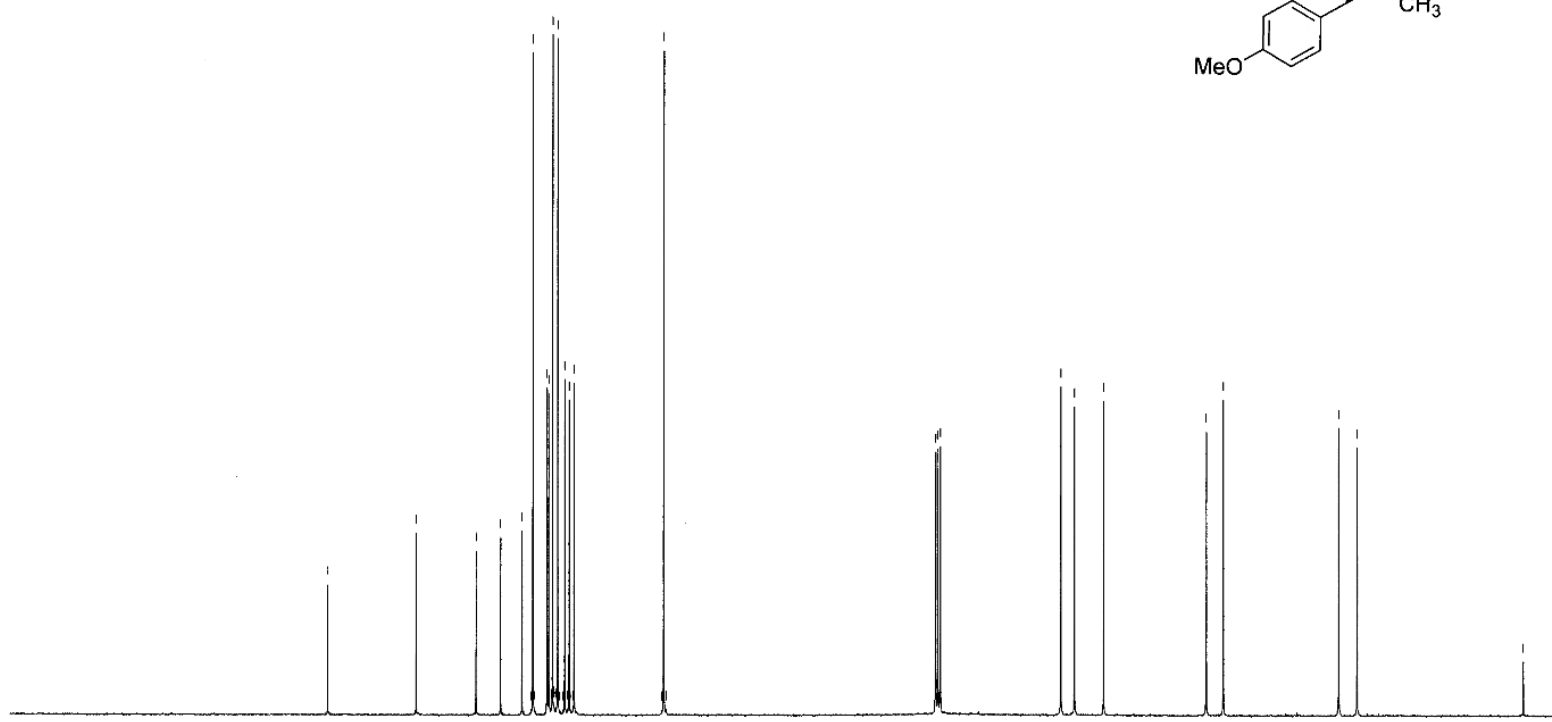

$190 \quad 180 \quad 170$

Figure $\mathbf{S} 46 .{ }^{13} \mathrm{C}$ NMR spectrum $\left(\mathrm{CDCl}_{3}, 100 \mathrm{MHz}\right)$ of derivative (+)-1f. 


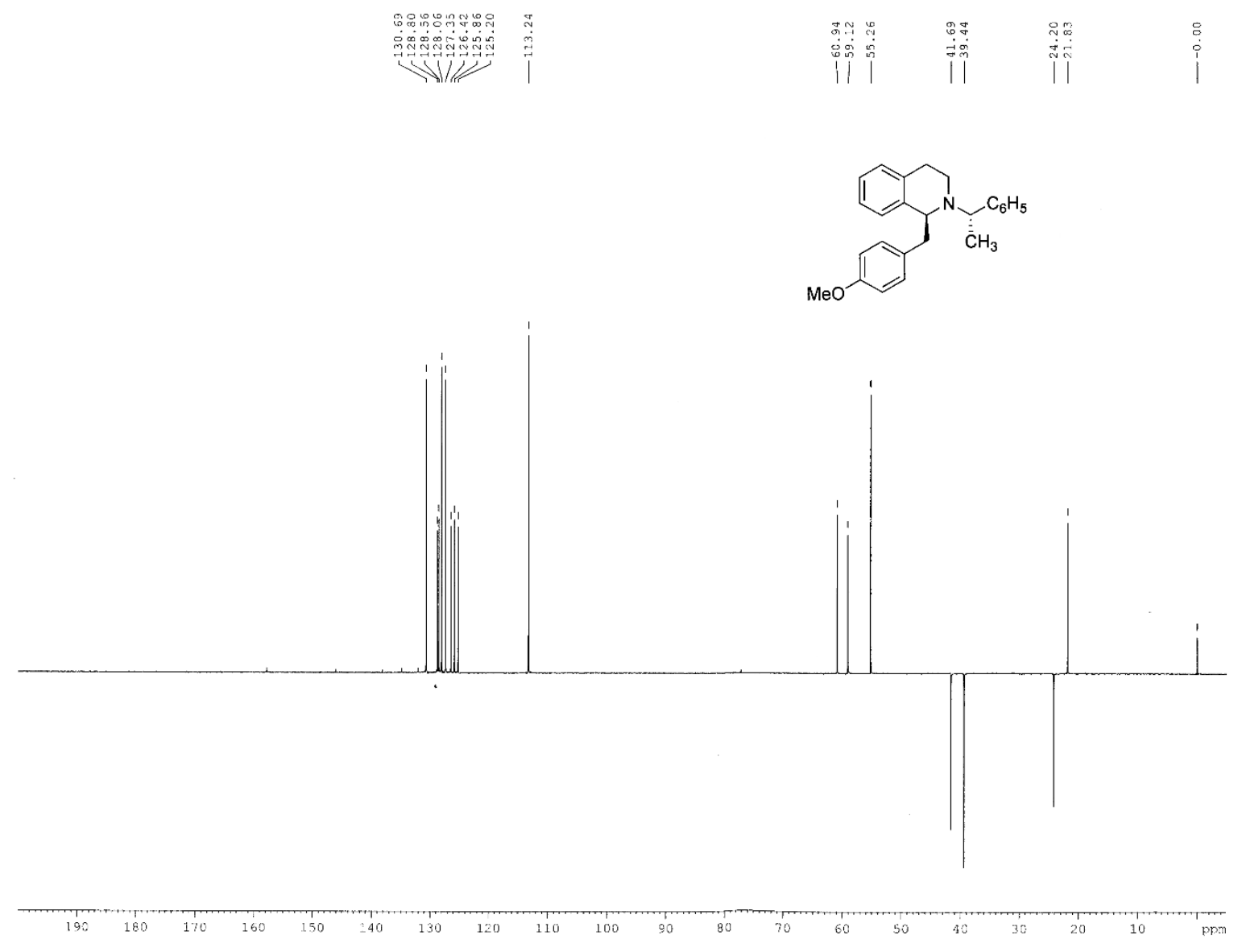

Figure S 47. DEPT C NMR spectrum ( $\left.\mathrm{CDCl}_{3}, 100 \mathrm{MHz}\right)$ of derivative (+)-1f. 


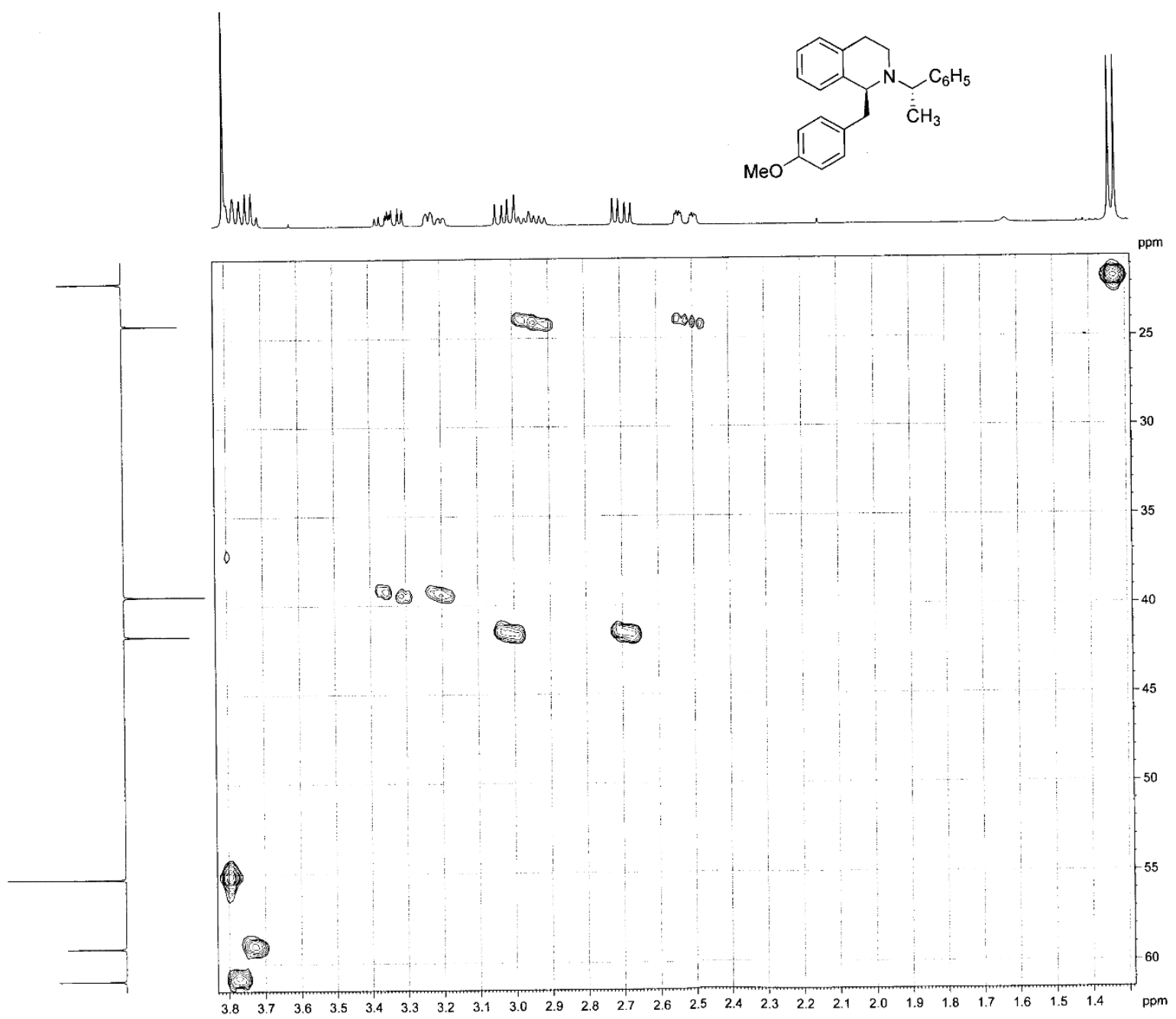

Figure S 48. $\mathrm{HMQC}$ spectrum $\left(\mathrm{CDCl}_{3}\right)$ of derivative $(+)-\mathbf{1 f}$. 


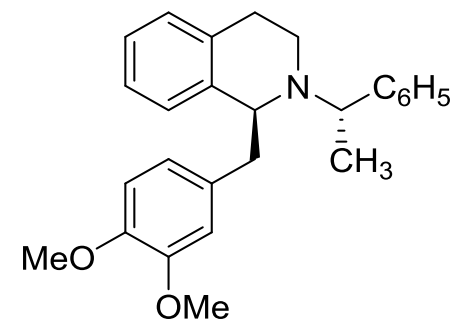

(+)-19

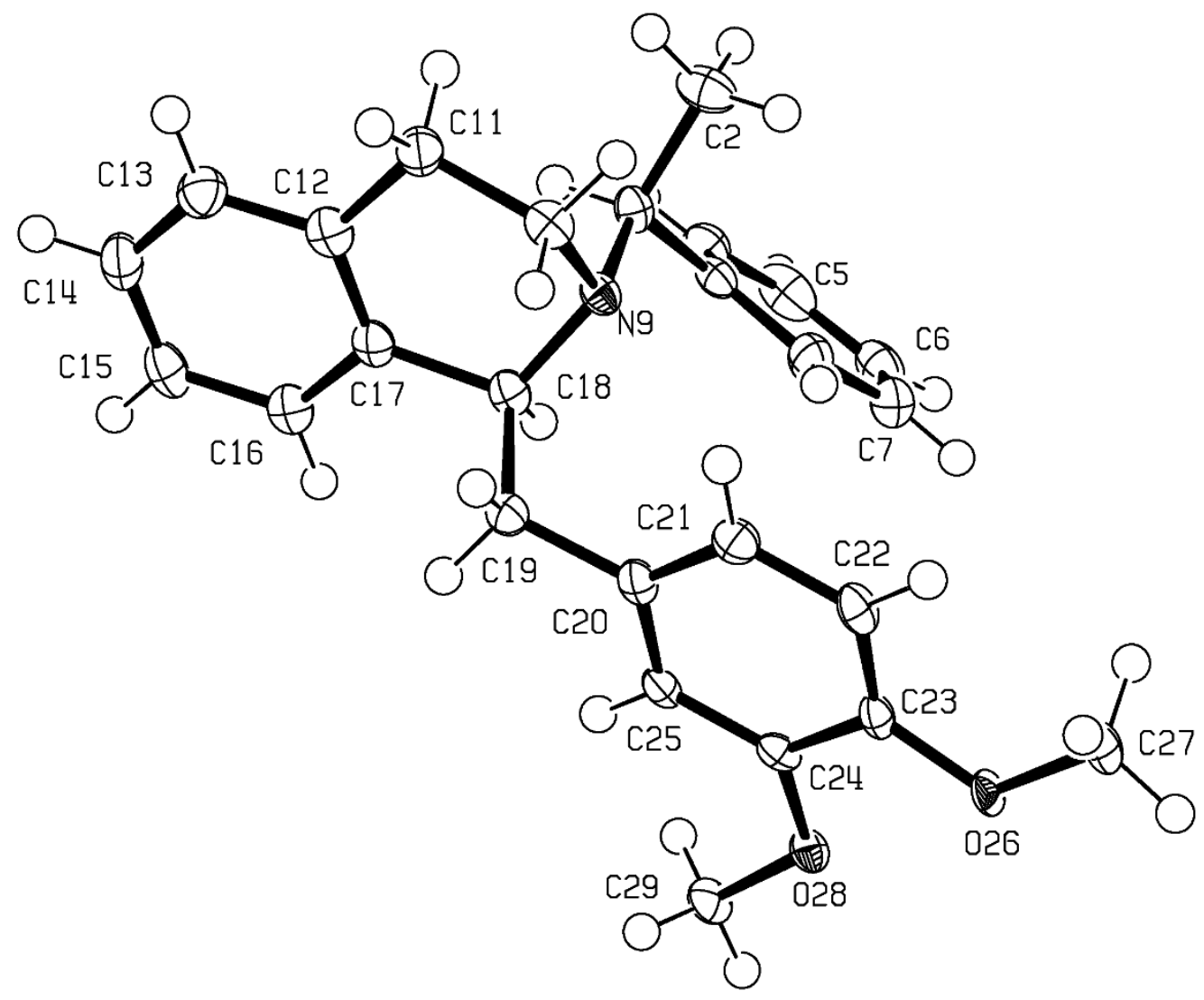

Figure S 49. ORTEP view of derivative (+)-1g. Ellipsoid plots are drawn with $70 \%$ of probability. 


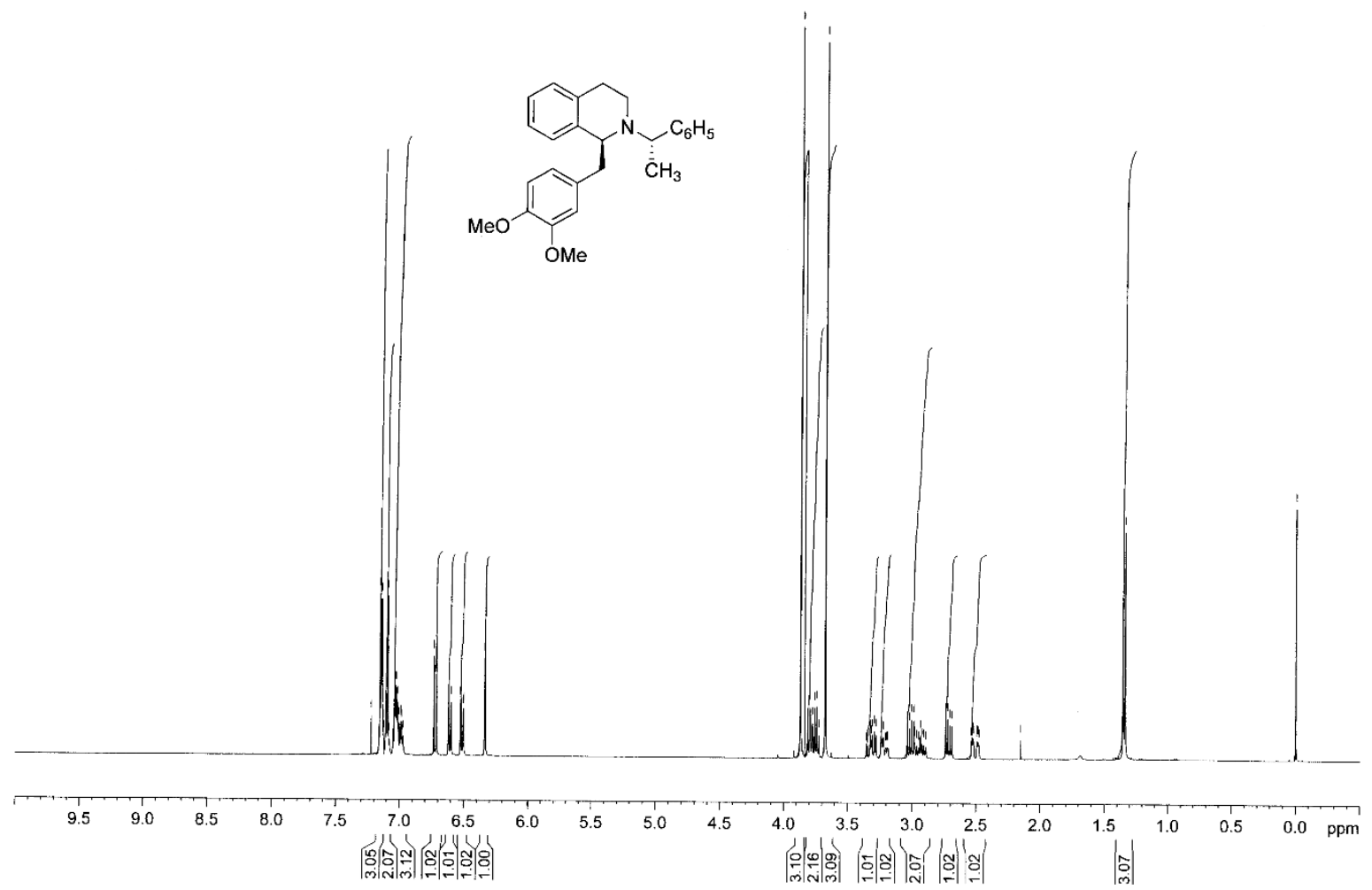

Figure S 50. ${ }^{1} \mathrm{H}$ NMR spectrum $\left(\mathrm{CDCl}_{3}, 400 \mathrm{MHz}\right)$ of derivative (+)-19. 


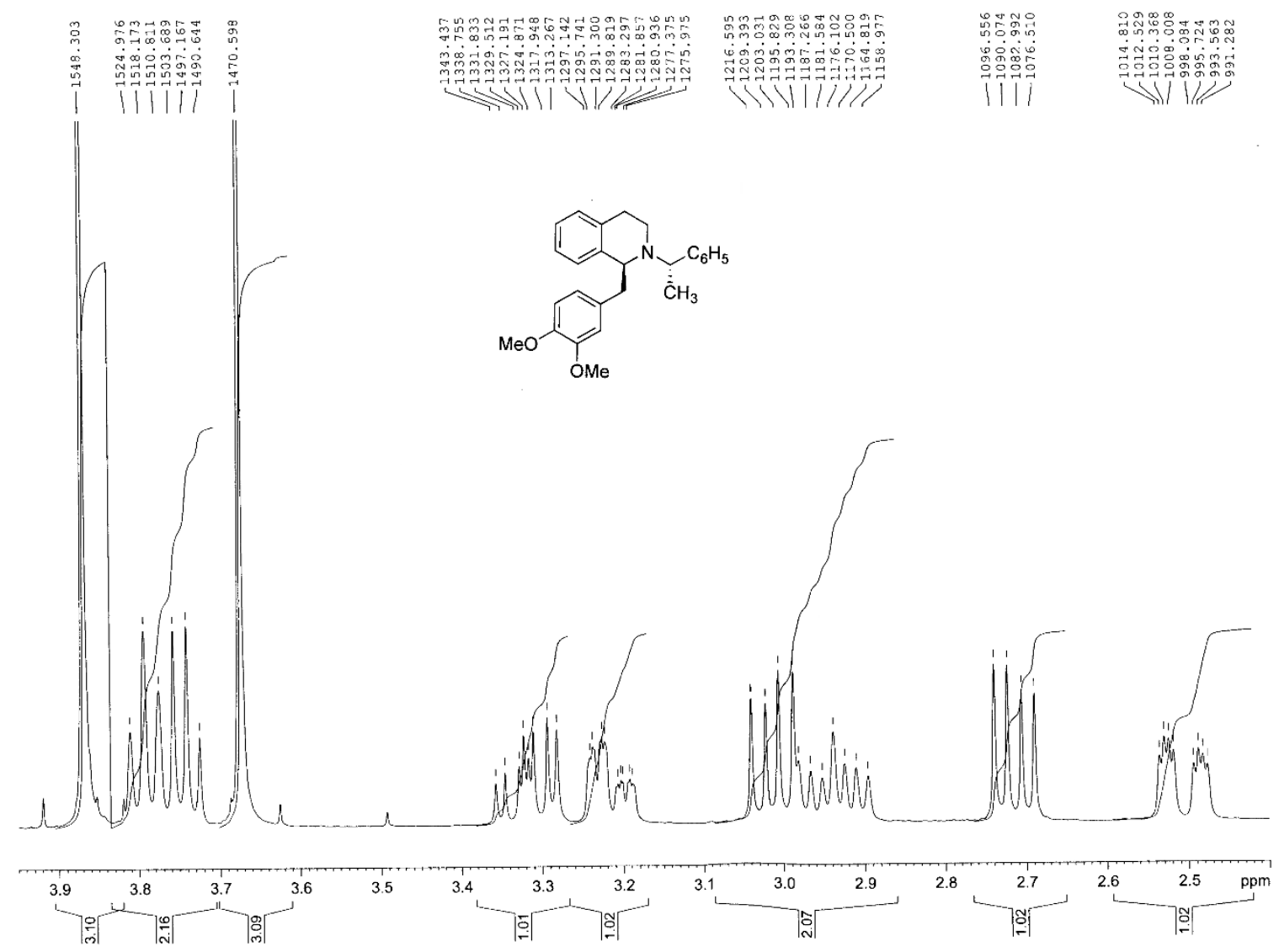

Figure S 51. ${ }^{1} \mathrm{H}$ NMR spectrum $\left(\mathrm{CDCl}_{3}, 400 \mathrm{MHz}\right)$ of derivative (+)-19. 


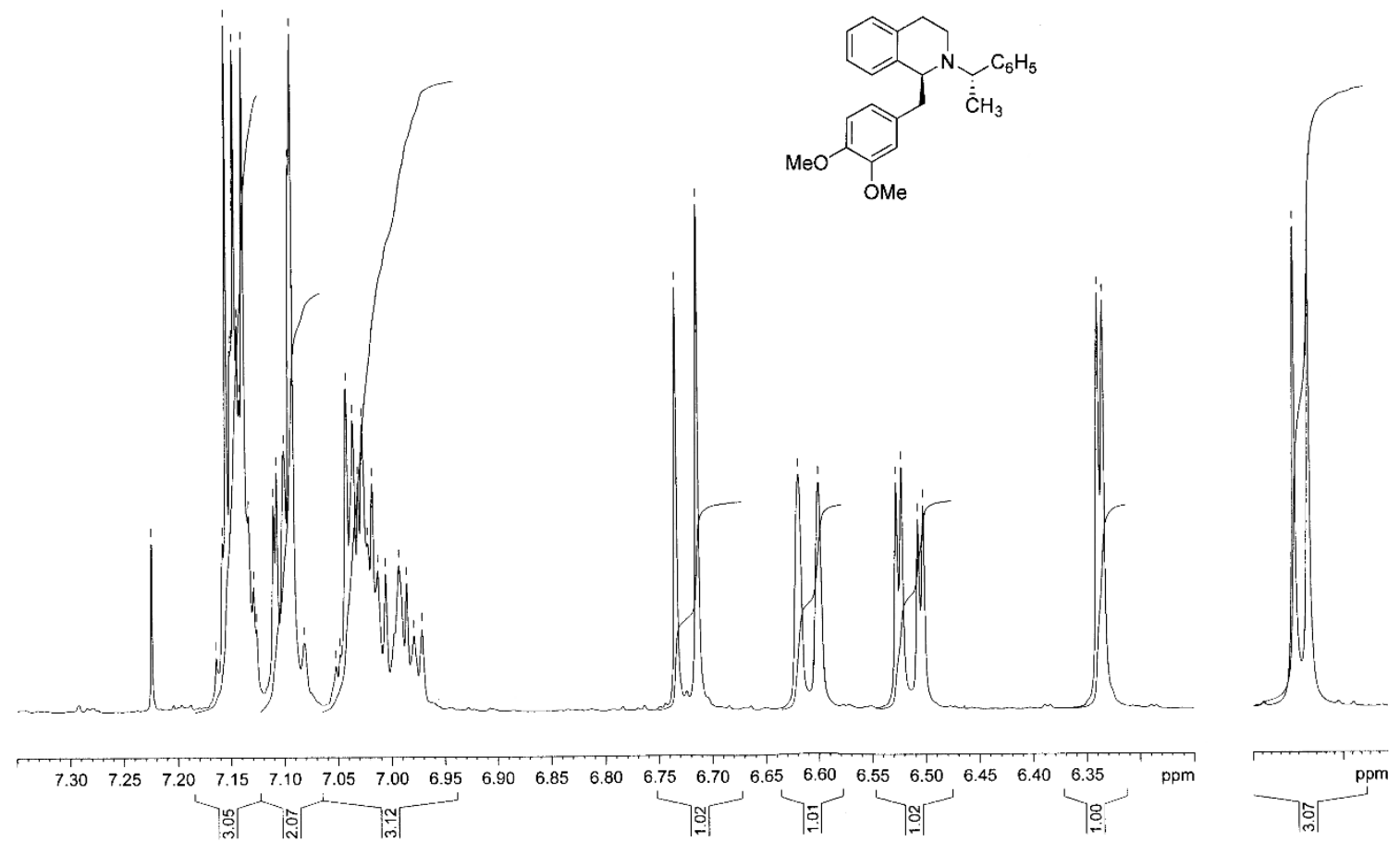

Figure S 52. ${ }^{1} \mathrm{H} \mathrm{NMR}$ spectrum $\left(\mathrm{CDCl}_{3}, 400 \mathrm{MHz}\right)$ of derivative (+)-19. 


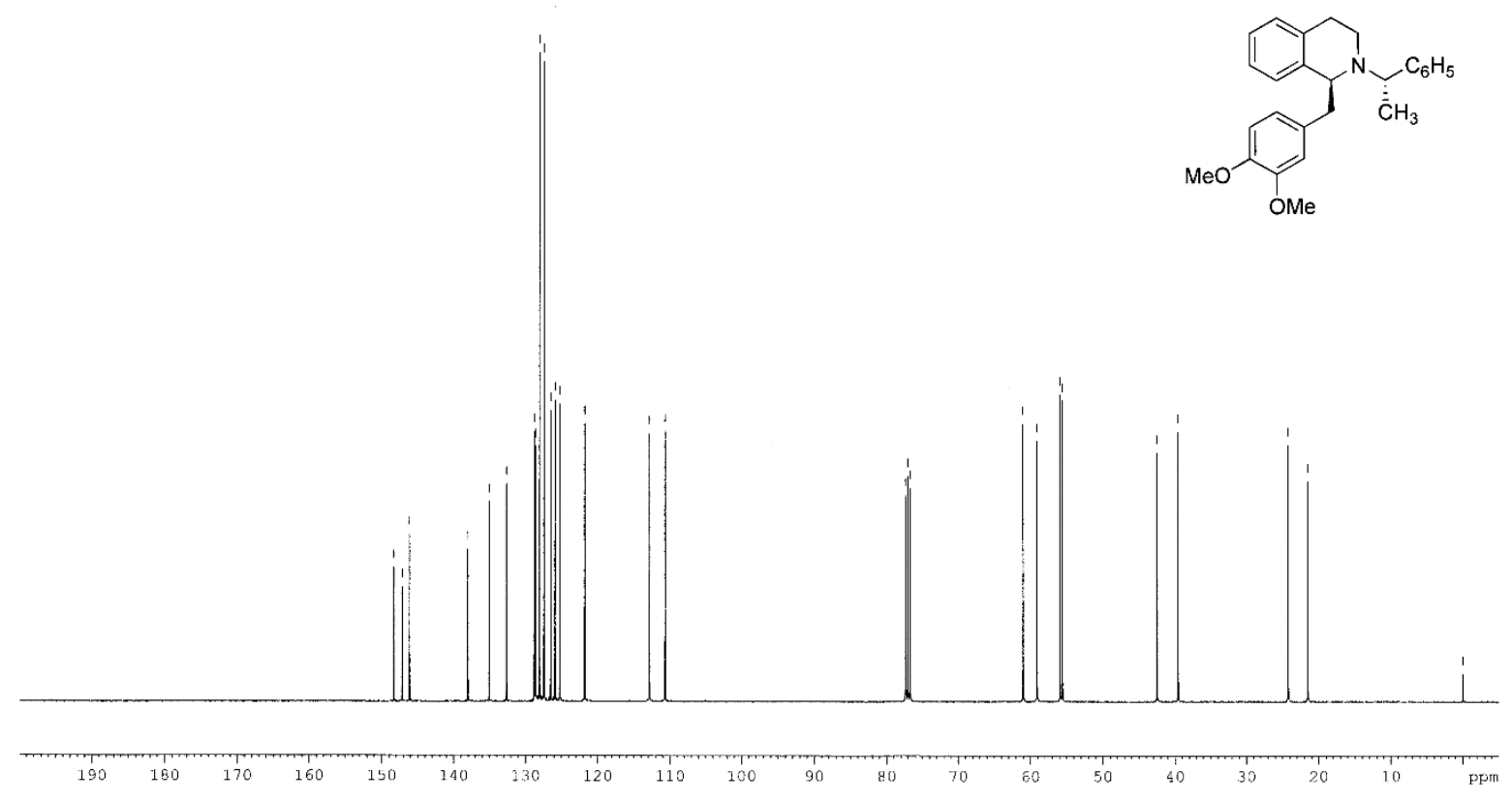

Figure S 53. ${ }^{13} \mathrm{C}$ NMR spectrum $\left(\mathrm{CDCl}_{3}, 100 \mathrm{MHz}\right)$ of derivative $(+)-\mathbf{1 g}$. 


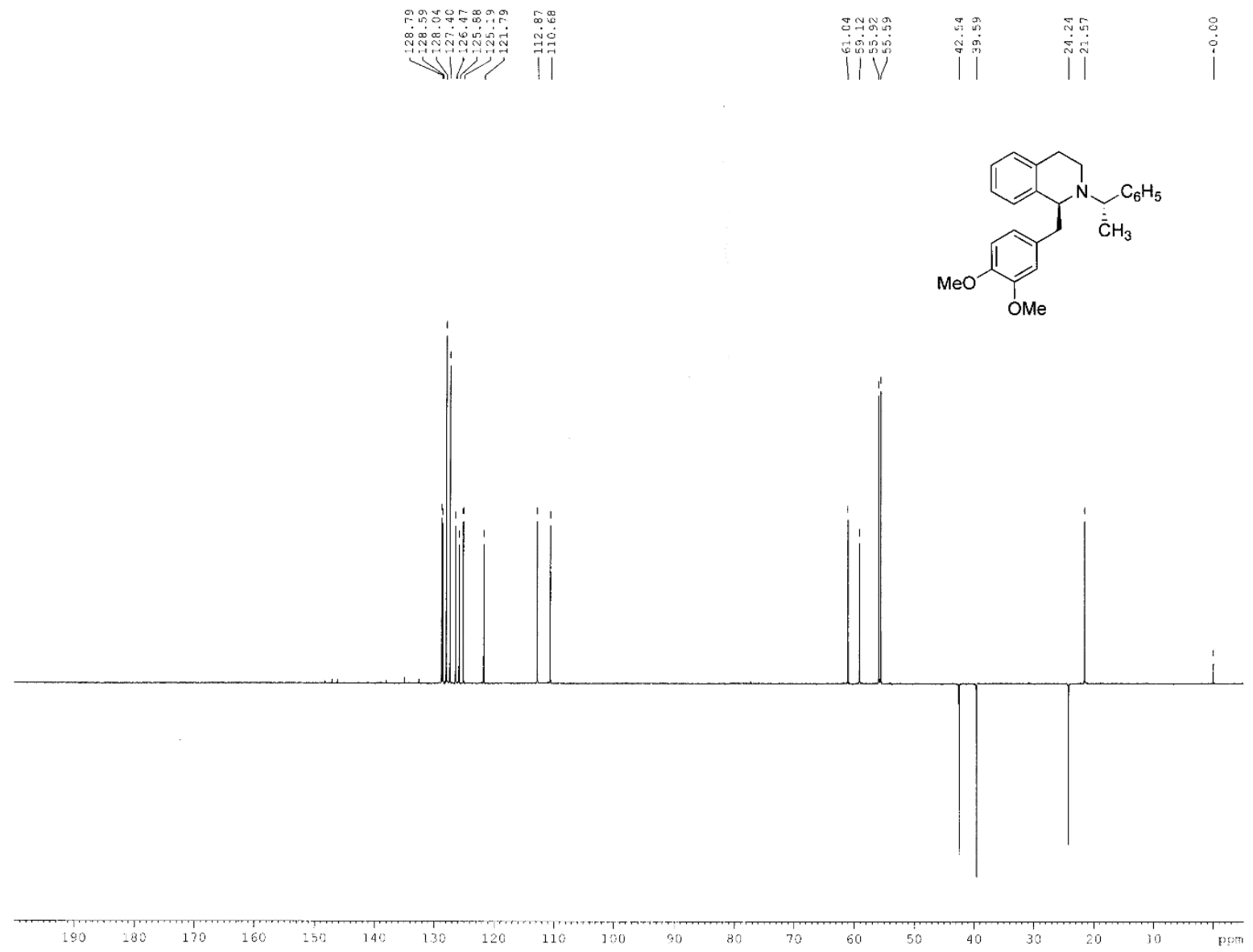

Figure S 54. DEPT C NMR spectrum $\left(\mathrm{CDCl}_{3}, 100 \mathrm{MHz}\right)$ of derivative (+)-1g. 


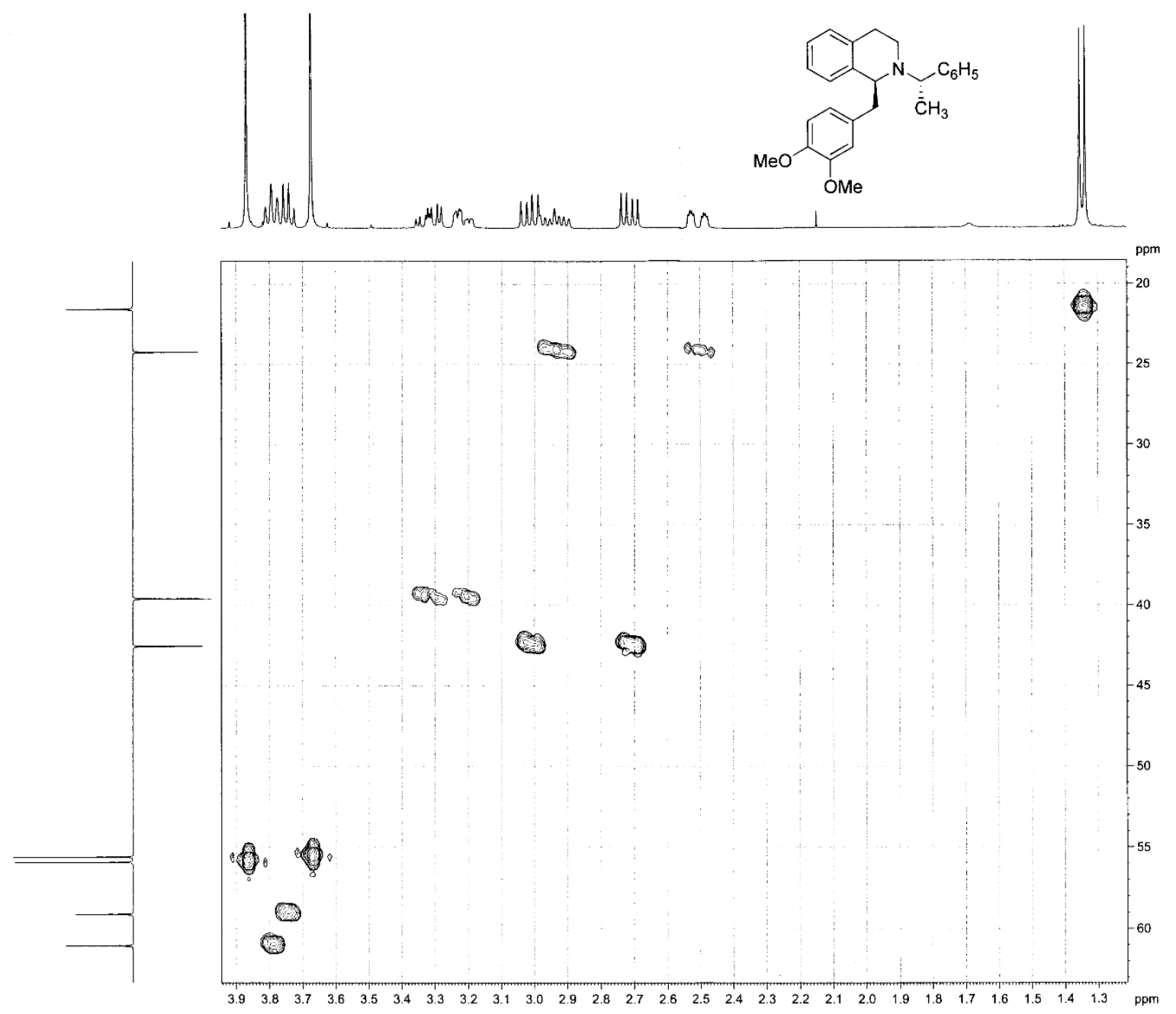

Figure S 55. HMQC spectrum $\left(\mathrm{CDCl}_{3}\right)$ of derivative (+)-1g. 


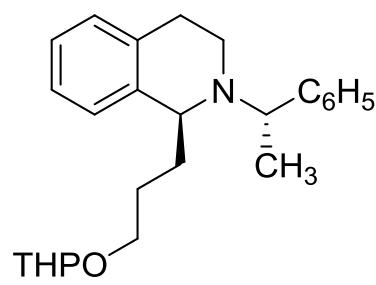

$(-)-1 h$

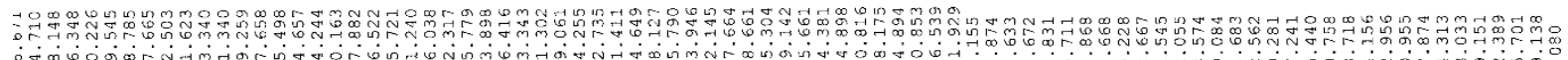
o

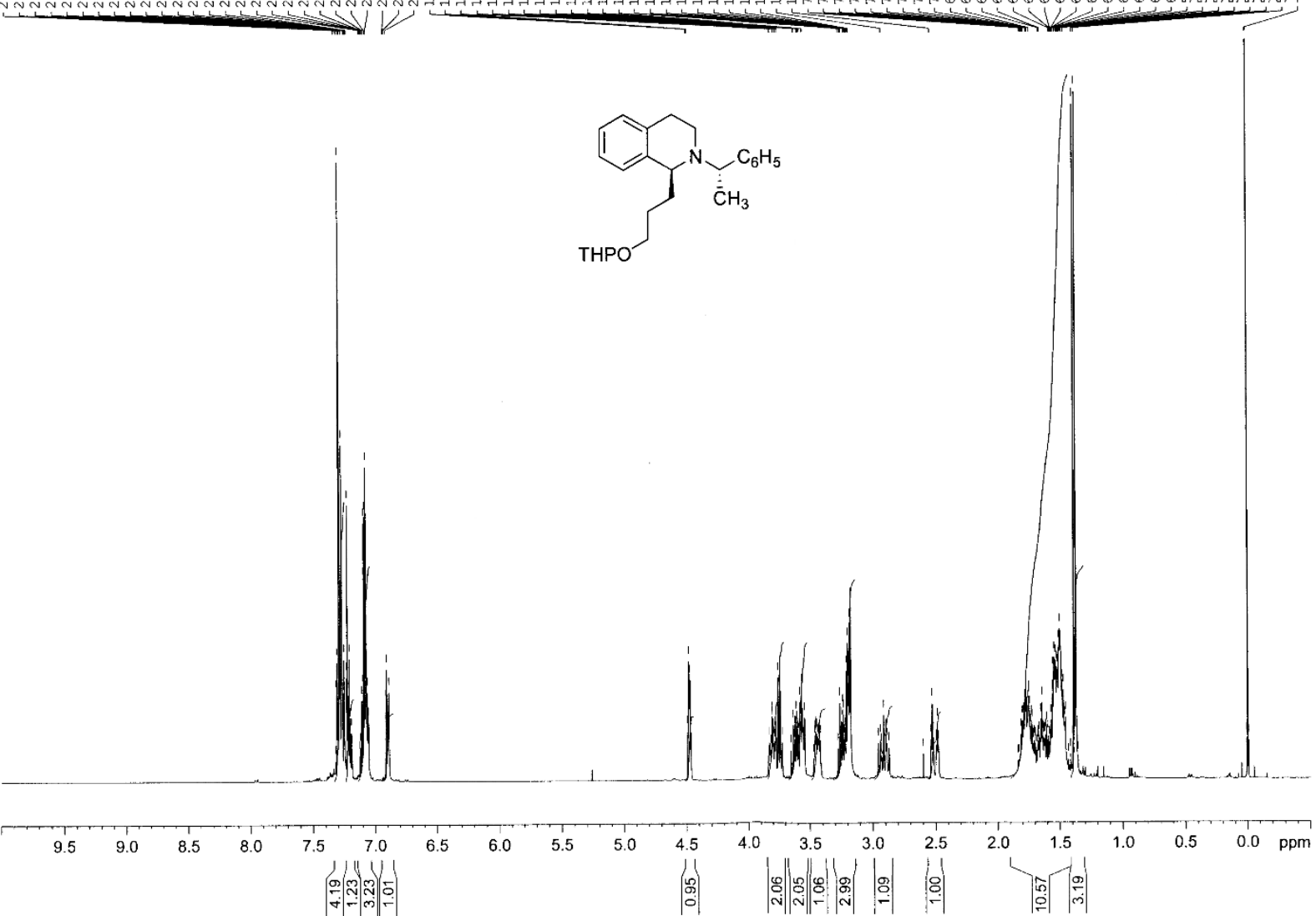

Figure S 56. ${ }^{1} \mathrm{H}$ NMR spectrum $\left(\mathrm{CDCl}_{3}, 400 \mathrm{MHz}\right)$ of derivative (-)-1 h. 
S59
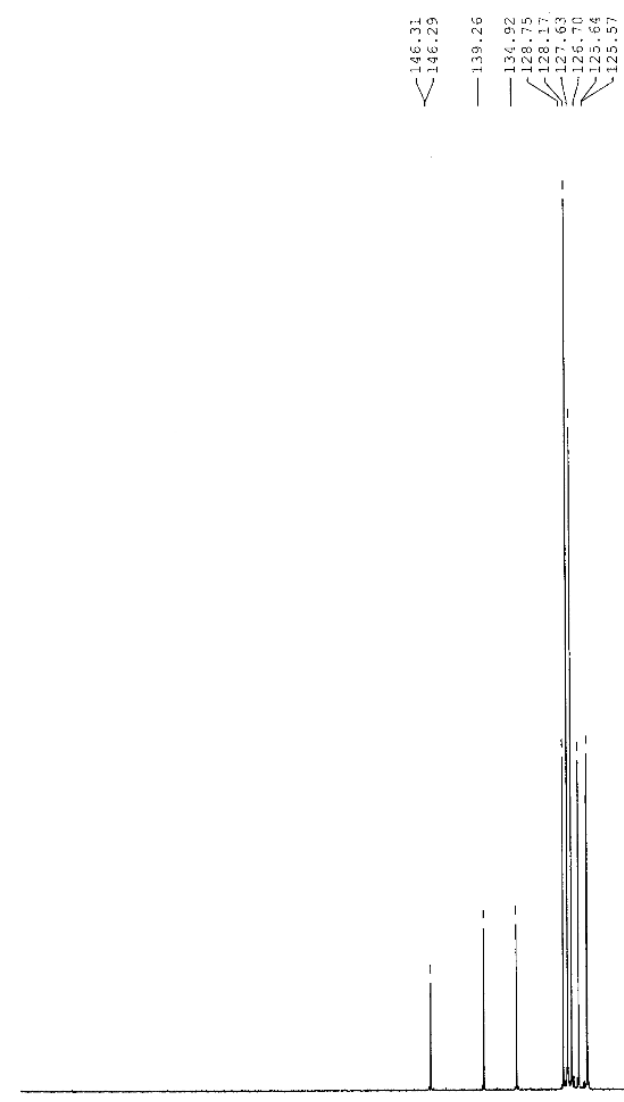
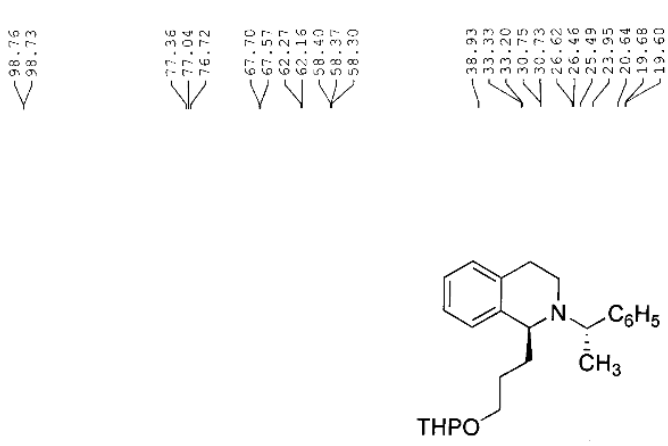

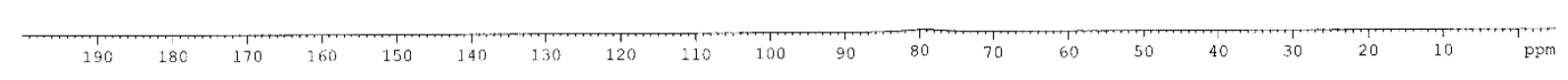

Figure S 57. ${ }^{13} \mathrm{C}$ NMR spectrum $\left(\mathrm{CDCl}_{3}, 100 \mathrm{MHz}\right)$ of derivative (-)-rh. 
<smiles>CC(c1ccccc1)N1CCc2ccccc2C1CCCO[InH]</smiles>

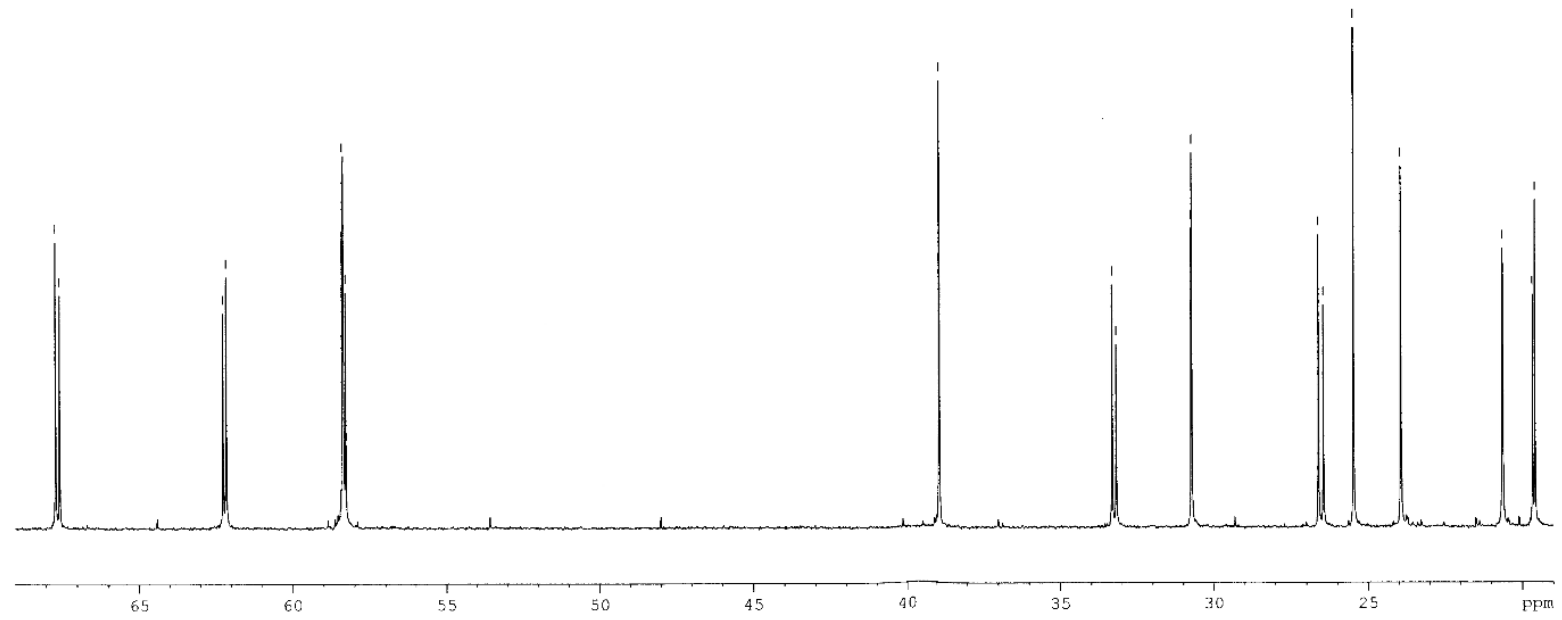

Figure S 58. ${ }^{13} \mathrm{C}$ NMR spectrum $\left(\mathrm{CDCl}_{3}, 100 \mathrm{MHz}\right)$ of derivative $(-)-\mathbf{1} \mathbf{h}$. 
V\}

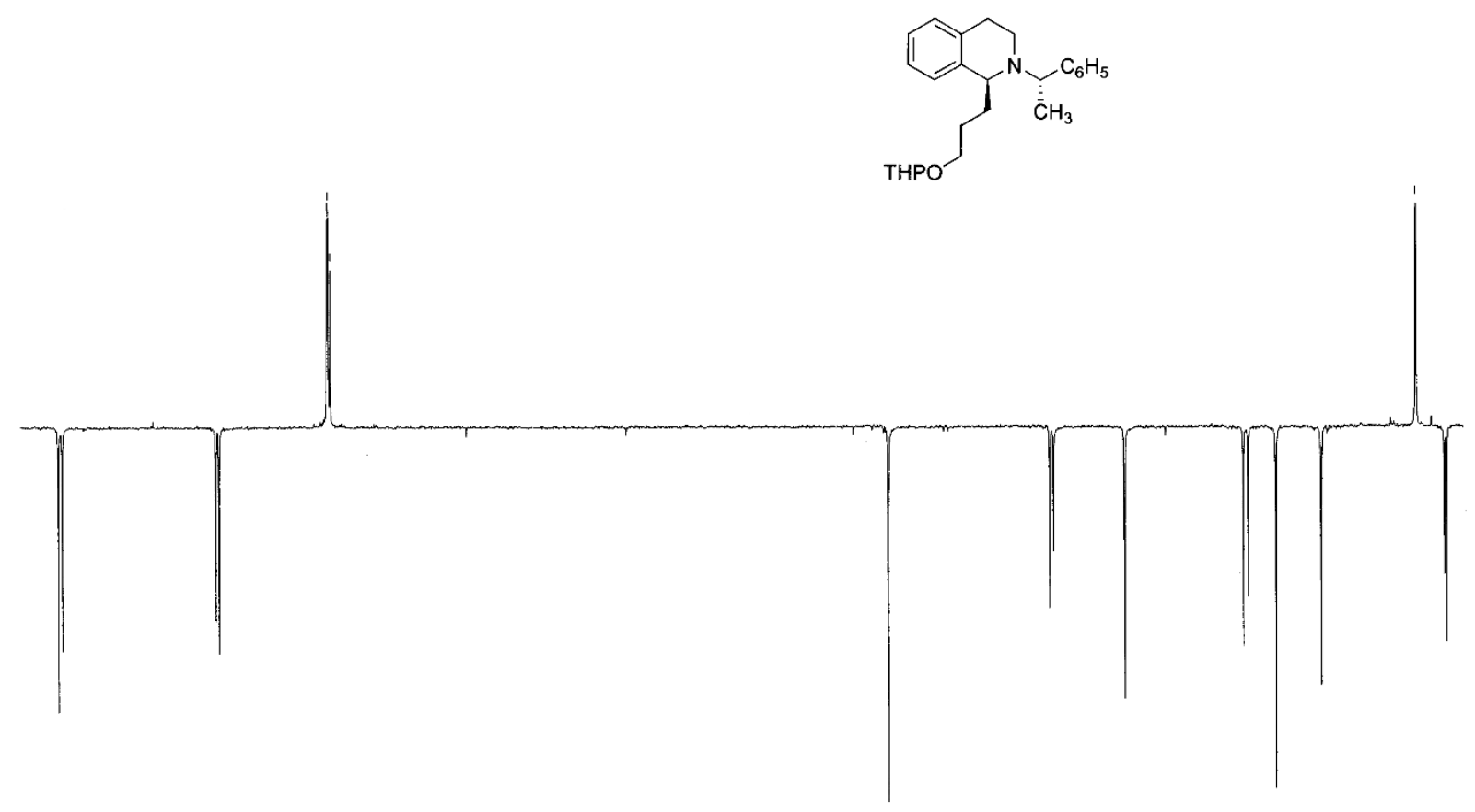

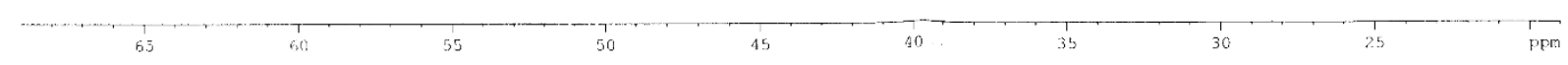

Figure S 59. DEPT C-NMR spectrum $\left(\mathrm{CDCl}_{3}, 100 \mathrm{MHz}\right)$ of derivative (-)-1h. 


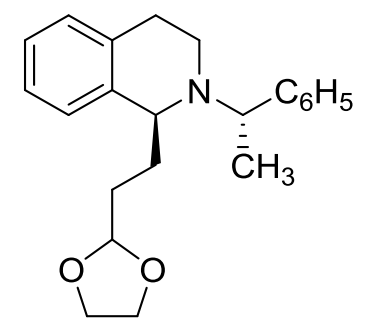

\section{$(-)-\mathbf{1 i}$}

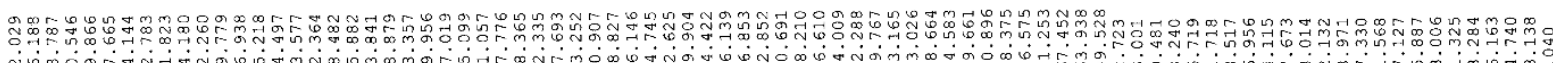

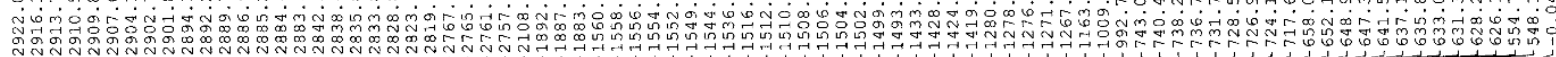

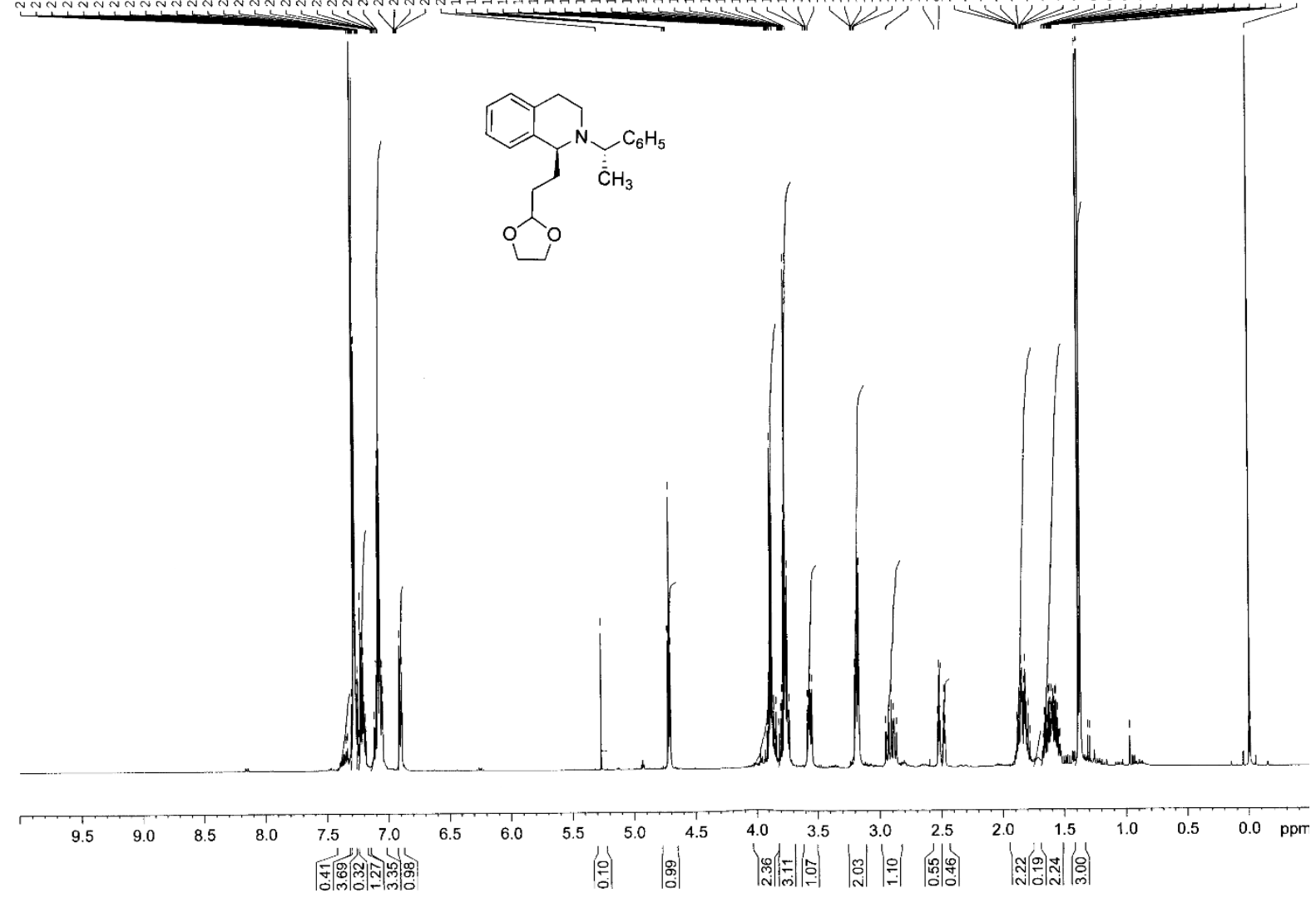

Figure S 60. ${ }^{1} \mathrm{H}$ NMR spectrum $\left(\mathrm{CDCl}_{3}, 400 \mathrm{MHz}\right)$ of derivative (-)-1i. 


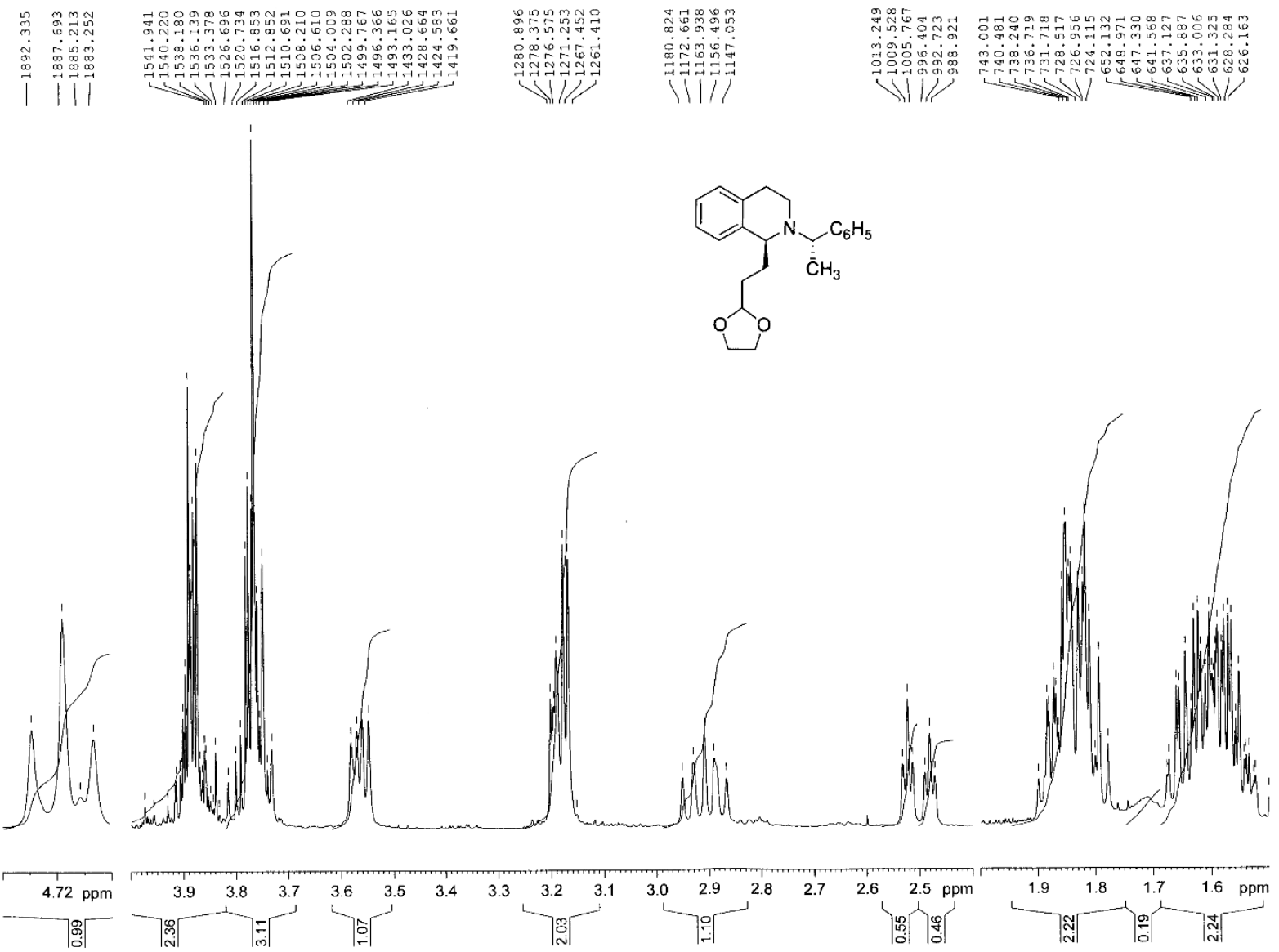

Figure S 61. ${ }^{1} \mathrm{H}$ NMR spectrum $\left(\mathrm{CDCl}_{3}, 400 \mathrm{MHz}\right)$ of derivative (-)-1i. 


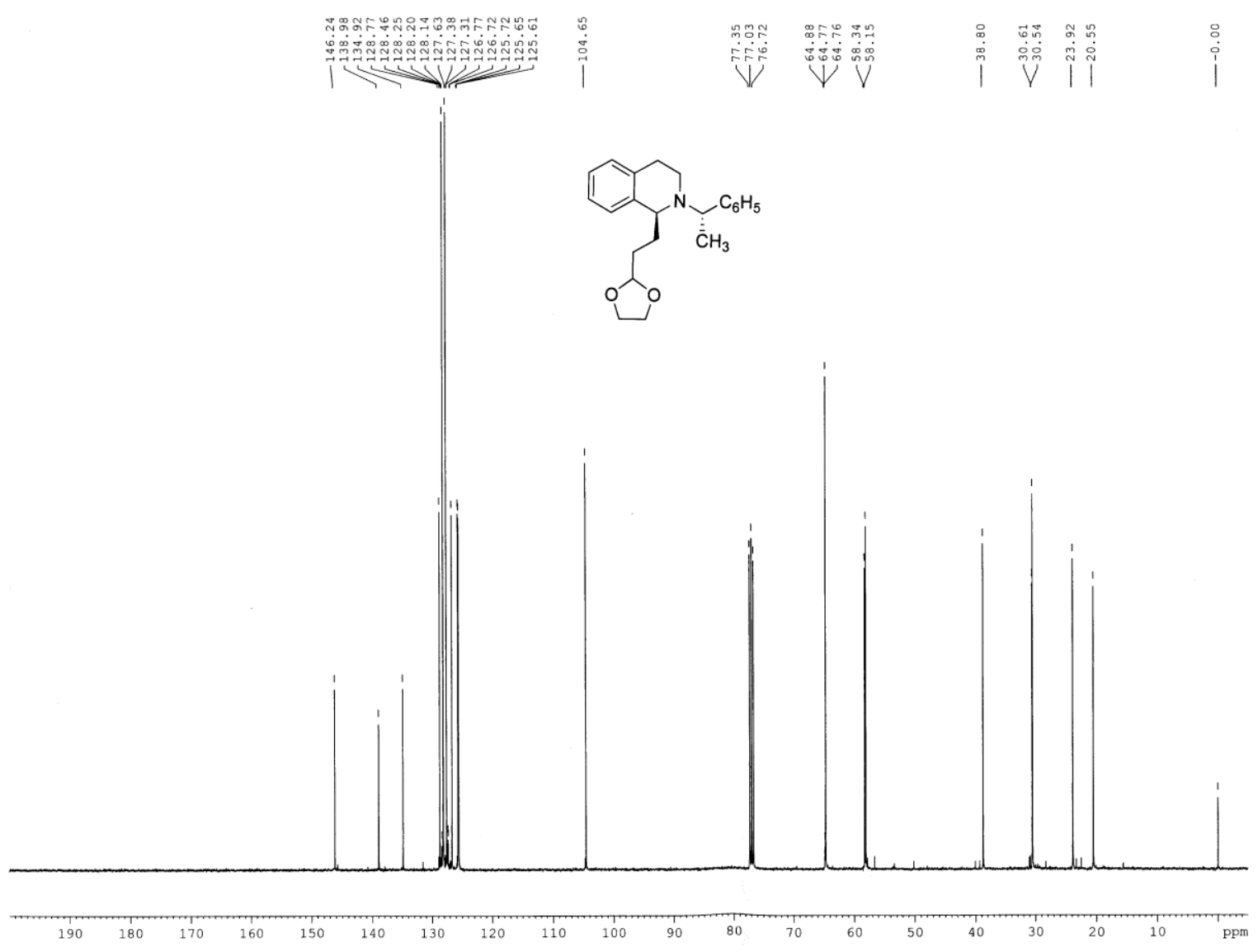

Figure S 62. ${ }^{13} \mathrm{C}$ NMR spectrum $\left(\mathrm{CDCl}_{3}, 100 \mathrm{MHz}\right)$ of derivative (-)-1i. 


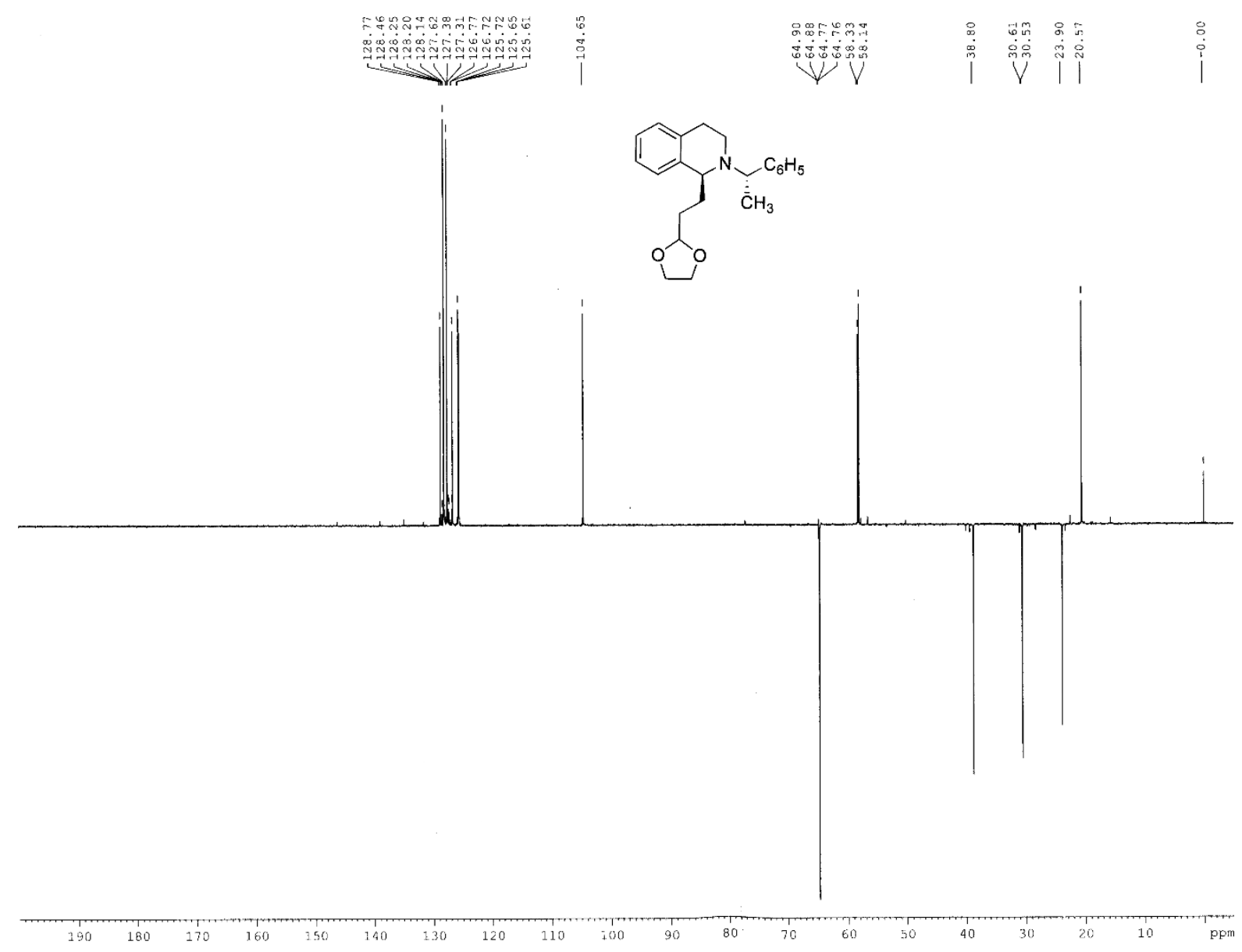

Figure S 63. DEPT C-NMR spectrum $\left(\mathrm{CDCl}_{3}, 100 \mathrm{MHz}\right)$ of derivative (-)-1i. 


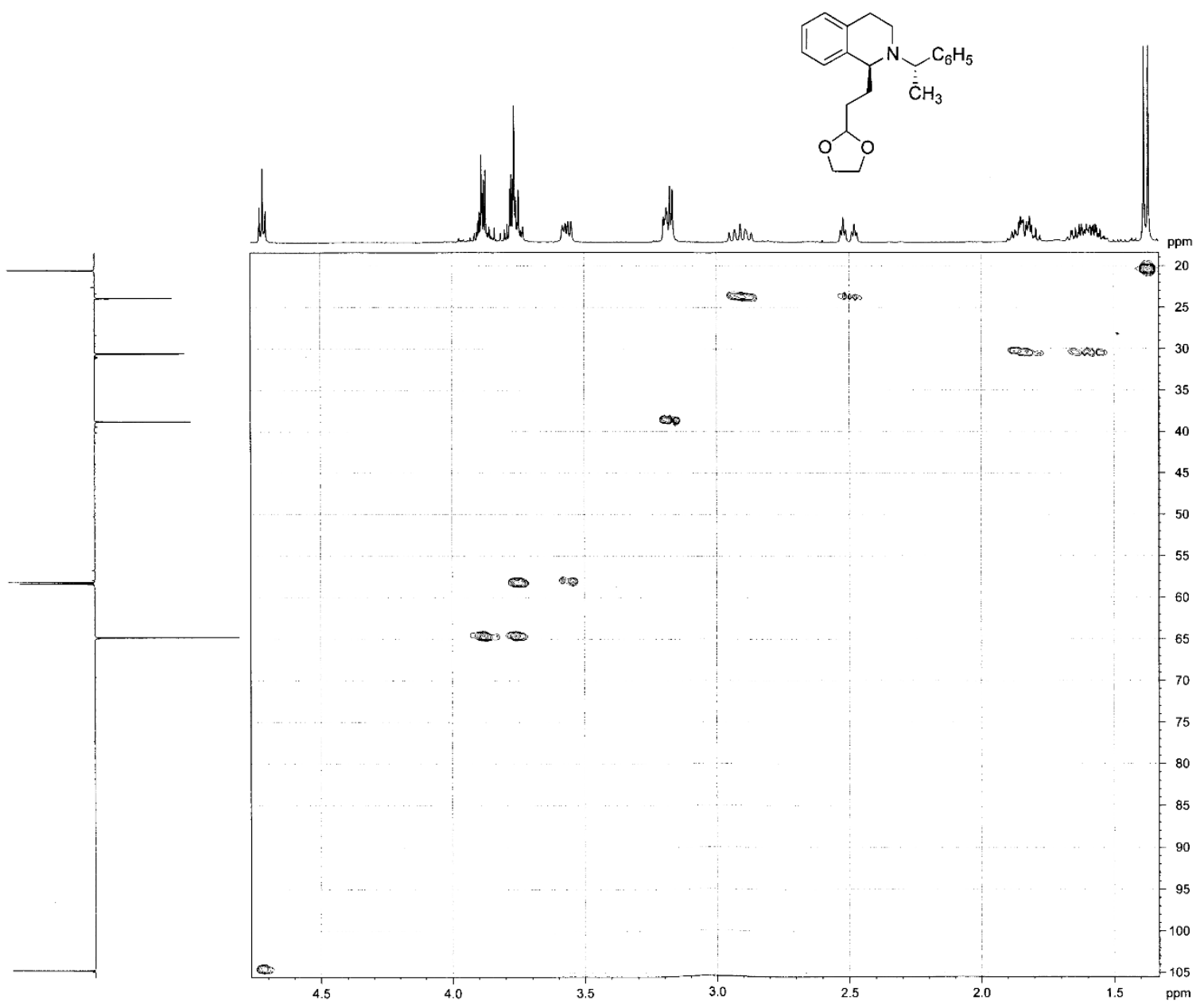

Figure S 64. $\mathrm{HMQC}$ spectrum $\left(\mathrm{CDCl}_{3}\right)$ of derivative (-)-1i. 


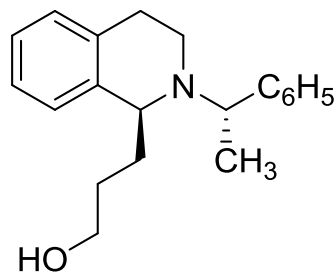

$(-)-\mathbf{j}$

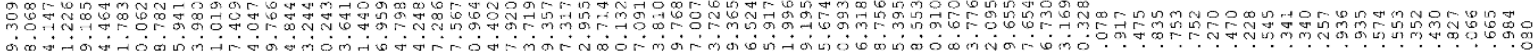

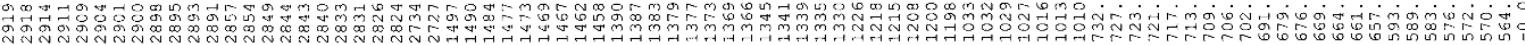

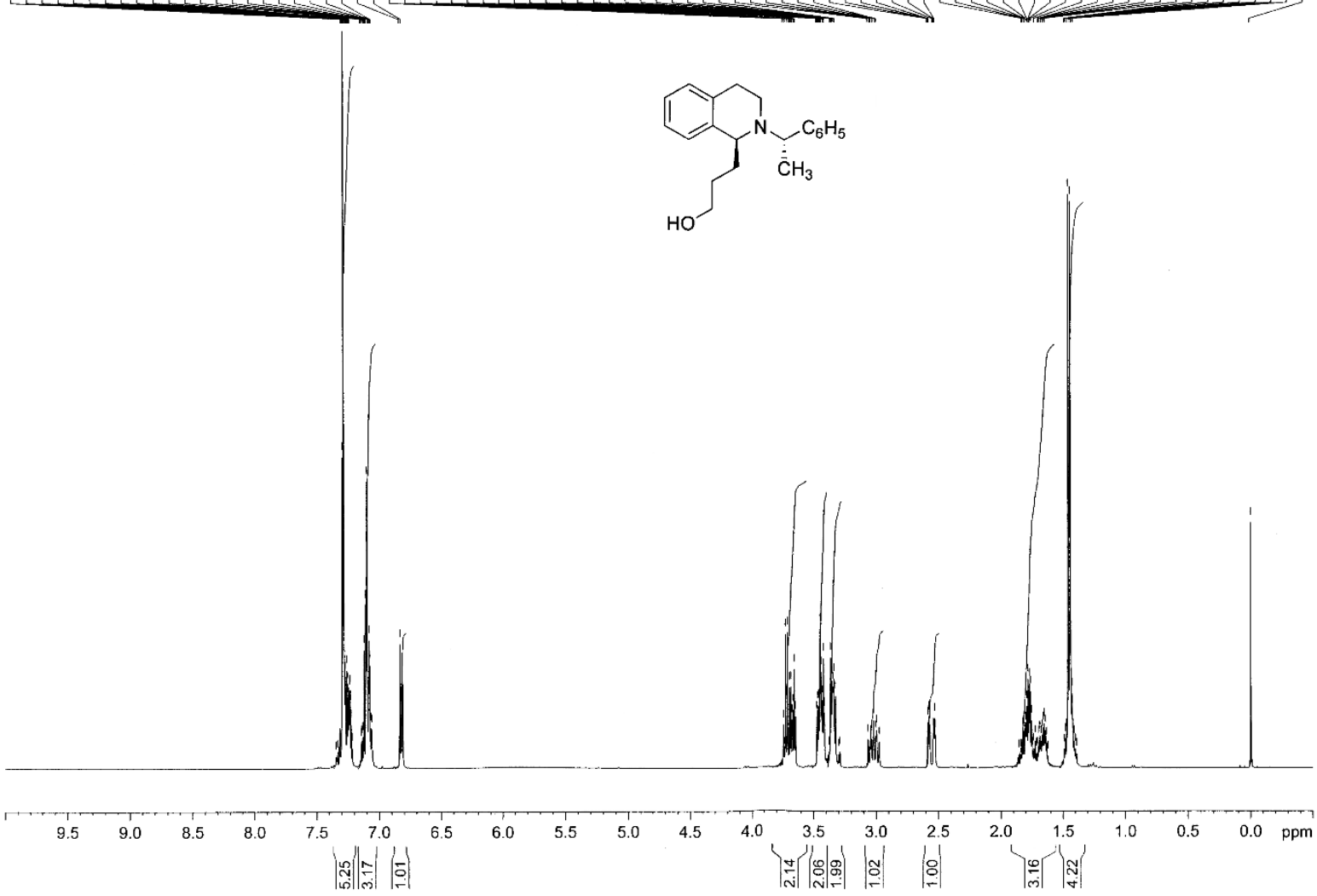

Figure S 65. ${ }^{1} \mathrm{H}$ NMR spectrum $\left(\mathrm{CDCl}_{3}, 400 \mathrm{MHz}\right)$ of derivative (-)-1j. 

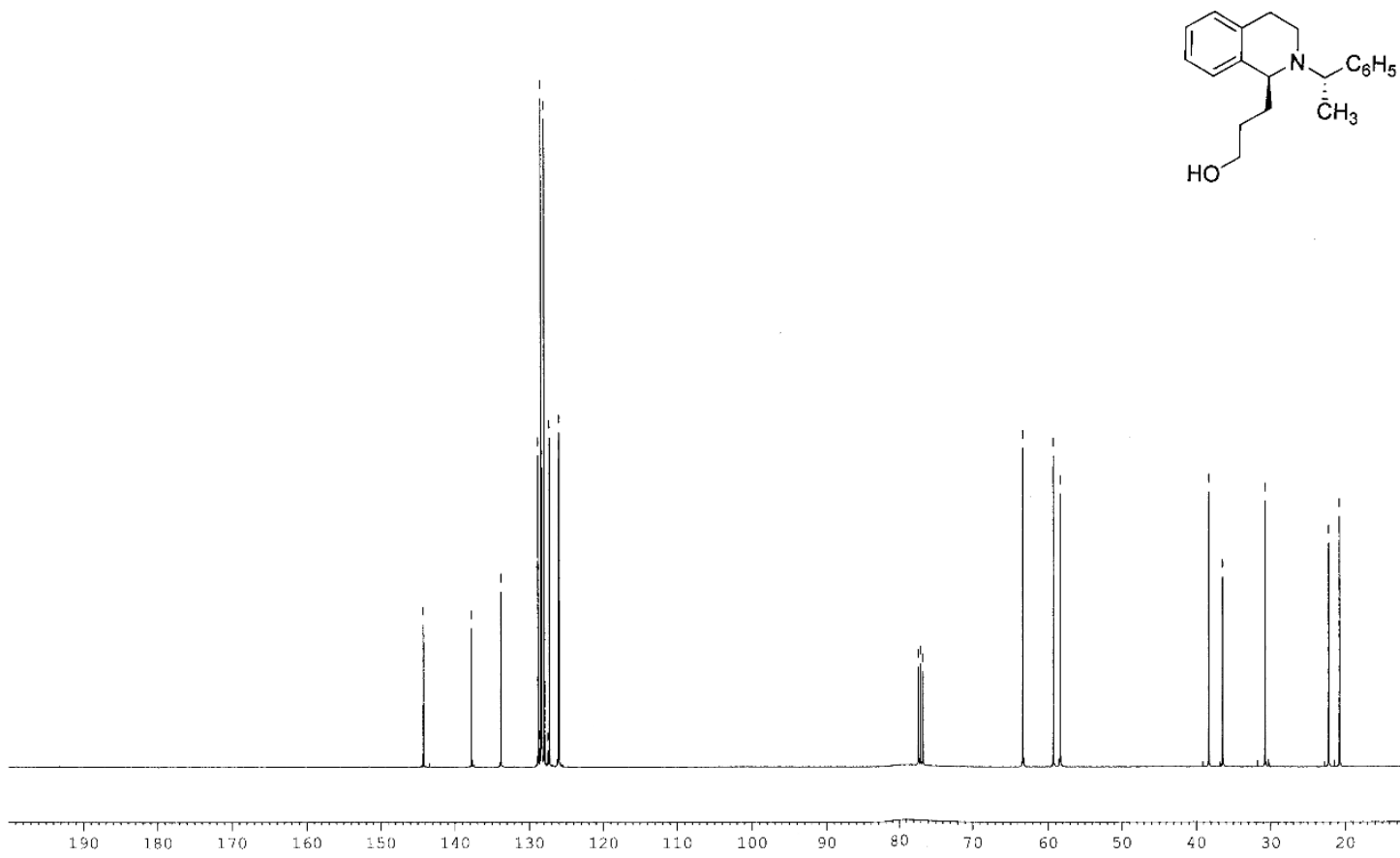

Figure S 66. ${ }^{13} \mathrm{C}$ NMR spectrum $\left(\mathrm{CDCl}_{3}, 100 \mathrm{MHz}\right)$ of derivative $(-)-\mathbf{1} \mathbf{j}$. 


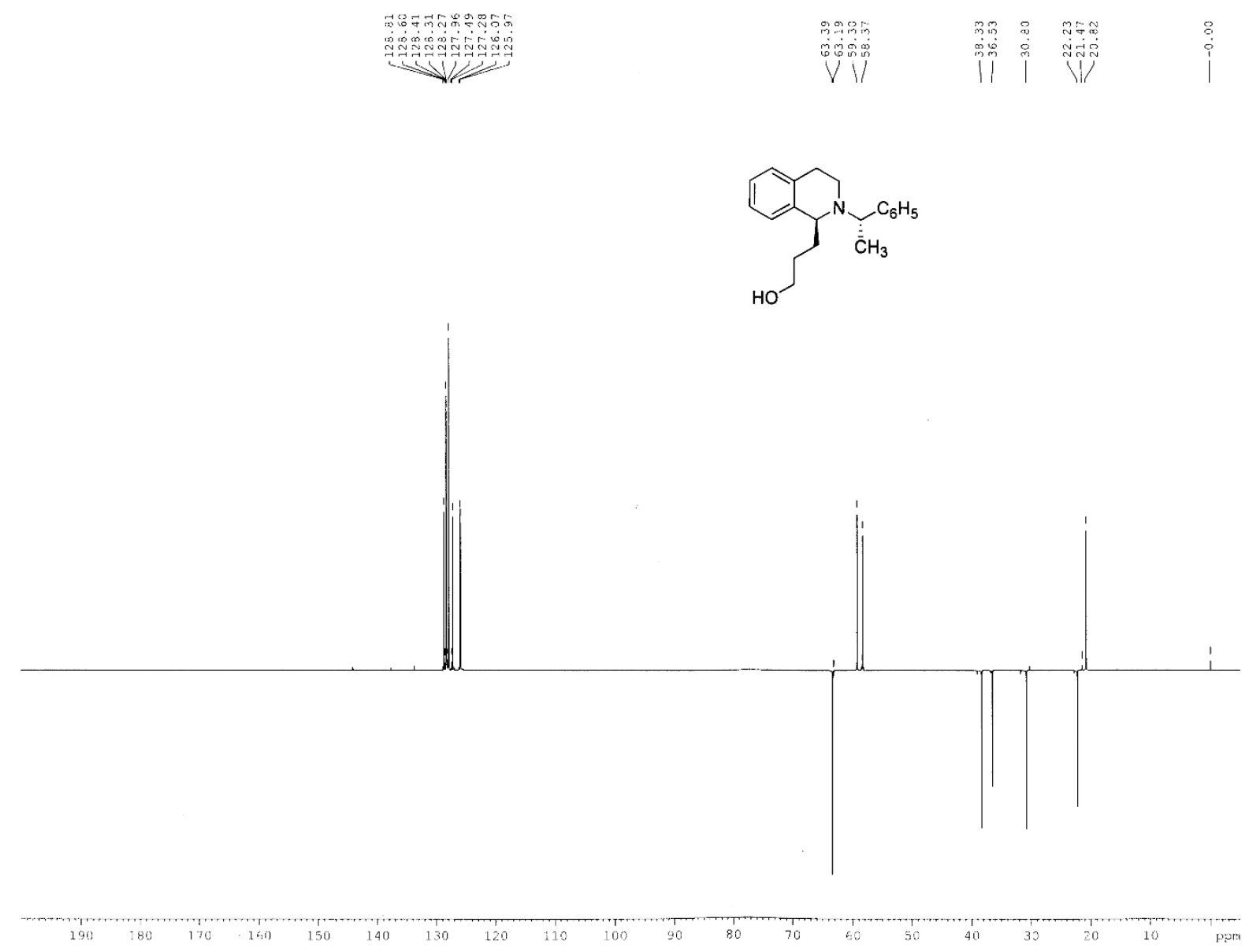

Figure $\mathbf{S}$ 67. DEPT C NMR spectrum $\left(\mathrm{CDCl}_{3}, 100 \mathrm{MHz}\right)$ of derivative (-)-1j. 
S70

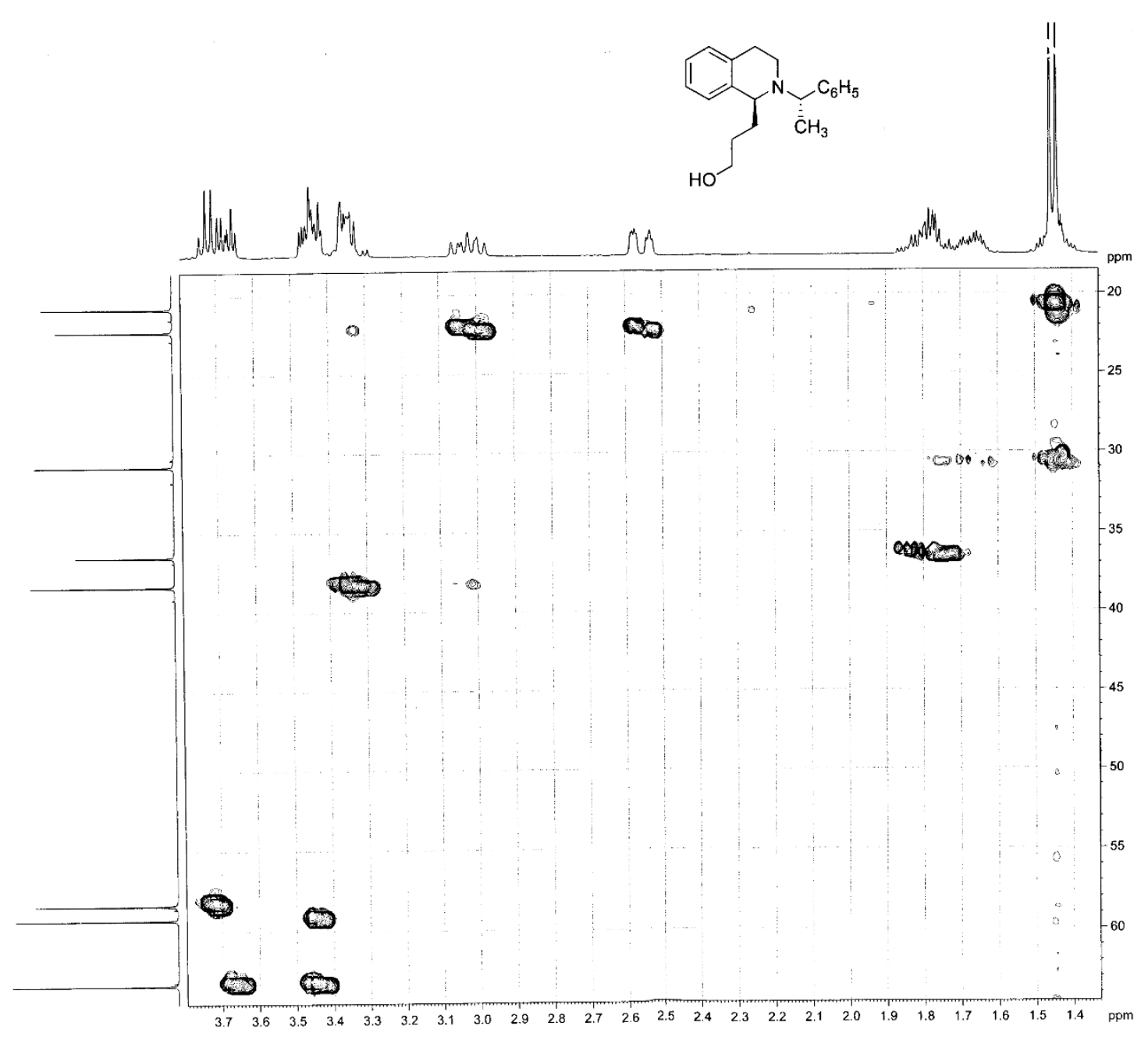

Figure S 68. $\mathrm{HMQC}$ spectrum $\left(\mathrm{CDCl}_{3}\right)$ of derivative (-)-1j. 


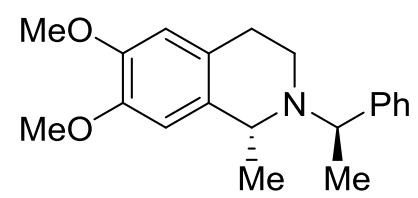

$(+)-1 \mathbf{k}$

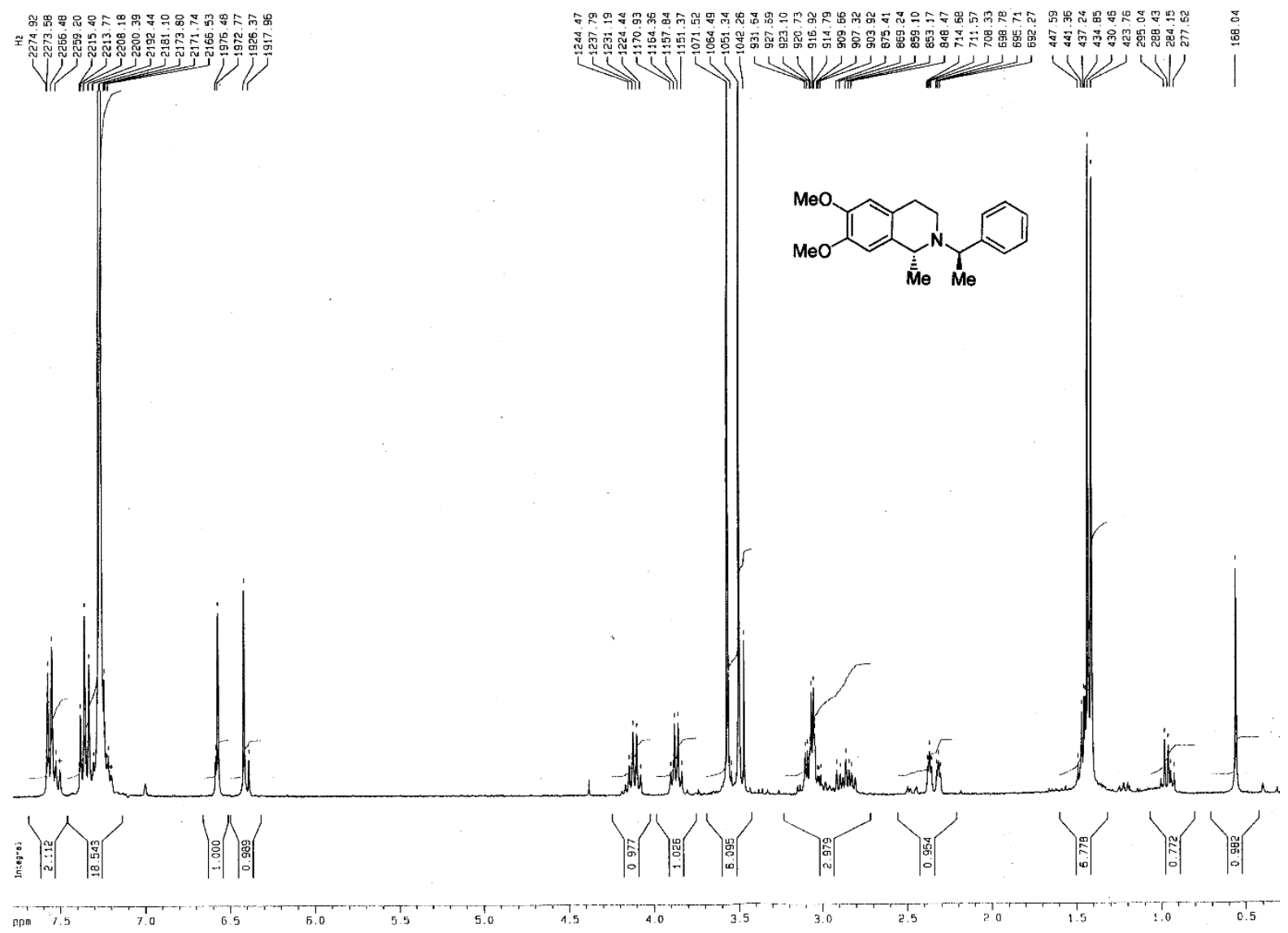

Figure S 69. ${ }^{1} \mathrm{H}$ NMR spectrum $\left(\mathrm{CDCl}_{3}, 300 \mathrm{MHz}\right)$ of derivative $(+)-1 \mathbf{k}$. 


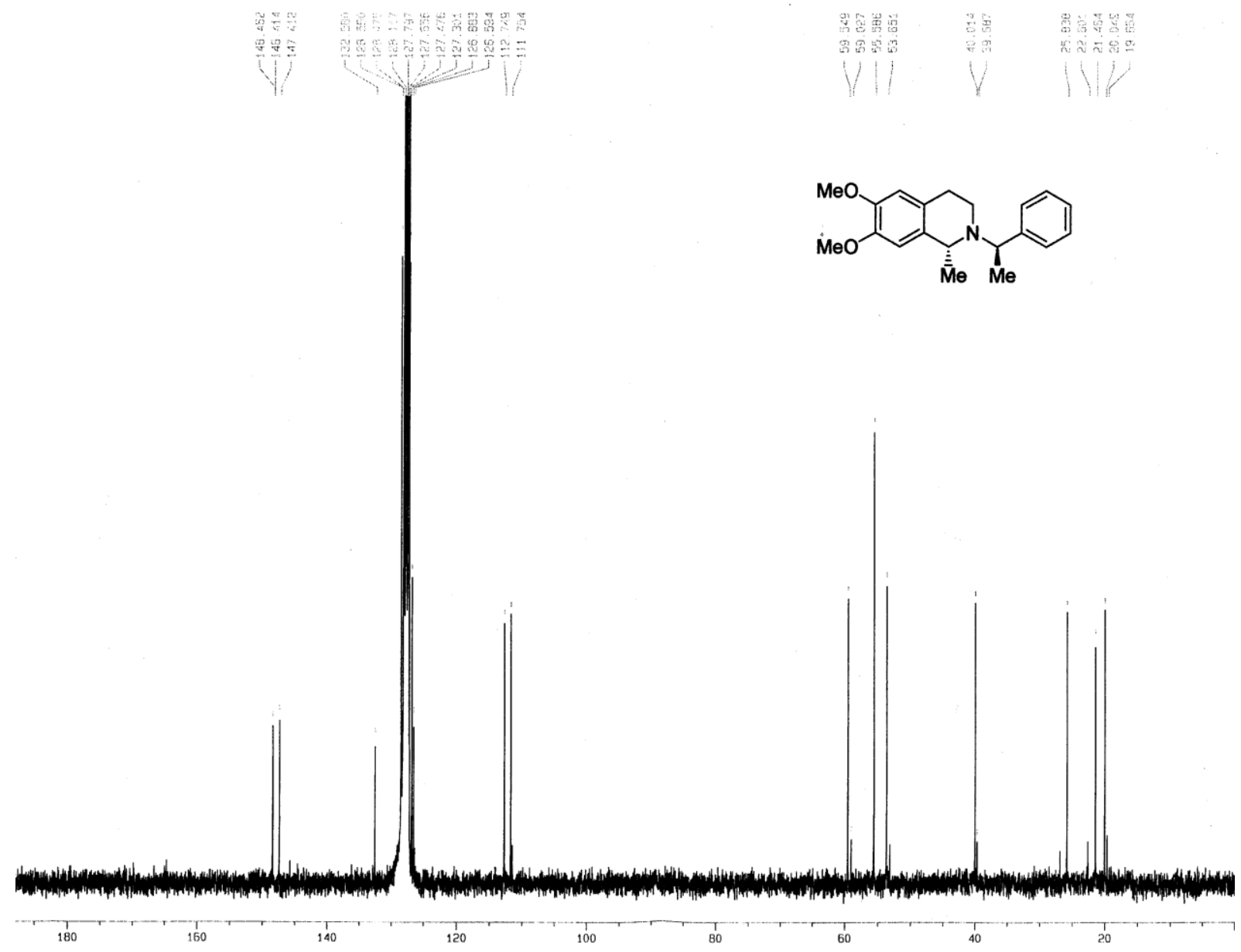

Figure S 70. ${ }^{13} \mathrm{C}$ NMR spectrum $\left(\mathrm{CDCl}_{3}, 75 \mathrm{MHz}\right)$ of derivative (+)-1k. 

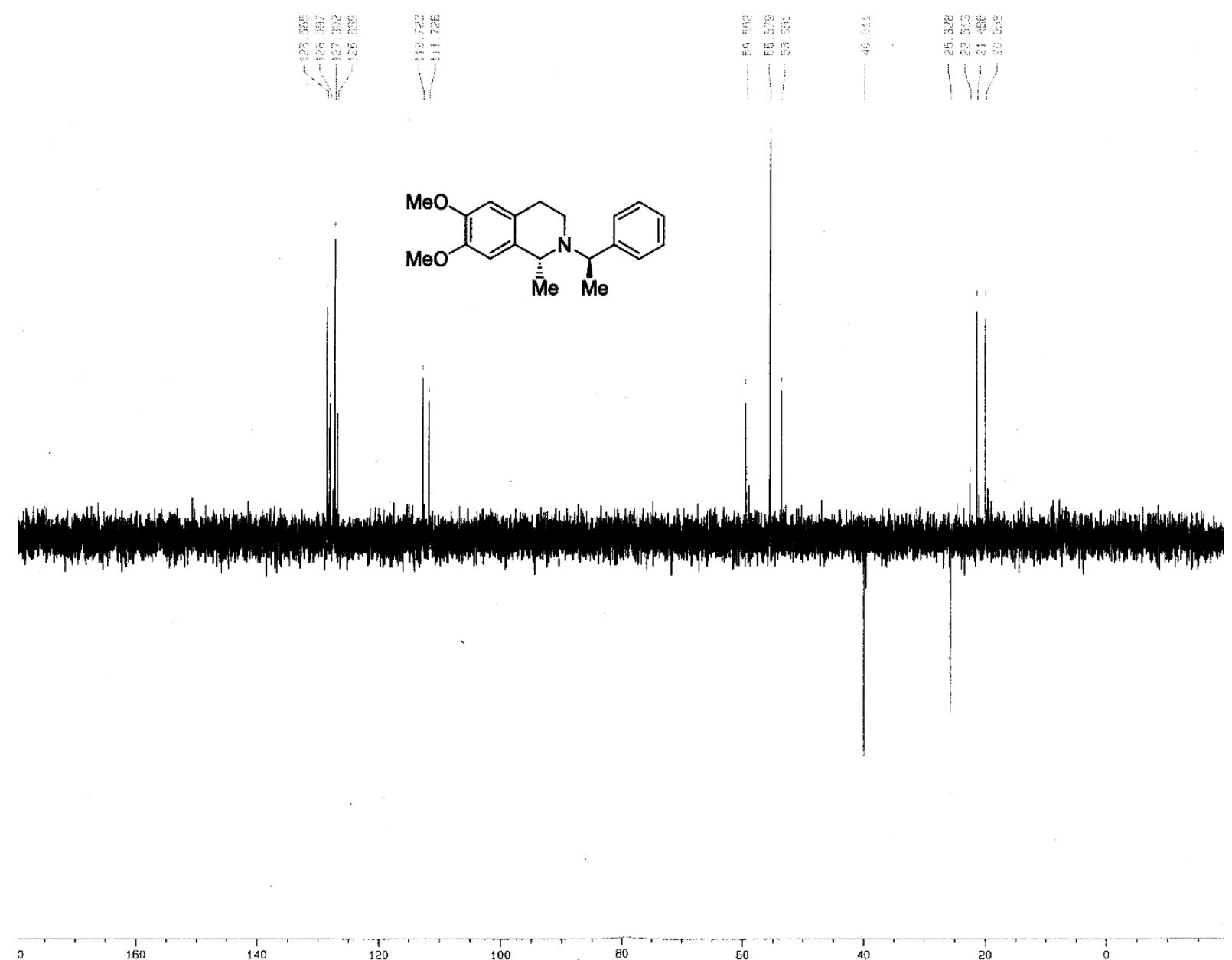

Figure S 71. DEPT C NMR spectrum $\left(\mathrm{CDCl}_{3}, 75 \mathrm{MHz}\right)$ of derivative (+)-1k. 


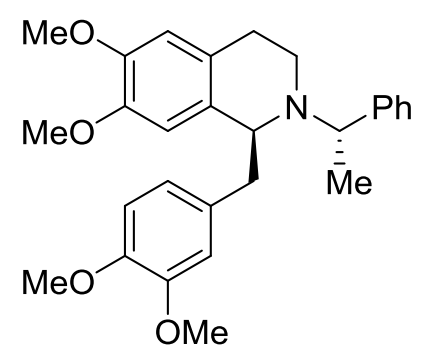

$(+)-11$

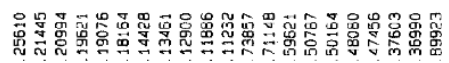

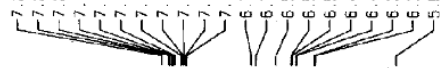

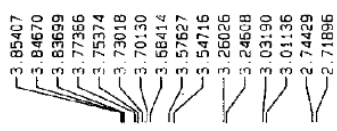

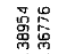

i

iั

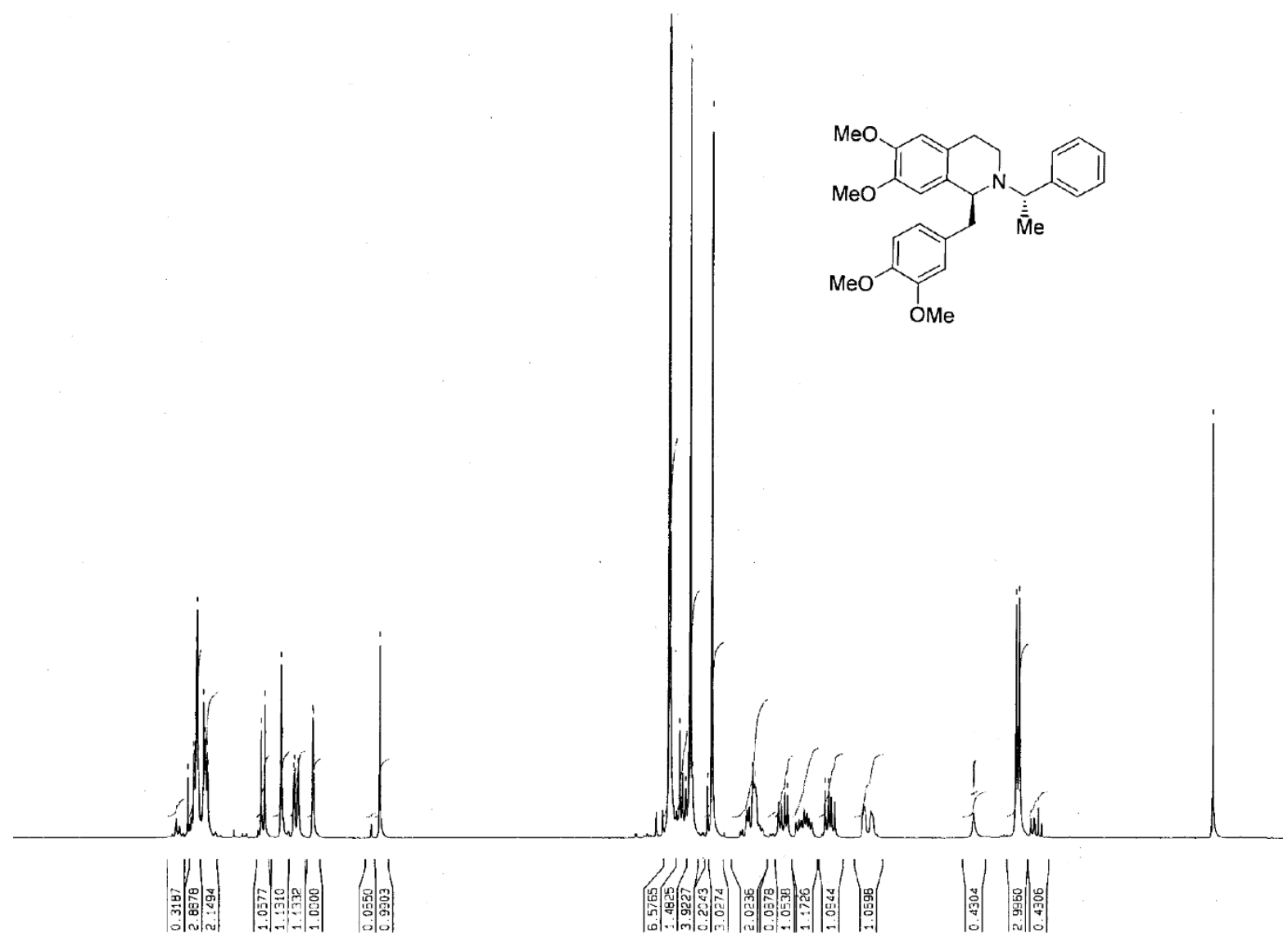

Figure S 72. ${ }^{1} \mathrm{H}$ NMR spectrum $\left(\mathrm{CDCl}_{3}, 400 \mathrm{MHz}\right)$ of derivative (+)-11. 


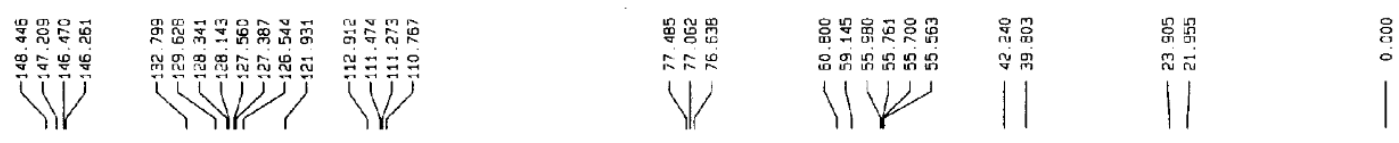

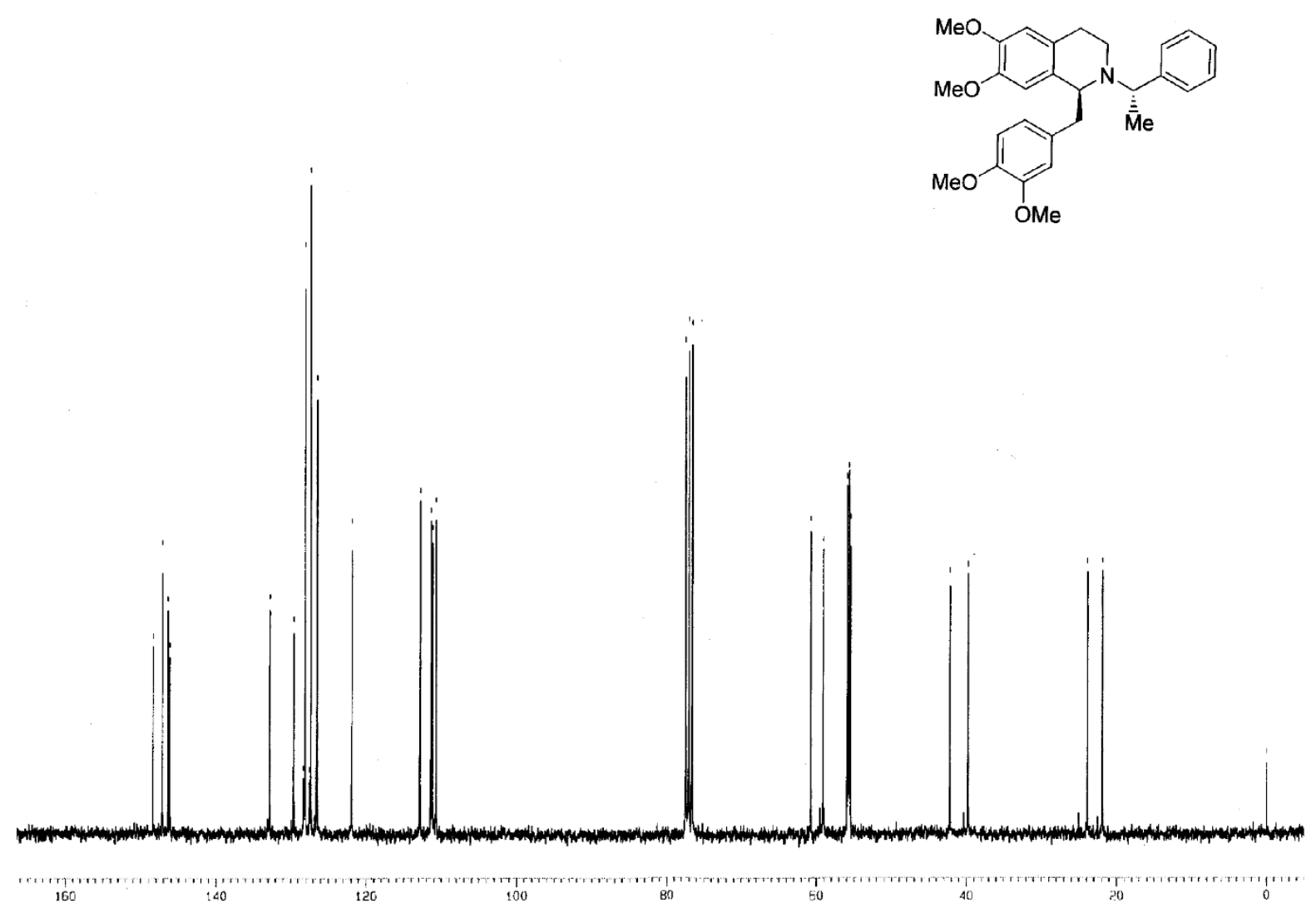

Figure S 73. ${ }^{13} \mathrm{C}$ NMR spectrum $\left(\mathrm{CDCl}_{3}, 100 \mathrm{MHz}\right)$ of derivative (+)-11. 


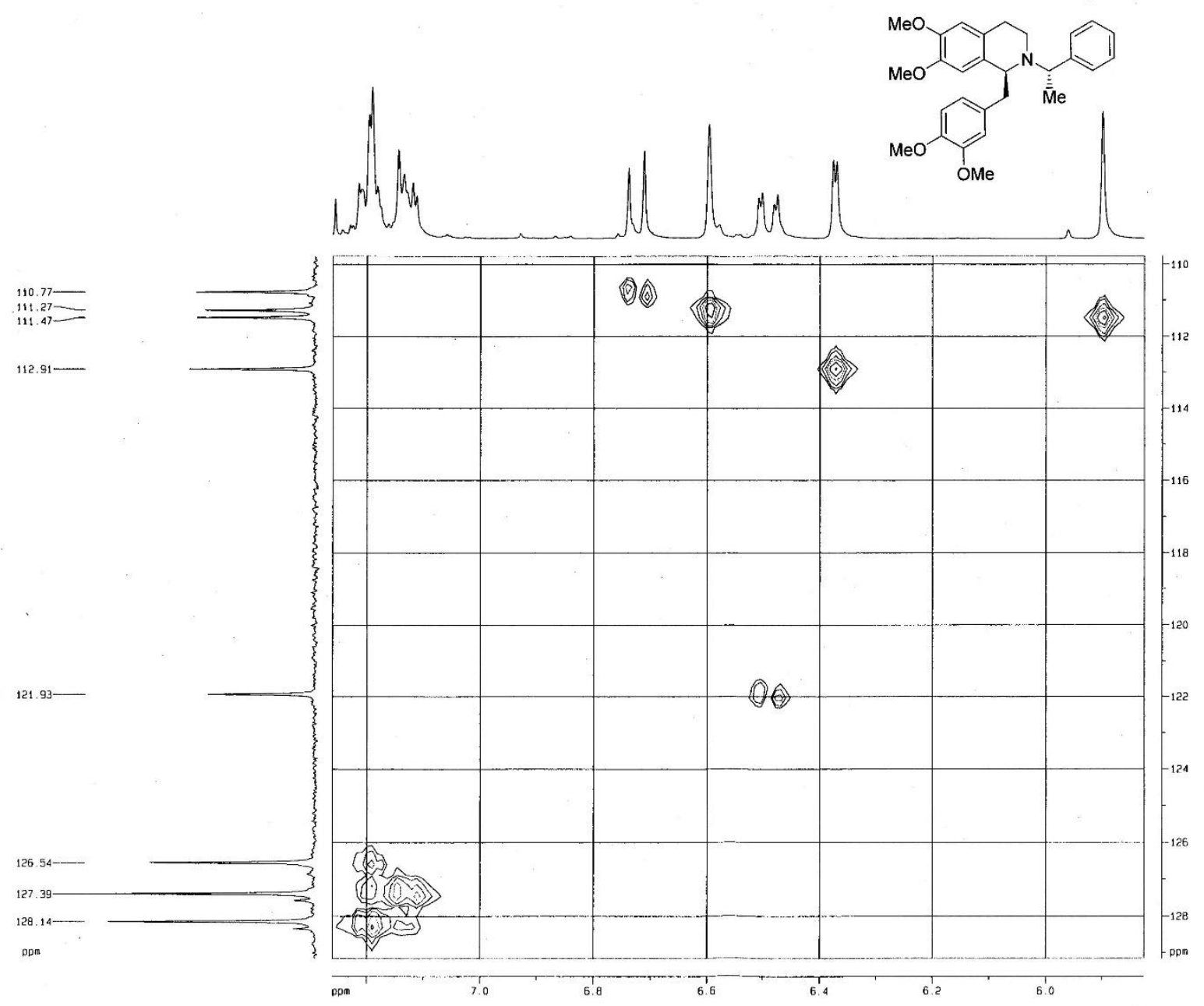

Figure $\mathbf{S}$ 74. $\mathrm{HMQC}$ spectrum $\left(\mathrm{CDCl}_{3}\right)$ of derivative $(+)-\mathbf{1 1}$. 


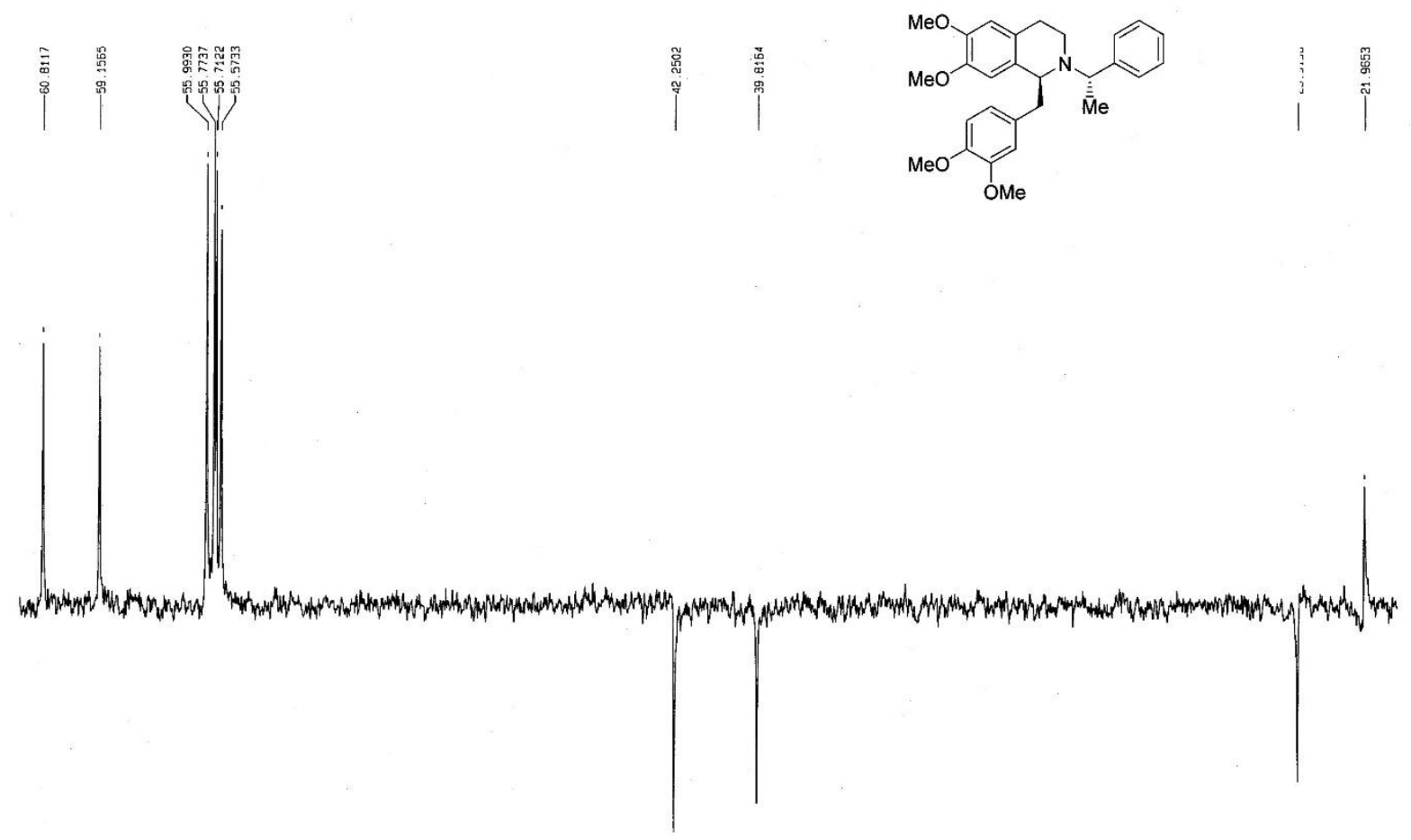

Figure S 75. DEPT C NMR spectrum $\left(\mathrm{CDCl}_{3}, 100 \mathrm{MHz}\right)$ of derivative (+)-11. 

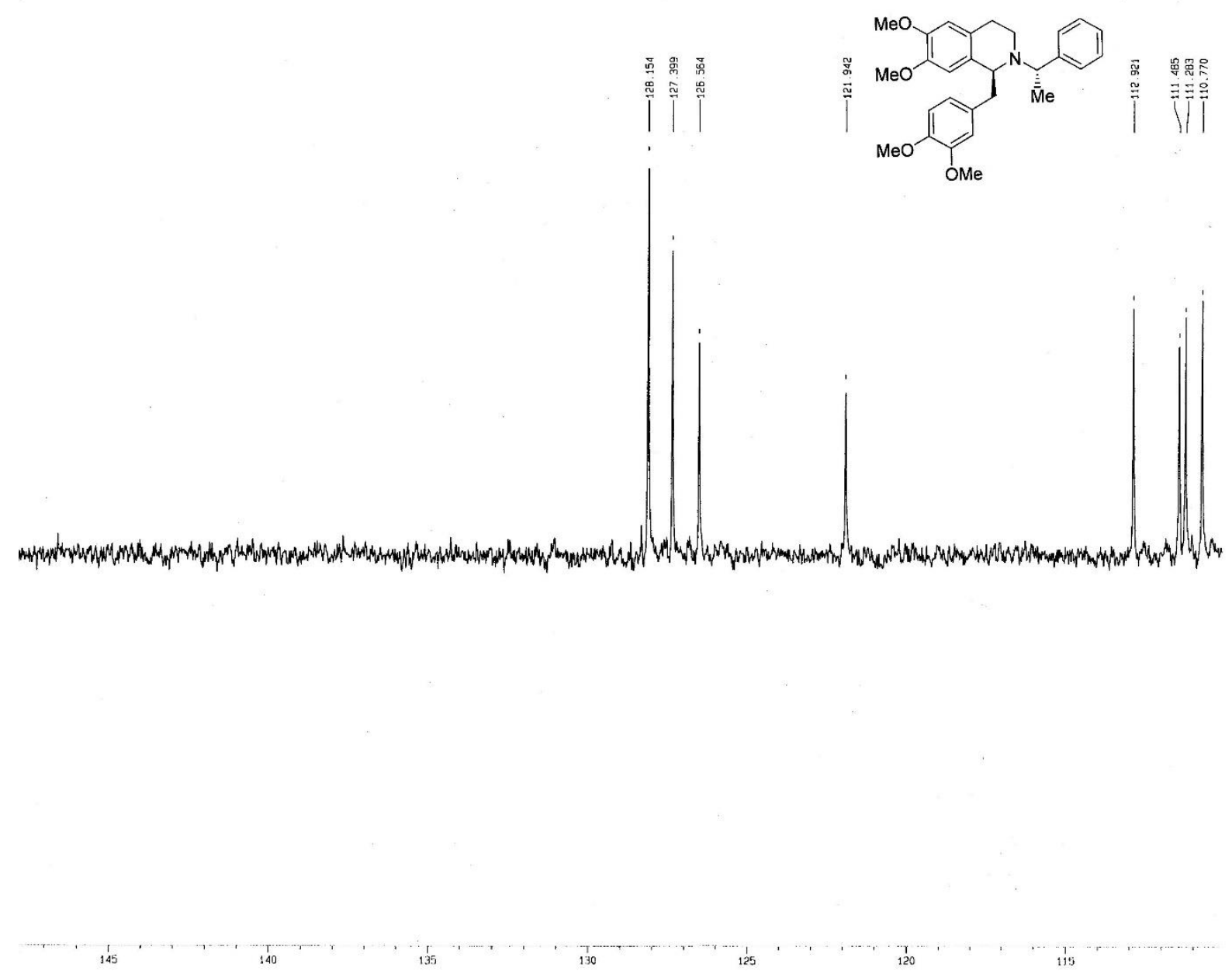

Figure S 76. DEPT C NMR spectrum $\left(\mathrm{CDCl}_{3}, 100 \mathrm{MHz}\right)$ of derivative (+)-11. 
<smiles>C[C@H]1NCCc2ccccc21</smiles>

$(-)-9 a$

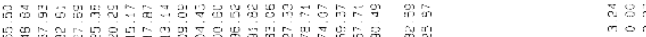

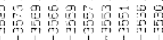

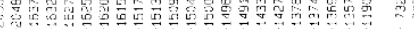
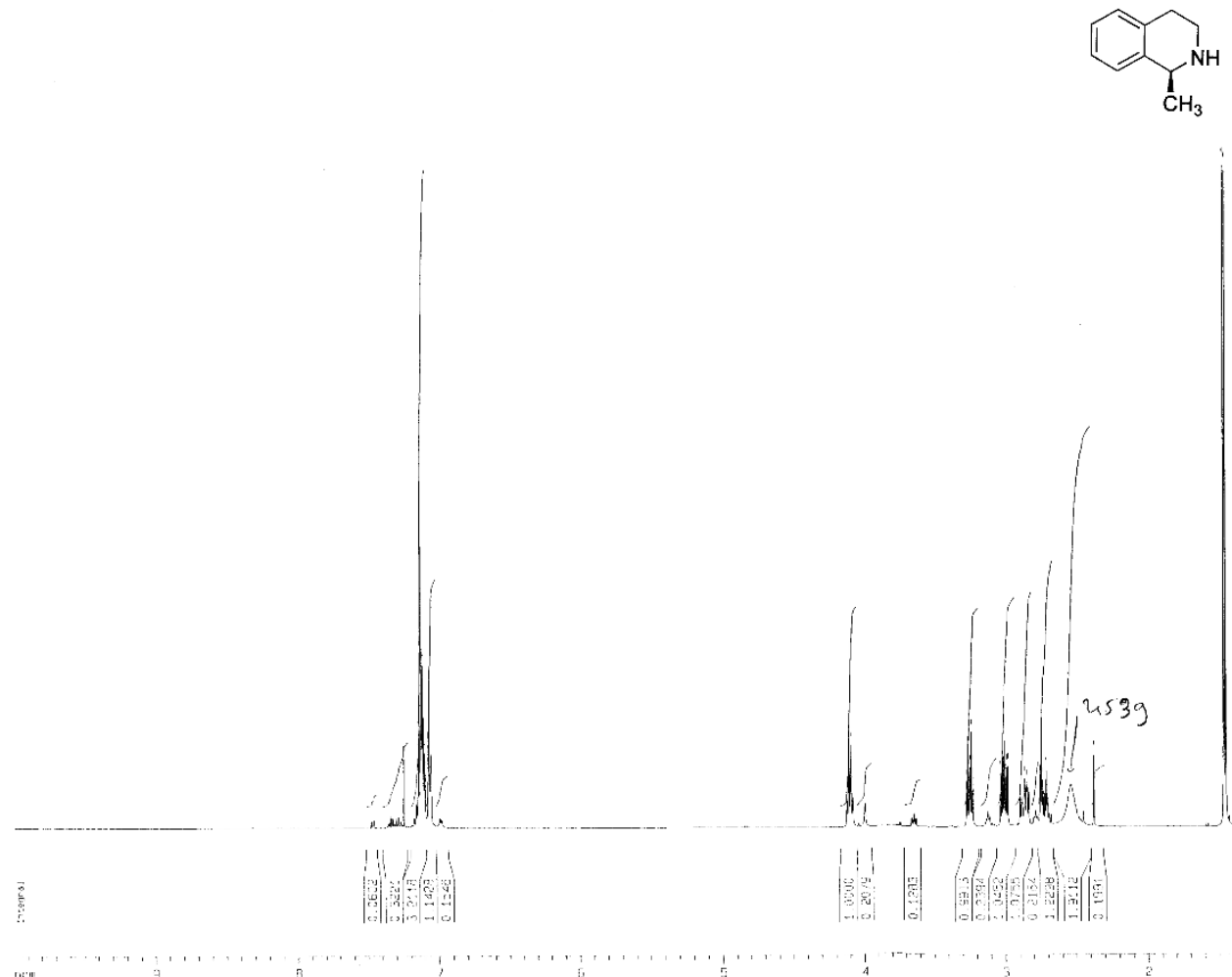

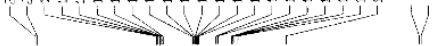

Figure S 77. ${ }^{1} \mathrm{H}$ NMR spectrum $\left(\mathrm{CDCl}_{3}, 500 \mathrm{MHz}\right)$ of derivative (-)-9a. 


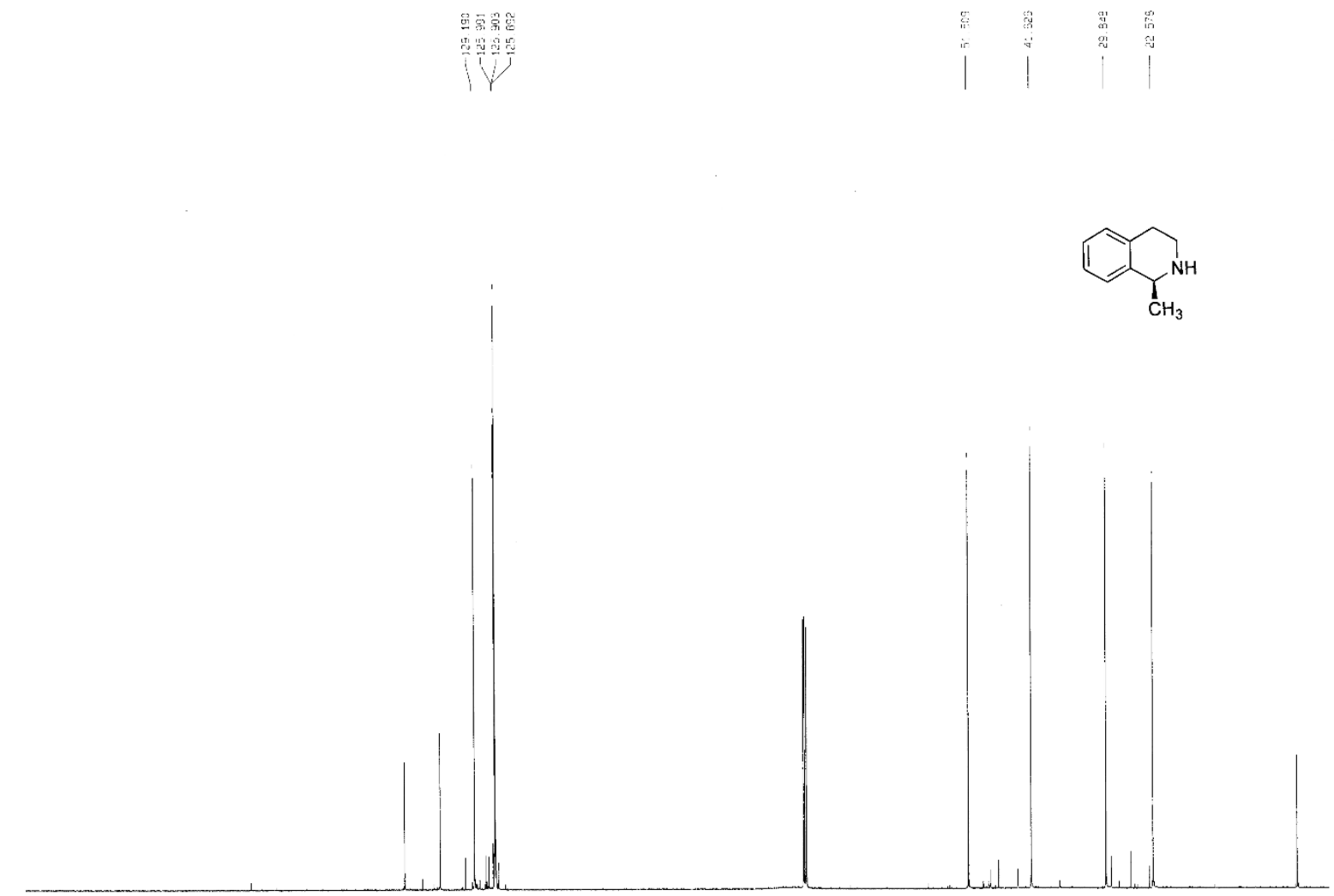

Figure S 78. ${ }^{13} \mathrm{C}$ NMR spectrum $\left(\mathrm{CDCl}_{3}, 125 \mathrm{MHz}\right)$ of derivative (-)-9a. 


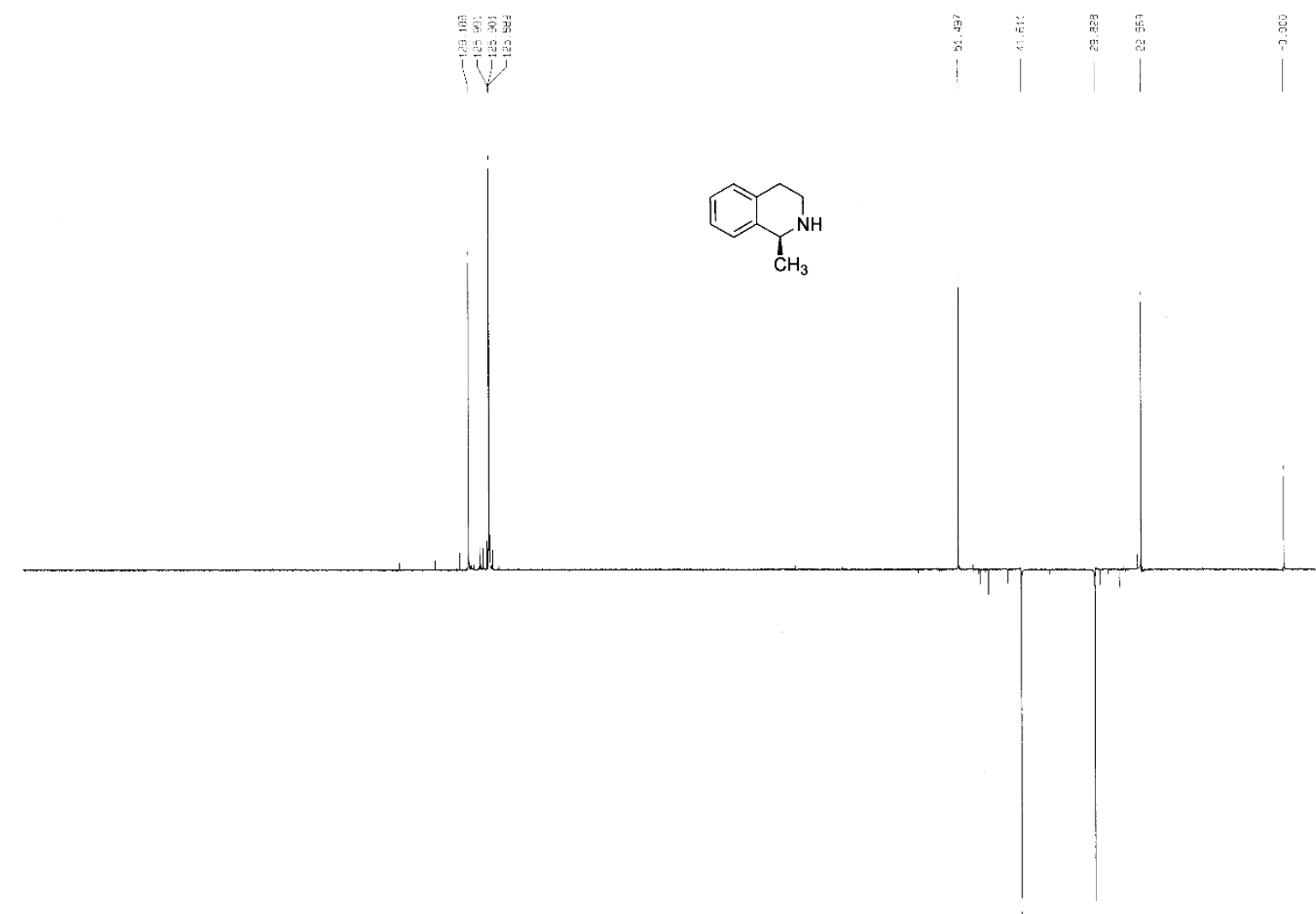

Figure S 79. DEPT C NMR spectrum ( $\left.\mathrm{CDCl}_{3}, 100 \mathrm{MHz}\right)$ of derivative (-)-9a. 


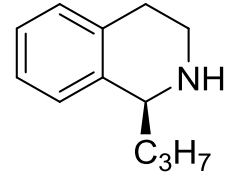

$(-)-9 b$

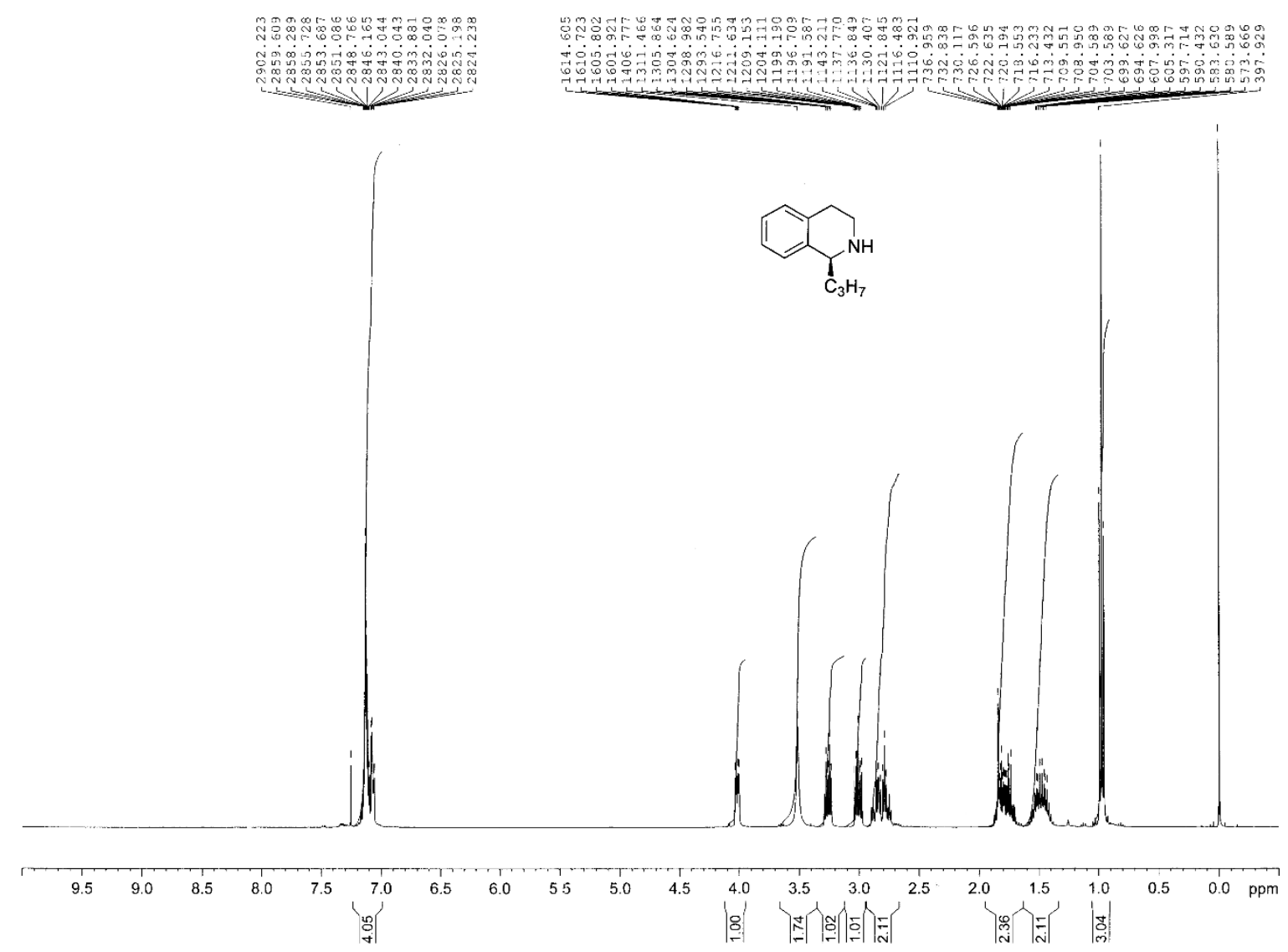

Figure S 80. ${ }^{1} \mathrm{H}$ NMR spectrum $\left(\mathrm{CDCl}_{3}, 400 \mathrm{MHz}\right)$ of derivative (-)-9b. 


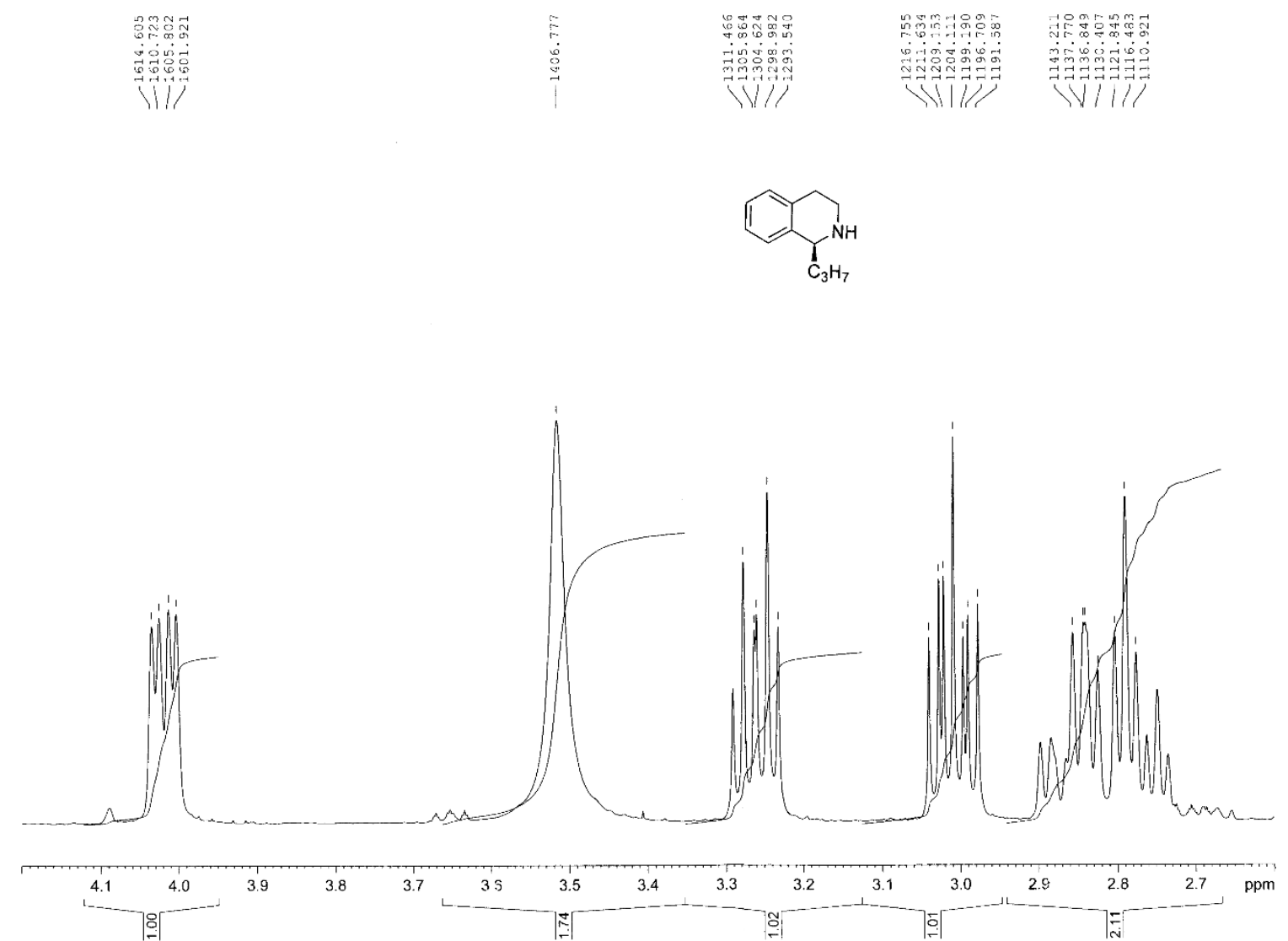

Figure S 81. ${ }^{1} \mathrm{H} \mathrm{NMR}$ spectrum $\left(\mathrm{CDCl}_{3}, 400 \mathrm{MHz}\right)$ of derivative (-)-9b. 


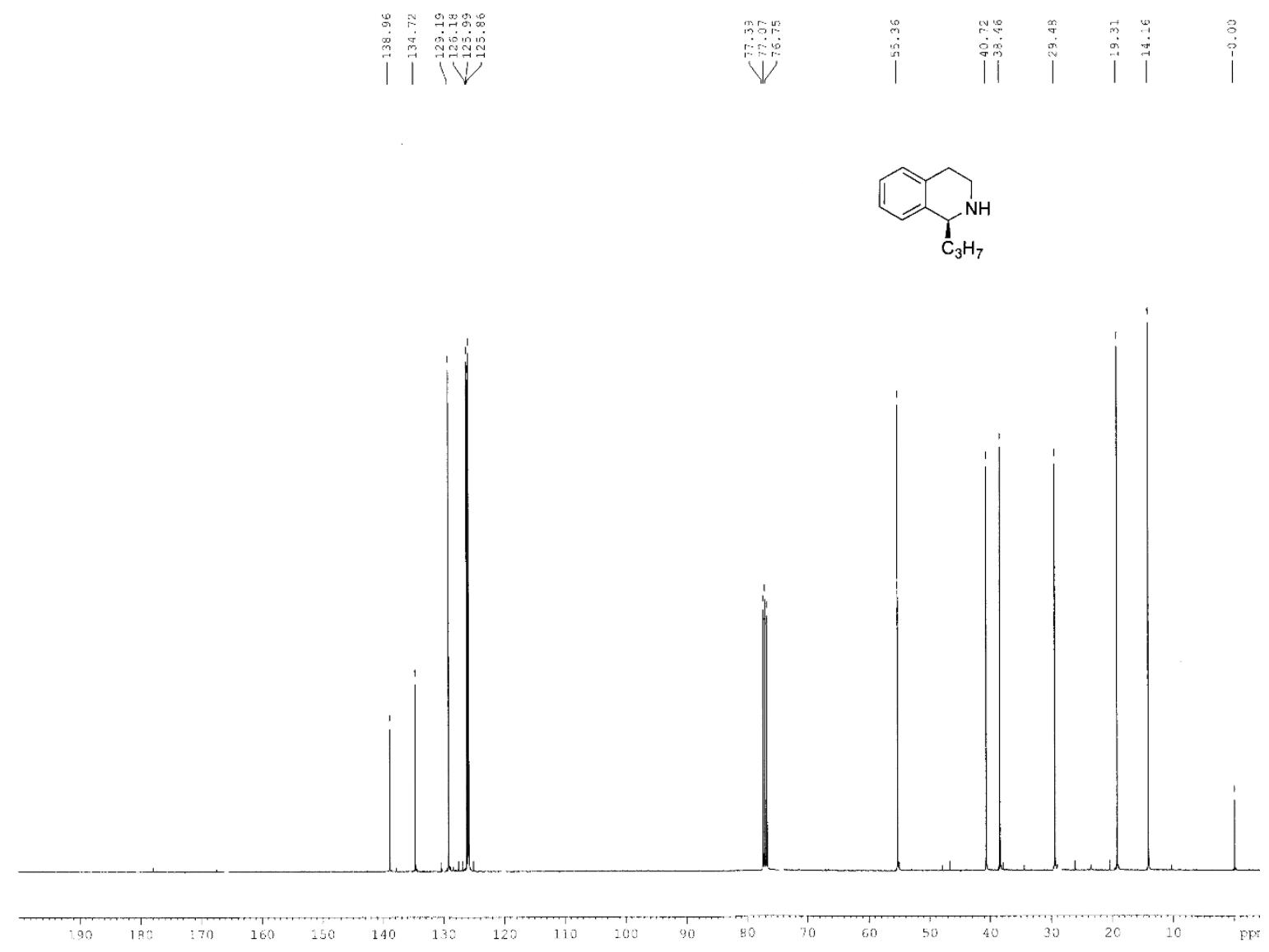

Figure S 82. ${ }^{13} \mathrm{C}$ NMR spectrum $\left(\mathrm{CDCl}_{3}, 100 \mathrm{MHz}\right)$ of derivative (-)-9b. 


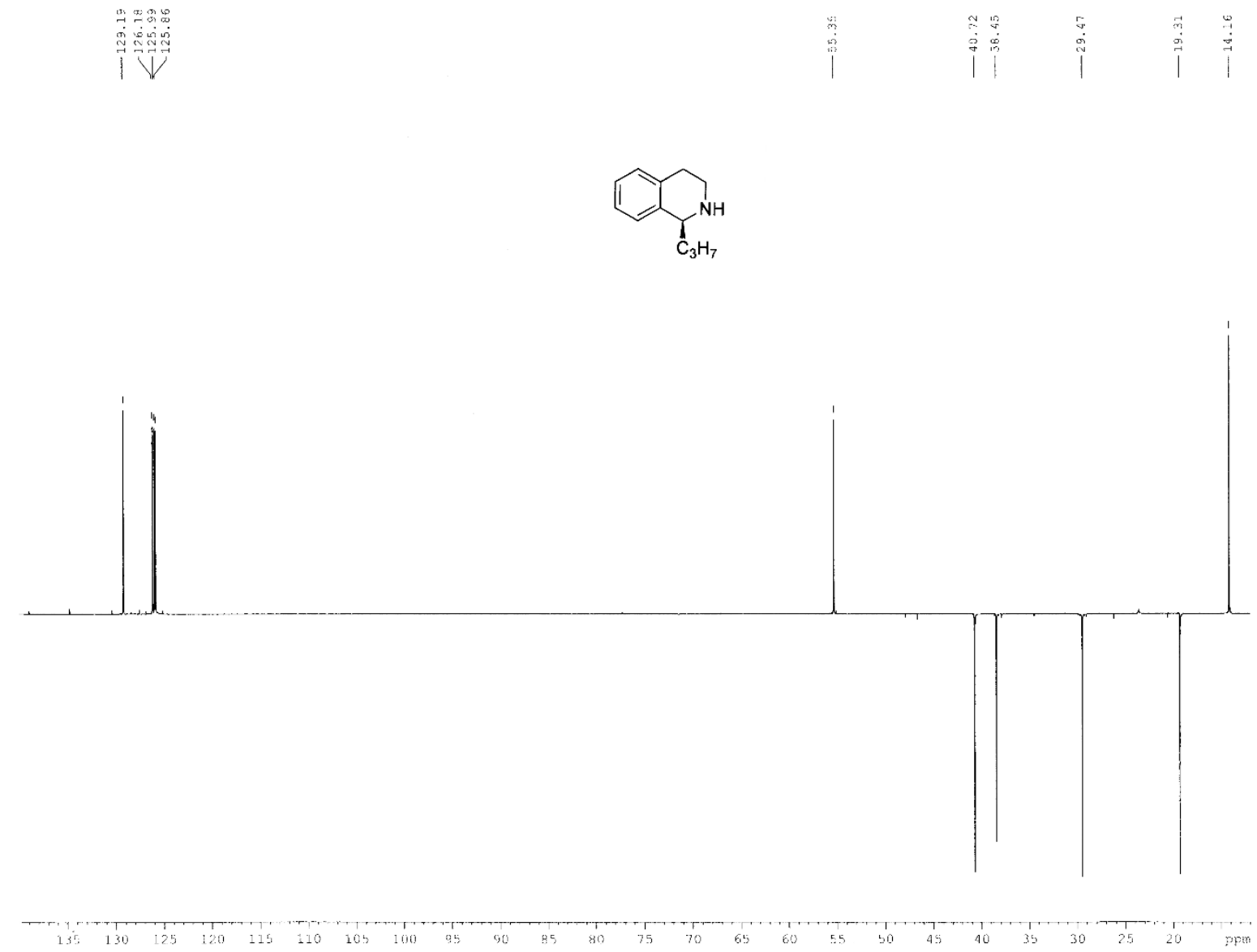

Figure S 83. DEPT C-NMR spectrum ( $\left.\mathrm{CDCl}_{3}, 100 \mathrm{MHz}\right)$ of derivative (-)-9b. 
<smiles>[13CH3][C@@H]1NCCc2ccccc21</smiles>

$(-)-9 c$

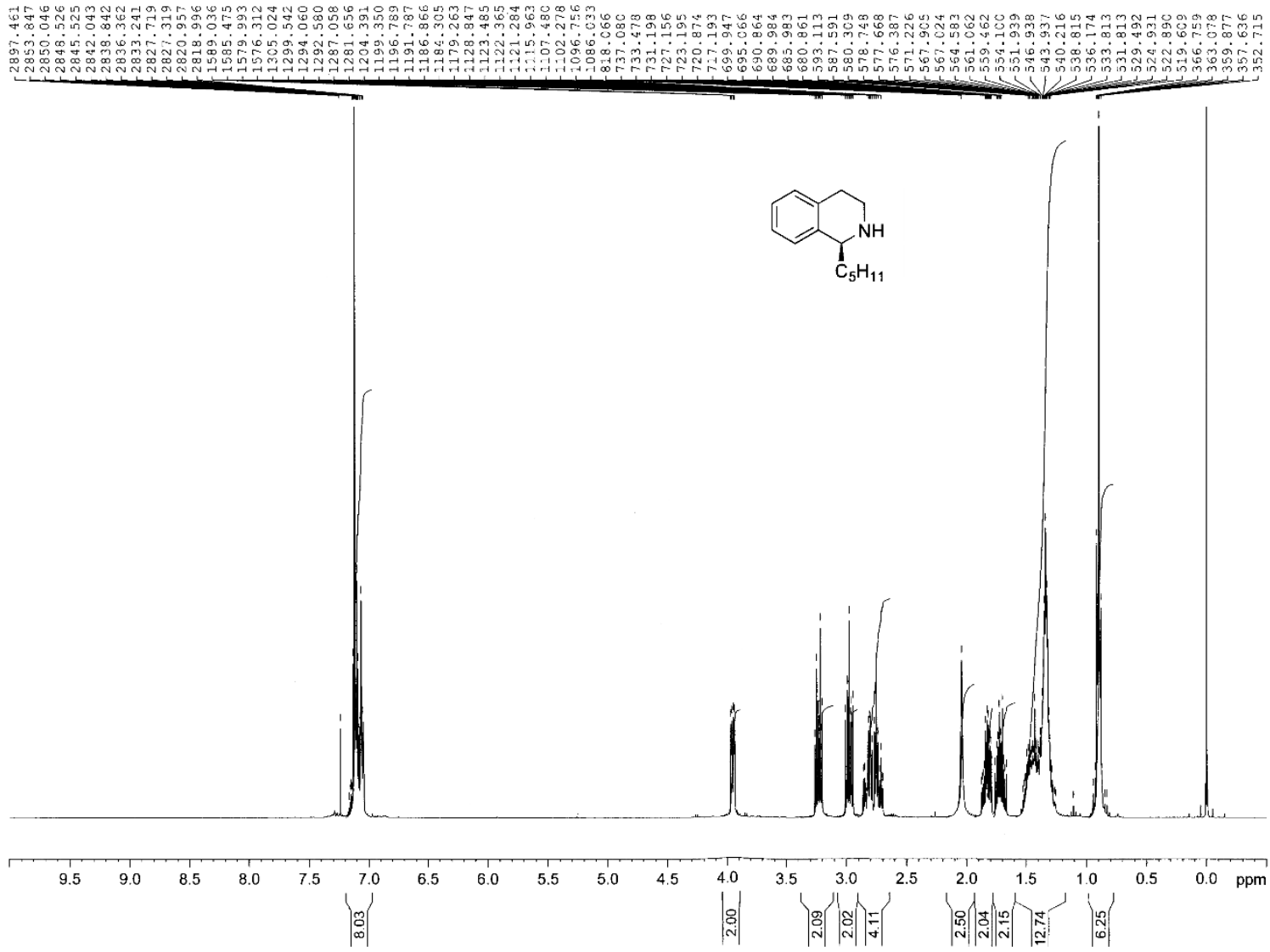

Figure S 84. ${ }^{1} \mathrm{H}$ NMR spectrum $\left(\mathrm{CDCl}_{3}, 400 \mathrm{MHz}\right)$ of derivative (-)-9c. 


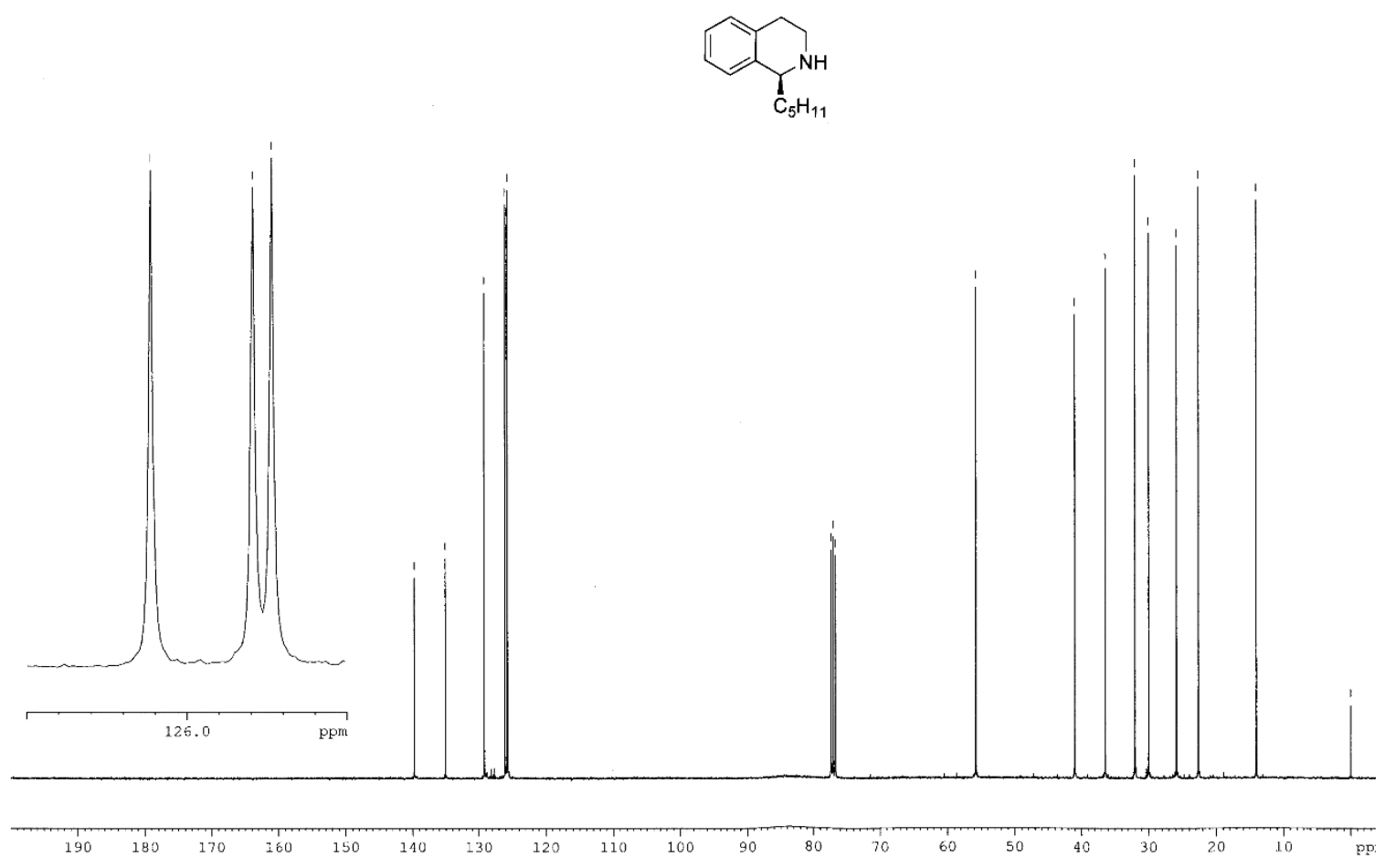

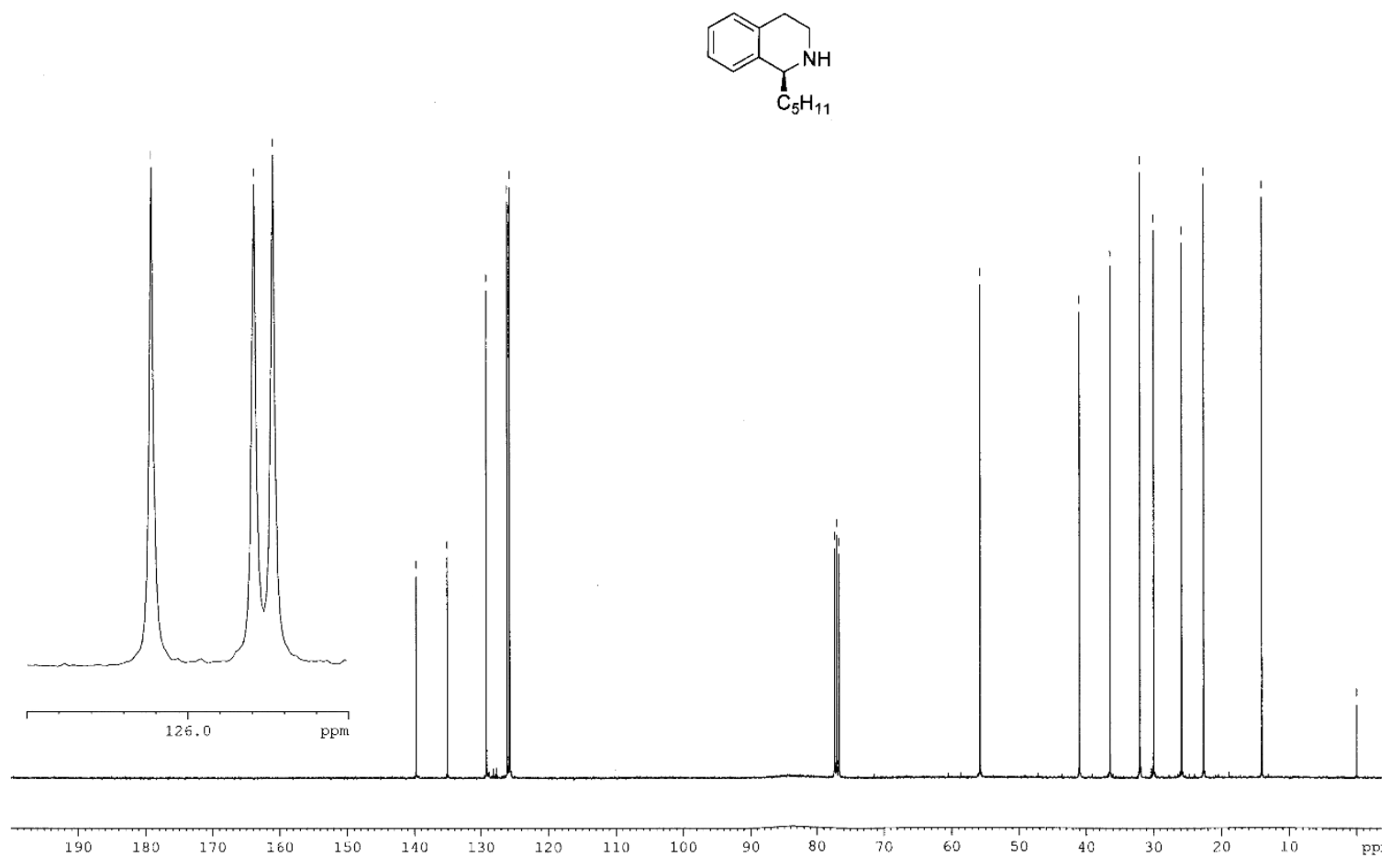

Figure S 85. ${ }^{13} \mathrm{C}$ NMR spectrum $\left(\mathrm{CDCl}_{3}, 100 \mathrm{MHz}\right)$ of derivative (-)-9c. 
S88

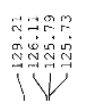
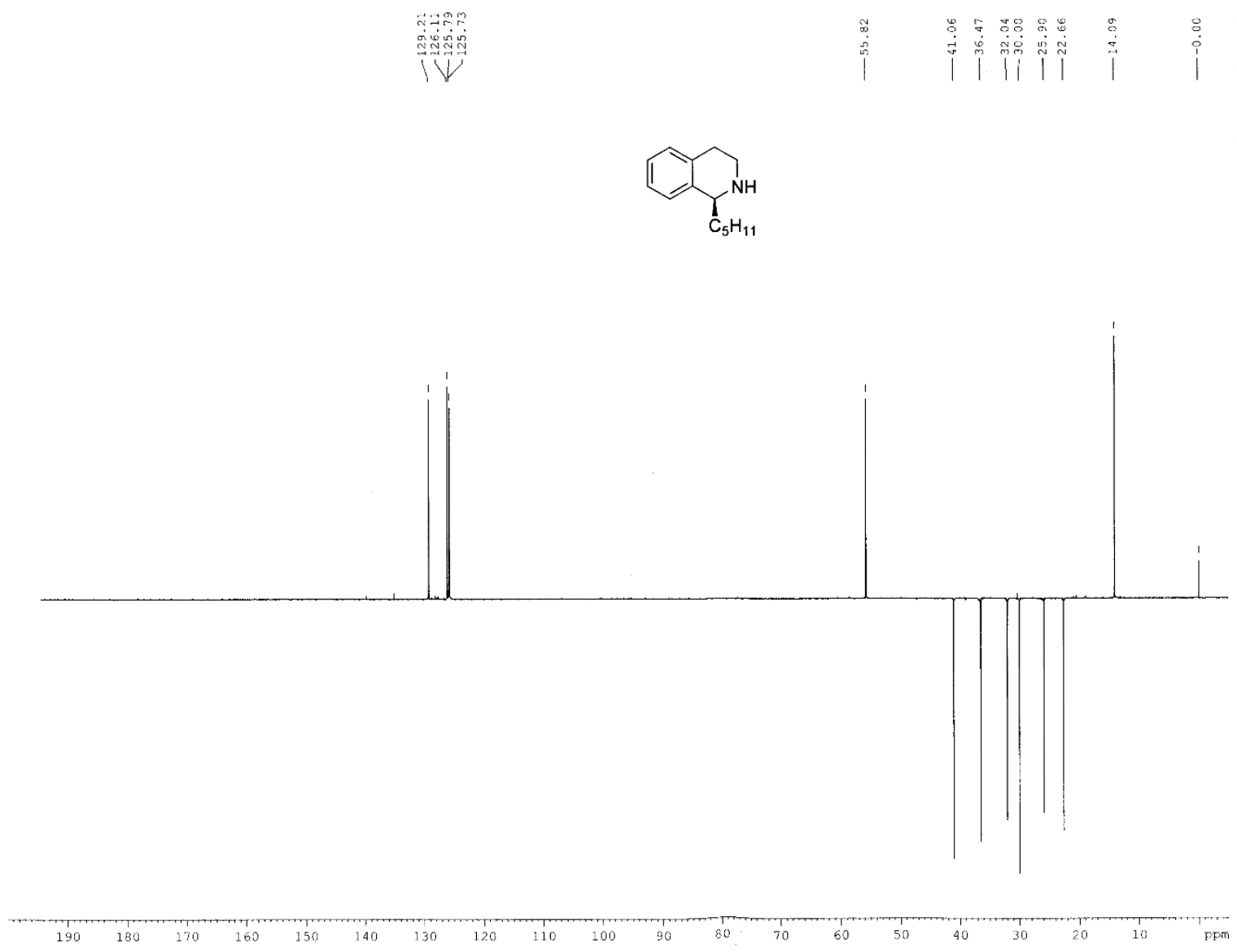

Figure $\mathbf{S}$ 86. DEPT C NMR spectrum $\left(\mathrm{CDCl}_{3}, 100 \mathrm{MHz}\right)$ of derivative (-)-qc. 


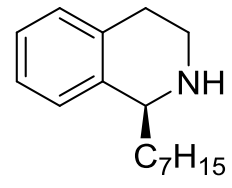

$(-)-9 d$

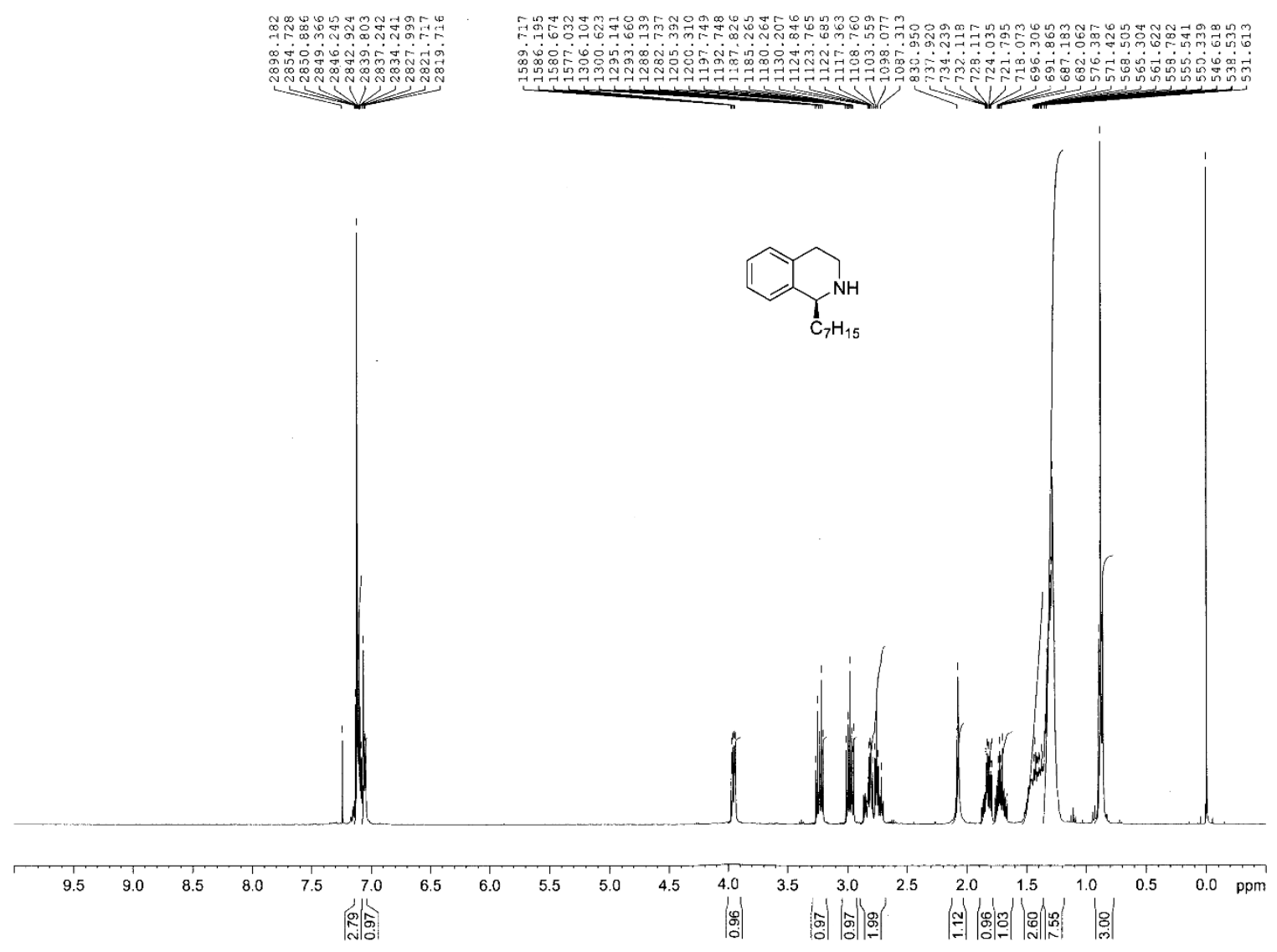

Figure S 87. ${ }^{1} \mathrm{H}$ NMR spectrum $\left(\mathrm{CDCl}_{3}, 400 \mathrm{MHz}\right)$ of derivative (-)-9d. 

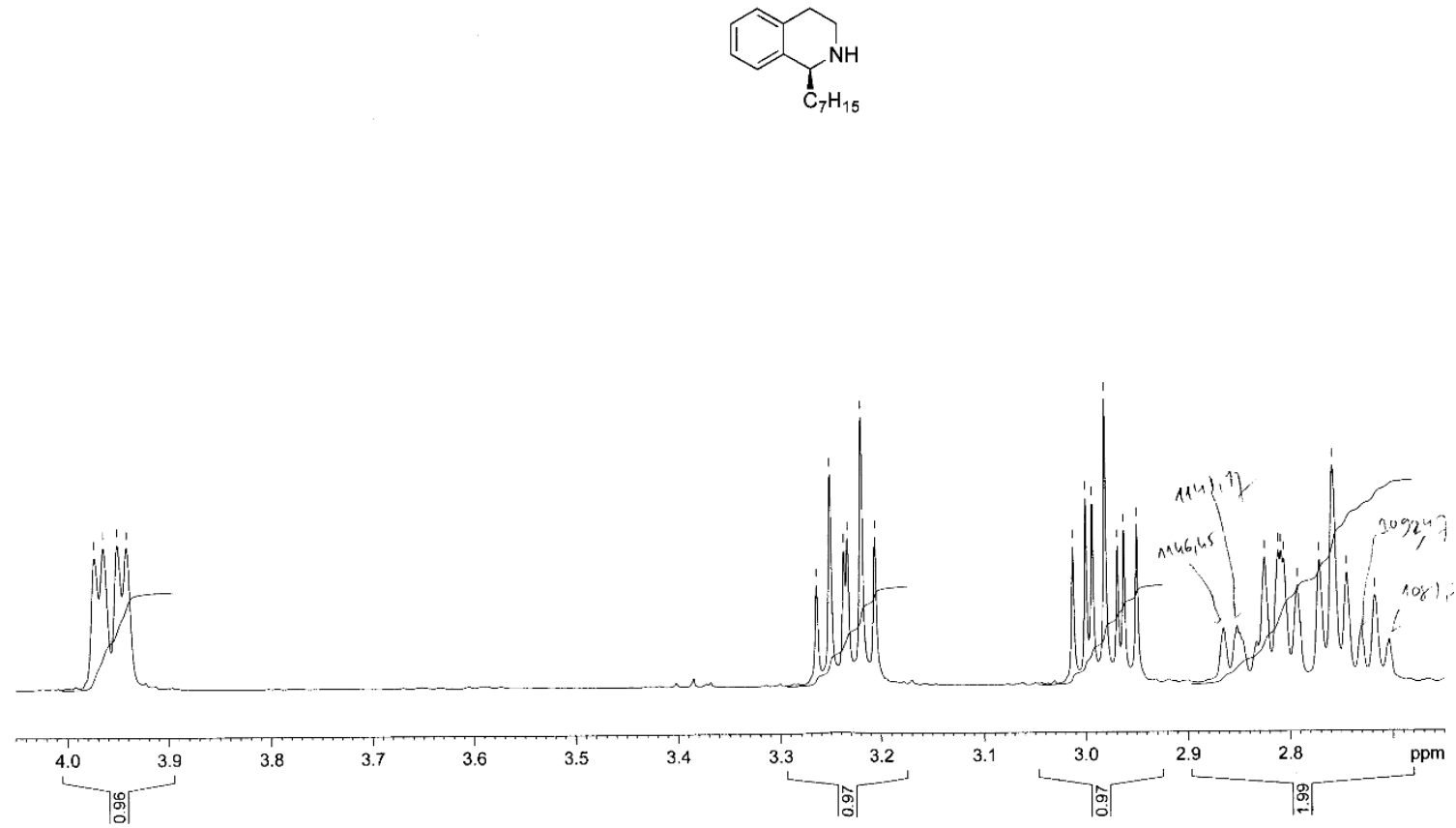

Figure S 88. ${ }^{1} \mathrm{H}$ NMR spectrum $\left(\mathrm{CDCl}_{3}, 400 \mathrm{MHz}\right)$ of derivative (-)-9d. 


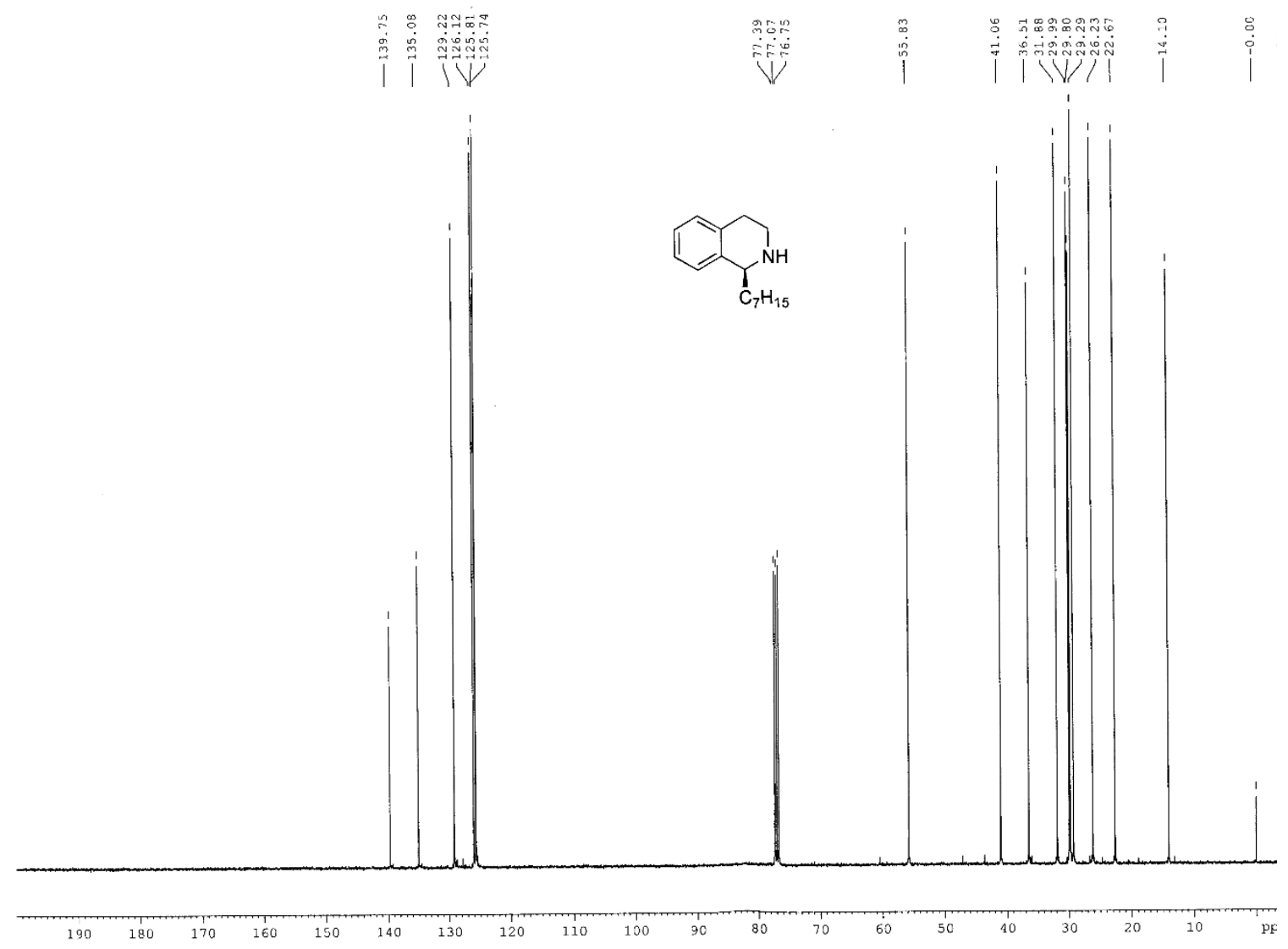

Figure S 89. ${ }^{13} \mathrm{C}$ NMR spectrum $\left(\mathrm{CDCl}_{3}, 100 \mathrm{MHz}\right)$ of derivative (-)-9d. 


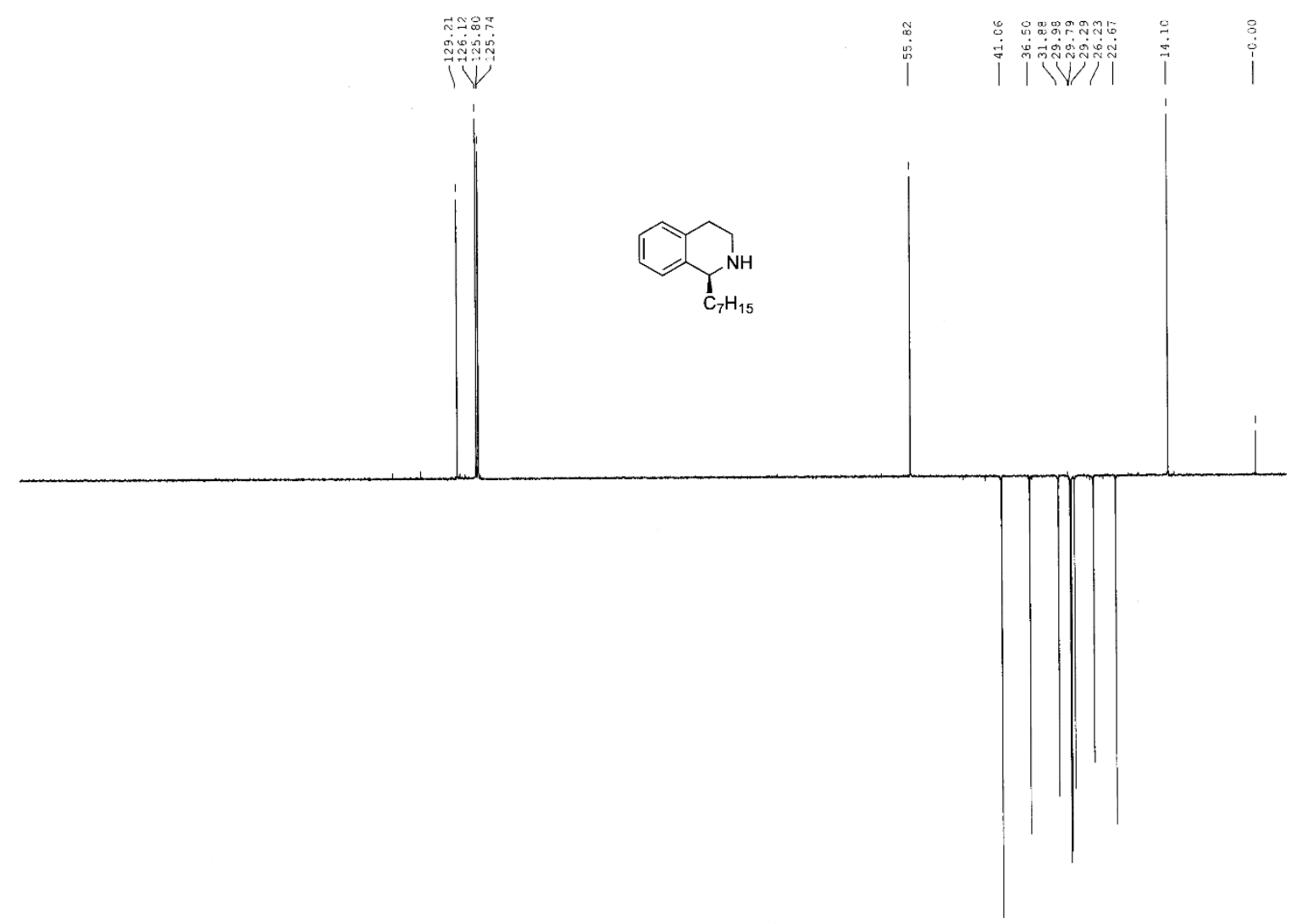

Figure S 90. DEPT C NMR spectrum ( $\left.\mathrm{CDCl}_{3}, 100 \mathrm{MHz}\right)$ of derivative (-)-9d. 


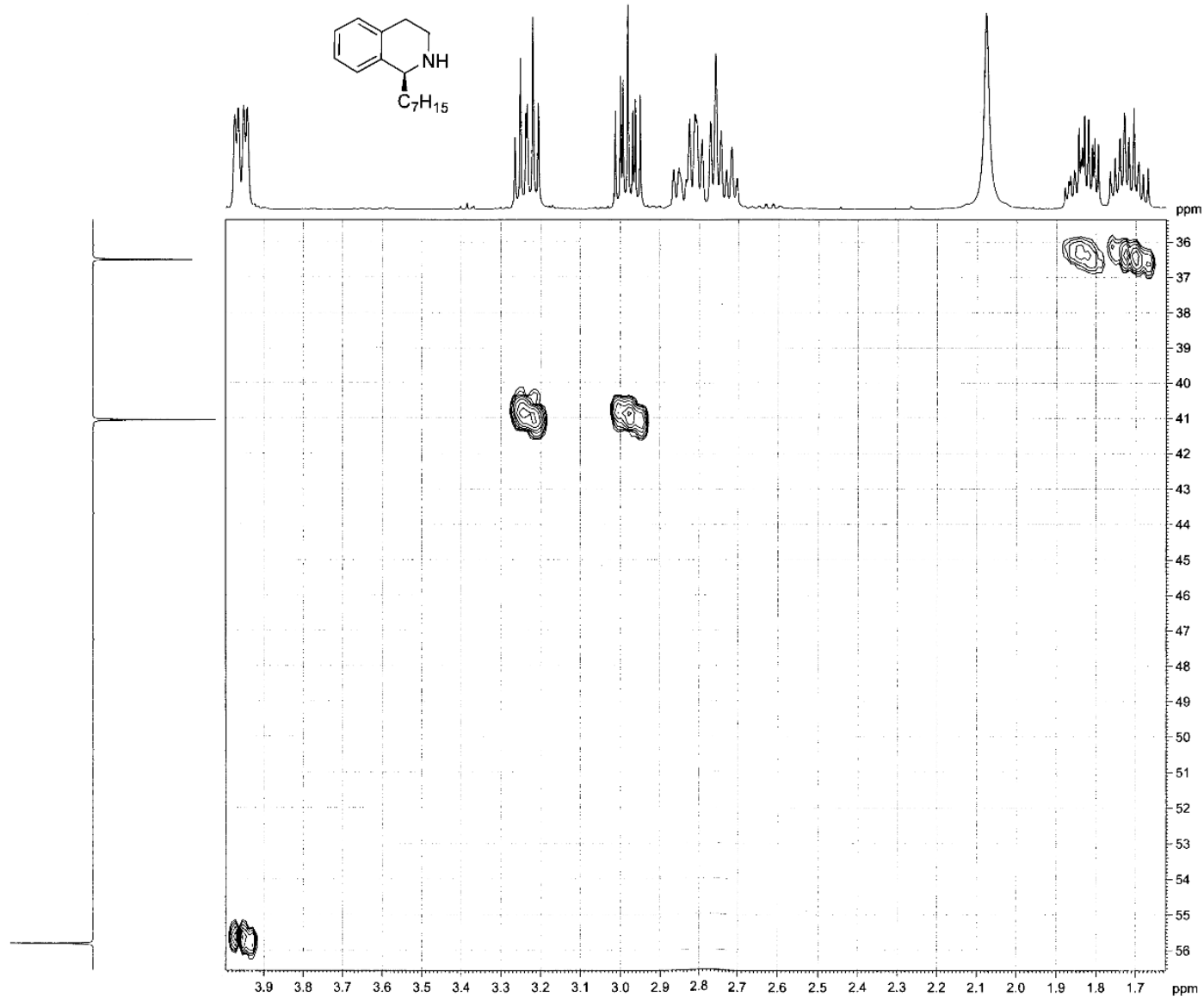

Figure S 91. HMQC spectrum $\left(\mathrm{CDCl}_{3}\right)$ of derivative (-)-9d. 


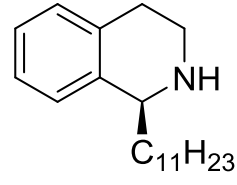

$(-)-9 e$

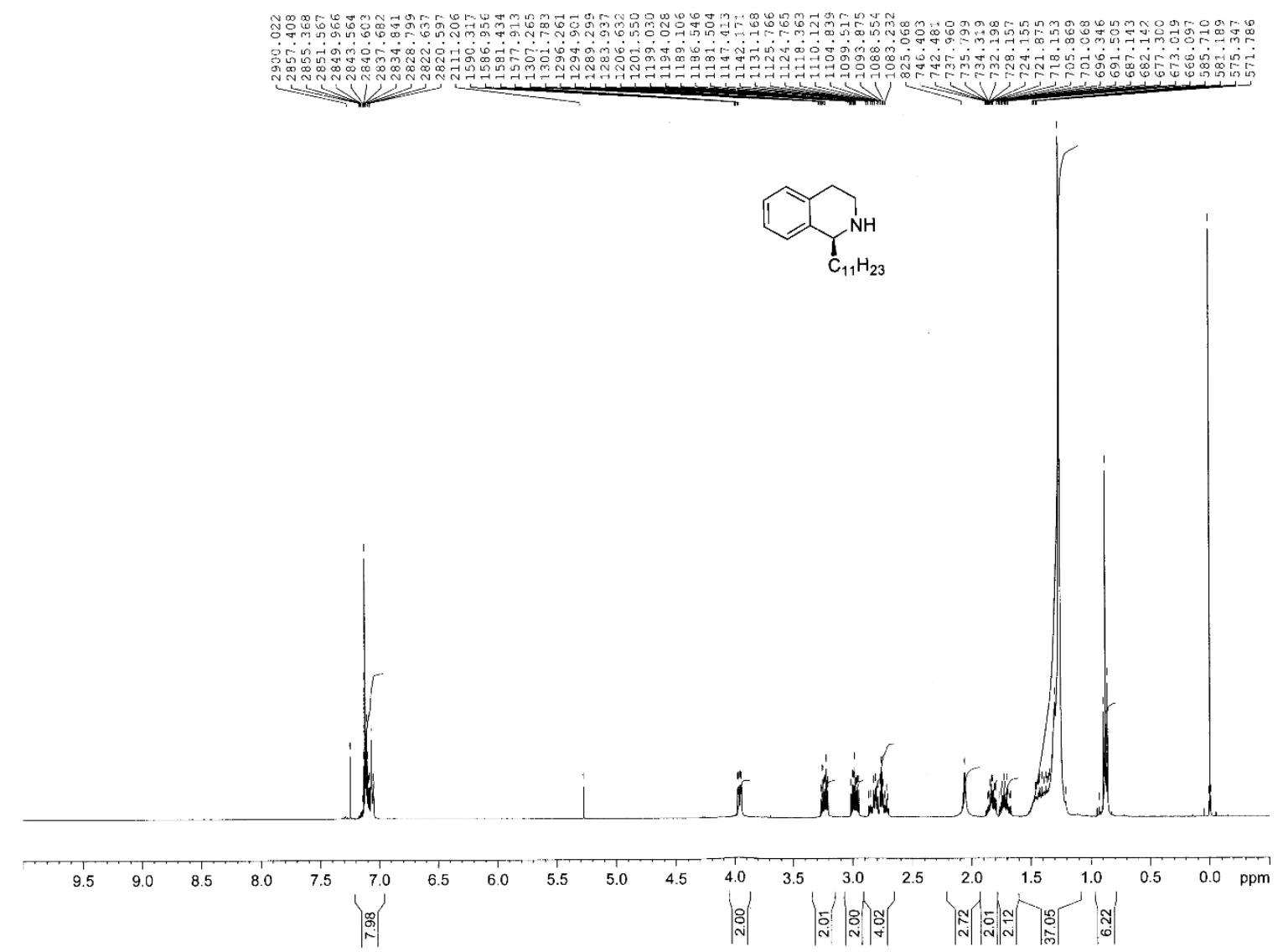

Figure S 92. ${ }^{1} \mathrm{H} \mathrm{NMR}$ spectrum $\left(\mathrm{CDCl}_{3}, 400 \mathrm{MHz}\right)$ of derivative (-)-9e. 


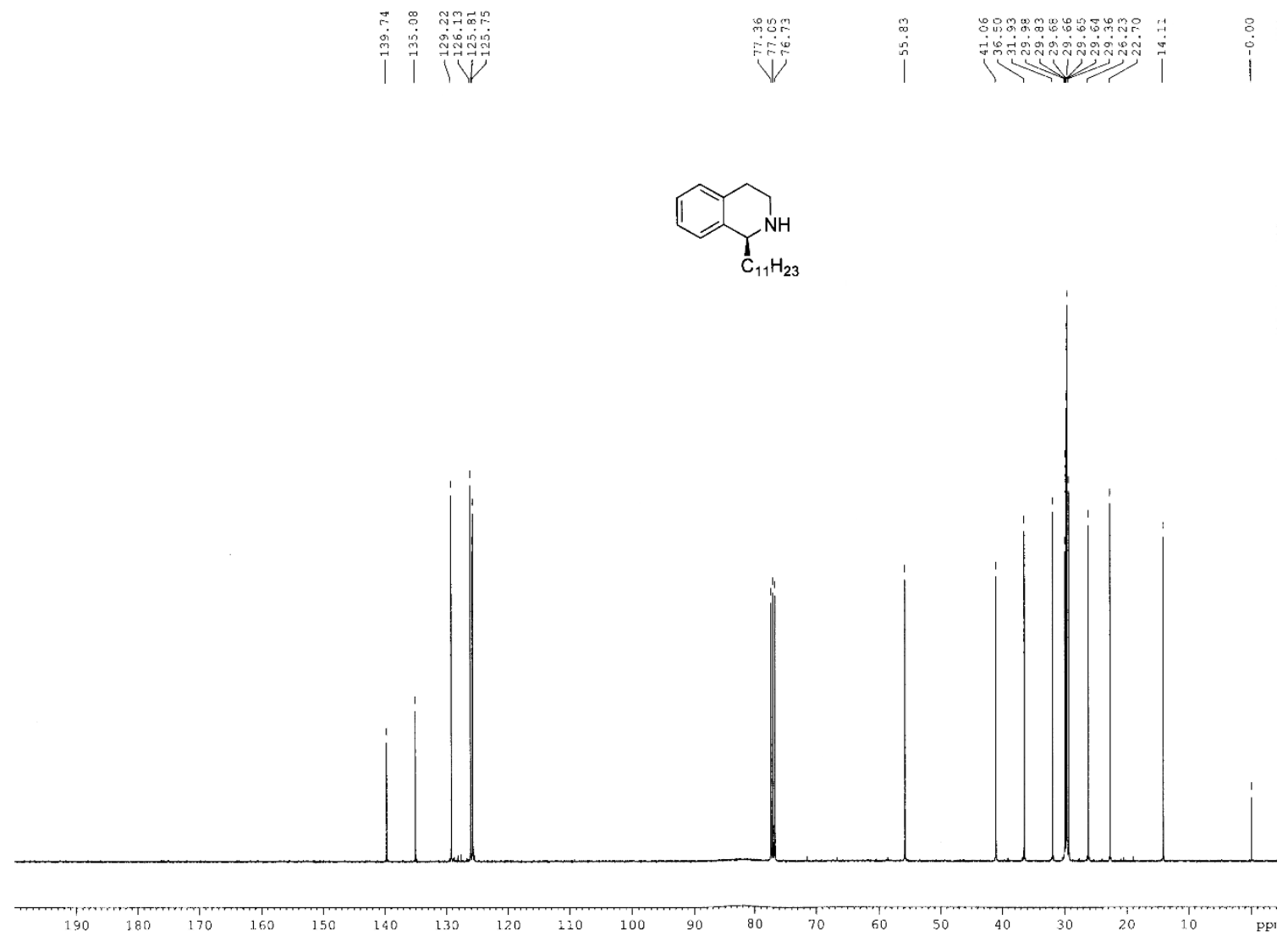

Figure S 93. ${ }^{13} \mathrm{C}-\mathrm{NMR}$ spectrum $\left(\mathrm{CDCl}_{3}, 400 \mathrm{MHz}\right)$ of derivative (-)-9e. 


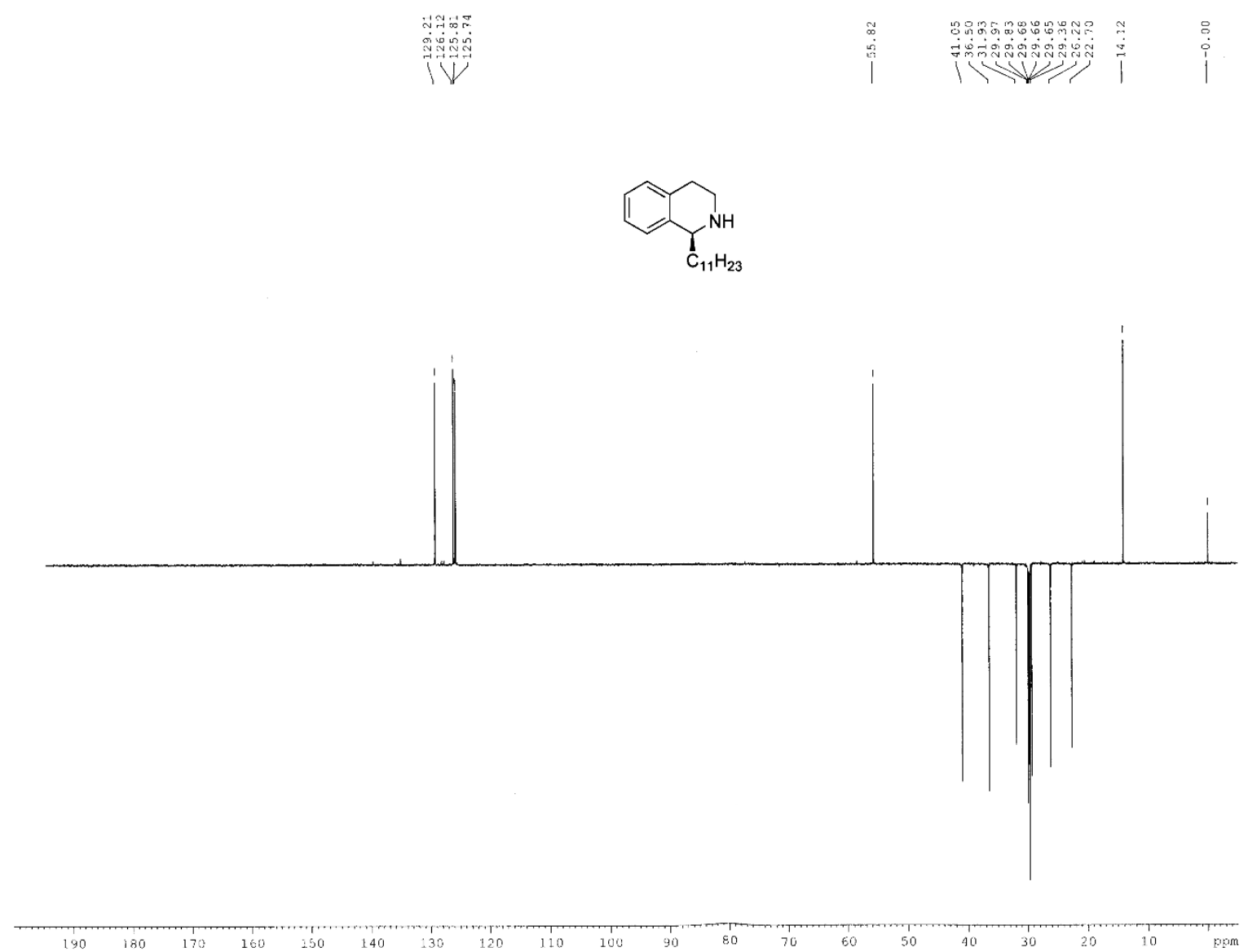

Figure S 94. DEPT C NMR spectrum ( $\left.\mathrm{CDCl}_{3}, 100 \mathrm{MHz}\right)$ of derivative (-)-9e. 


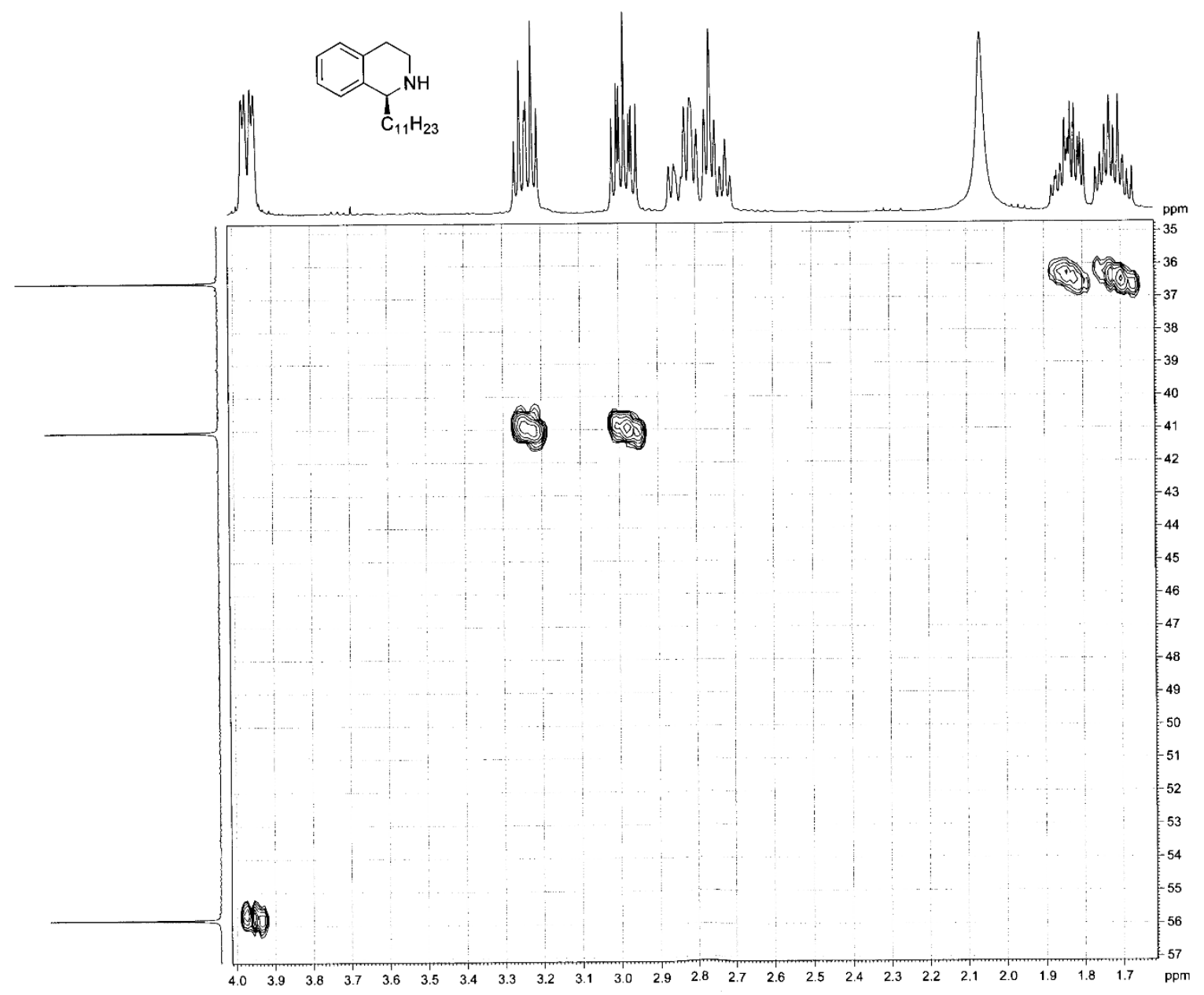

Figure S 95. HMQC spectrum ( $\left.\mathrm{CDCl}_{3}\right)$ of derivative (-)-9e. 
<smiles>COc1ccc(CC2NCCc3ccccc32)cc1</smiles>

(-)-9f

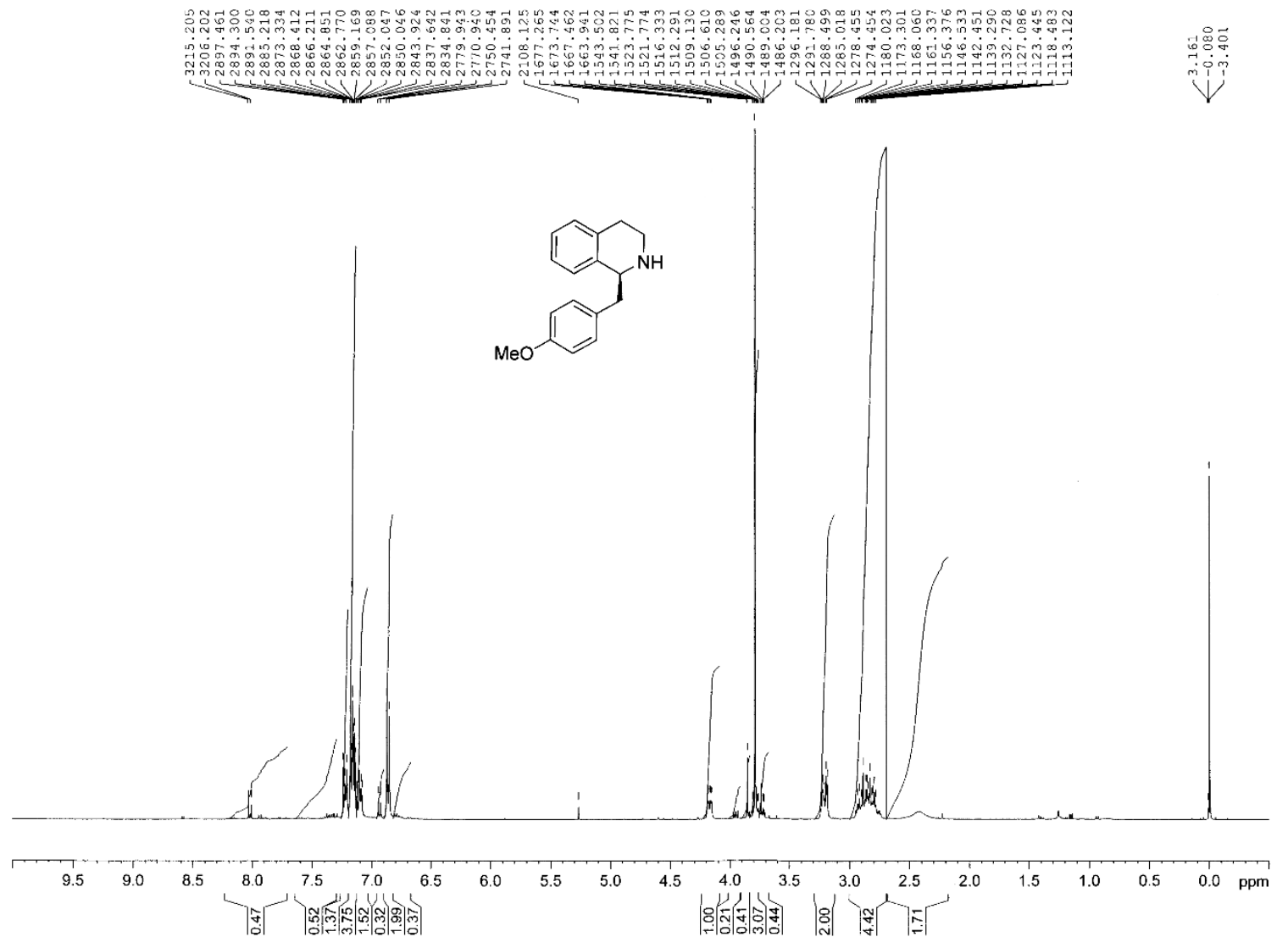

Figure S 96. ${ }^{1} \mathrm{H}$ NMR spectrum $\left(\mathrm{CDCl}_{3}, 400 \mathrm{MHz}\right)$ of derivative (-)-9f. 


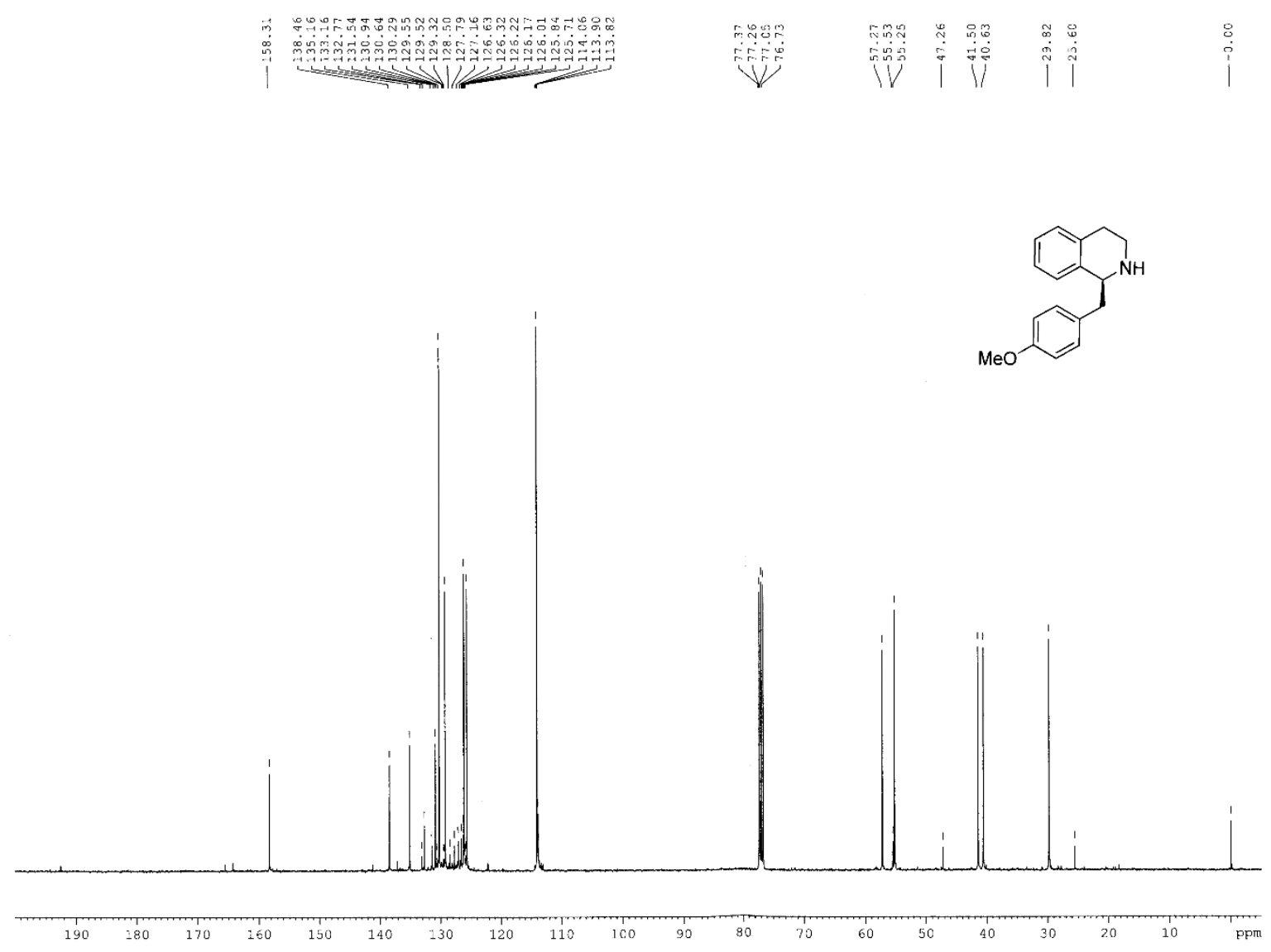

Figure $\mathrm{S} 97 .{ }^{13} \mathrm{C}$ NMR spectrum $\left(\mathrm{CDCl}_{3}, 100 \mathrm{MHz}\right)$ of derivative (-)-9f. 

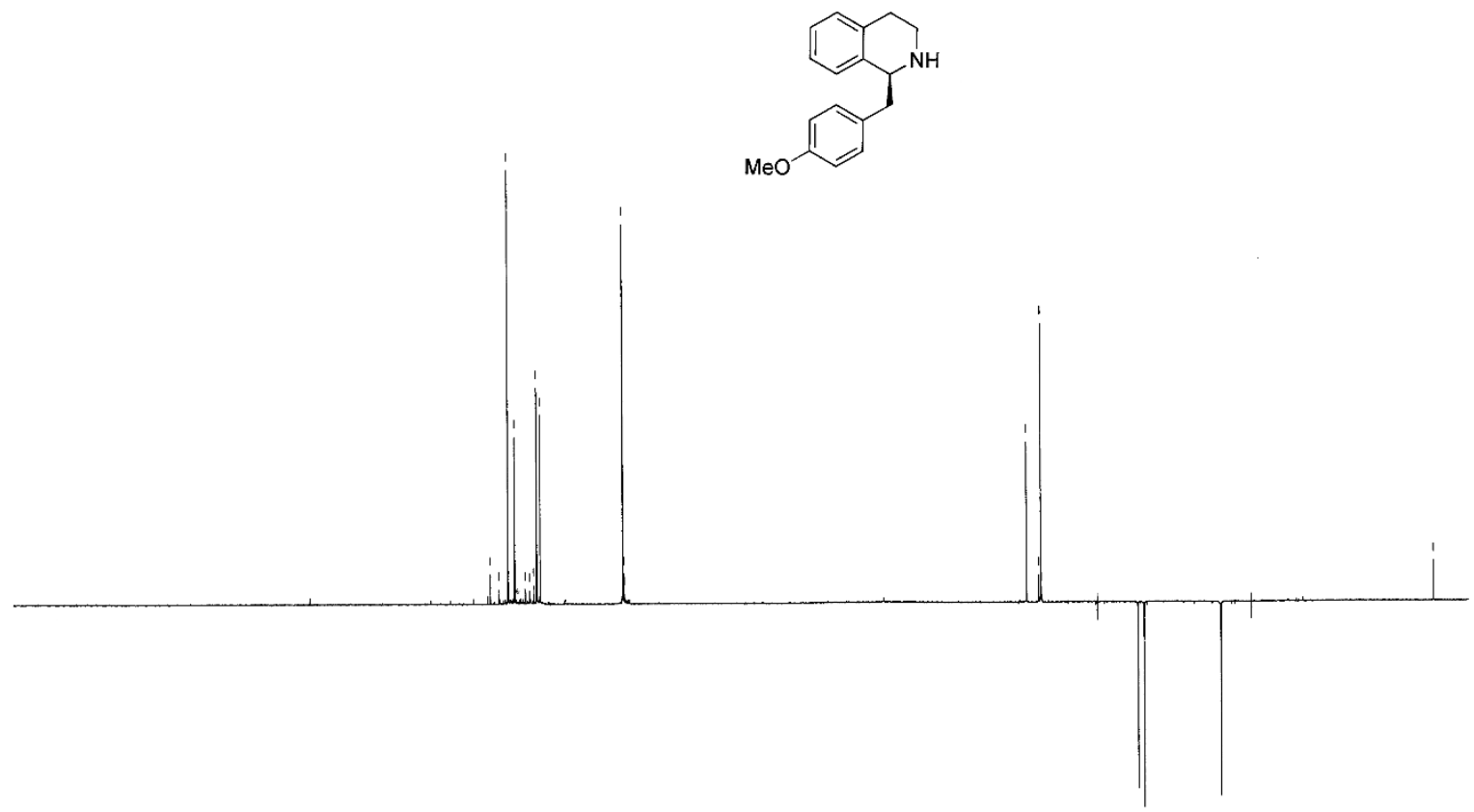

Figure S 98. DEPT C NMR spectrum ( $\left.\mathrm{CDCl}_{3}, 100 \mathrm{MHz}\right)$ of derivative (-)-9f. 
<smiles>COc1ccc(CC2NCCc3ccccc32)cc1OC</smiles>

(-)-9g

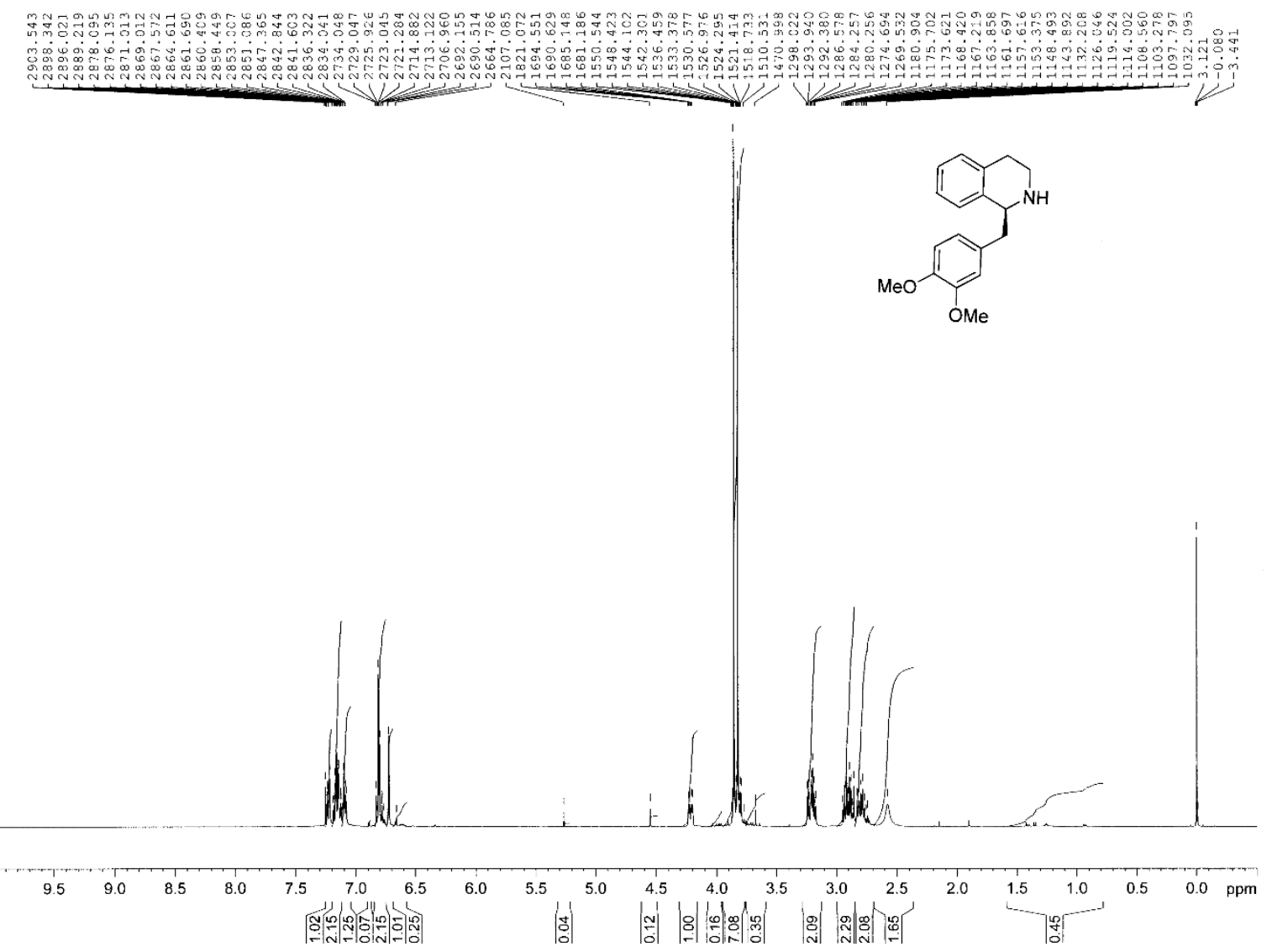

Figure S 99. ${ }^{1} \mathrm{H}$ NMR spectrum $\left(\mathrm{CDCl}_{3}, 400 \mathrm{MHz}\right)$ of derivative (-)-9g. 


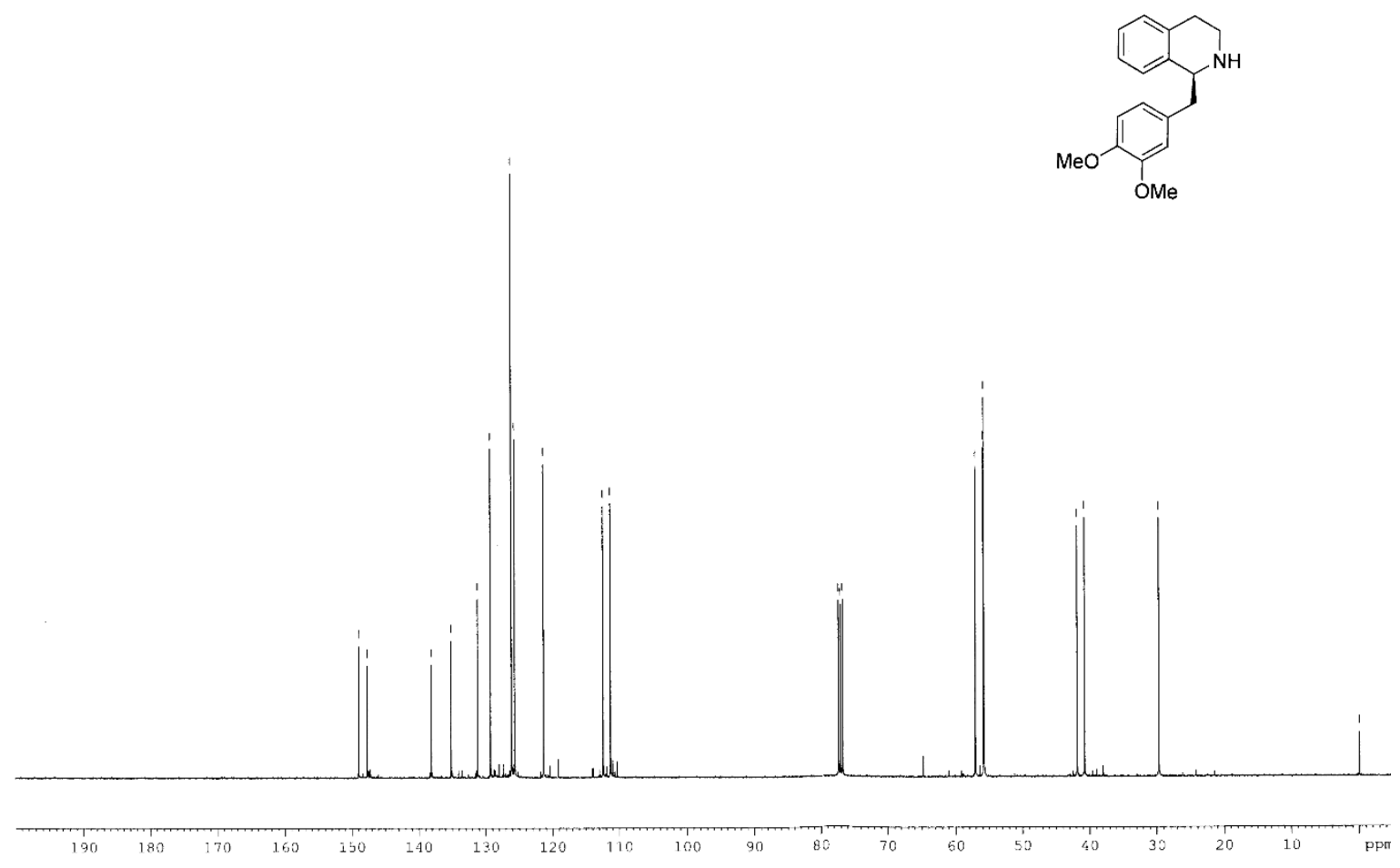

Figure $\mathbf{S} 100 .{ }^{13} \mathrm{C}$ NMR spectrum $\left(\mathrm{CDCl}_{3}, 100 \mathrm{MHz}\right)$ of derivative (-)-9g. 


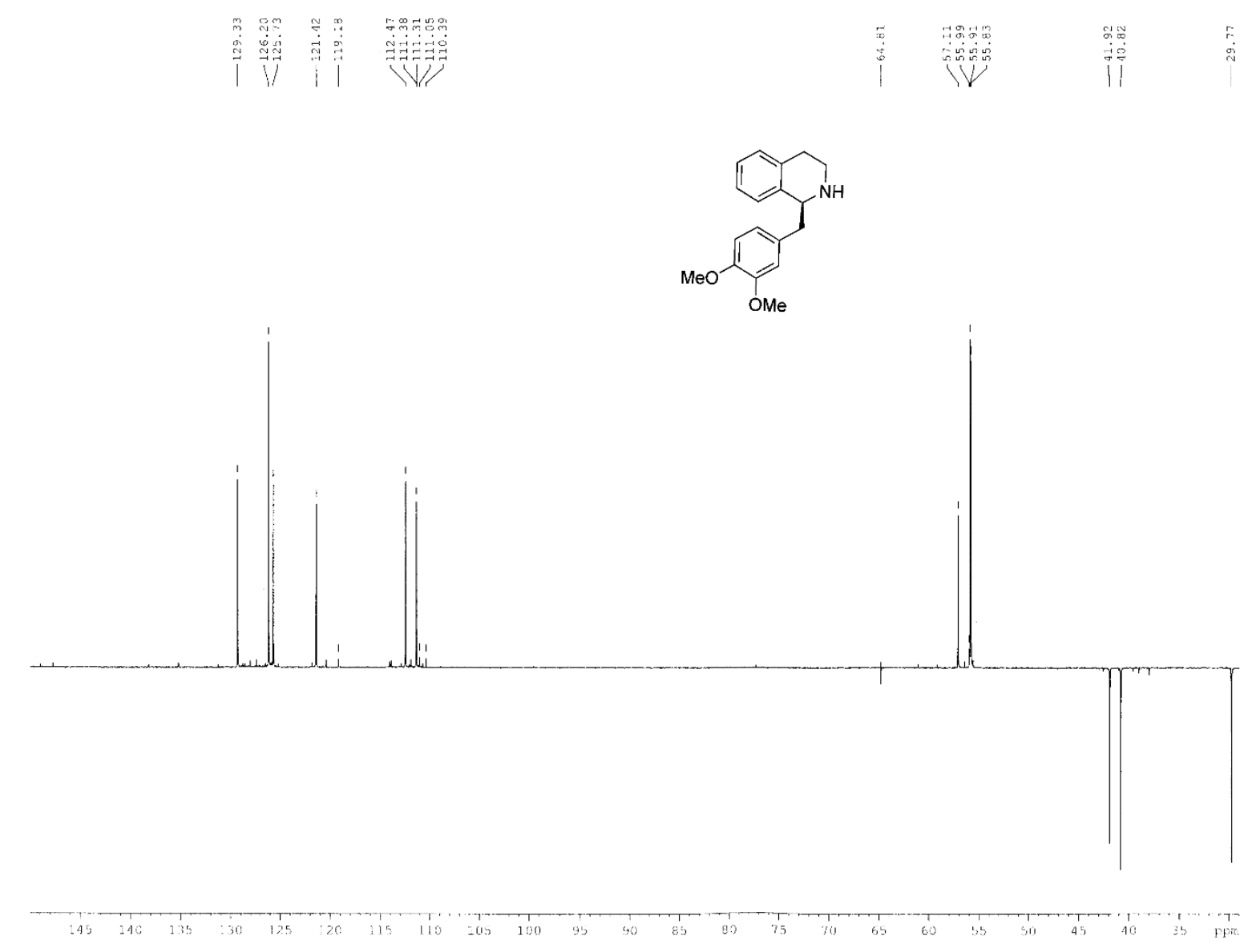

Figure $\mathbf{S}$ 101. DEPT -NMR spectrum ( $\left.\mathrm{CDCl}_{3}, 100 \mathrm{MHz}\right)$ of derivative (-)-9g. 


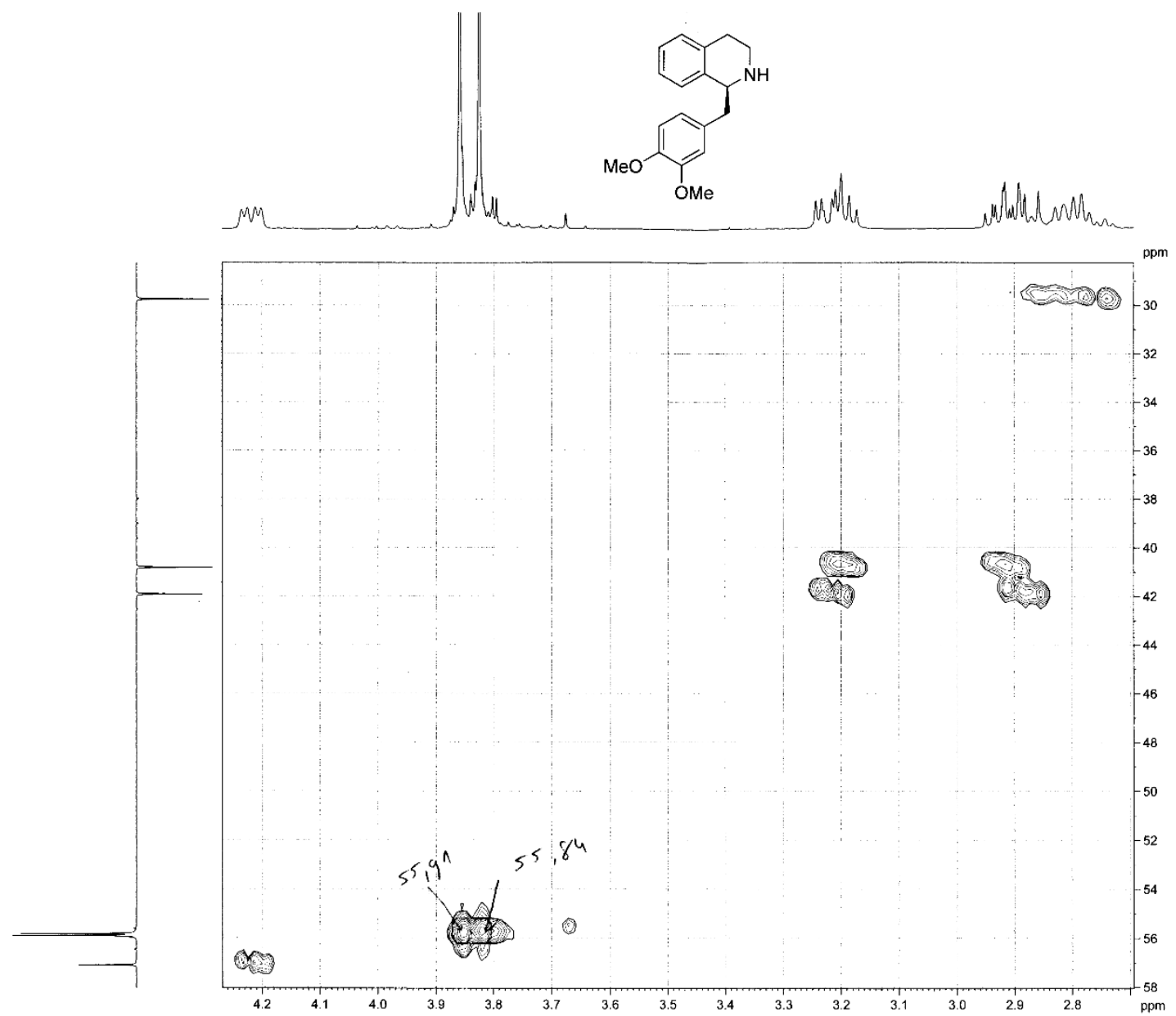

Figure S 102. $\mathrm{HMQC}$ spectrum $\left(\mathrm{CDCl}_{3}, 100 \mathrm{MHz}\right)$ of derivative (-)-9g. 


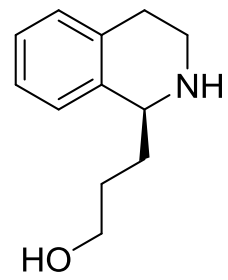

$(-)-9 h$

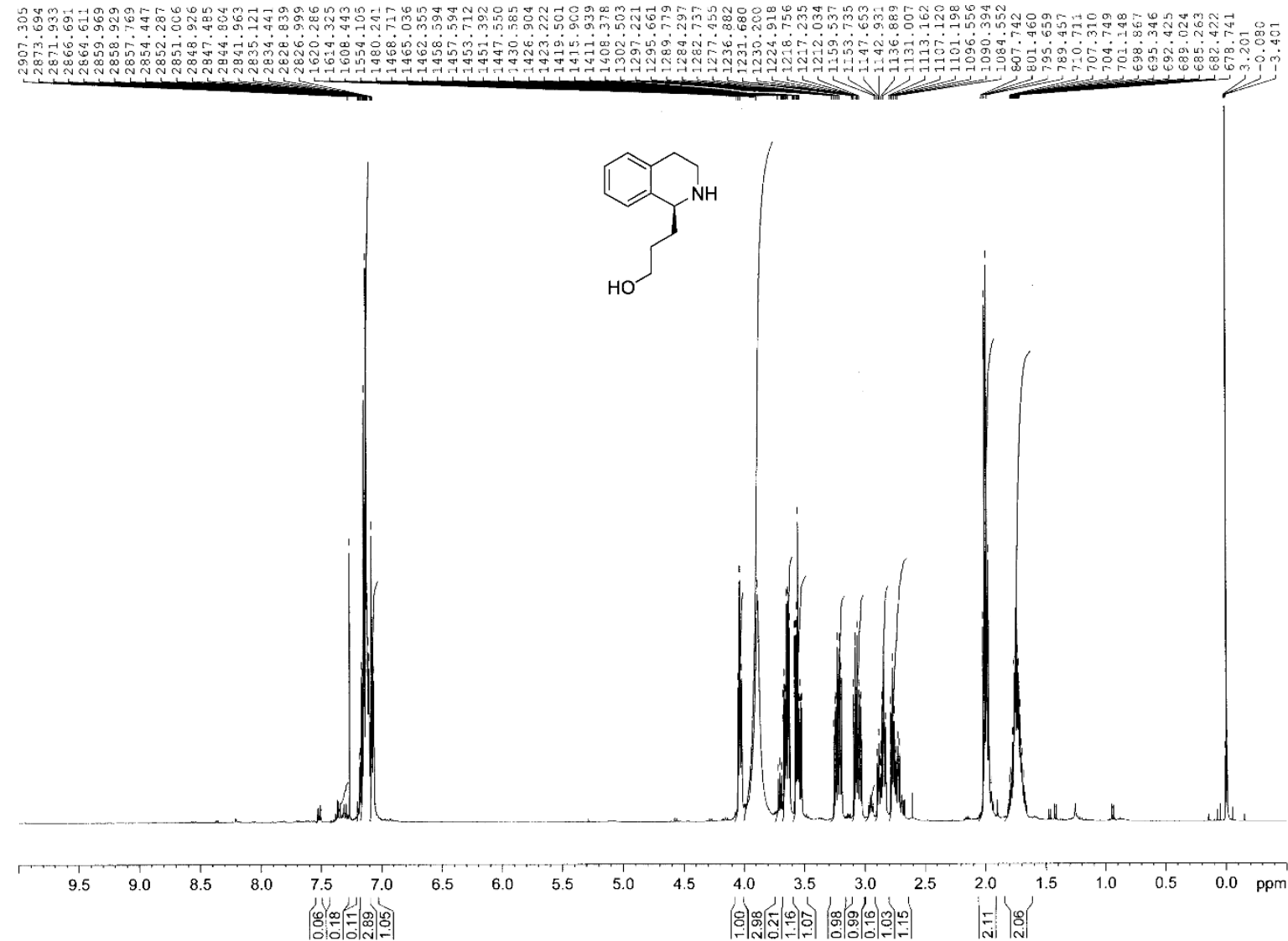

Figure S 103. ${ }^{1} \mathrm{H}$ NMR spectrum $\left(\mathrm{CDCl}_{3}, 400 \mathrm{MHz}\right)$ of derivative (-)-9h. 


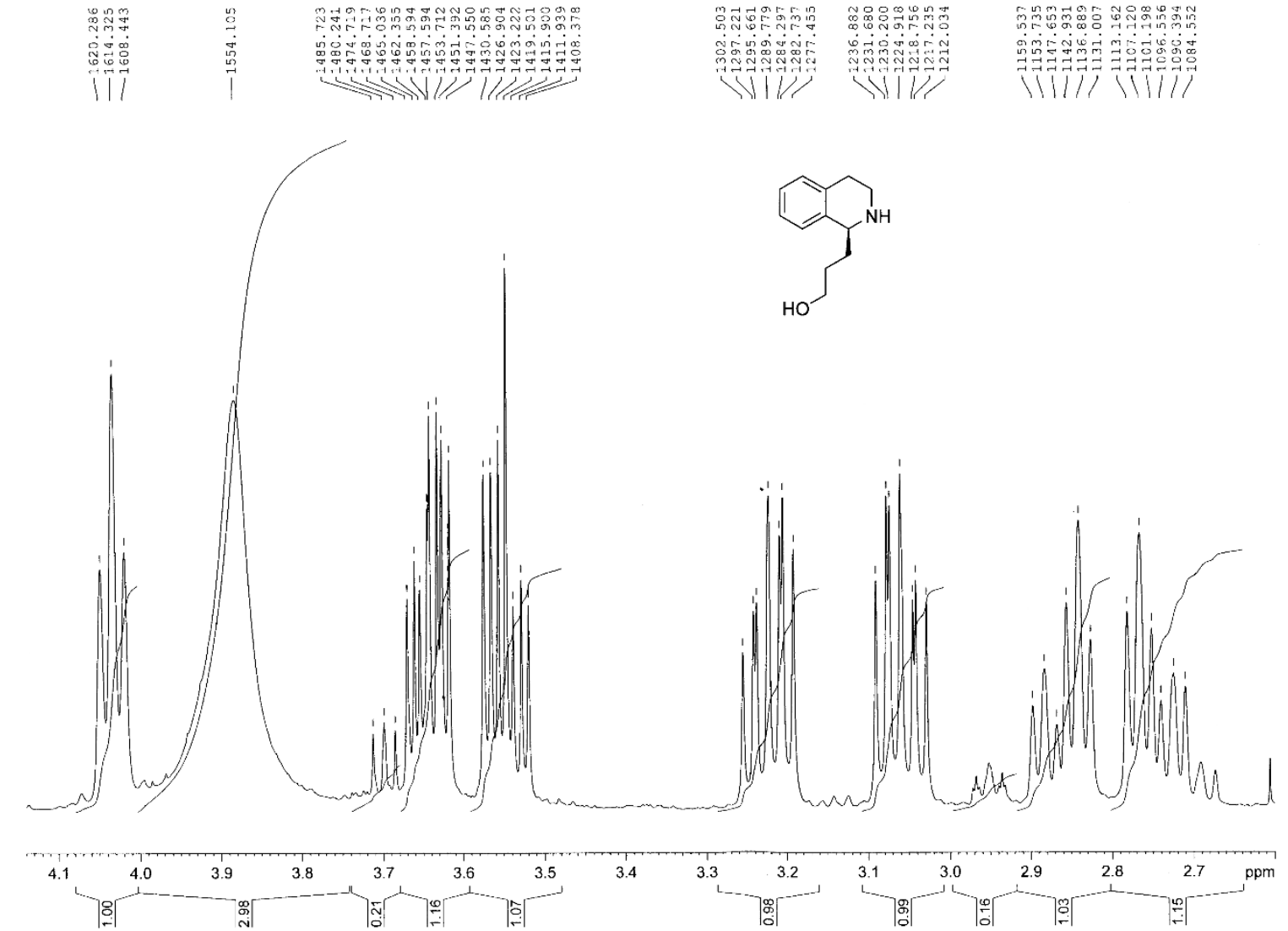

Figure S 104. ${ }^{1} \mathrm{H}$ NMR spectrum $\left(\mathrm{CDCl}_{3}, 400 \mathrm{MHz}\right)$ of derivative (-)-9h. 


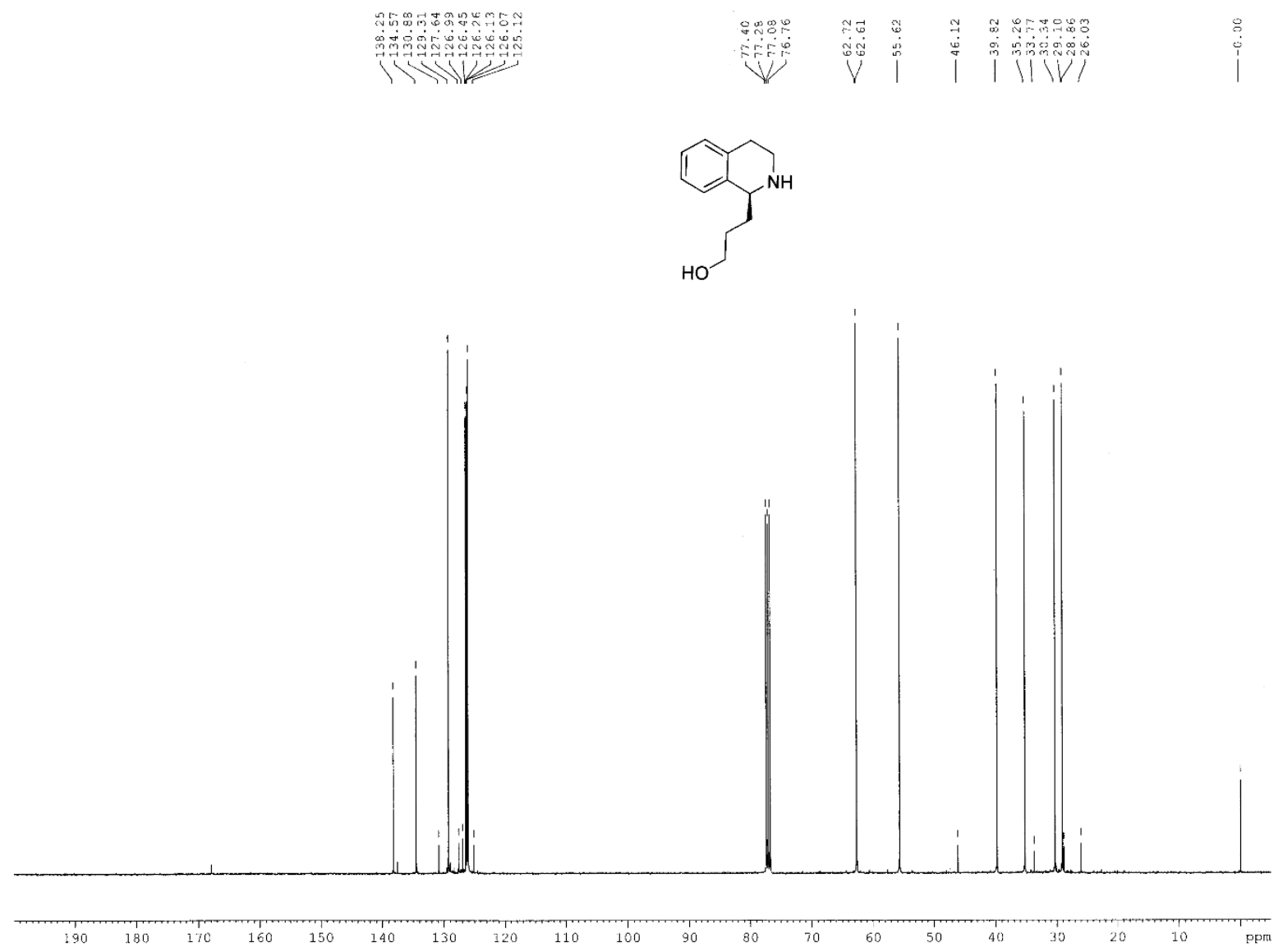

Figure S $105 .{ }^{13} \mathrm{C}$ NMR spectrum $\left(\mathrm{CDCl}_{3}, 100 \mathrm{MHz}\right)$ of derivative (-)-9h. 


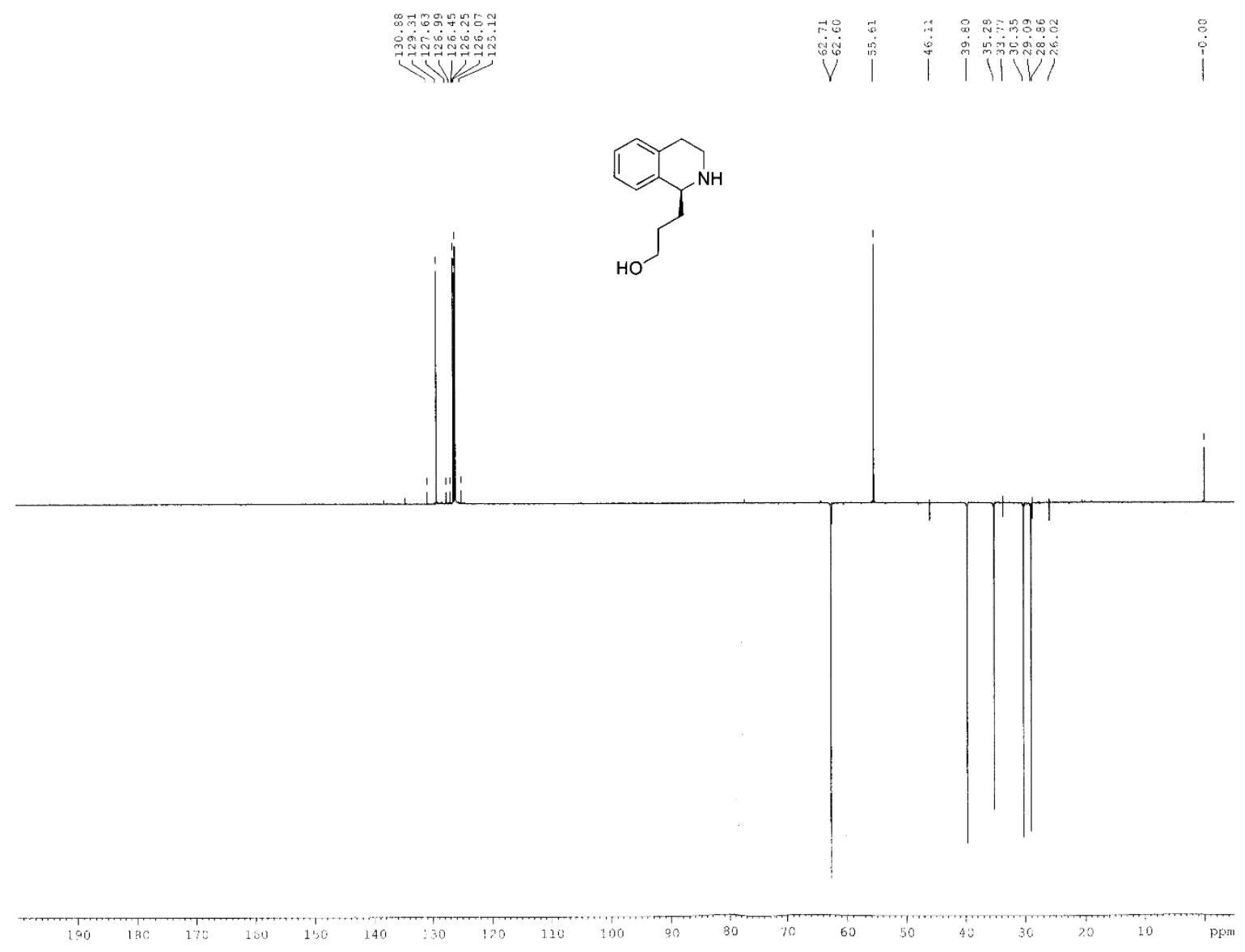

Figure S 106. DEPT C NMR spectrum $\left(\mathrm{CDCl}_{3}, 100 \mathrm{MHz}\right)$ of derivative (-)-9h. 


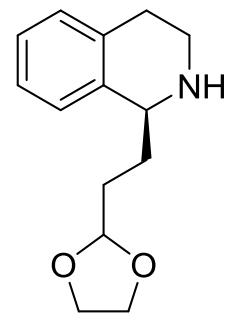

$(-)-9 \mathbf{i}$

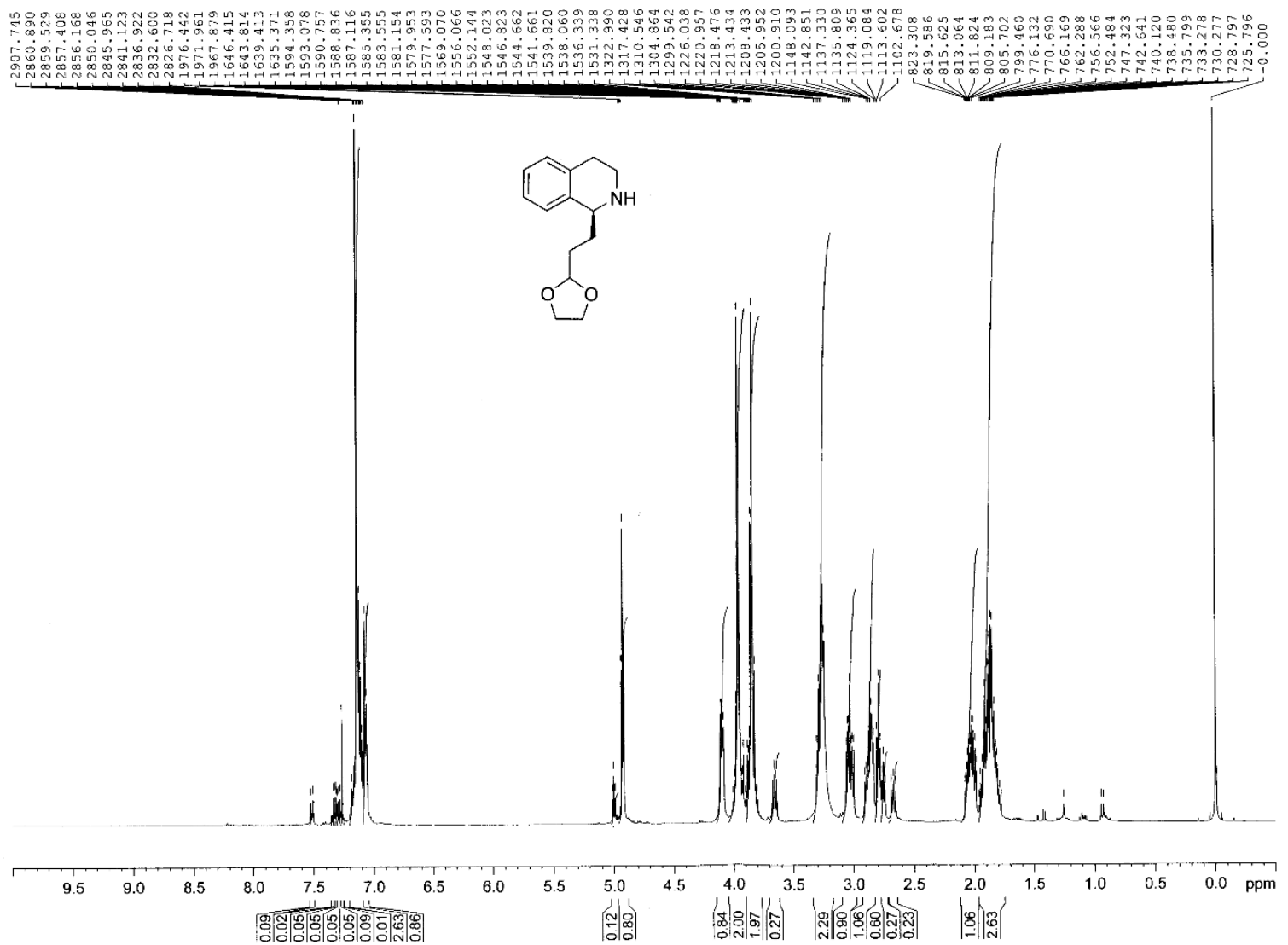

Figure S 107. ${ }^{1} \mathrm{H}$ NMR spectrum $\left(\mathrm{CDCl}_{3}, 400 \mathrm{MHz}\right)$ of derivative (-)-9i. 


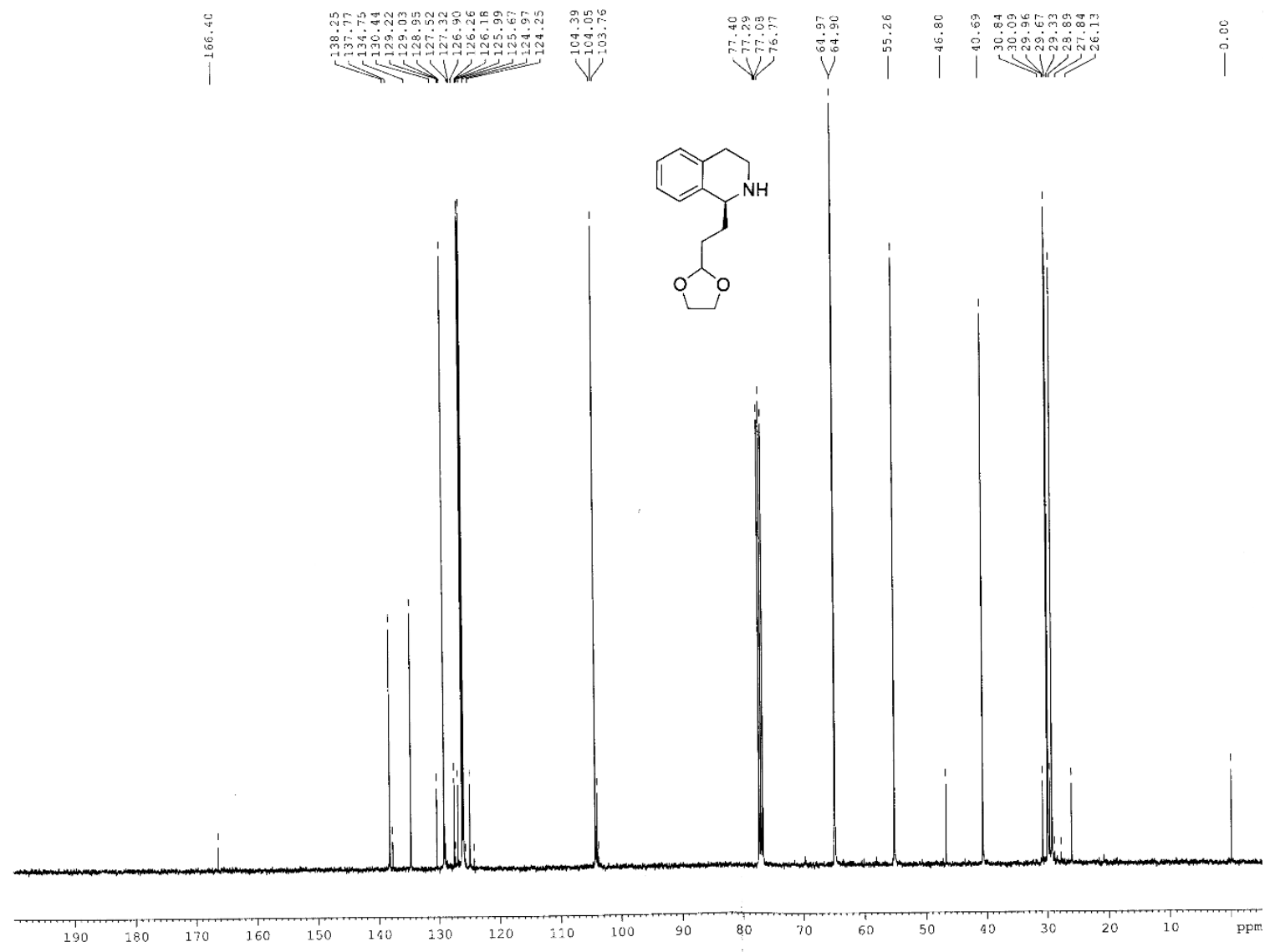

Figure S 108. ${ }^{13} \mathrm{C}$ NMR spectrum $\left(\mathrm{CDCl}_{3}, 100 \mathrm{MHz}\right)$ of derivative (-)-9i. 
S111

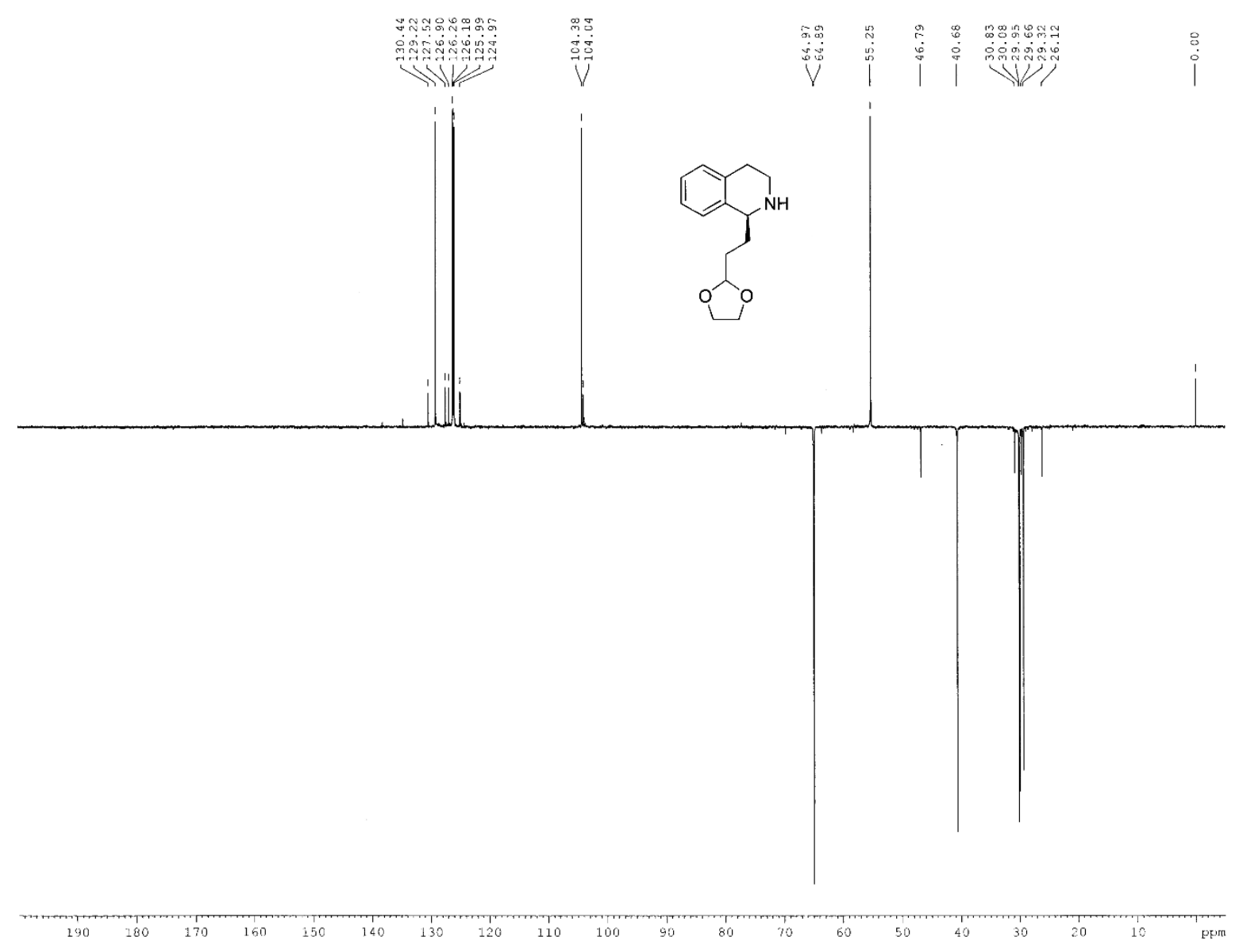

Figure S 109. DEPT C NMR spectrum ( $\left.\mathrm{CDCl}_{3}, 100 \mathrm{MHz}\right)$ of derivative (-)-gi. 


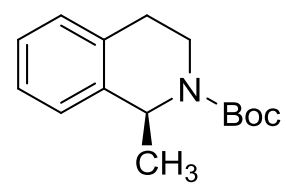

(+)-10a

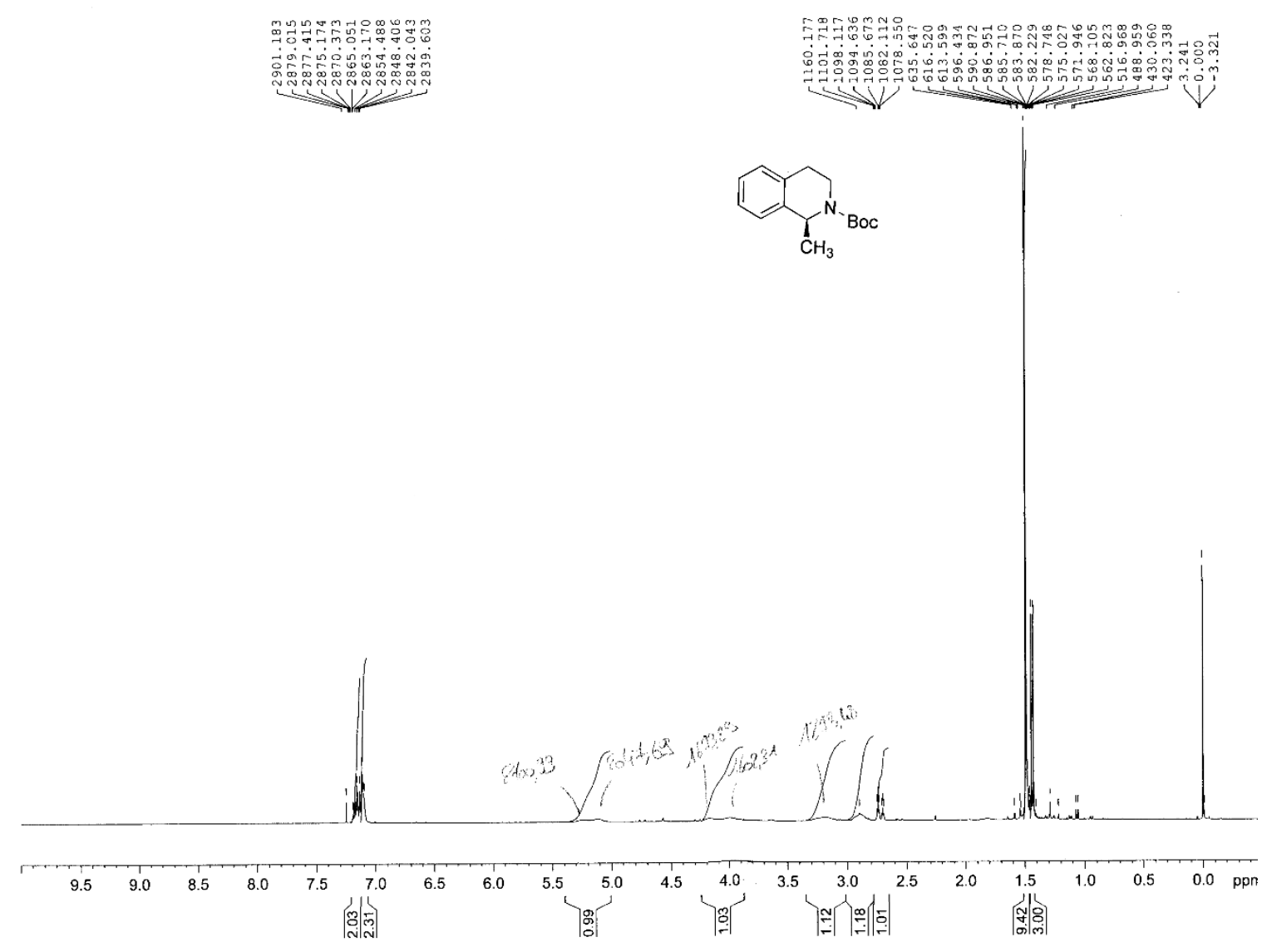

Figure S 110. ${ }^{1} \mathrm{H}$ NMR spectrum $\left(\mathrm{CDCl}_{3}, 400 \mathrm{MHz}\right)$ of derivative (+)-10a. 


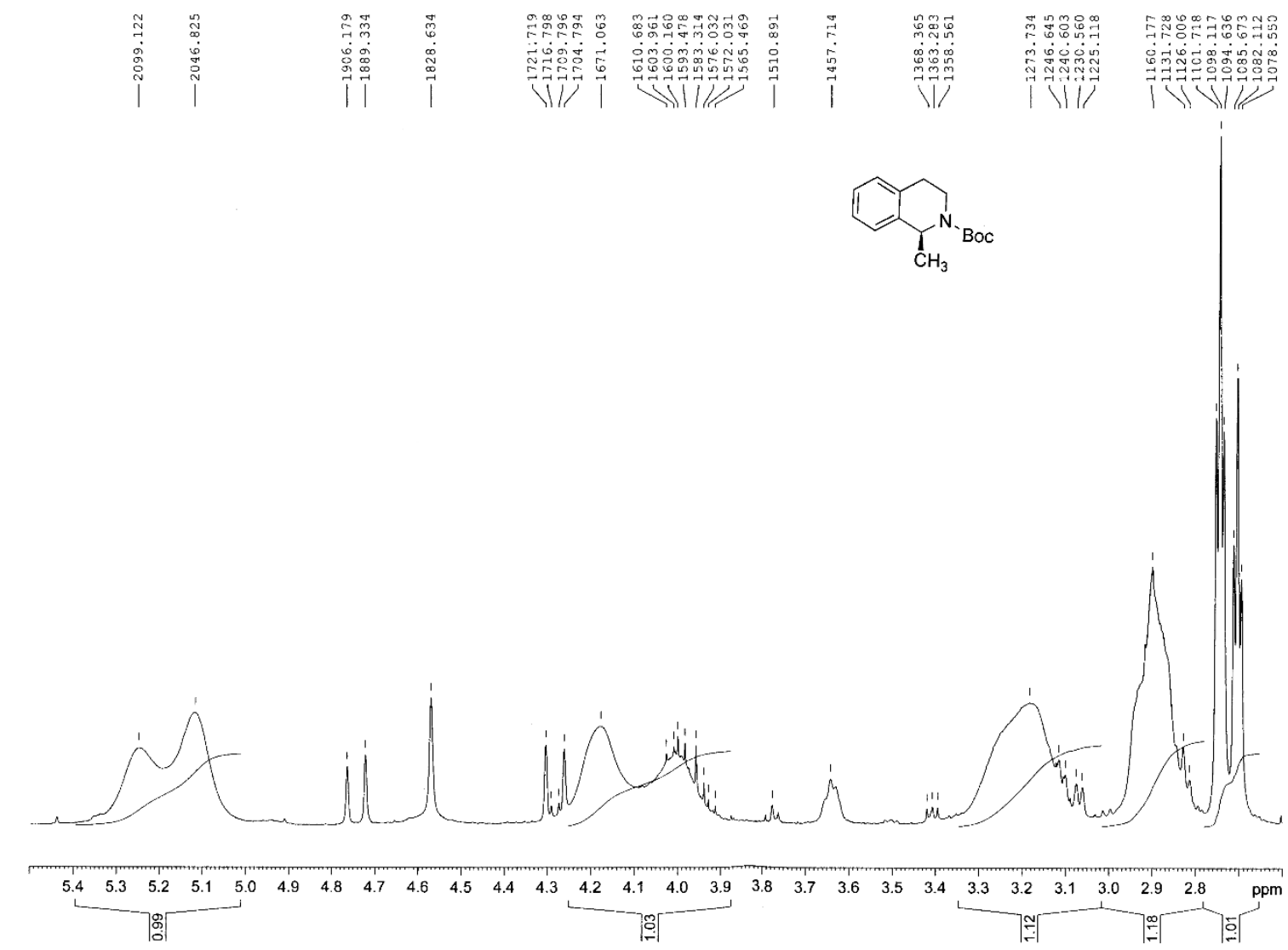

Figure S 111. ${ }^{1} \mathrm{H}$ NMR spectrum $\left(\mathrm{CDCl}_{3}, 400 \mathrm{MHz}\right)$ of derivative (+)-10a. 
S114

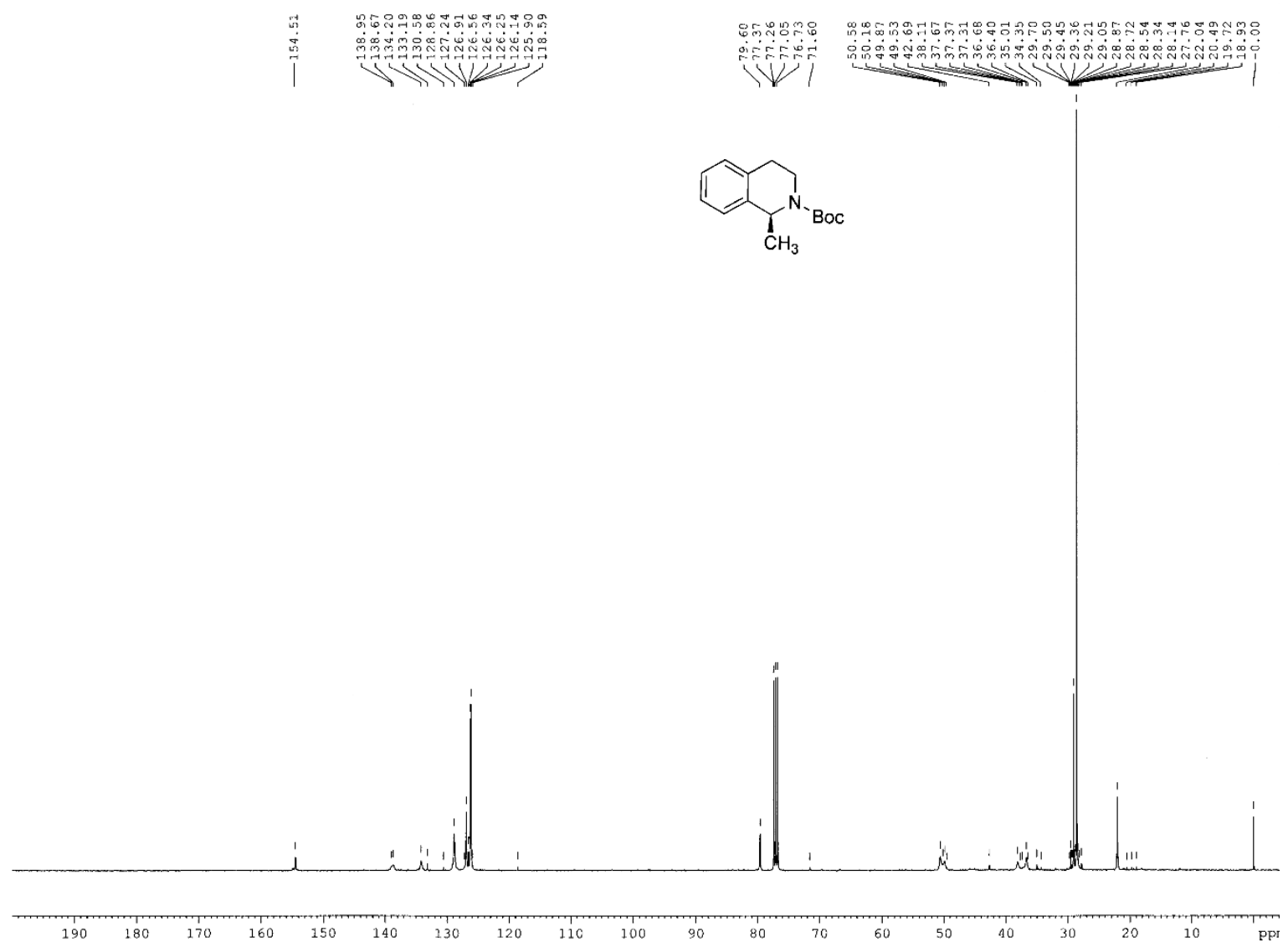

Figure S $112 .{ }^{13} \mathrm{C}$ NMR spectrum $\left(\mathrm{CDCl}_{3}, 100 \mathrm{MHz}\right)$ of derivative $(+)-10 a$. 


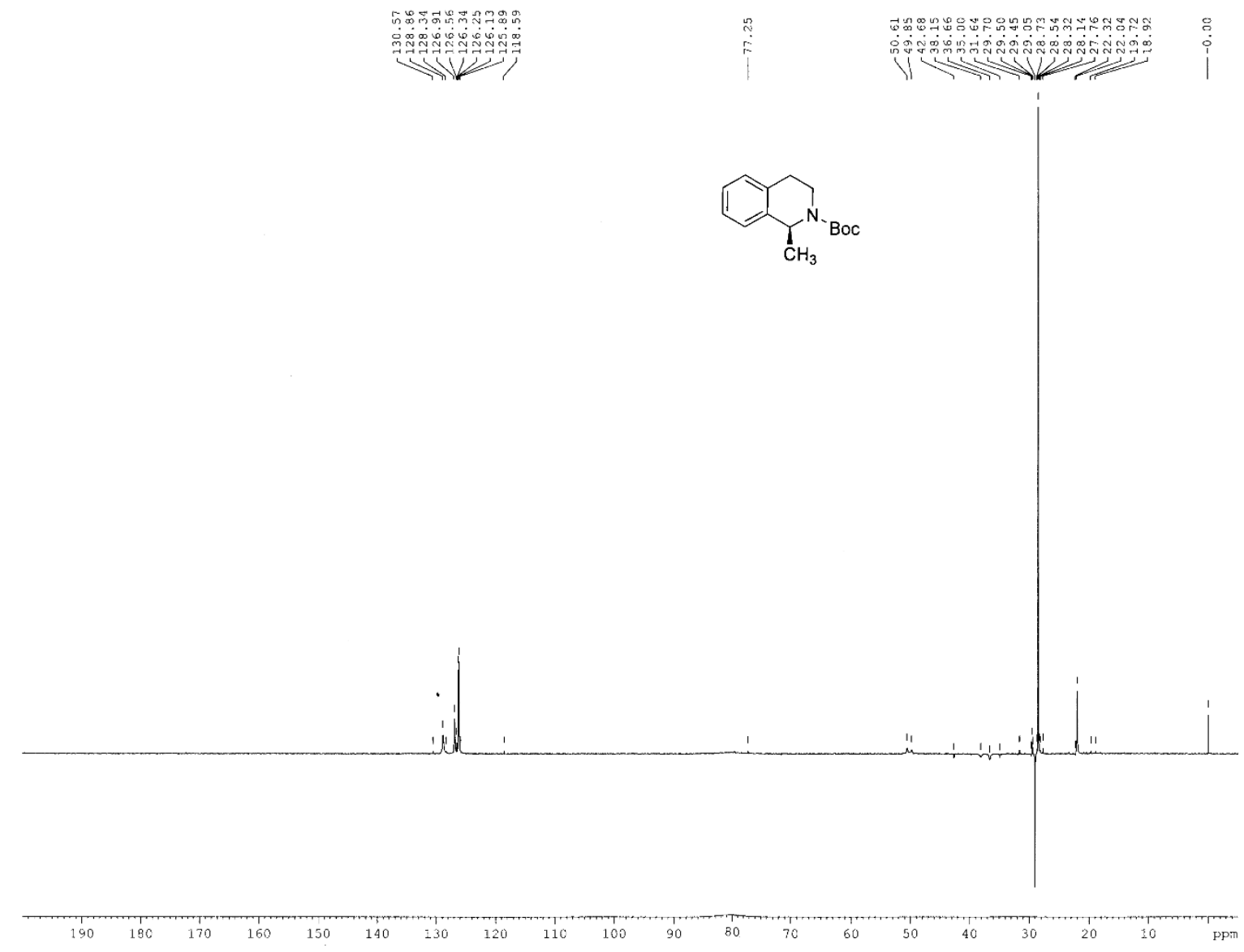

Figure S 113. DEPT C NMR spectrum $\left(\mathrm{CDCl}_{3}, 100 \mathrm{MHz}\right)$ of derivative $(+)-10 a$. 


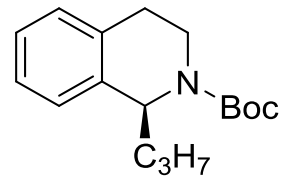

$(+)-10 b$

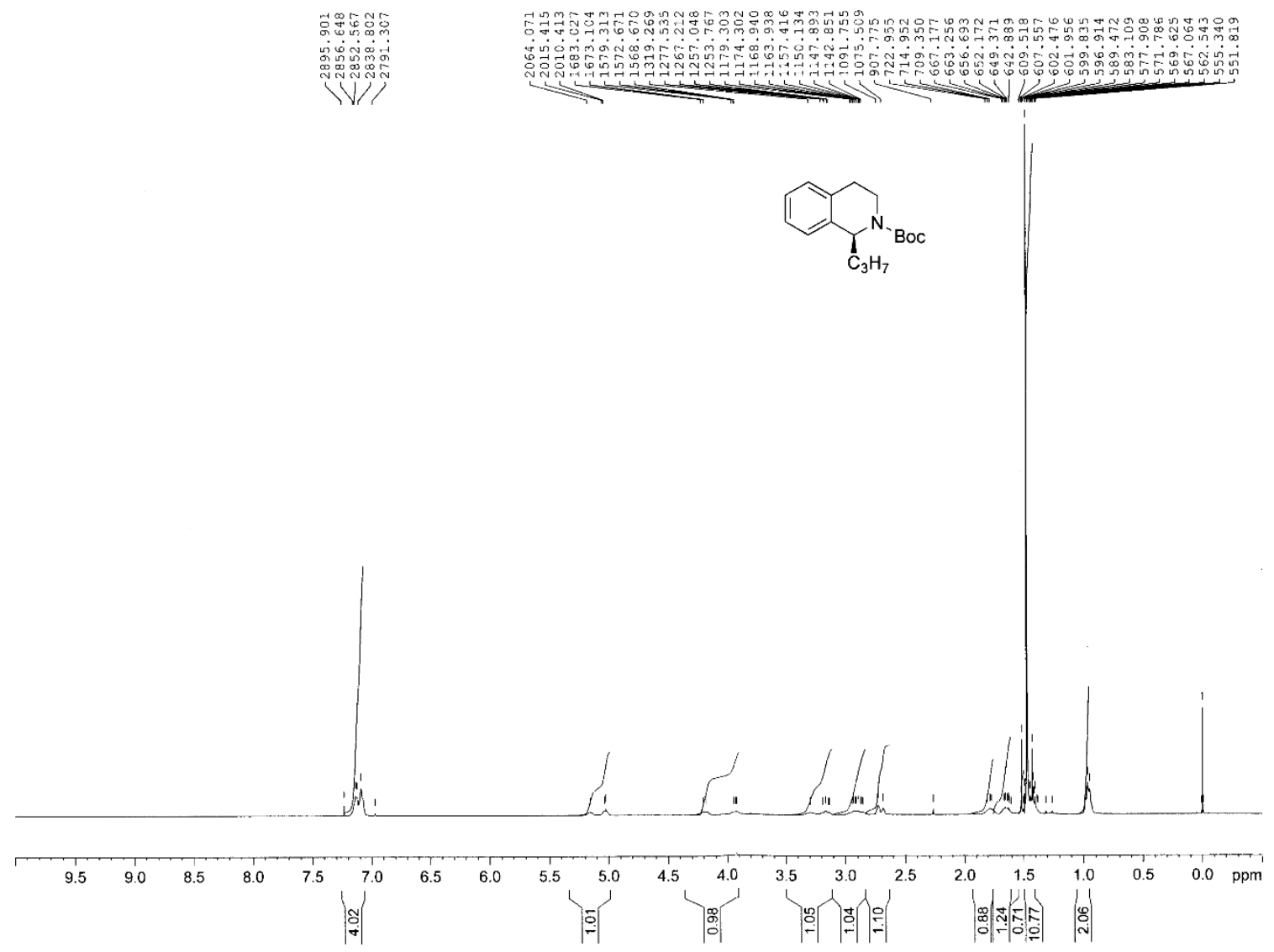

Figure S 114. ${ }^{1} \mathrm{H}$ NMR spectrum $\left(\mathrm{CDCl}_{3}, 400 \mathrm{MHz}\right)$ of derivative (+)-10b. 


$$
\text { (1) }
$$

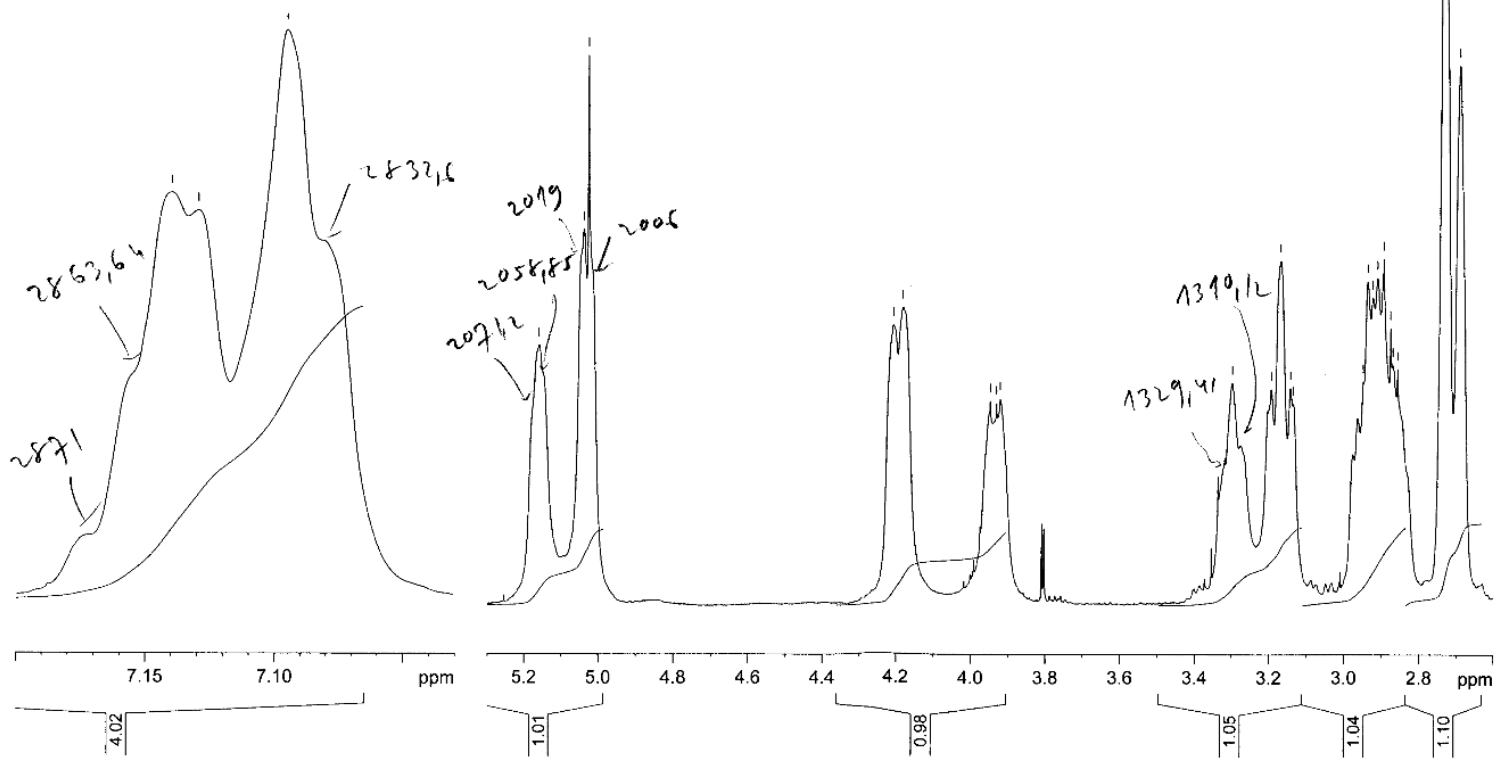

Figure S 115. ${ }^{1} \mathrm{H}$ NMR spectrum $\left(\mathrm{CDCl}_{3}, 400 \mathrm{MHz}\right)$ of derivative (+)-10b. 


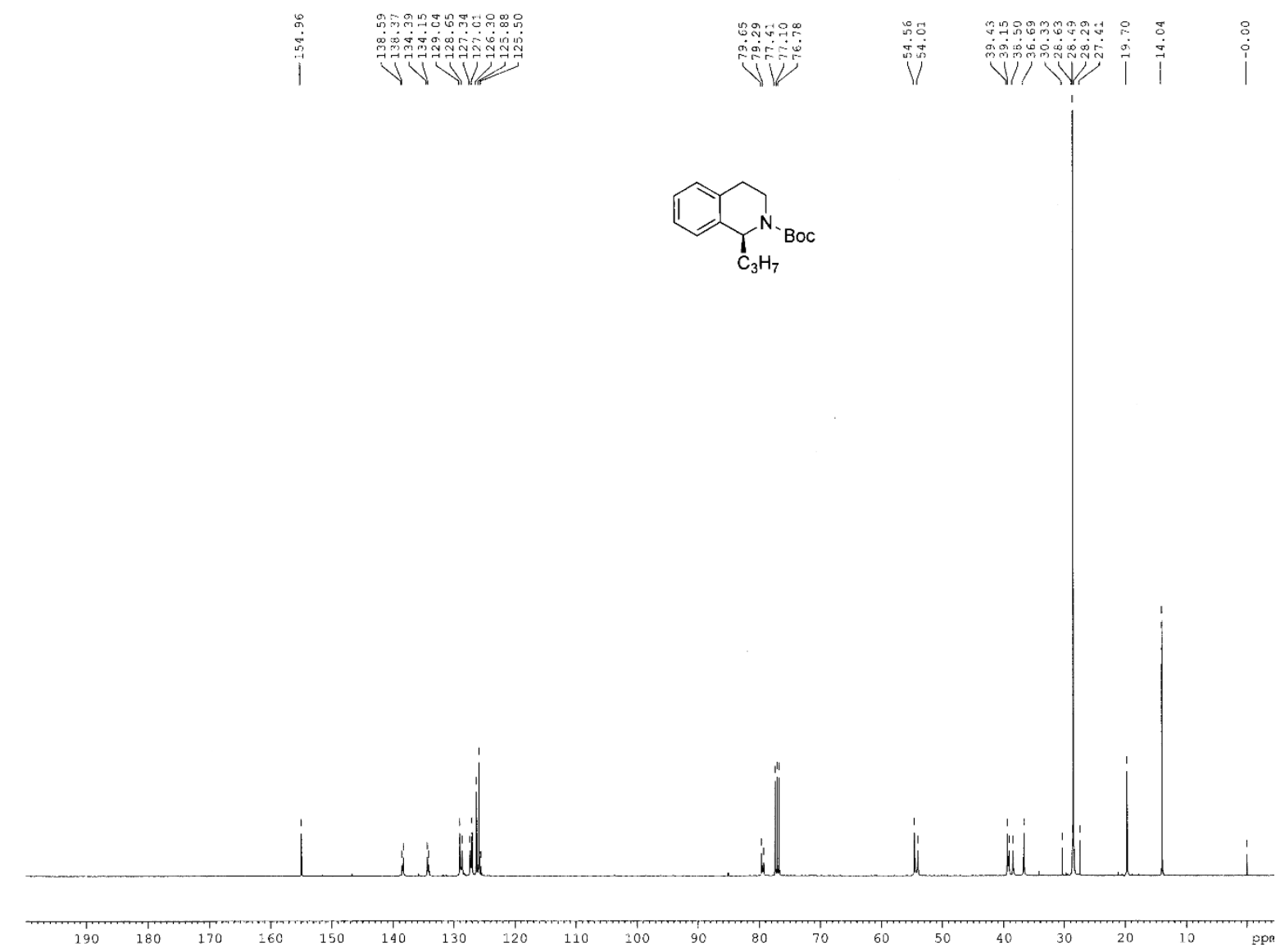

Figure S 116. ${ }^{13} \mathrm{C} \mathrm{NMR}$ spectrum $\left(\mathrm{CDCl}_{3}, 100 \mathrm{MHz}\right)$ of derivative $(+)-\mathbf{1 0 b}$. 


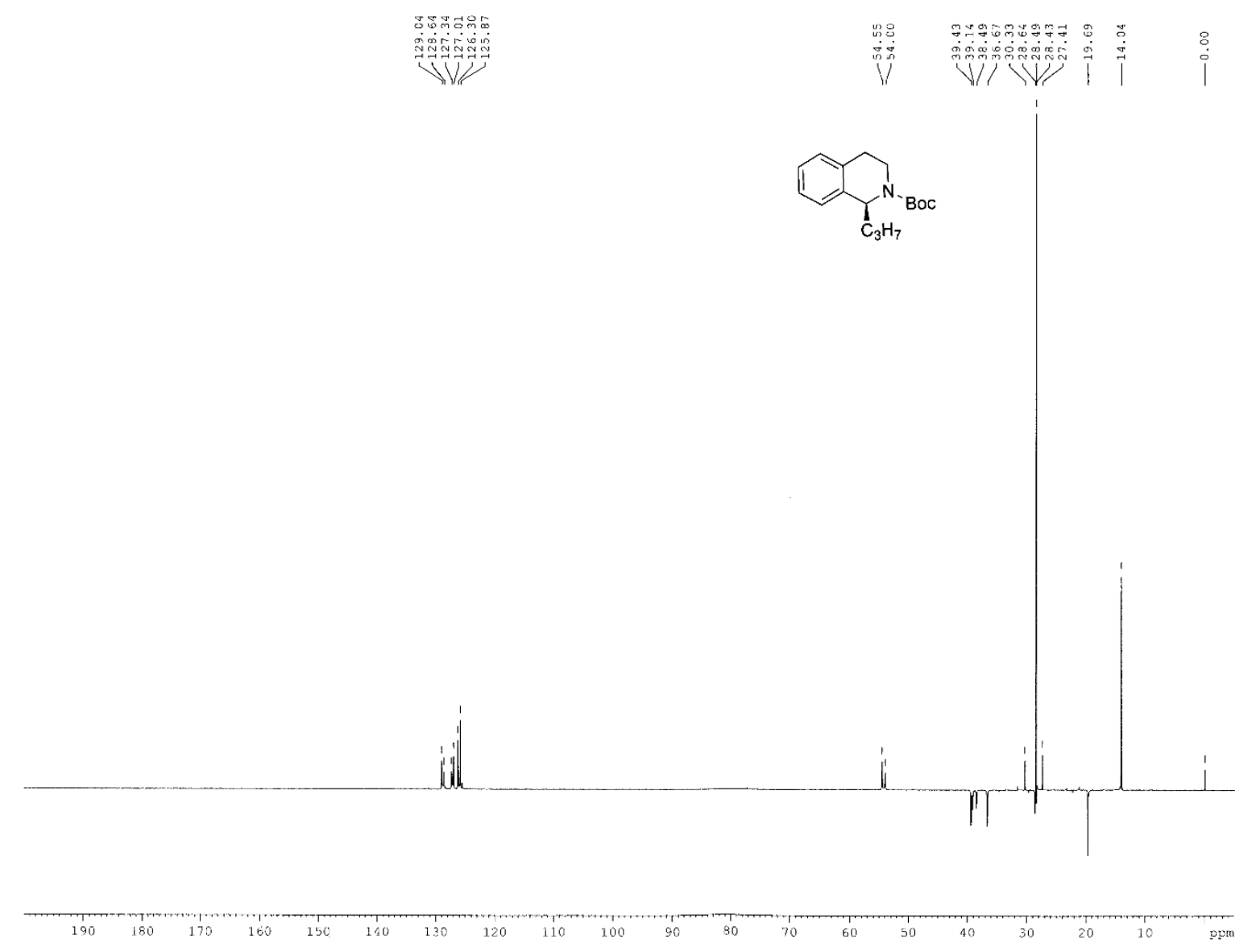

Figure S 117. DEPT C-NMR spectrum ( $\left.\mathrm{CDCl}_{3}, 100 \mathrm{MHz}\right)$ of derivative $(+)-\mathbf{1 0 b}$. 
<smiles>CC(C)(C)OC(=O)N1CCc2ccccc2[C@H]1[15CH3]</smiles>

$(+)-10 c$

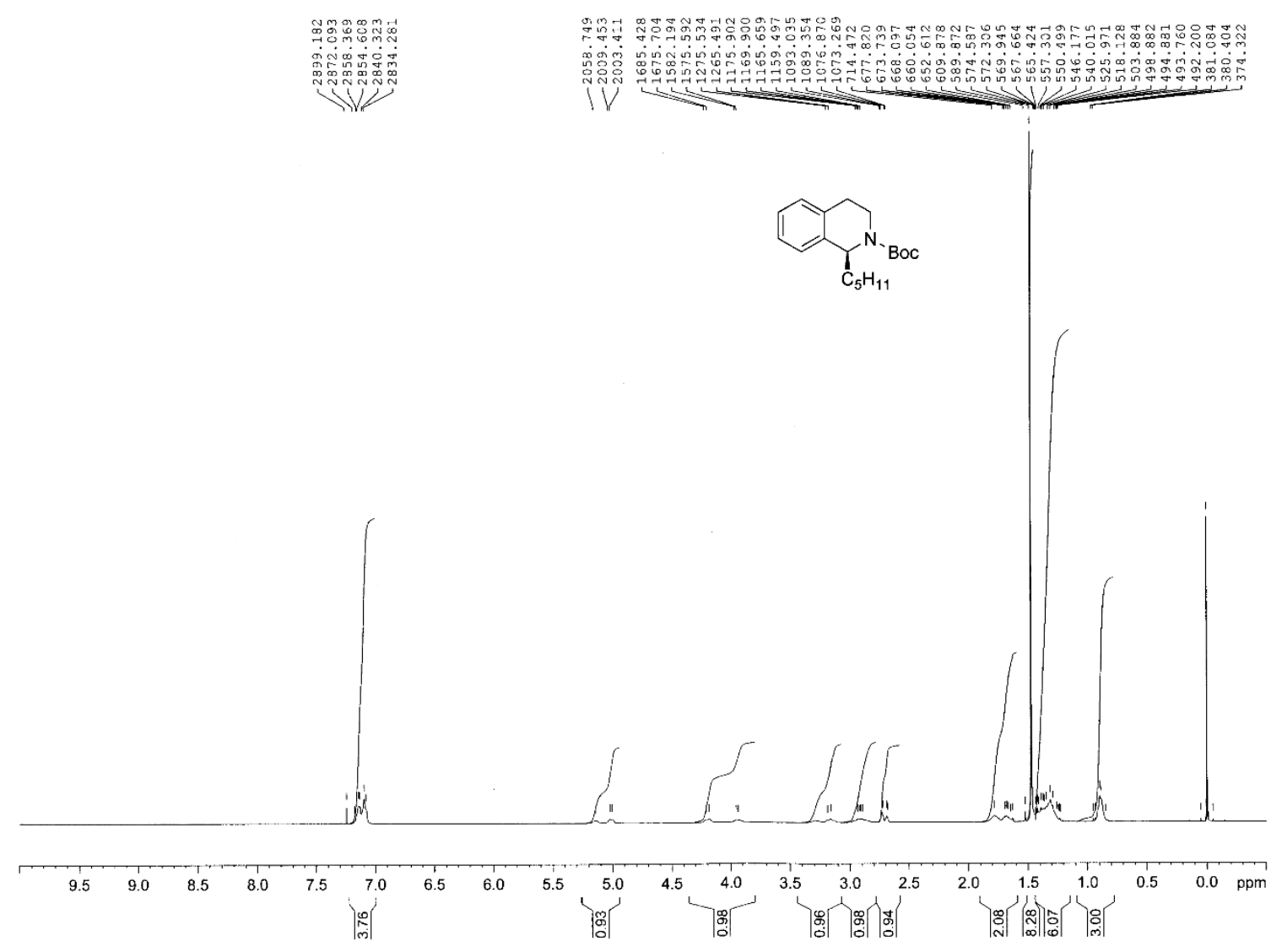

Figure S 118. ${ }^{1} \mathrm{H}$ NMR spectrum $\left(\mathrm{CDCl}_{3}, 400 \mathrm{MHz}\right)$ of derivative $(+)-10 \mathrm{c}$. 
S121

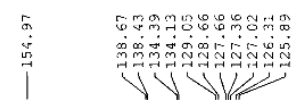

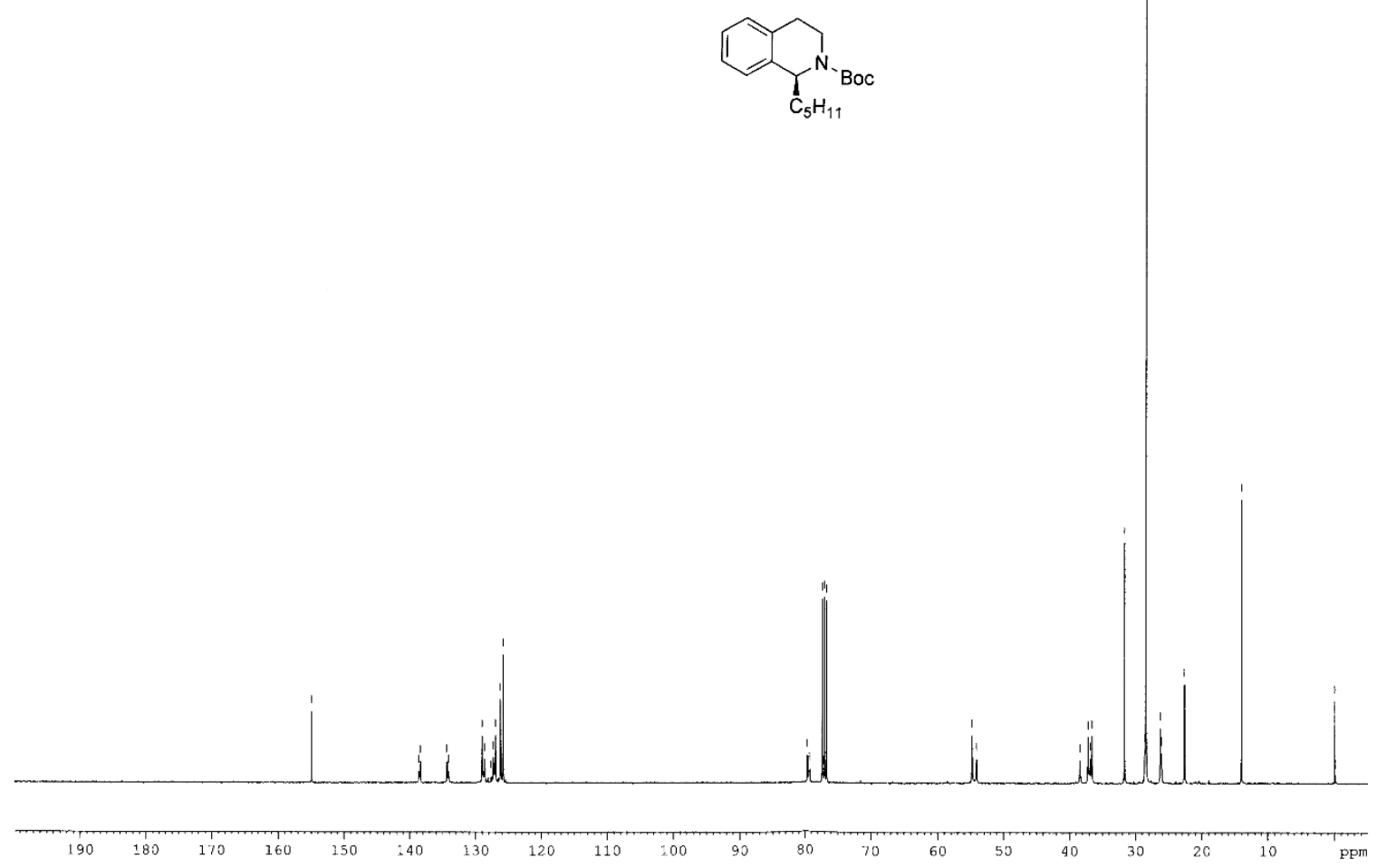

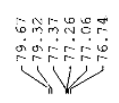

V|

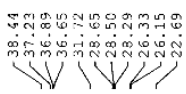

i

Figure S 119. ${ }^{13} \mathrm{C}$ NMR spectrum $\left(\mathrm{CDCl}_{3}, 100 \mathrm{MHz}\right)$ of derivative $(+)-10 \mathrm{c}$. 
S122

草号

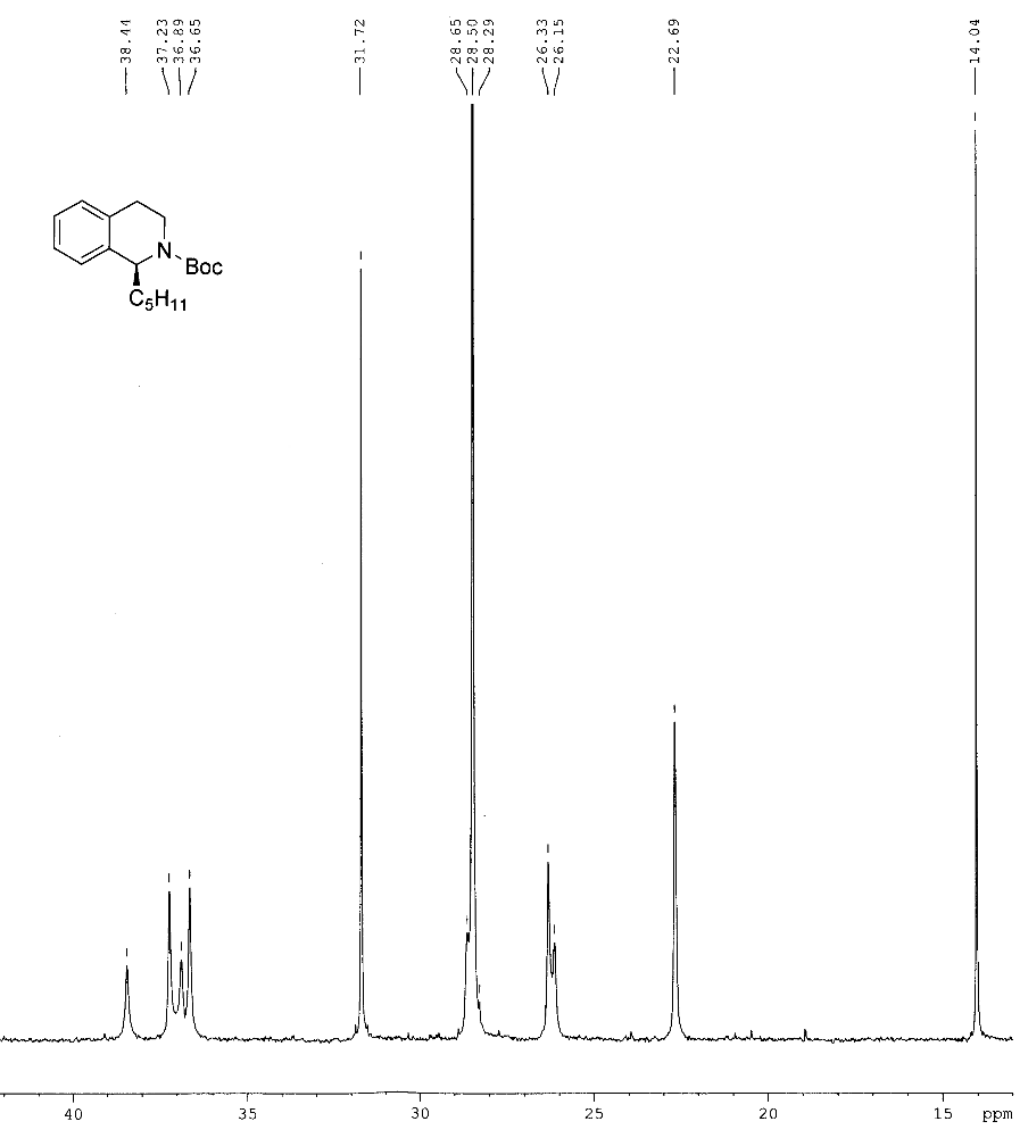

Figure $\mathrm{S}$ 120. ${ }^{13} \mathrm{C}$ NMR spectrum $\left(\mathrm{CDCl}_{3}, 100 \mathrm{MHz}\right)$ of derivative (+)-10c. 


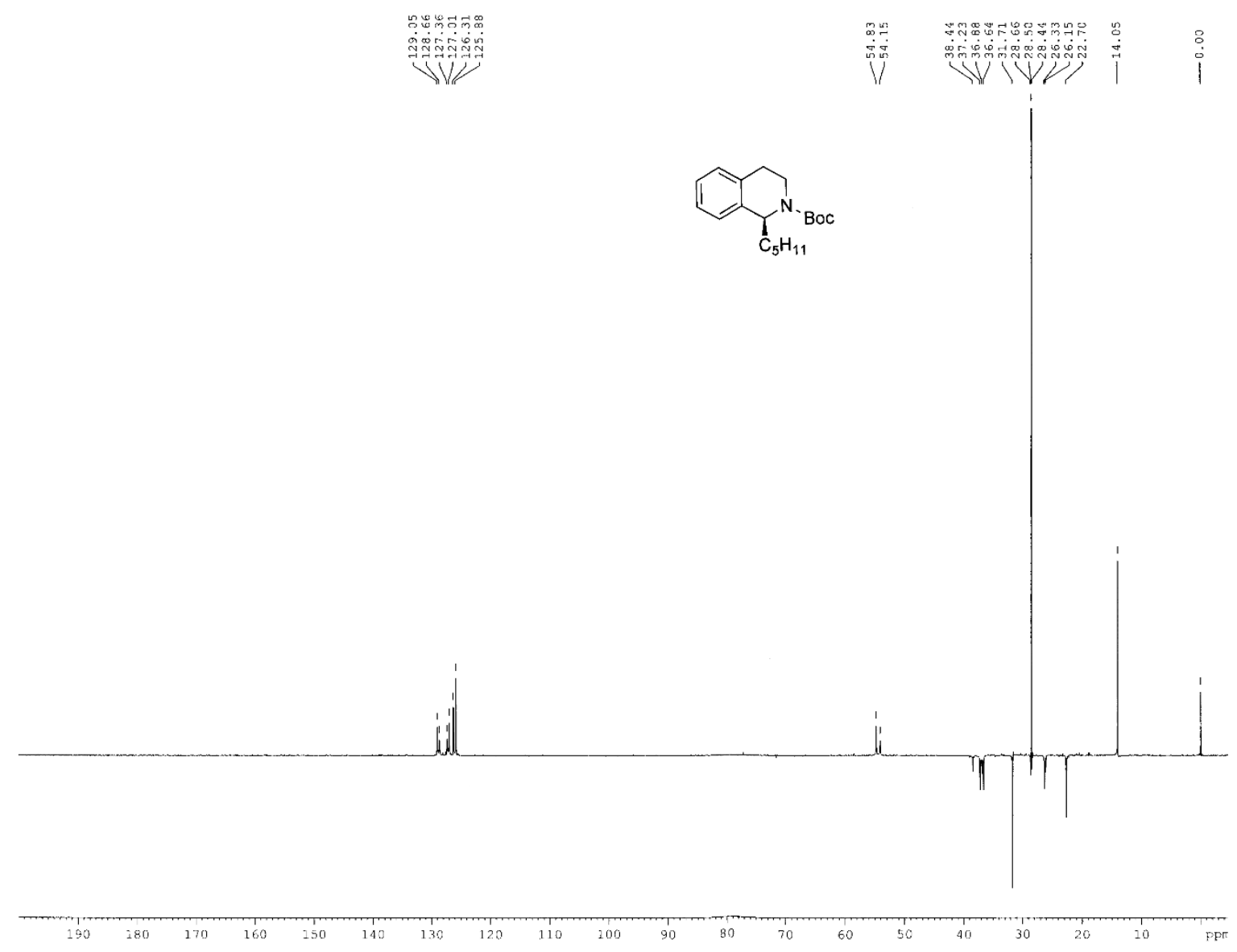

Figure $\mathrm{S}$ 121. DEPT C-NMR spectrum $\left(\mathrm{CDCl}_{3}, 100 \mathrm{MHz}\right)$ of derivative (+)-10c. 


\section{S124}

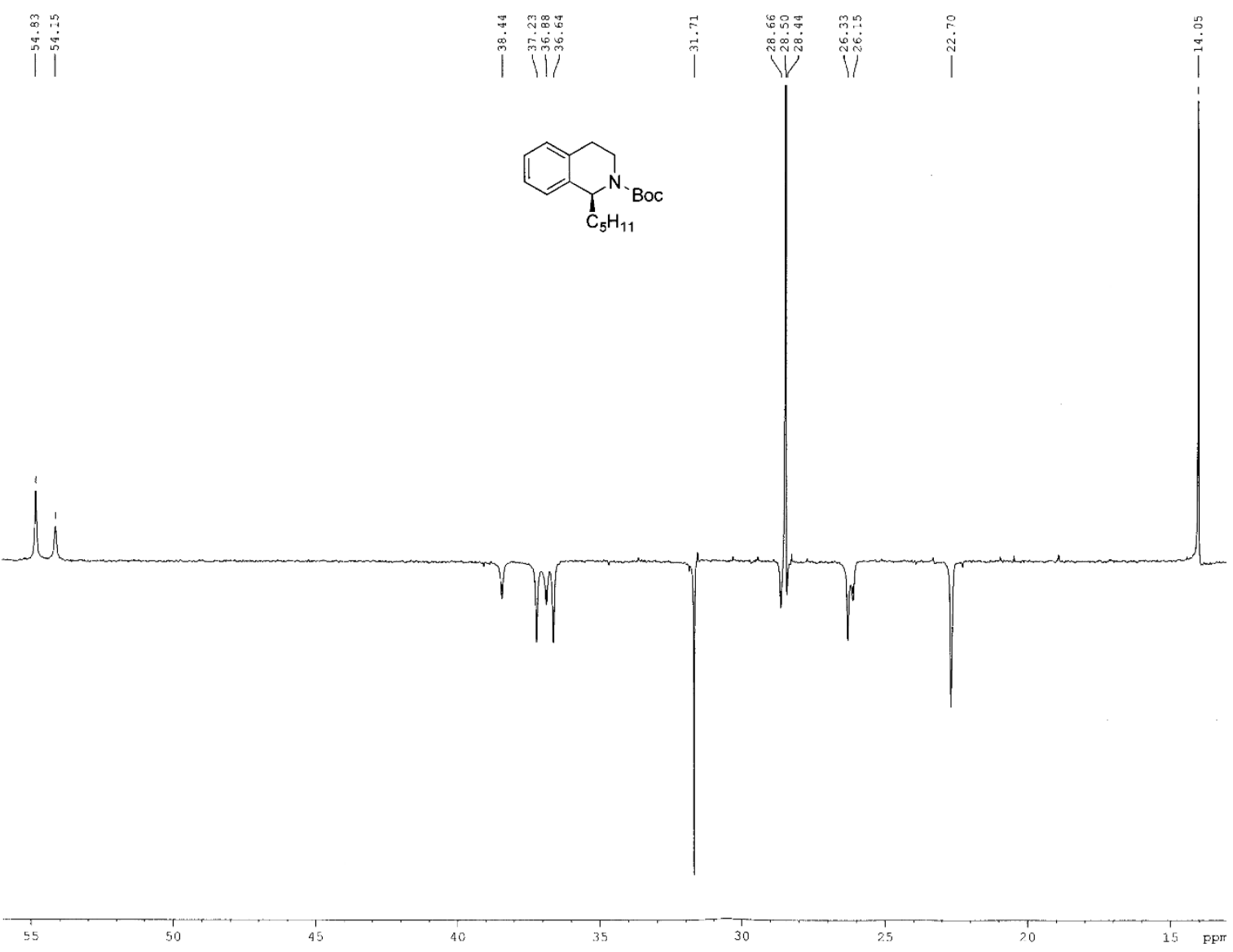

Figure $\mathbf{S}$ 122. DEPT C NMR spectrum $\left(\mathrm{CDCl}_{3}, 100 \mathrm{MHz}\right)$ of derivative (+)-10c. 
<smiles>CCCCCCCCCCC(=O)N1CCc2ccccc2C1(C)C(=O)O</smiles>

$(+)-10 d$

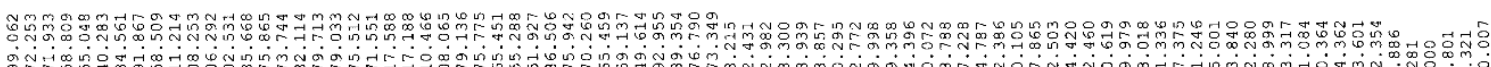

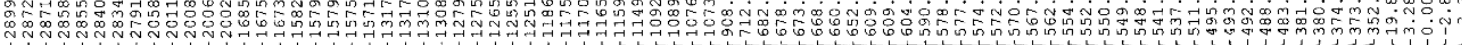

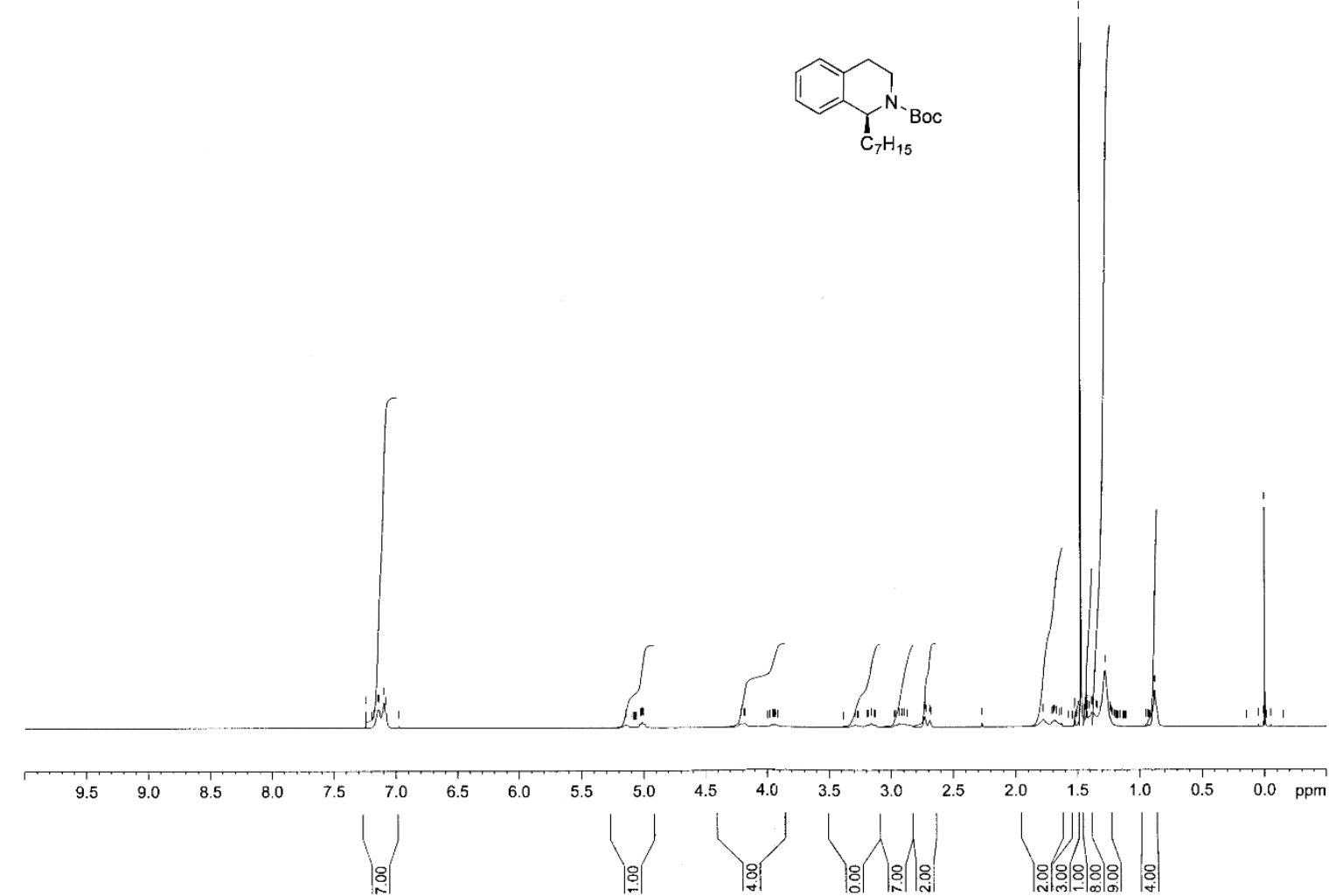

Figure S 123. ${ }^{1} \mathrm{H}$ NMR spectrum $\left(\mathrm{CDCl}_{3}, 400 \mathrm{MHz}\right)$ of derivative $(+)-\mathbf{1 0 d}$. 


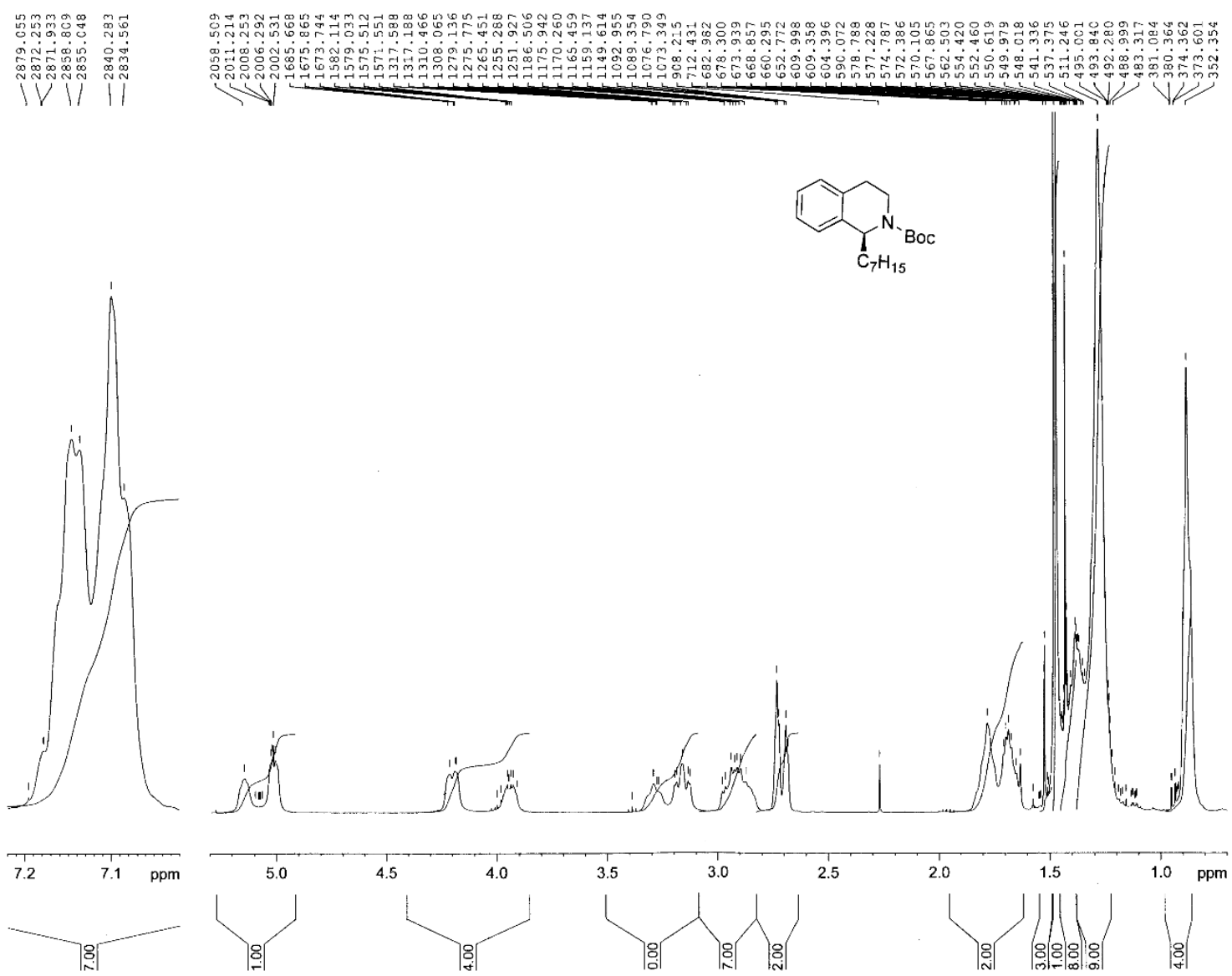

Figure S 124. ${ }^{1} \mathrm{H}$ NMR spectrum $\left(\mathrm{CDCl}_{3}, 400 \mathrm{MHz}\right)$ of derivative $(+)-10 d$. 
S127
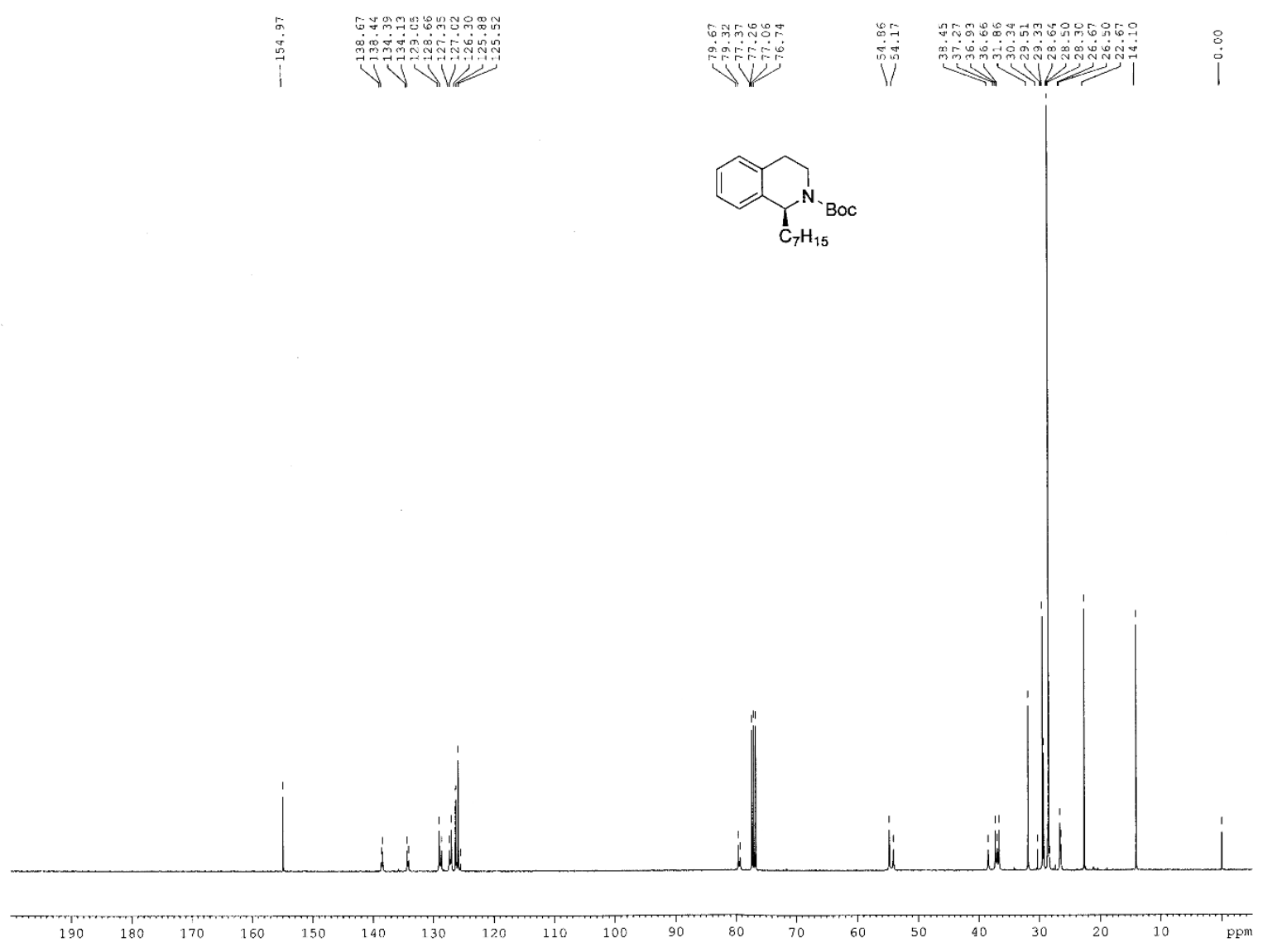

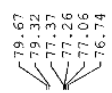

踥
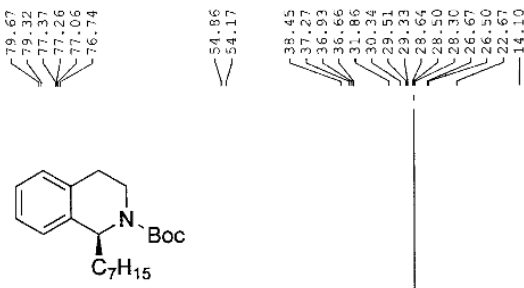

Figure S 125. ${ }^{13} \mathrm{C}$ NMR spectrum $\left(\mathrm{CDCl}_{3}, 100 \mathrm{MHz}\right)$ of derivative (+)-10d. 


\section{$\mathrm{S} 128$}

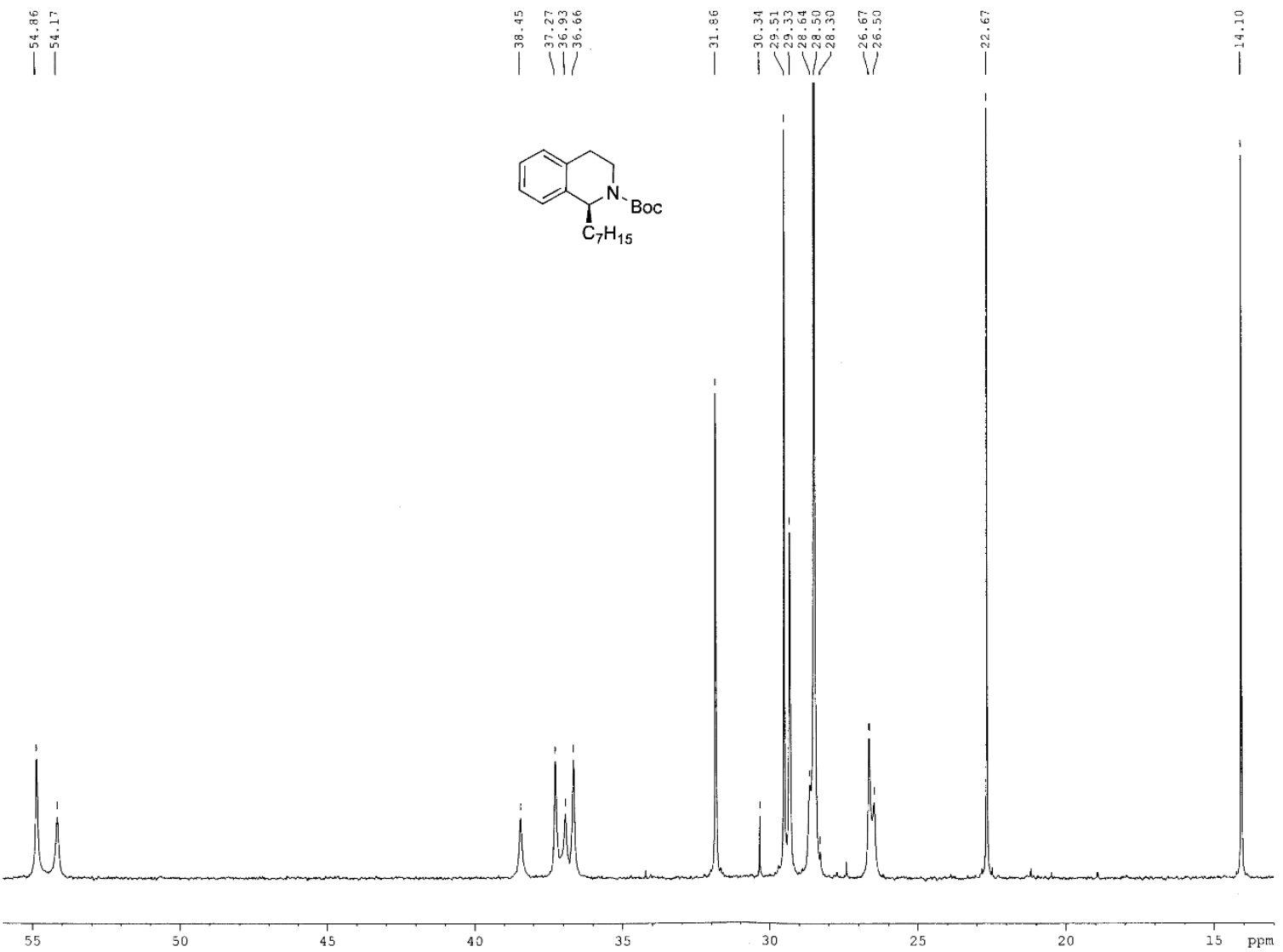

Figure S 126. ${ }^{13} \mathrm{C}$ NMR spectrum $\left(\mathrm{CDCl}_{3}, 100 \mathrm{MHz}\right)$ of derivative (+)-10d. 


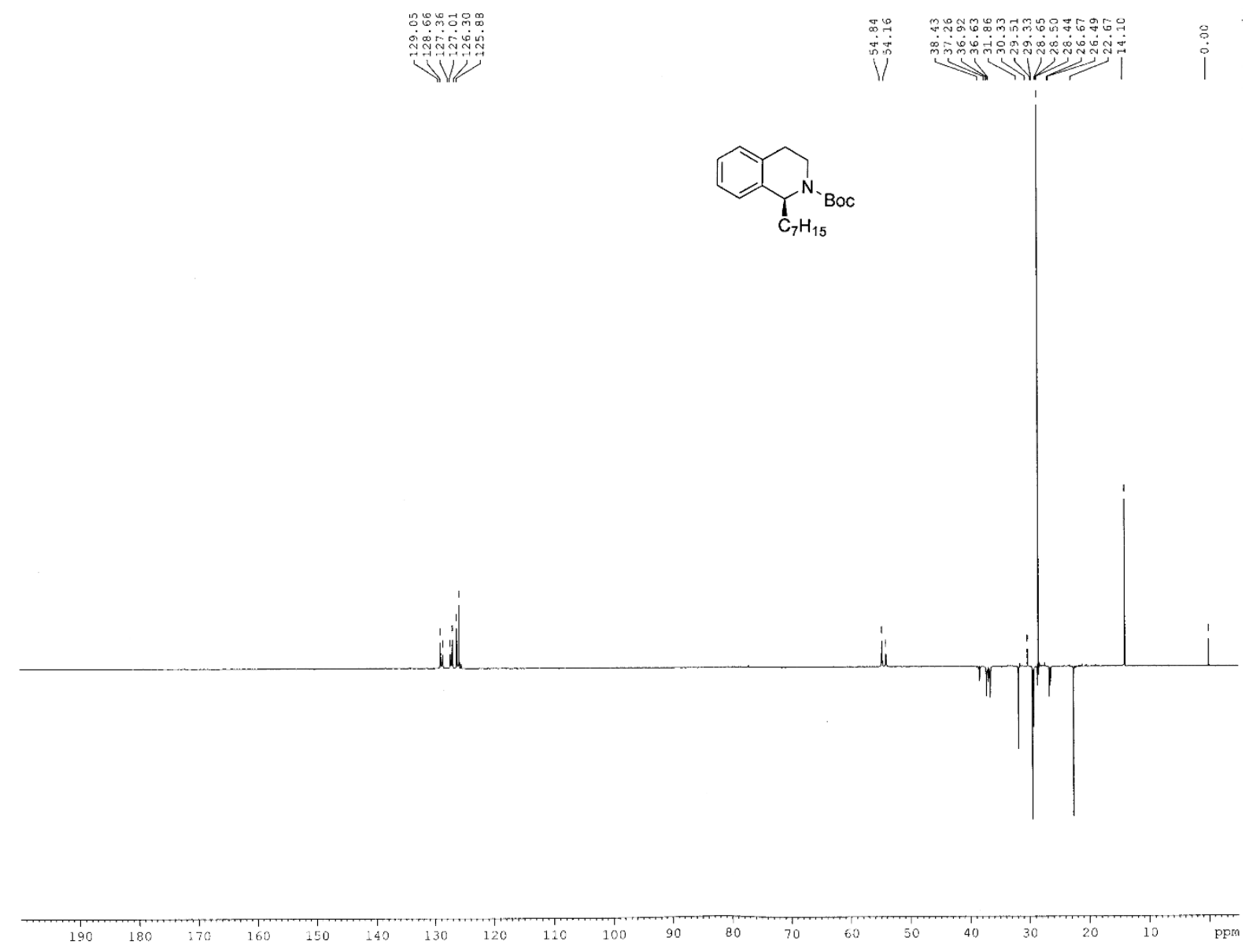

Figure $\mathrm{S}$ 127. DEPT C NMR spectrum $\left(\mathrm{CDCl}_{3}, 100 \mathrm{MHz}\right)$ of derivative (+)-10d. 


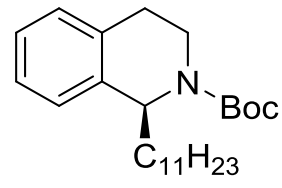

$(+)-10 e$

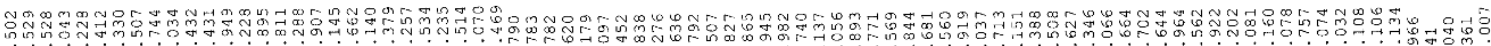

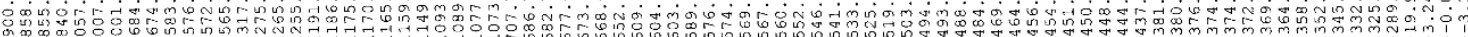

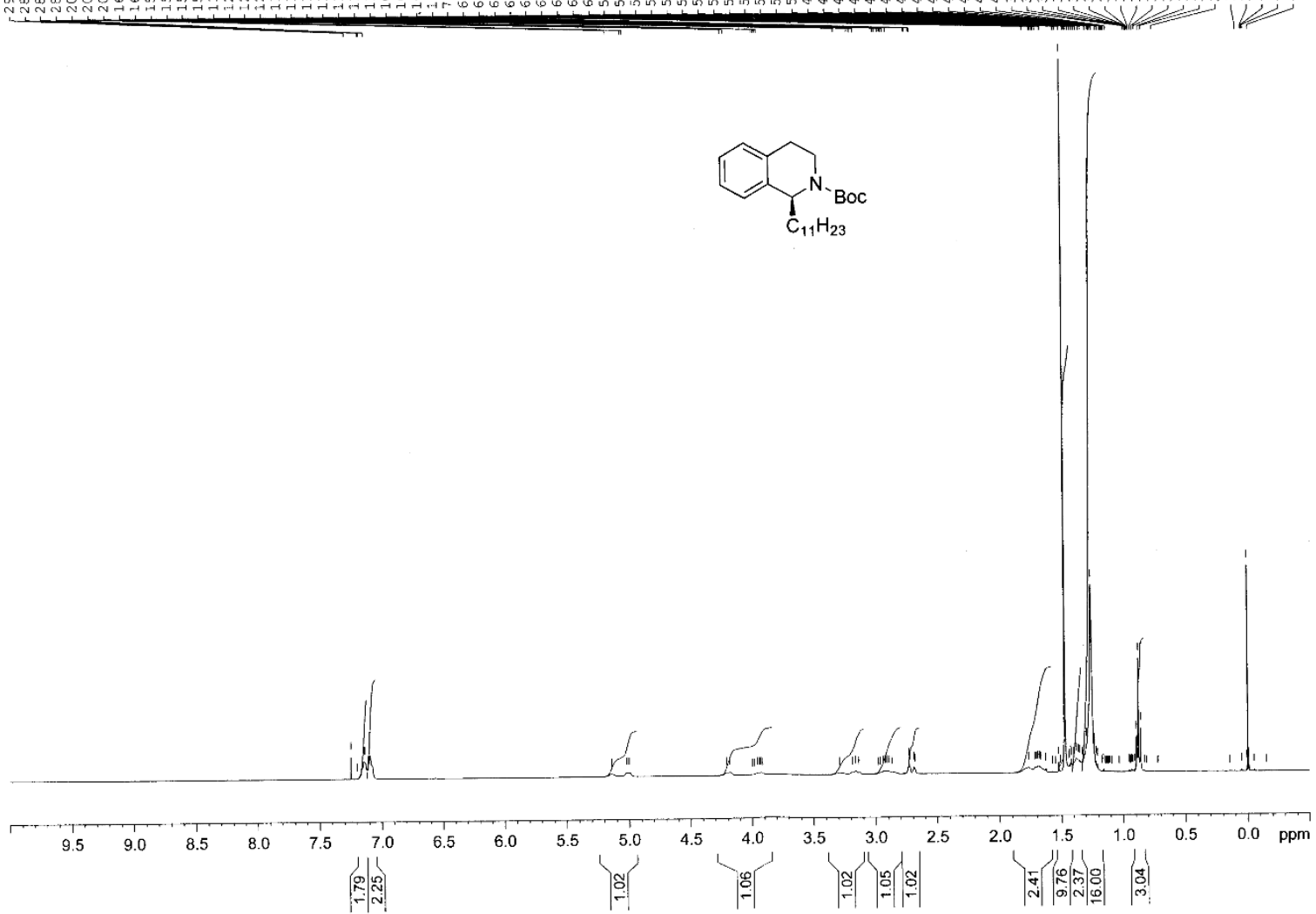

Figure S 128. ${ }^{1} \mathrm{H}$ NMR spectrum $\left(\mathrm{CDCl}_{3}, 400 \mathrm{MHz}\right)$ of derivative (+)-10e. 


\section{S131}

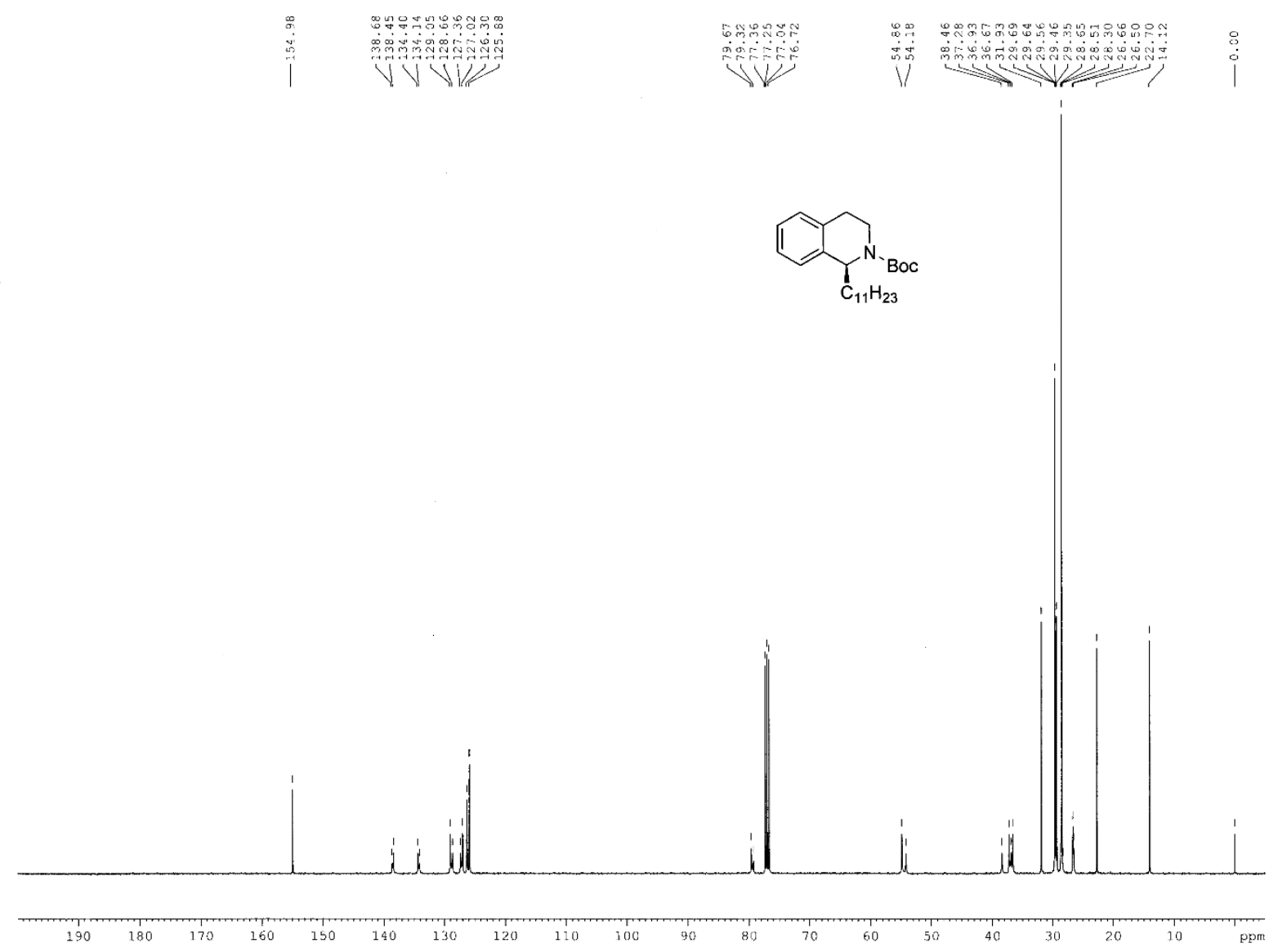

Figure S 129. ${ }^{13} \mathrm{C}$ NMR spectrum $\left(\mathrm{CDCl}_{3}, 100 \mathrm{MHz}\right)$ of derivative $(+)-\mathbf{1 0 e}$. 


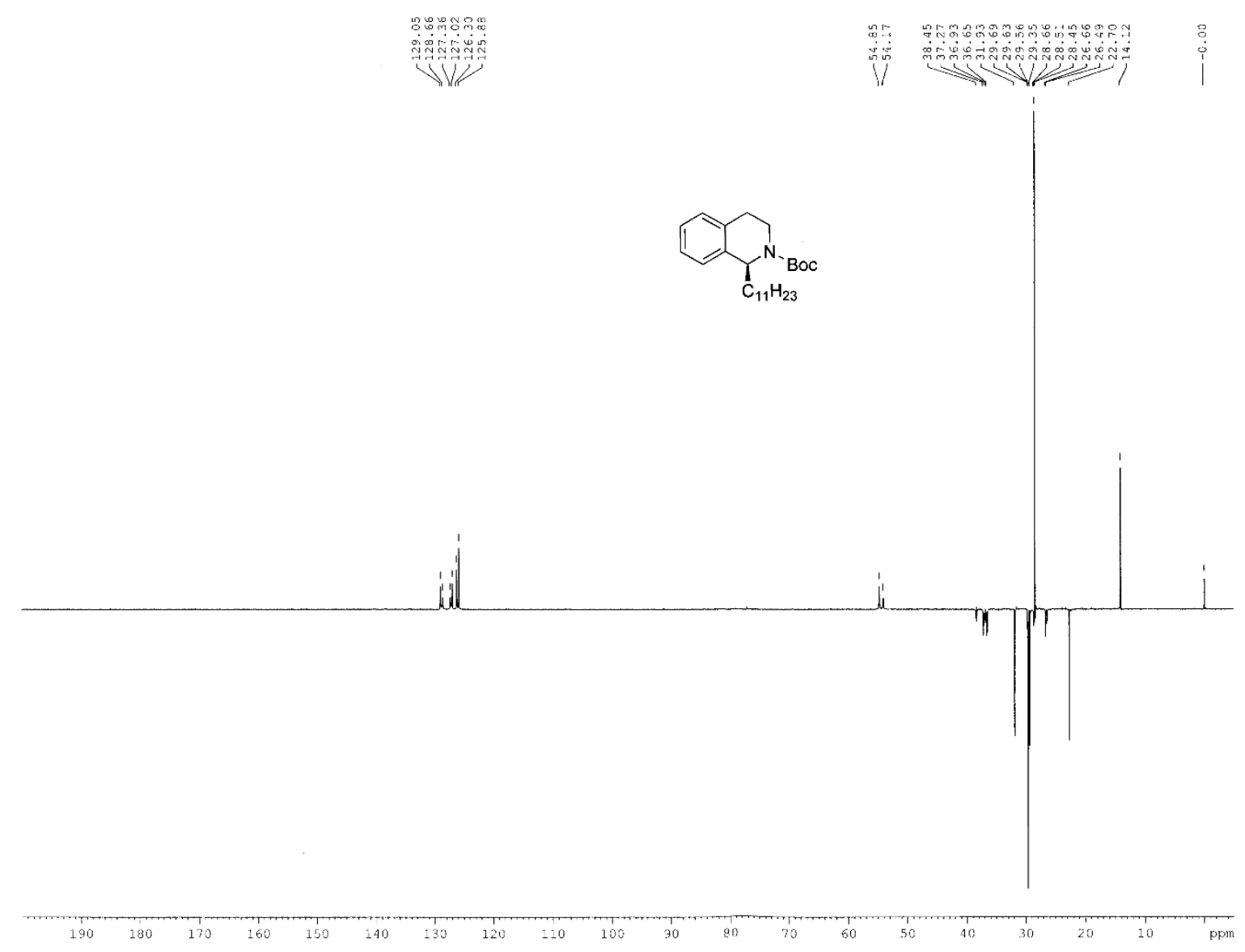

Figure S 130. DEPT C NMR spectrum $\left(\mathrm{CDCl}_{3}, 100 \mathrm{MHz}\right)$ of derivative $(+)-\mathbf{1 0 e}$. 
<smiles>COc1ccc(CC2c3ccccc3CCN2C(=O)OC(C)(C)C)cc1</smiles>

(+)-10f

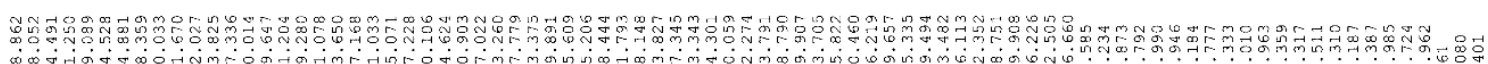

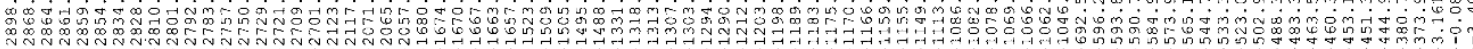

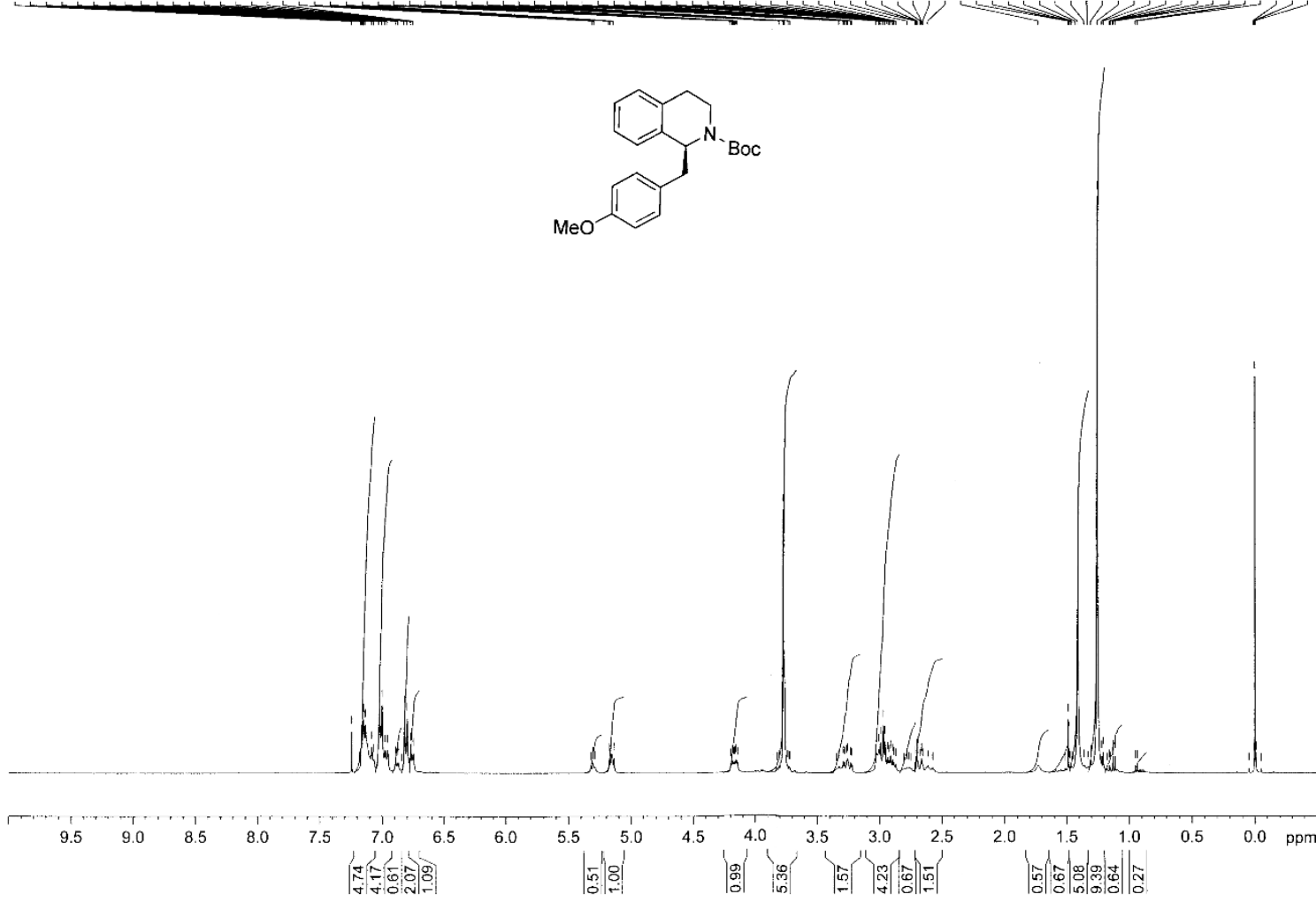

Figure $\mathrm{S}$ 131. ${ }^{1} \mathrm{H}$ NMR spectrum $\left(\mathrm{CDCl}_{3}, 400 \mathrm{MHz}\right)$ of derivative (+)-10f. 


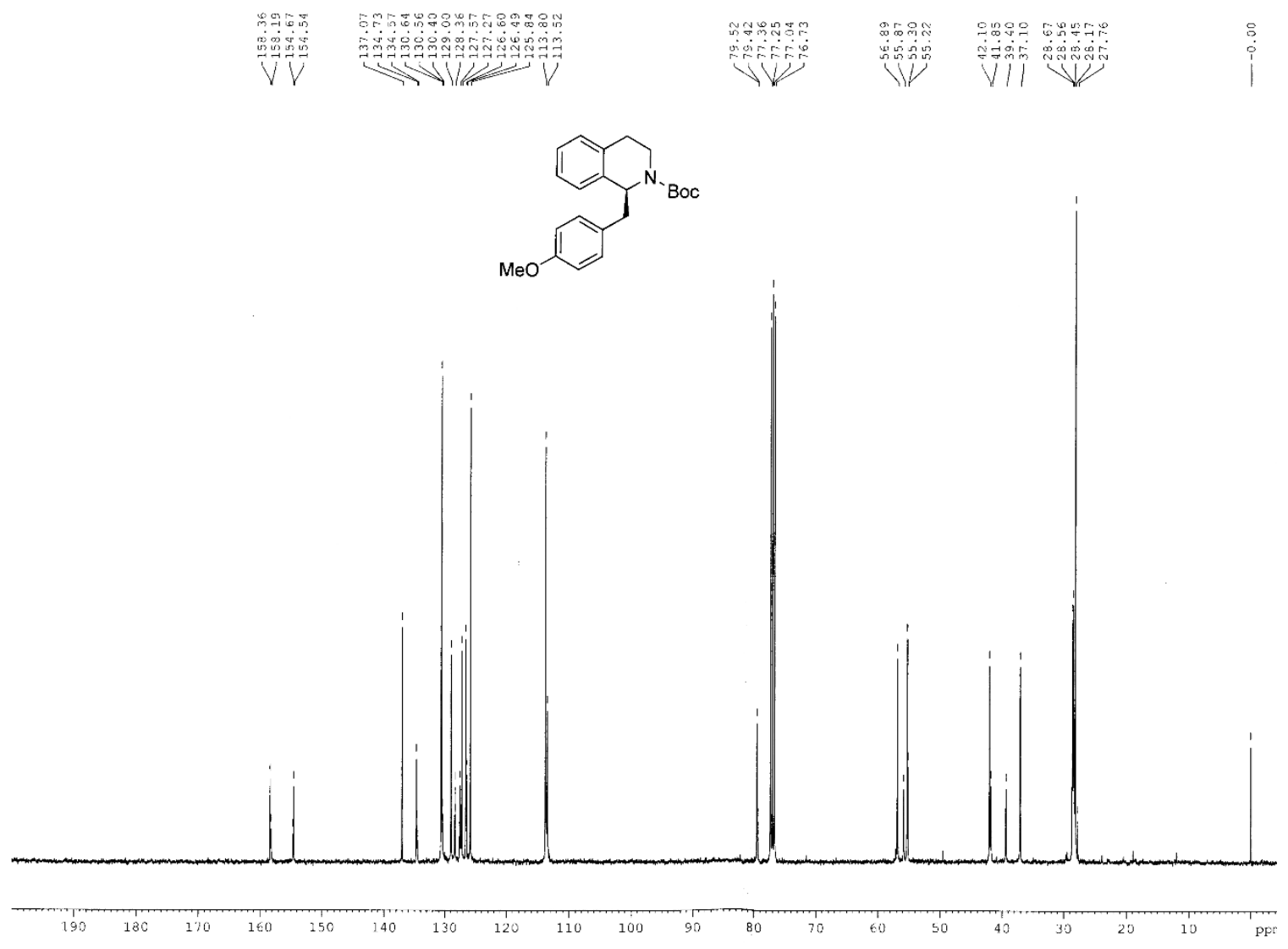

Figure S 132. ${ }^{13} \mathrm{C}$ NMR spectrum $\left(\mathrm{CDCl}_{3}, 100 \mathrm{MHz}\right)$ of derivative (+)-10f. 


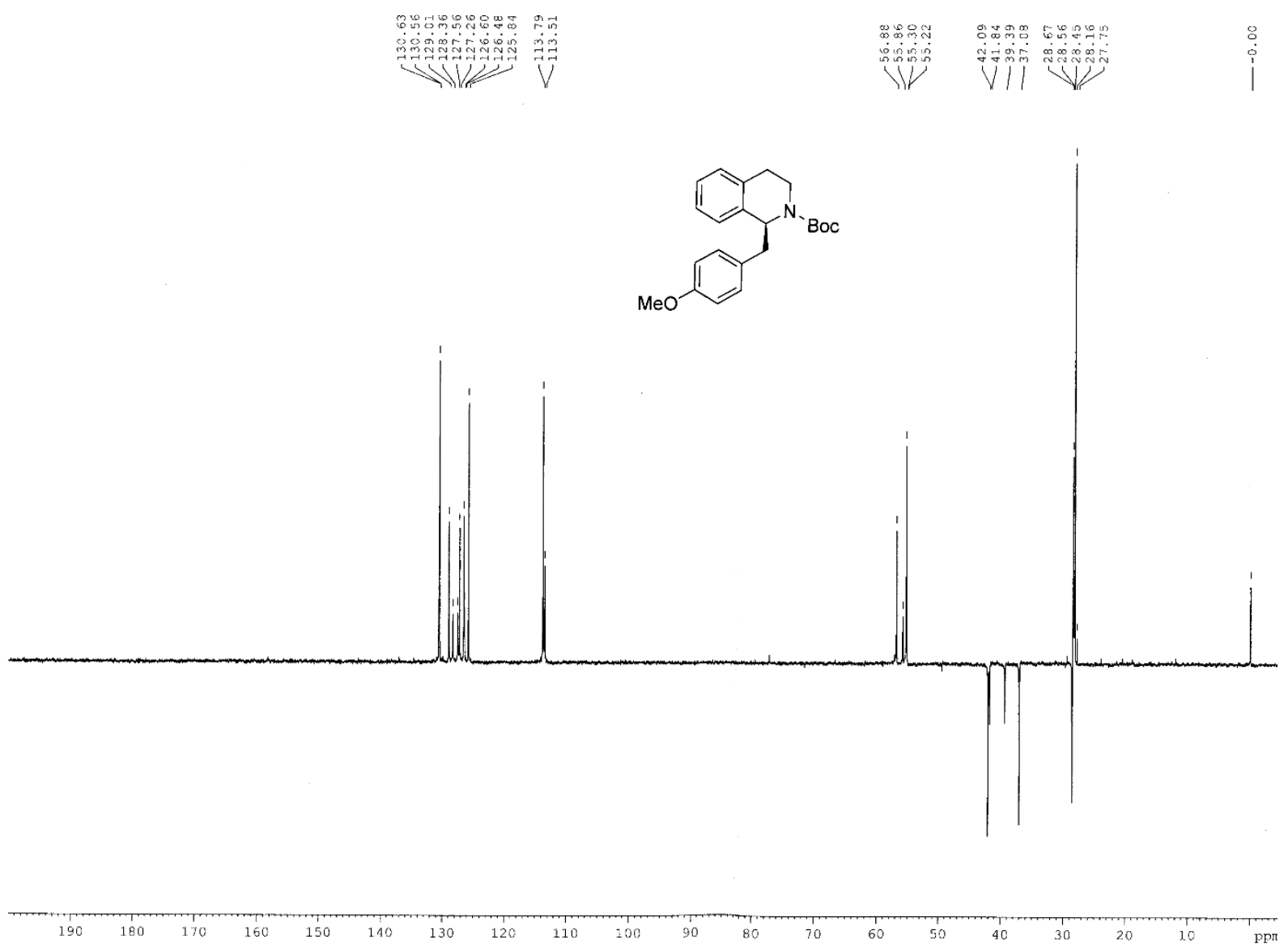

Figure S 133. DEPT C NMR spectrum ( $\left.\mathrm{CDCl}_{3}, 100 \mathrm{MHz}\right)$ of derivative (+)-10f. 
<smiles>COc1ccc(CC2c3ccccc3CCN2C(C)(C)C)cc1OC</smiles>

$(+)-10 g$<smiles>COc1ccc(CC2c3ccccc3CCN2C(C)(C)C)cc1</smiles>

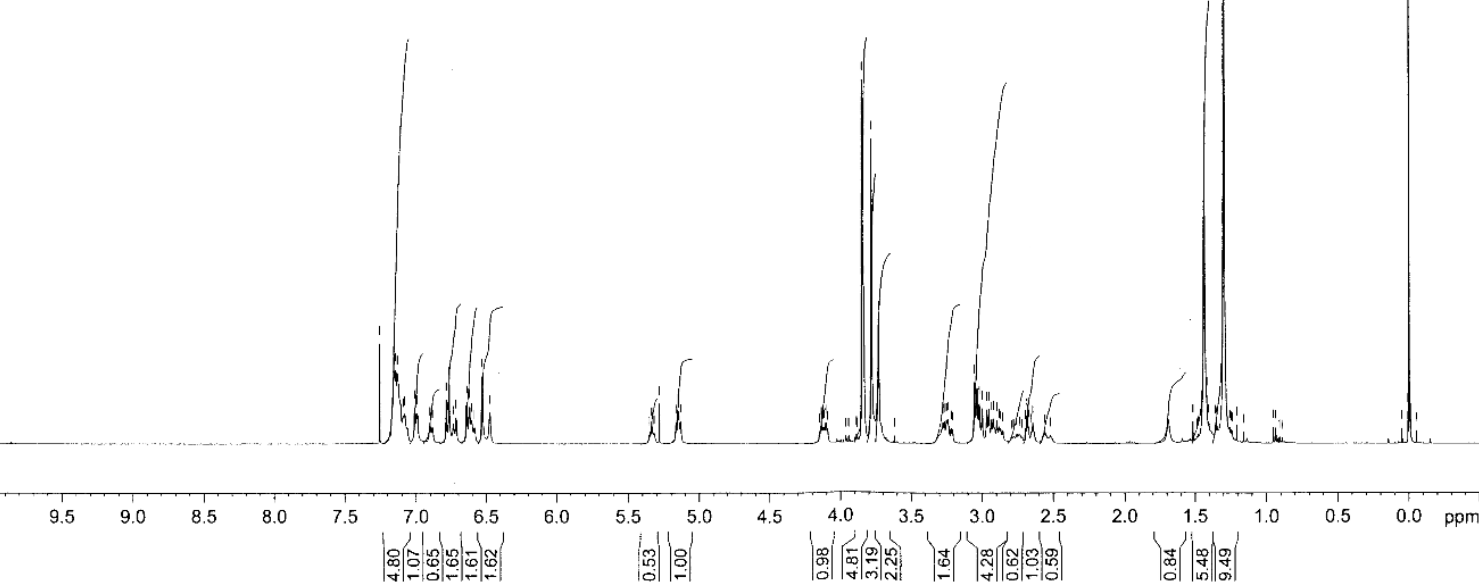

Figure S 134. ${ }^{1 \mathrm{H}} \mathrm{NMR}$ spectrum $\left(\mathrm{CDCl}_{3}, 400 \mathrm{MHz}\right)$ of derivative $(+)-\mathbf{1 0 g}$. 


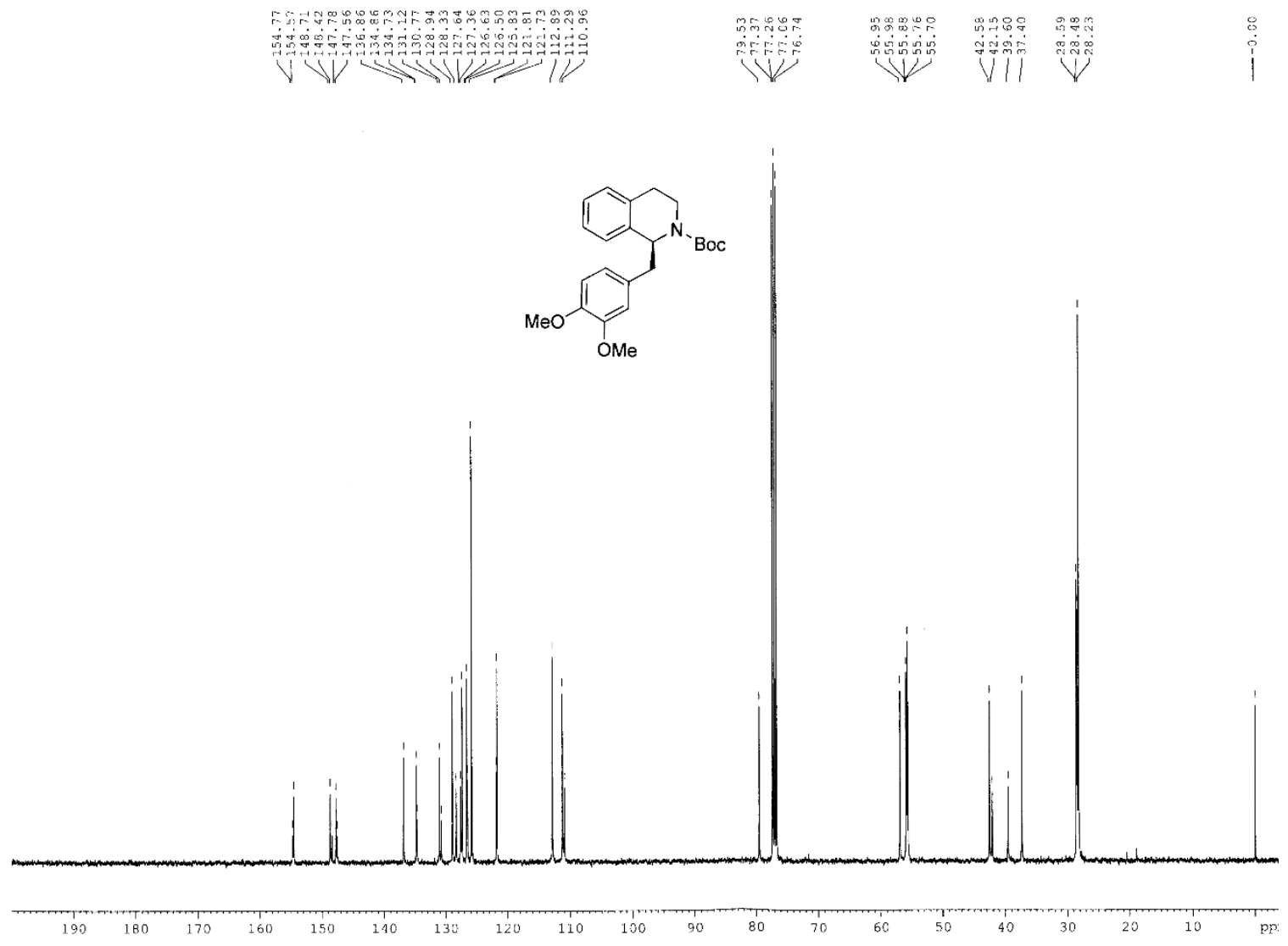

Figure S 135. ${ }^{13} \mathrm{C}$ NMR spectrum $\left(\mathrm{CDCl}_{3}, 100 \mathrm{MHz}\right)$ of derivative (+)-10g. 


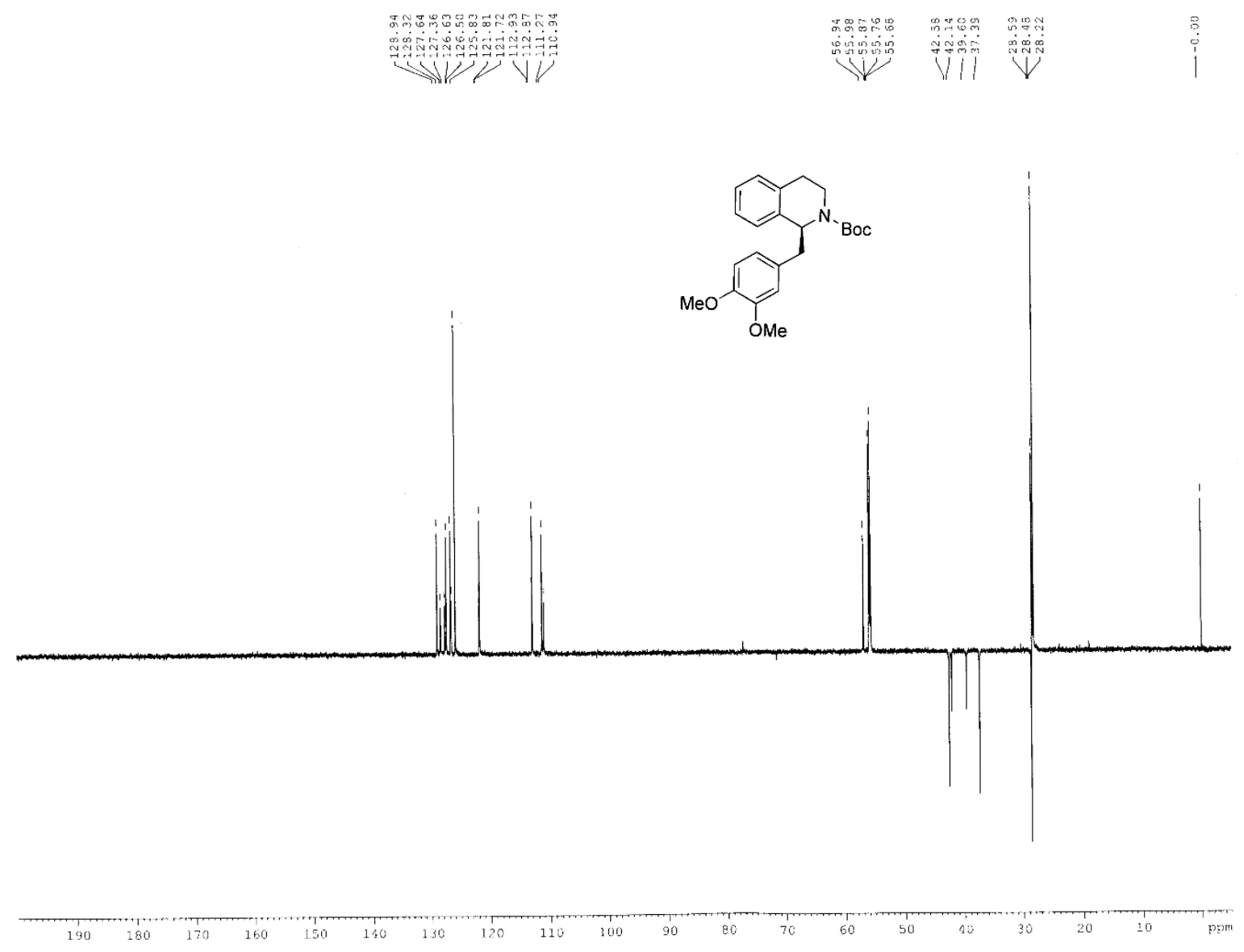

Figure S 136. DEPT C NMR spectrum $\left(\mathrm{CDCl}_{3}, 100 \mathrm{MHz}\right)$ of derivative $(+)-\mathbf{1 0 g}$. 


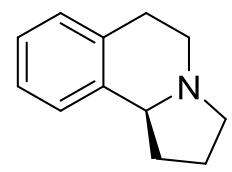

(-)-3

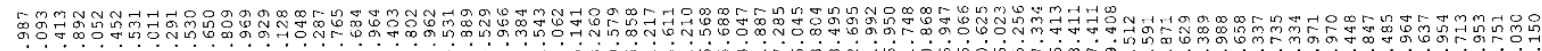

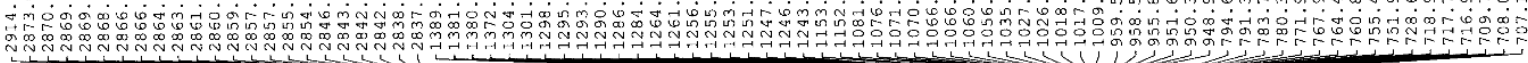

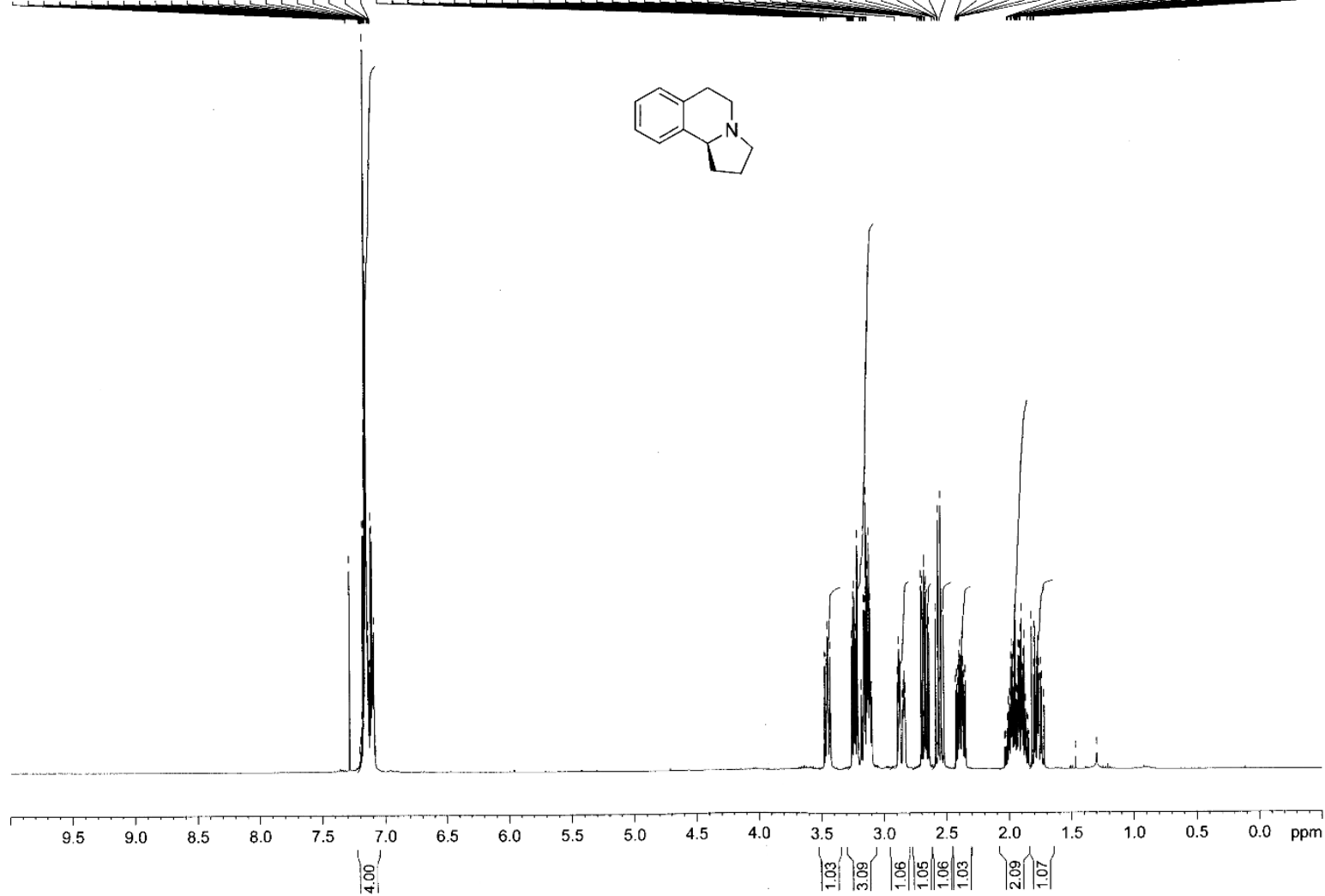

Figure S 137. ${ }^{1} \mathrm{H}$ NMR spectrum $\left(\mathrm{CDCl}_{3}, 400 \mathrm{MHz}\right)$ of derivative (-)-3 


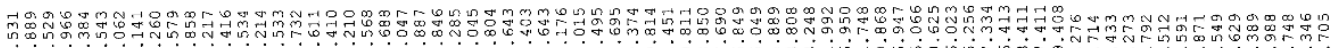

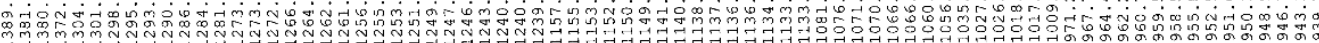
Vî<smiles>c1ccc2c(c1)CCN1CCCC21</smiles>

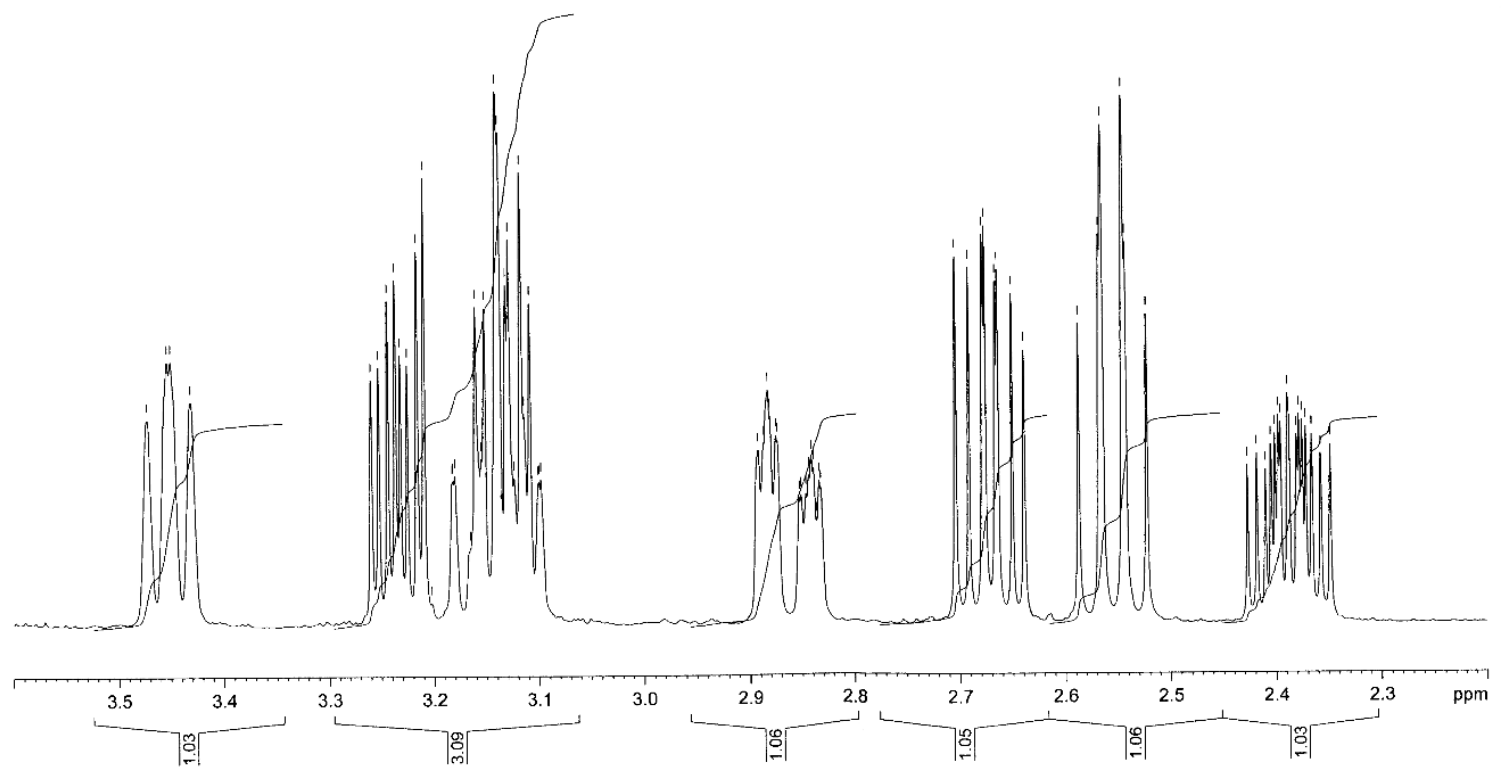

Figure S 138. ${ }^{1} \mathrm{H}$ NMR spectrum $\left(\mathrm{CDCl}_{3}, 400 \mathrm{MHz}\right)$ of derivative (-)-3. 


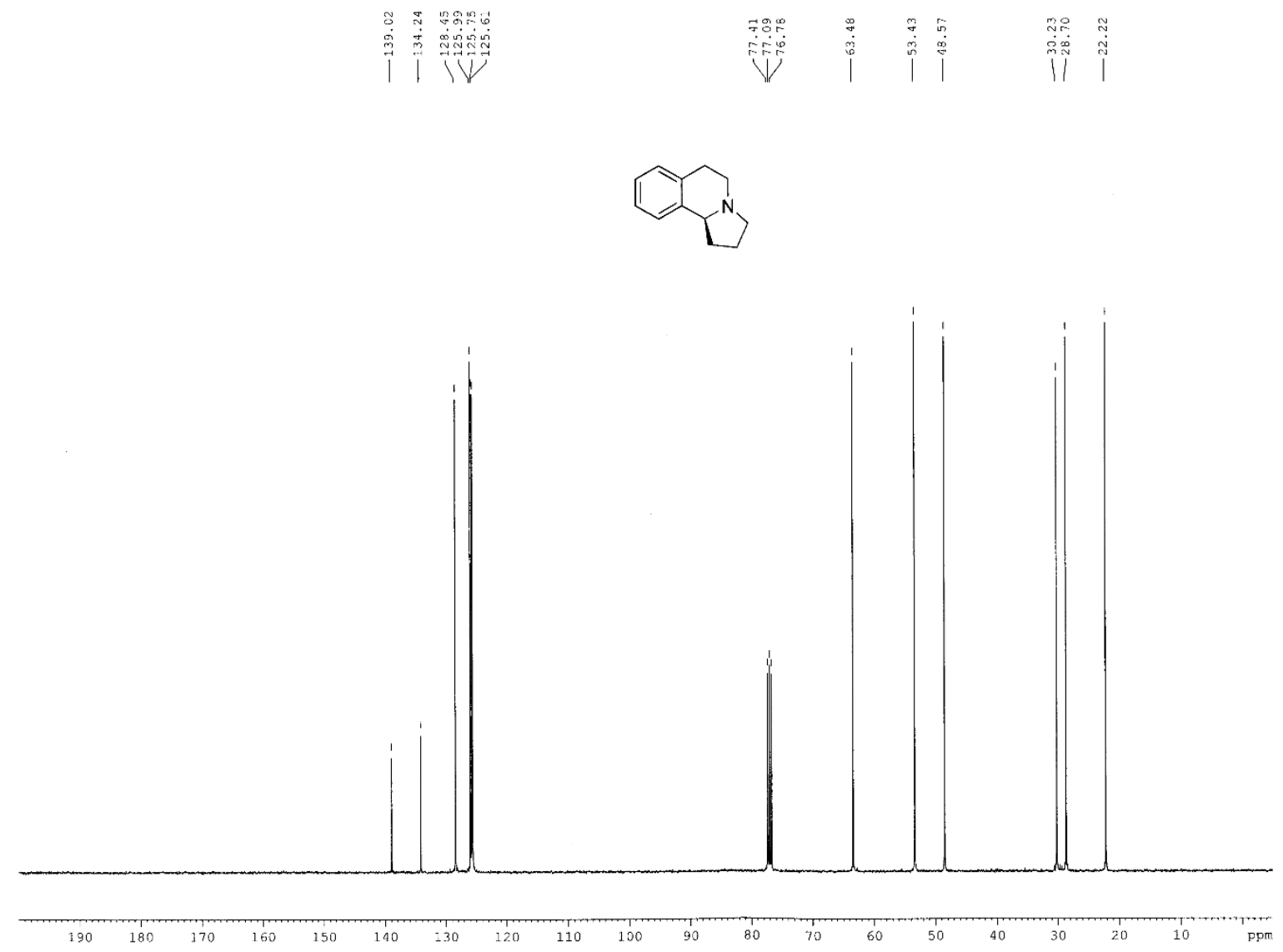

Figure S 139. ${ }^{13} \mathrm{C}$ NMR spectrum $\left(\mathrm{CDCl}_{3}, 100 \mathrm{MHz}\right)$ of derivative (-)-3. 
<smiles>c1ccc2c(c1)CCN1CCCC21</smiles>

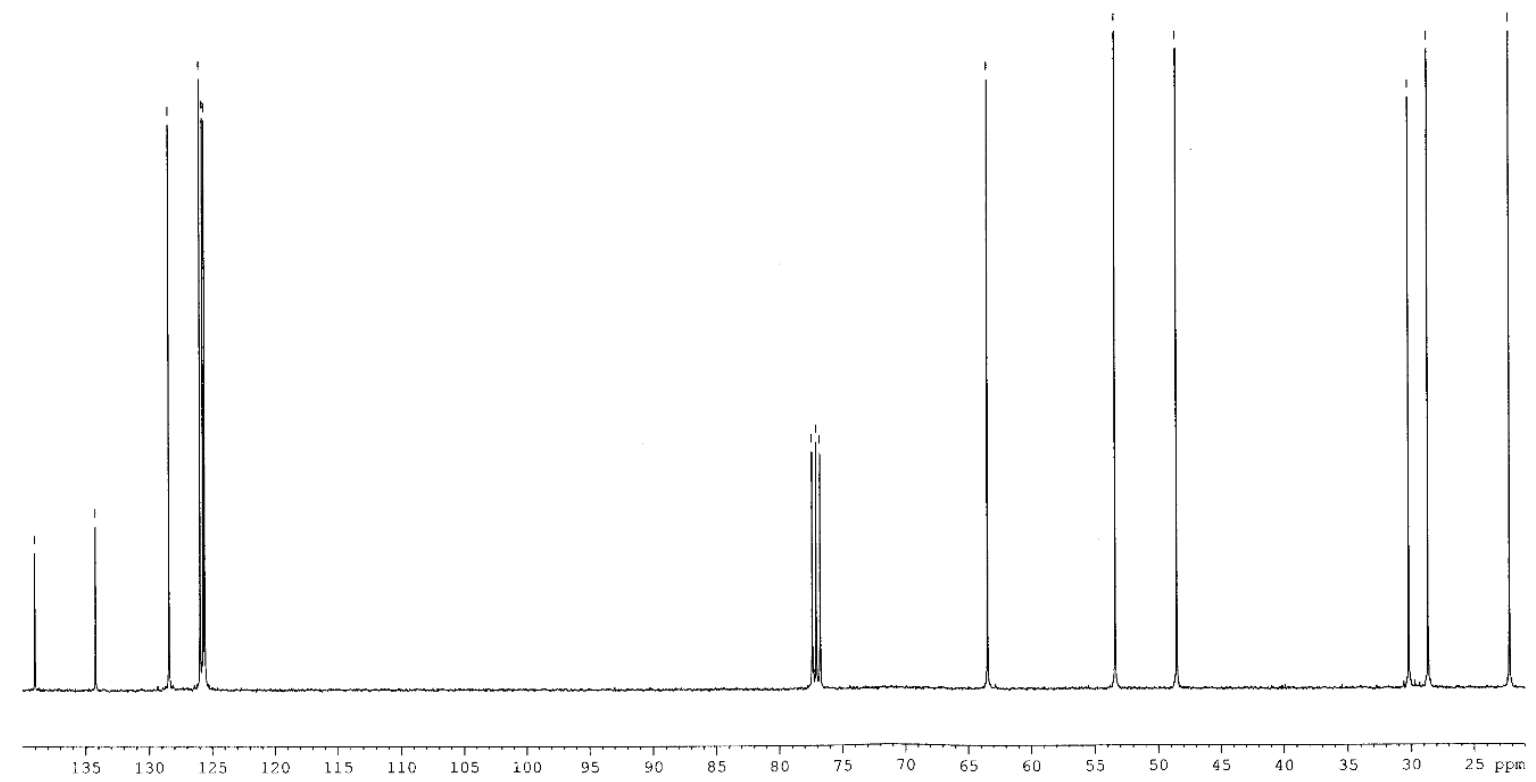

Figure S $140 .{ }^{13} \mathrm{C}$ NMR spectrum $\left(\mathrm{CDCl}_{3}, 100 \mathrm{MHz}\right)$ of derivative (-)-3. 


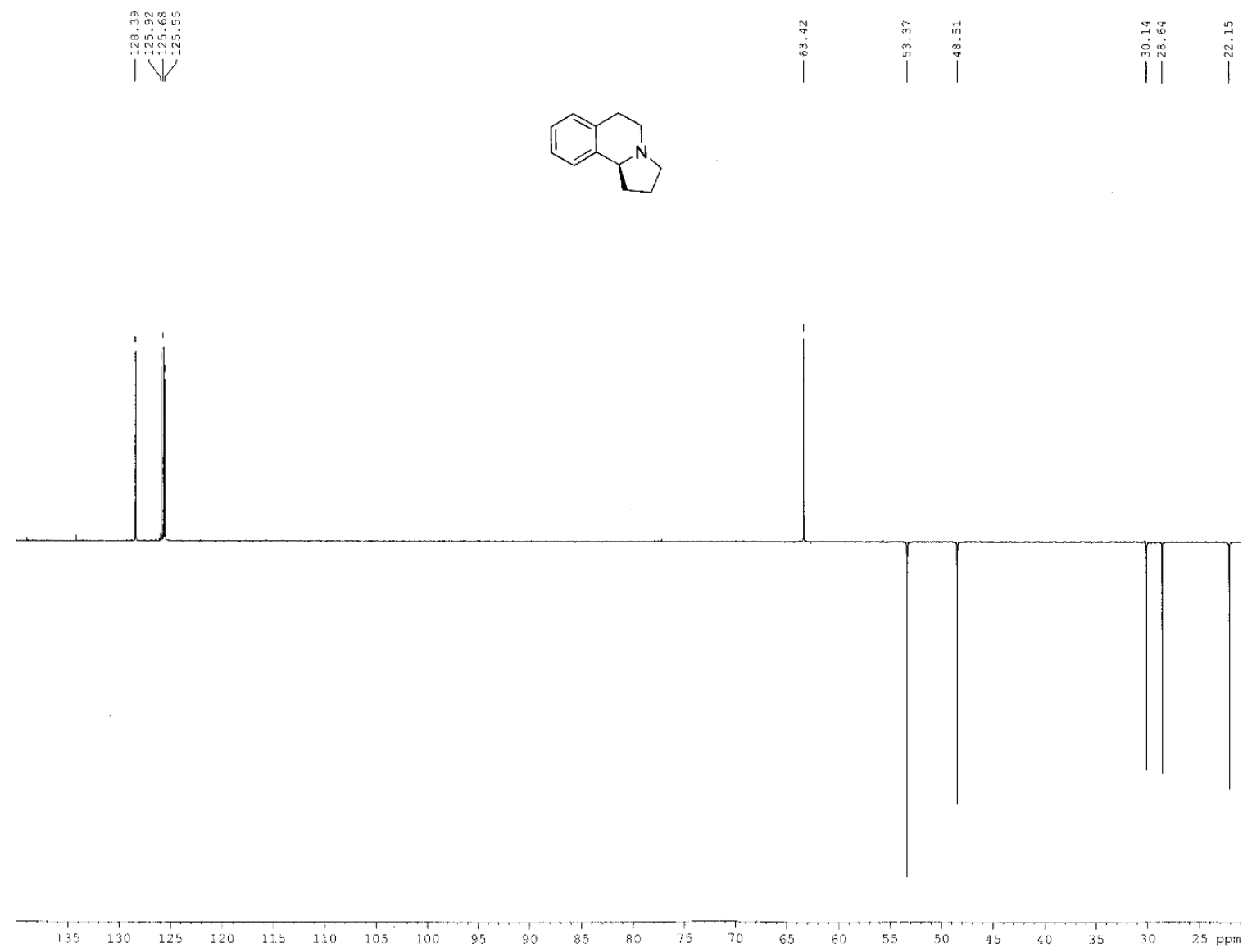

Figure S 141. DEPT C NMR spectrum ( $\left.\mathrm{CDCl}_{3}, 100 \mathrm{MHz}\right)$ of derivative (-)-3. 


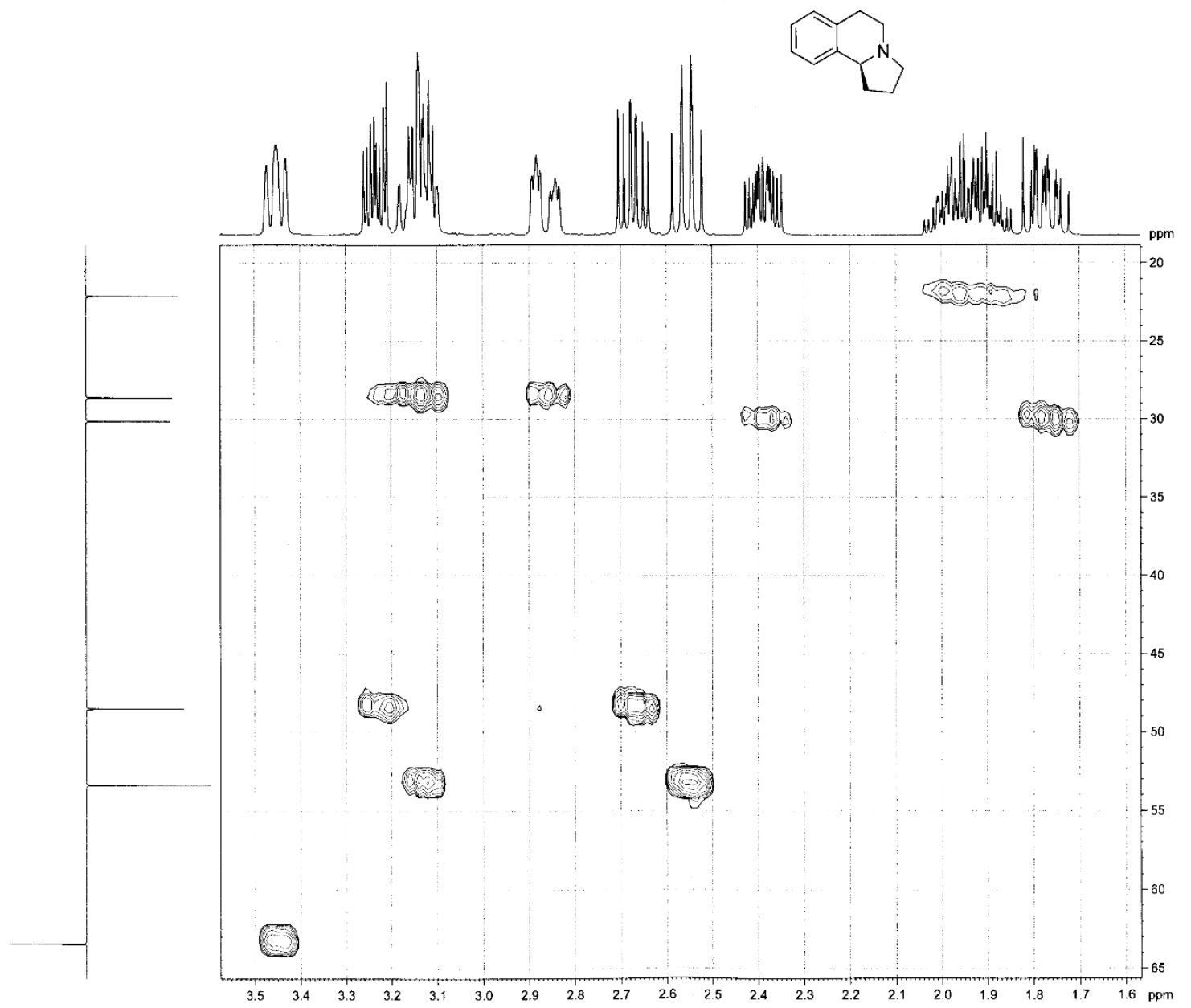

Figure $\mathbf{S}$ 142. $\mathrm{HMQC}$ spectrum $\left(\mathrm{CDCl}_{3}\right)$ of derivative (-)-3. 


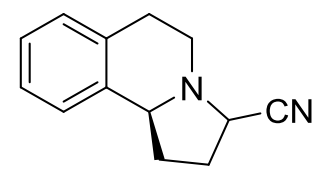

11

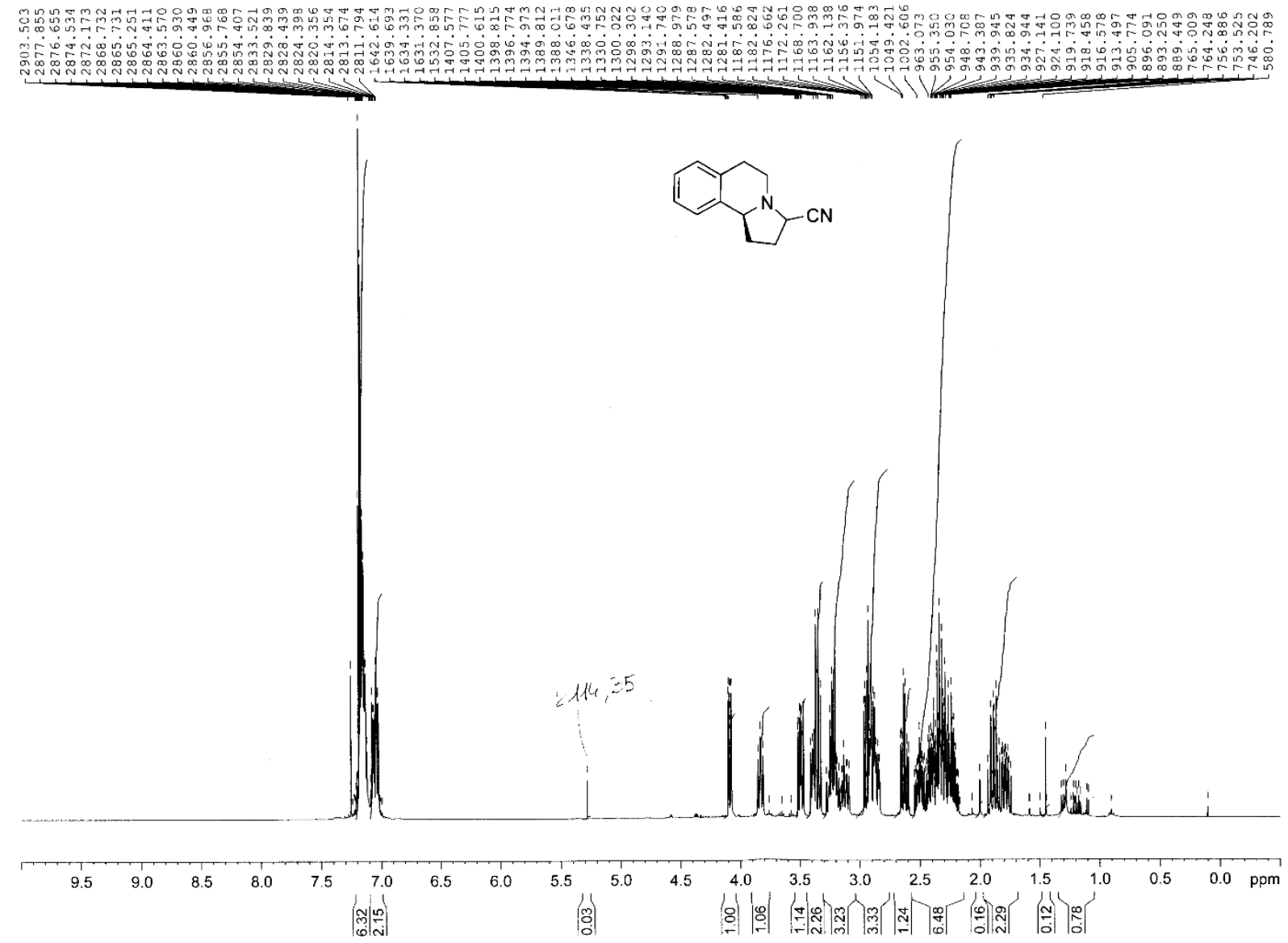

Figure S 143. ${ }^{1} \mathrm{H}$ NMR spectrum $\left(\mathrm{CDCl}_{3}, 400 \mathrm{MHz}\right)$ of derivative 11. 


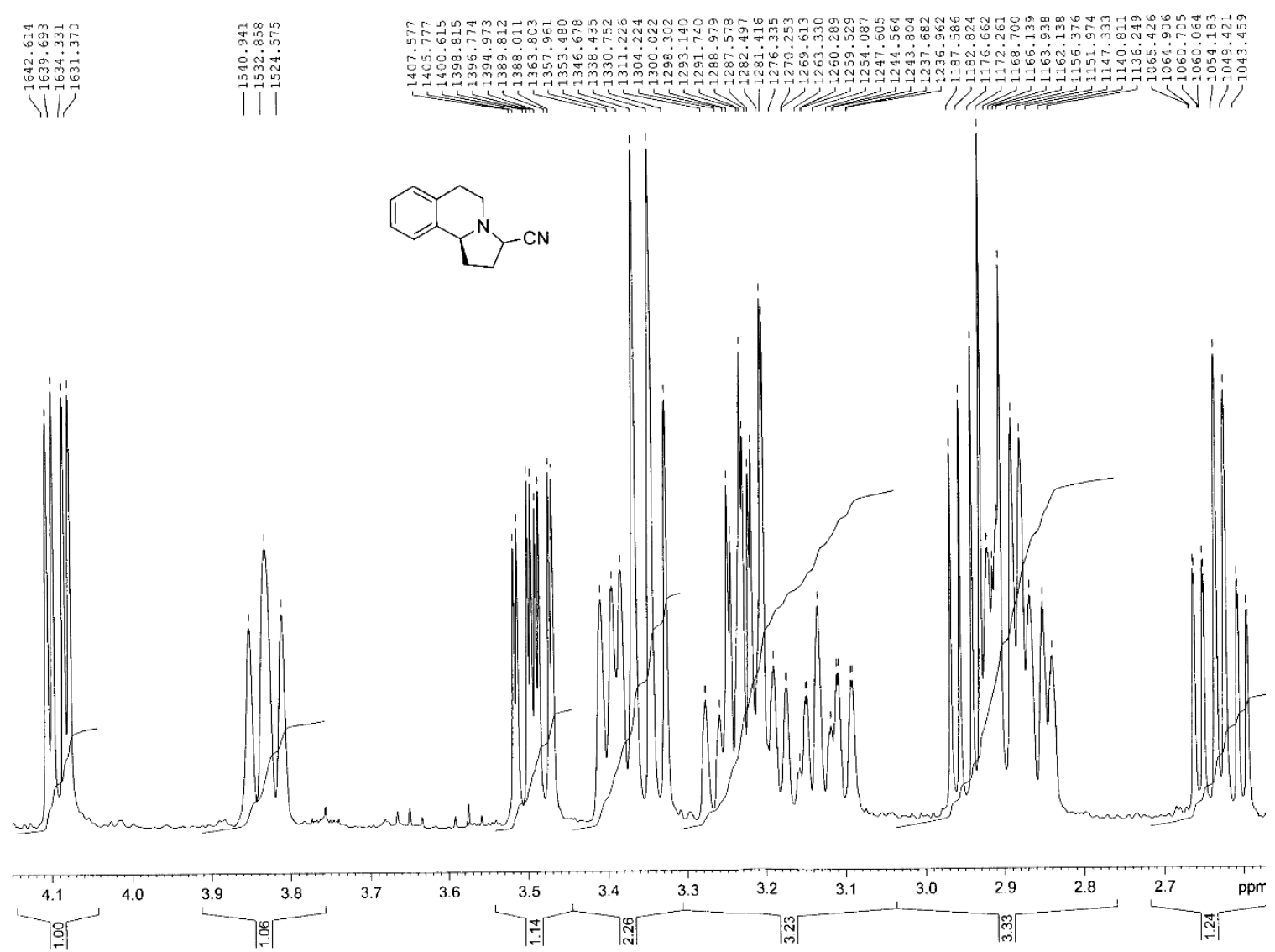

Figure S 144. ${ }^{1} \mathrm{H}$ NMR spectrum $\left(\mathrm{CDCl}_{3}, 400 \mathrm{MHz}\right)$ of derivative 11. 


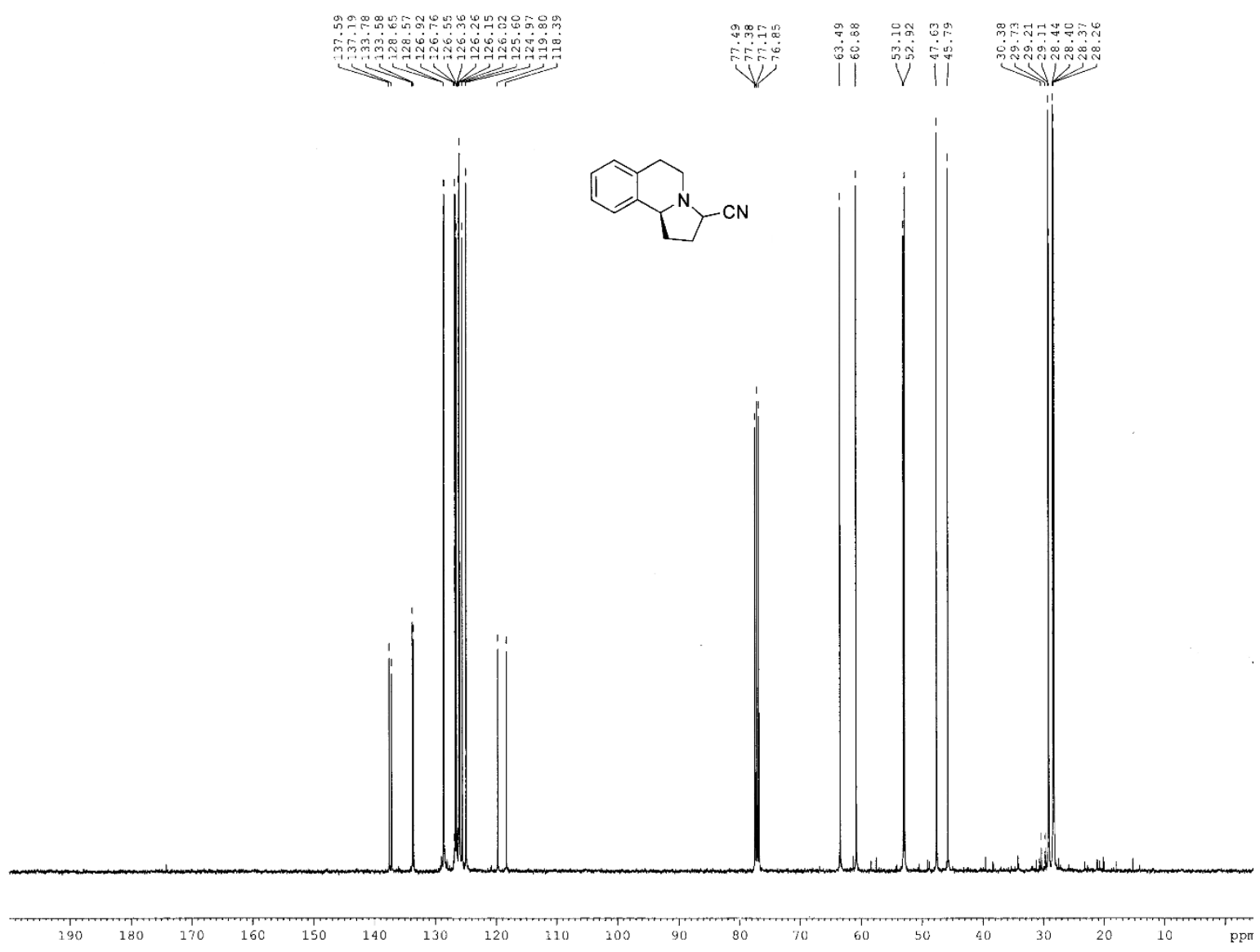

Figure S 145. ${ }^{13} \mathrm{C}$ NMR spectrum $\left(\mathrm{CDCl}_{3}, 100 \mathrm{MHz}\right)$ of derivative 11. 


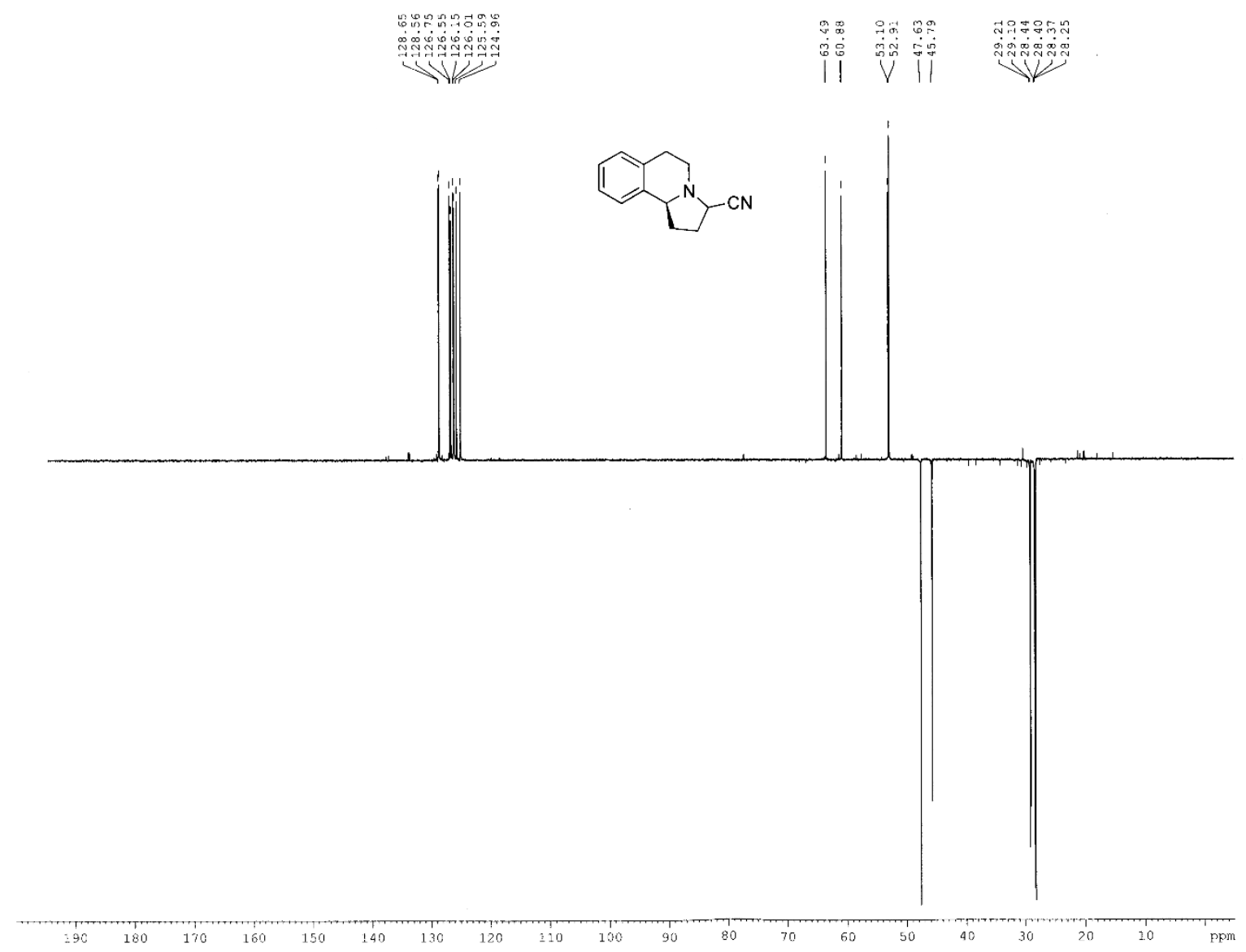

Figure S 146. DEPT C NMR spectrum $\left(\mathrm{CDCl}_{3}, 100 \mathrm{MHz}\right)$ of derivative 11. 


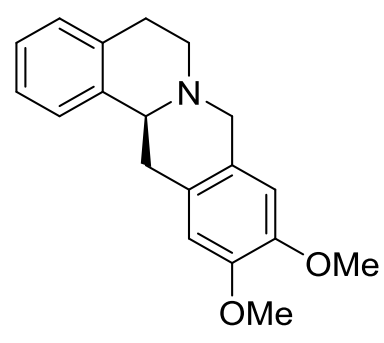

(-) -4
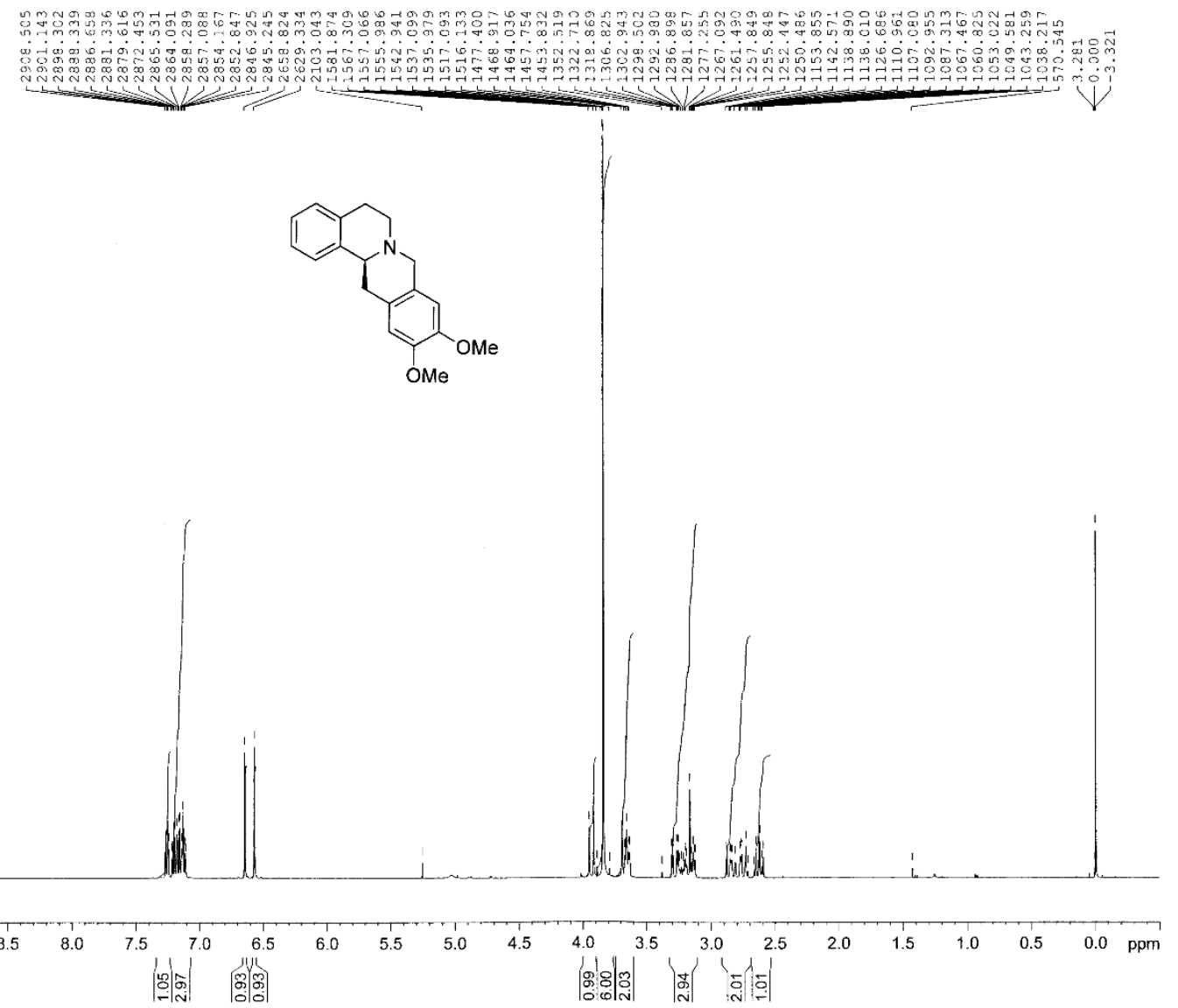

Figure S 147. ${ }^{1} \mathrm{H} \mathrm{NMR}$ spectrum $\left(\mathrm{CDCl}_{3}, 400 \mathrm{MHz}\right)$ of derivative (-)-4. 


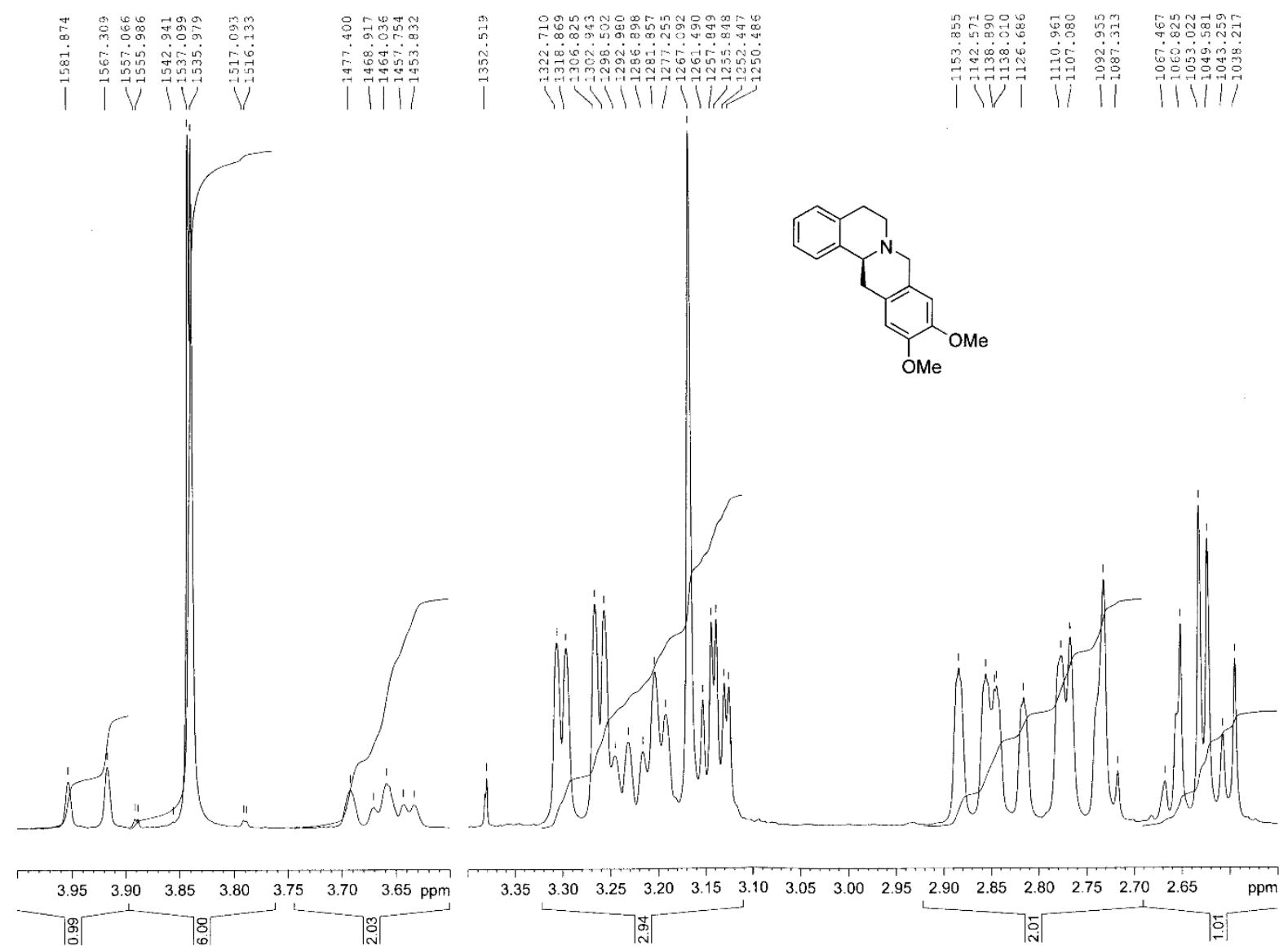

Figure $\mathbf{S}$ 148. ${ }^{1} \mathrm{H}$ NMR spectrum $\left(\mathrm{CDCl}_{3}, 400 \mathrm{MHz}\right)$ of derivative (-)-4. 


\section{S151}

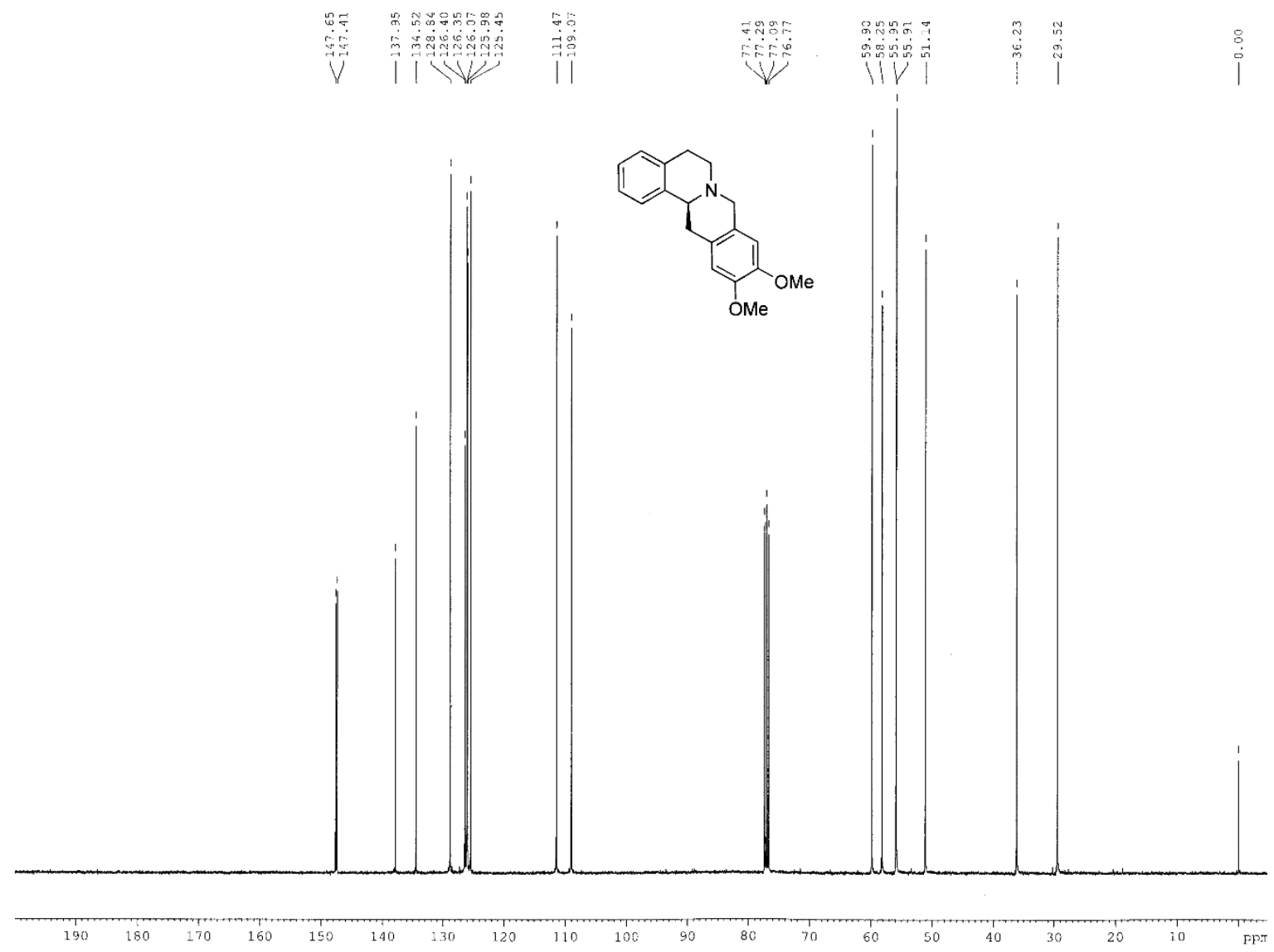

Figure $\mathbf{S}$ 149. ${ }^{13} \mathrm{C}$ NMR spectrum $\left(\mathrm{CDCl}_{3}, 100 \mathrm{MHz}\right)$ of derivative (-)-4. 
S152

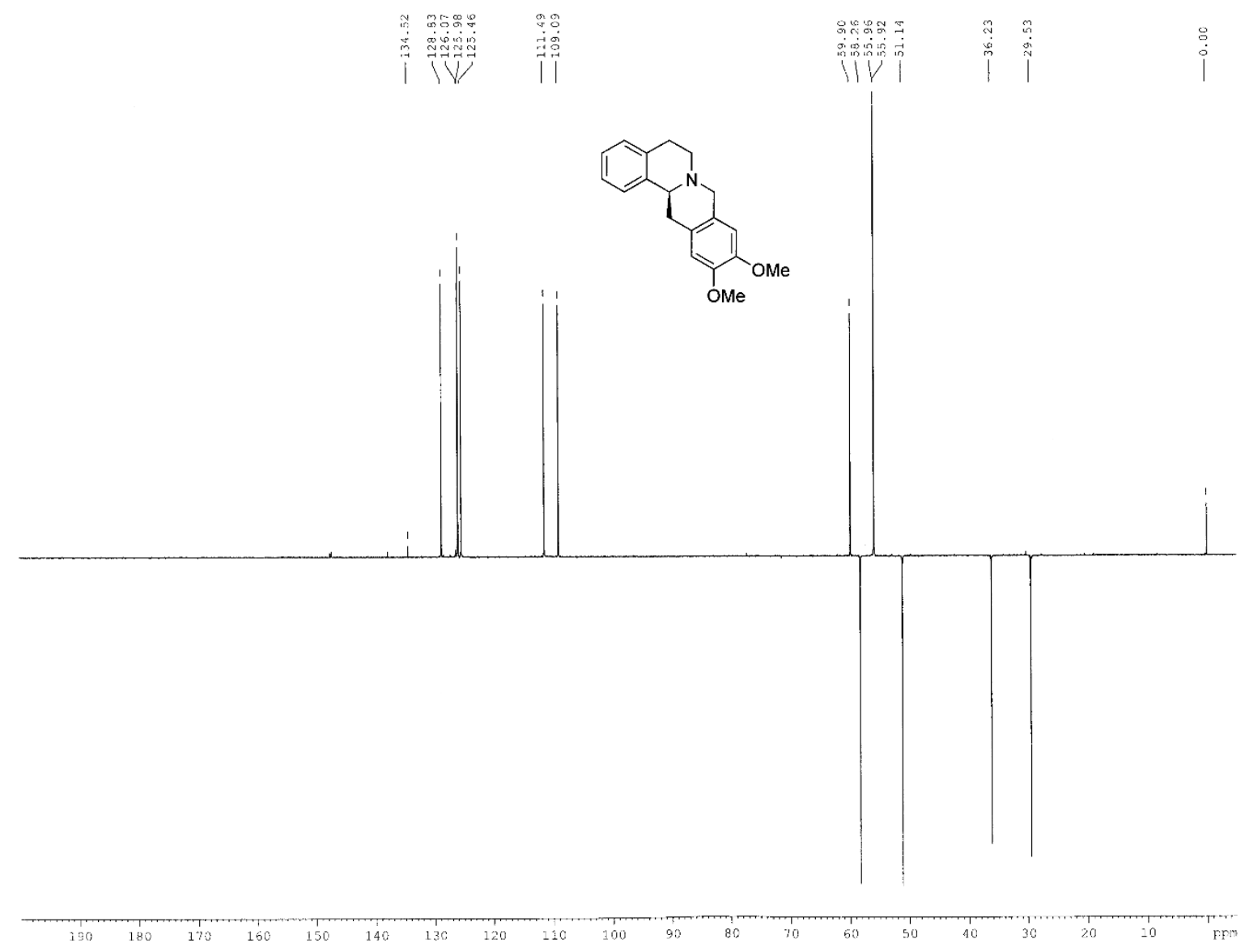

Figure S 150. DEPT C NMR spectrum $\left(\mathrm{CDCl}_{3}, 100 \mathrm{MHz}\right)$ of derivative (-)-4. 
S153

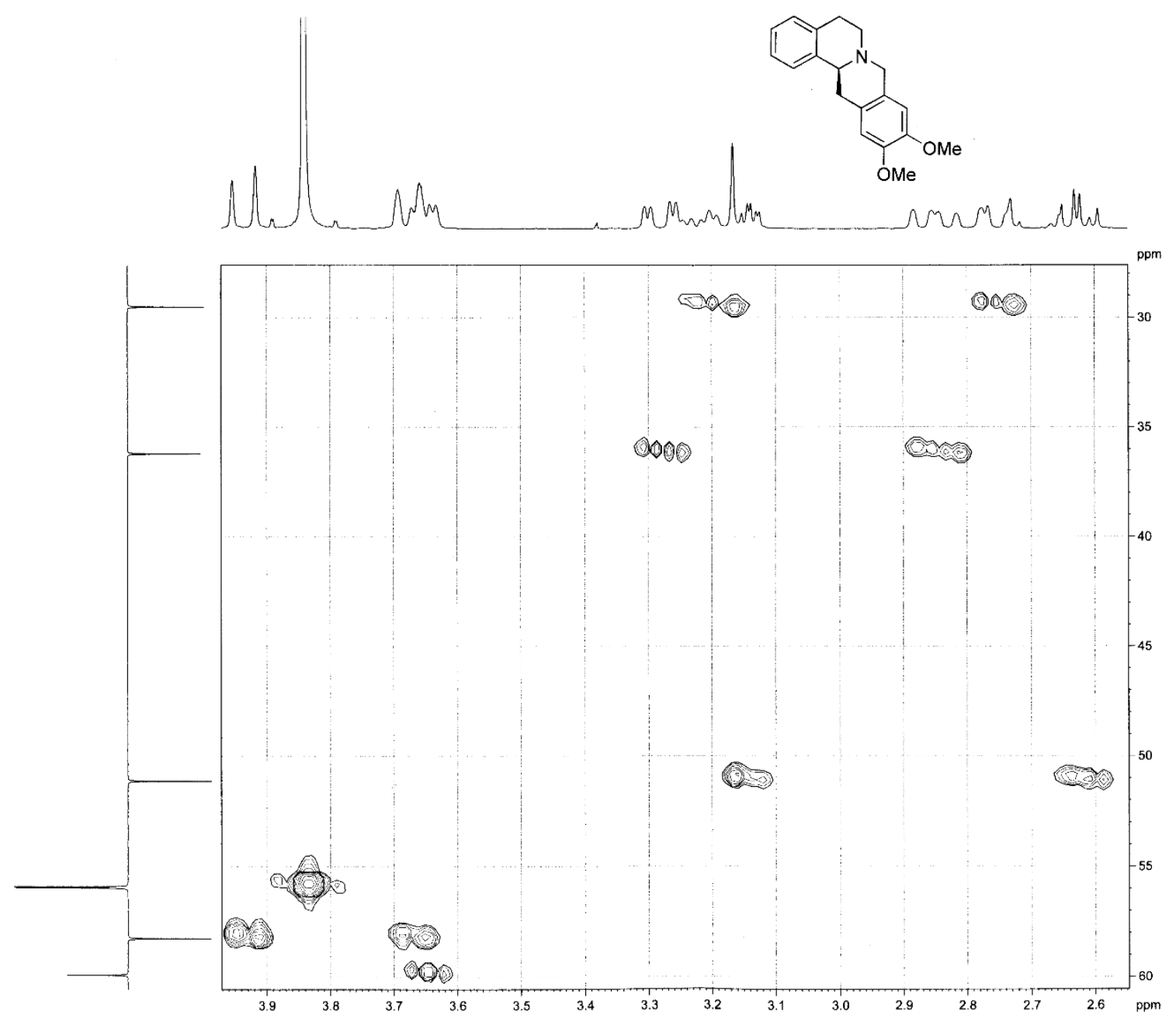

Figure S 151. $\mathrm{HMQC}$ spectrum $\left(\mathrm{CDCl}_{3}\right)$ of derivative (-)-4. 


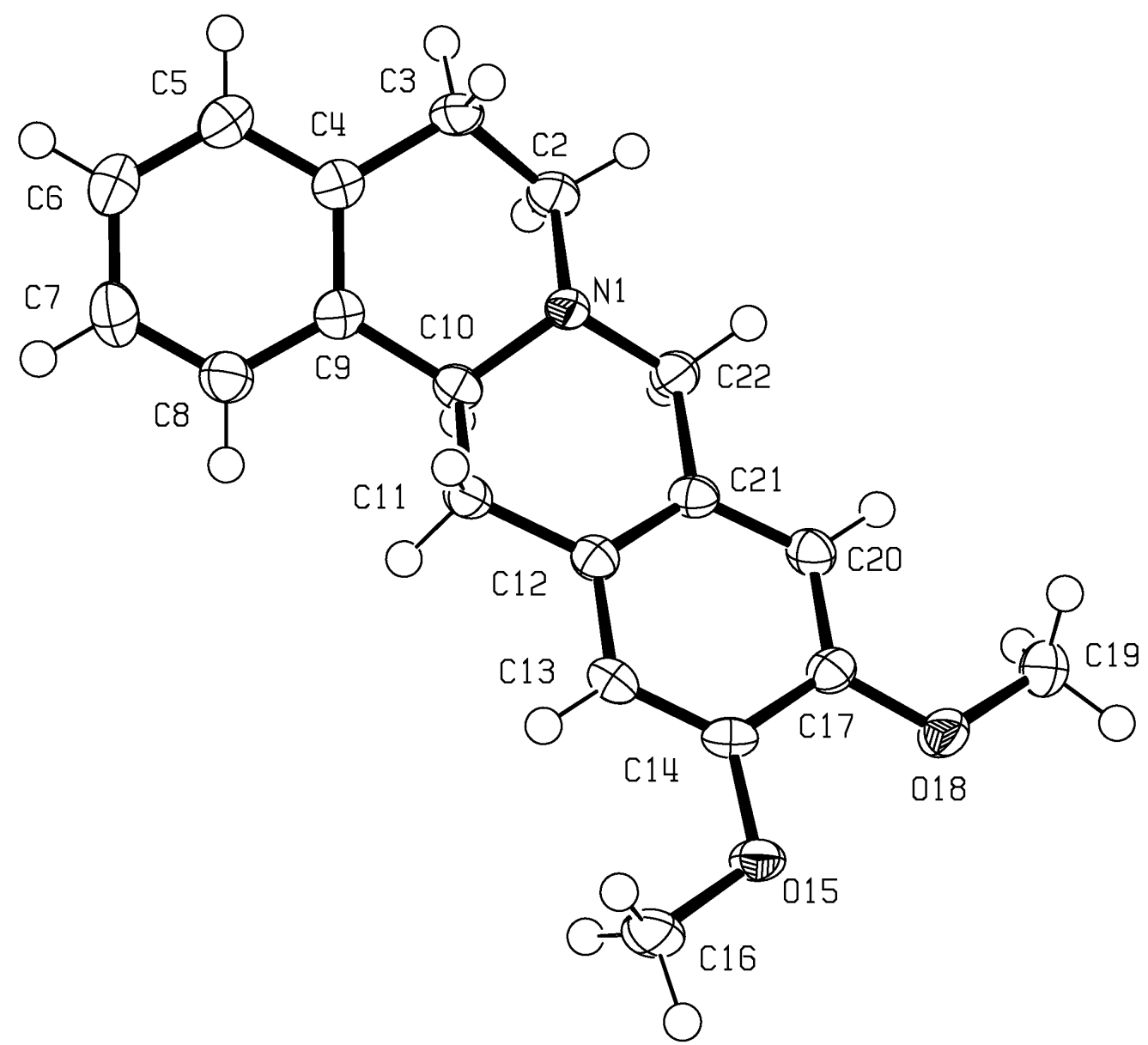

Figure S 152. ORTEP view of derivative (-)-4. Ellipsoid plots are drawn with $70 \%$ of probability. 


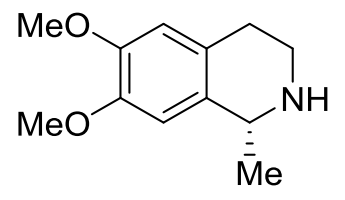

(+)-salsolidine

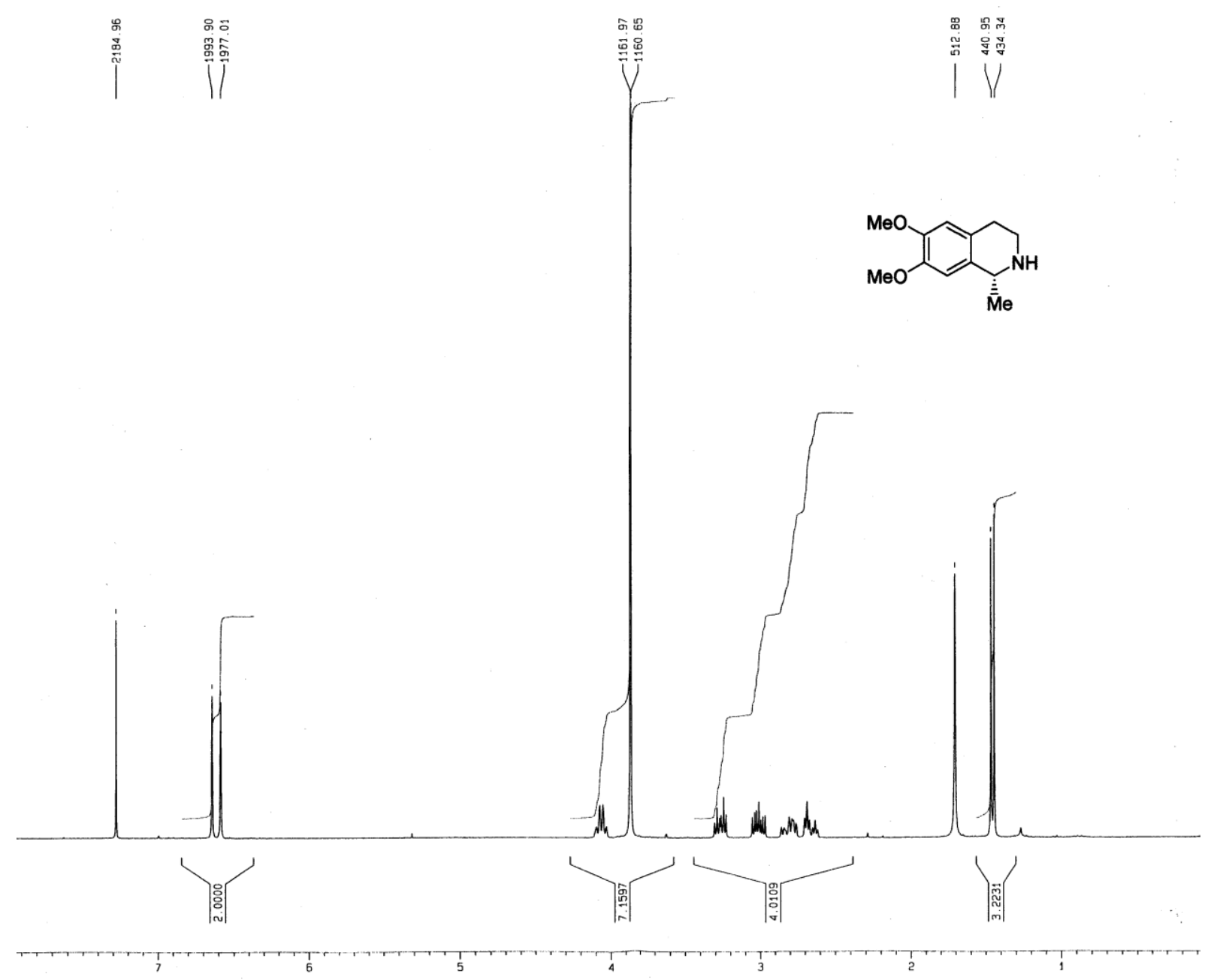

Figure S 153. ${ }^{1} \mathrm{H}$ NMR spectrum $\left(\mathrm{CDCl}_{3}, 300 \mathrm{MHz}\right)$ of $(+)$-salsolidine. 


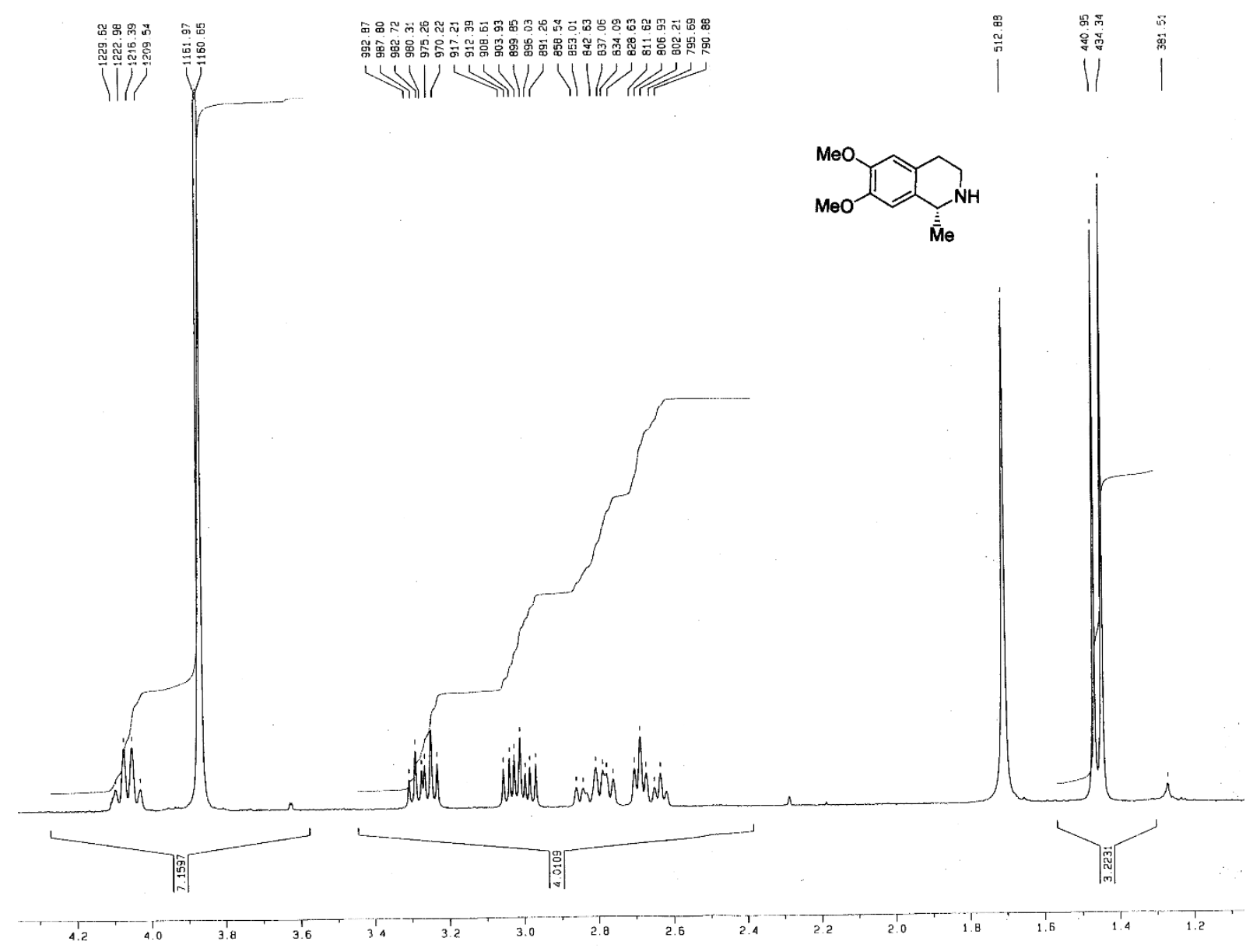

Figure S 154. ${ }^{1} \mathrm{H}$ NMR spectrum $\left(\mathrm{CDCl}_{3}, 300 \mathrm{MHz}\right)$ of $(+)$-salsolidine. 


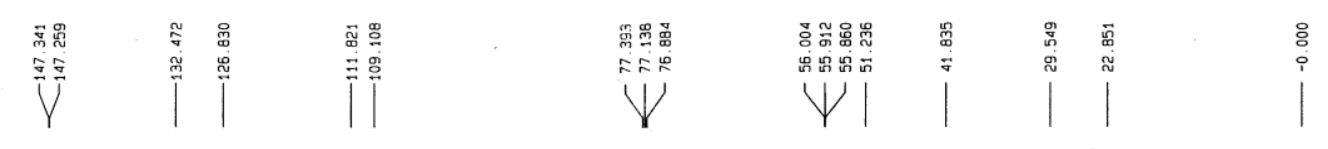
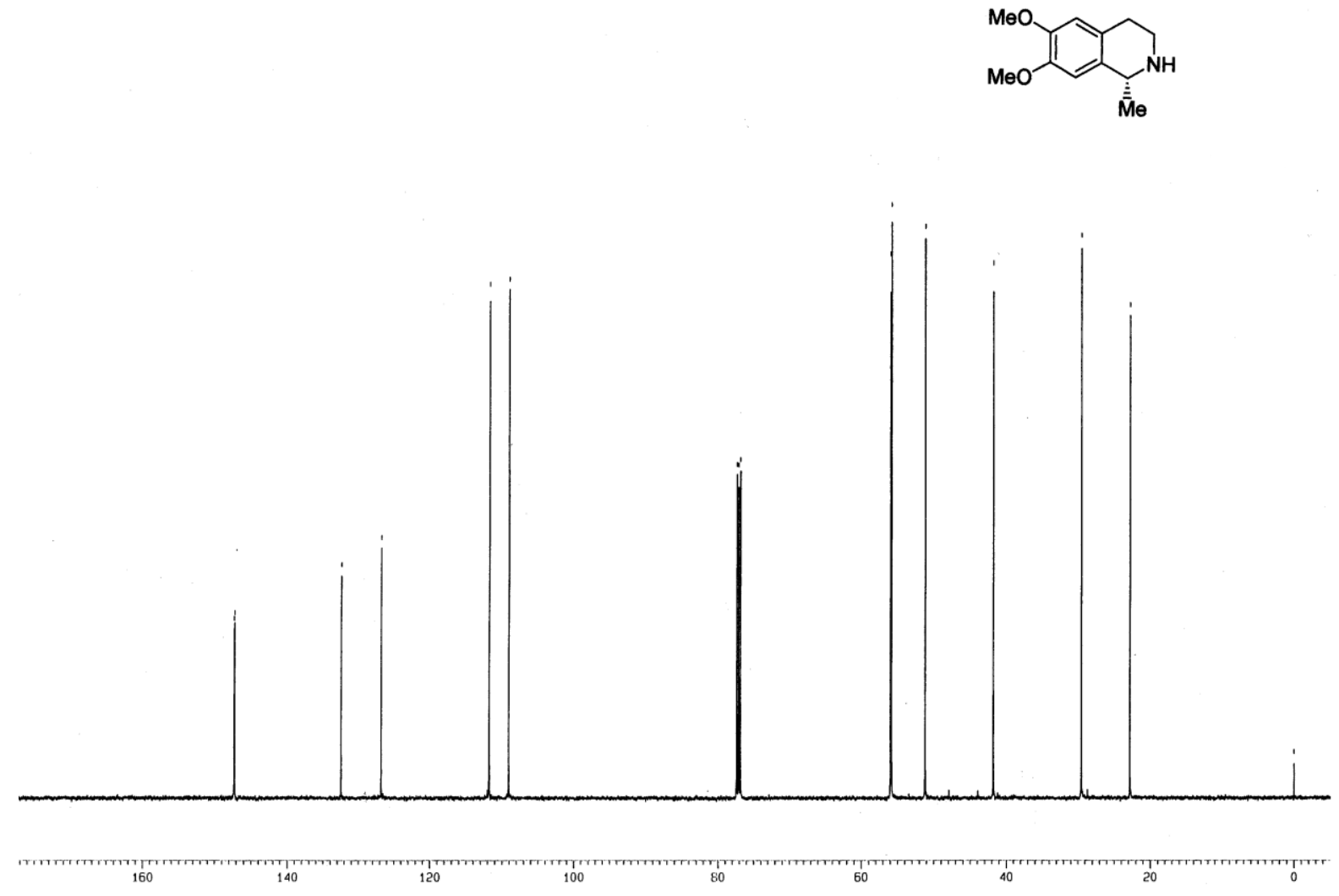

Figure S $155 .{ }^{13} \mathrm{C} \mathrm{NMR}$ spectrum $\left(\mathrm{CDCl}_{3}, 75 \mathrm{MHz}\right)$ of $(+)$-salsolidine. 


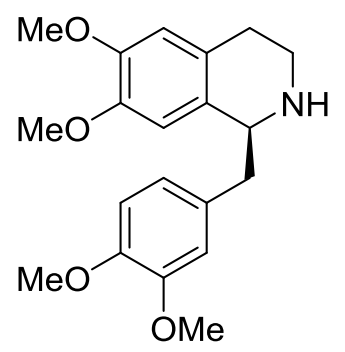

(S)-(-)-nor-laudanosine

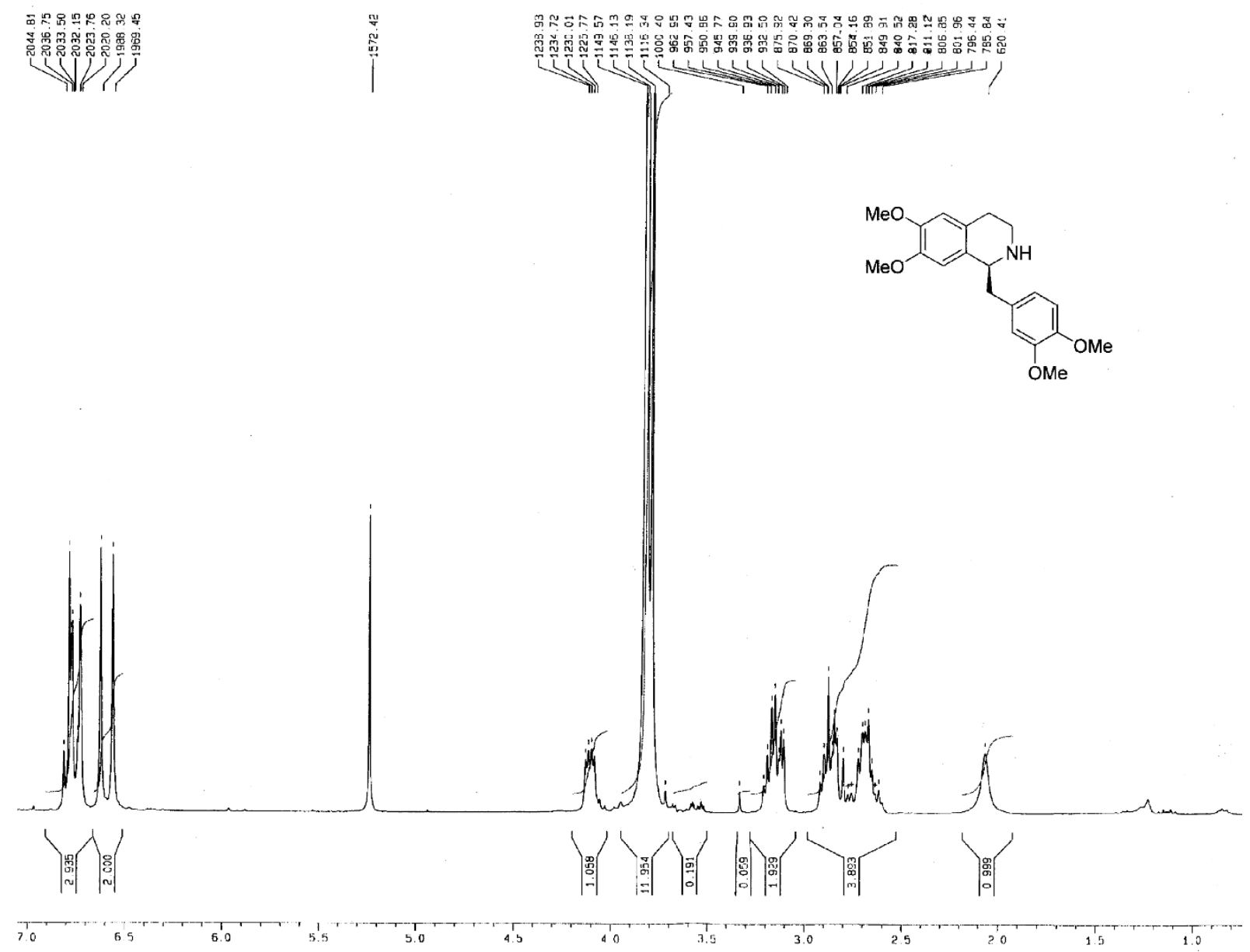

Figure S 156. ${ }^{1} \mathrm{H} \mathrm{NMR}\left(\mathrm{CDCl}_{3}, 300 \mathrm{MHz}\right)$ spectrum of (-)-norlaudanosine. 


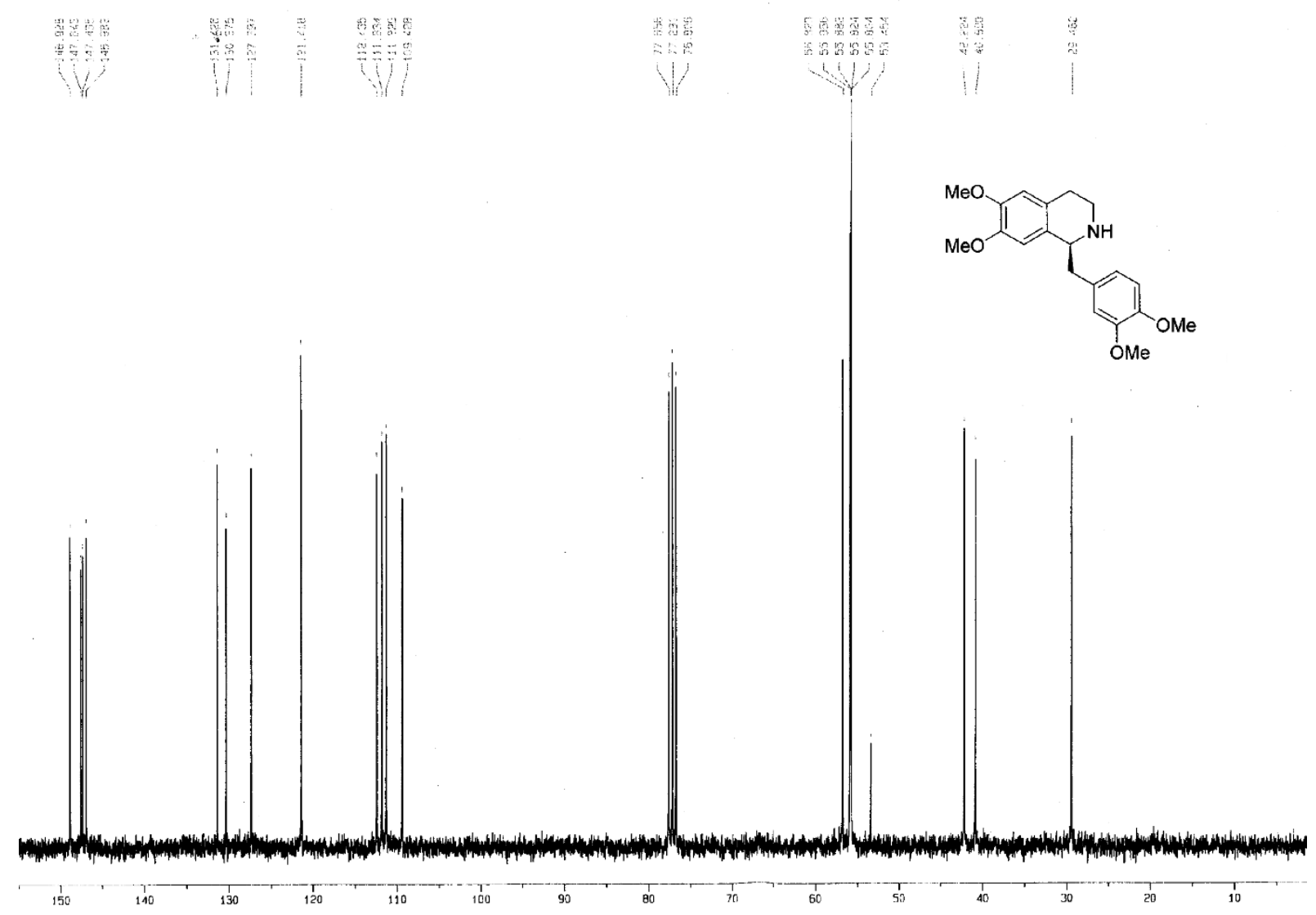

Figure S 157. $\left.{ }^{13} \mathrm{C} \mathrm{NMR} \mathrm{(CDCl} 3,75 \mathrm{MHz}\right)$ spectrum of (-)-norlaudanosine. 
<smiles>COc1cc2c(cc1OC)[C@H](C)NCC2</smiles><smiles>O=C(O)C(C(=O)O)C(C(=O)O)C(=O)Oc1ccccc1</smiles>

(+)-salsolidine•(-)-O,O'-Dibenzoyl-L-tartaric acid, (-)-12.
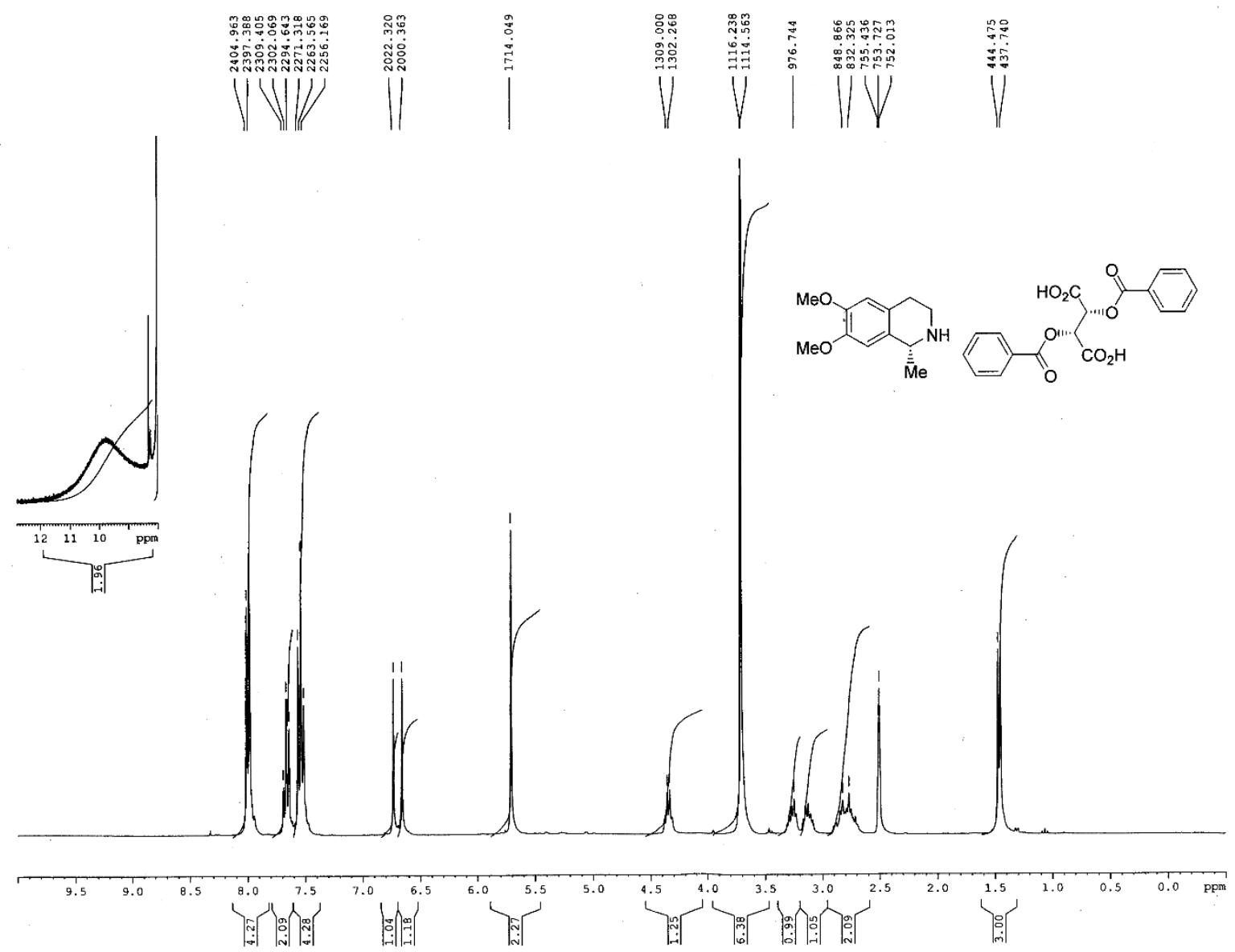

Figure S 158. ${ }^{1} \mathrm{H}$ NMR spectrum (DMSO-d6, $300 \mathrm{MHz}$ ) of (+)-salsolidine•(-)-O,O'-Dibenzoyl-L-tartaric acid, (-)-12. 


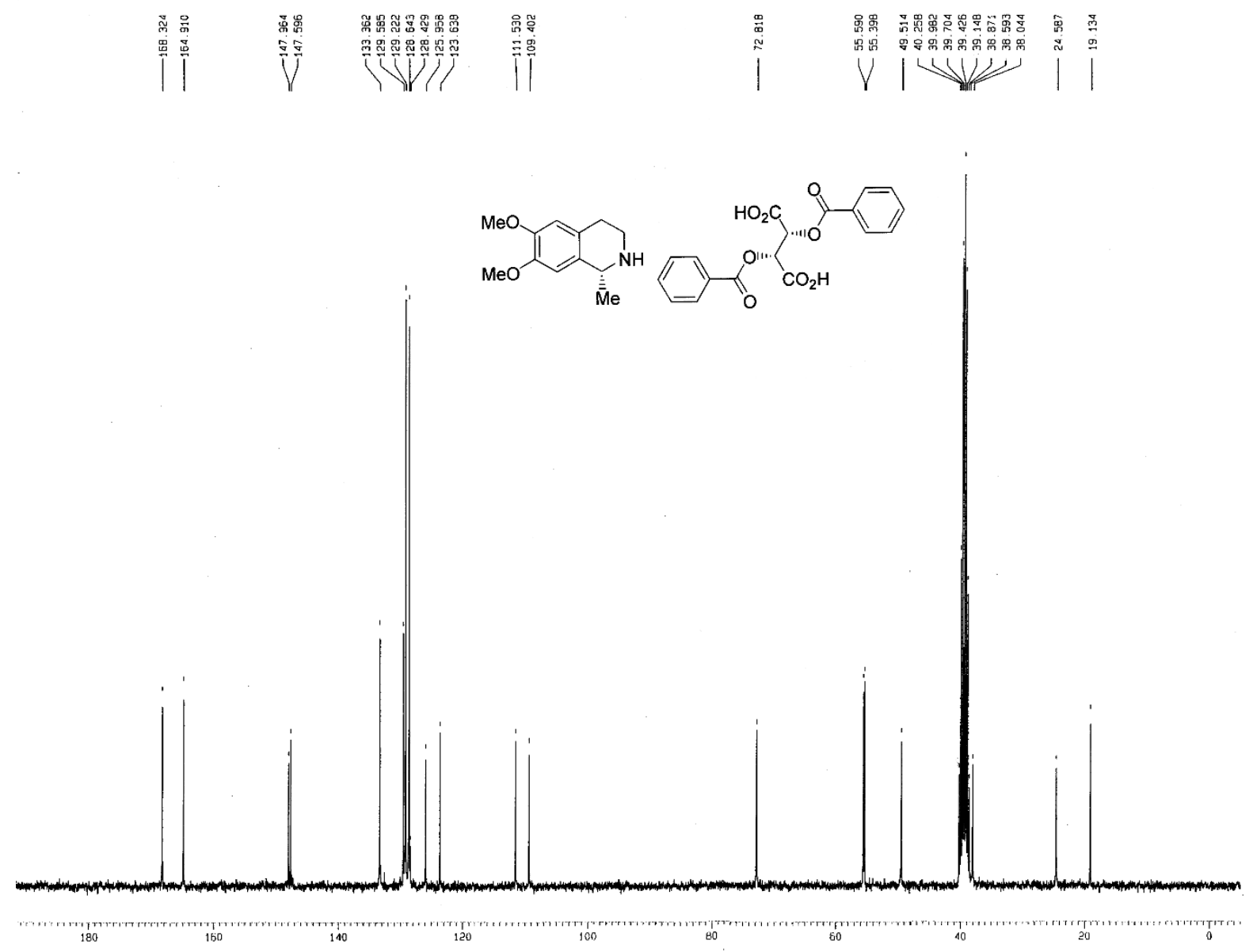

Figure S 159. ${ }^{13} \mathrm{C}$ NMR spectrum (DMSO- $\mathrm{d}_{6}, 75 \mathrm{MHz}$ ) of (+)-salsolidine•(-)-O,O'-Dibenzoyl-L-tartaric acid, (-)-12. 


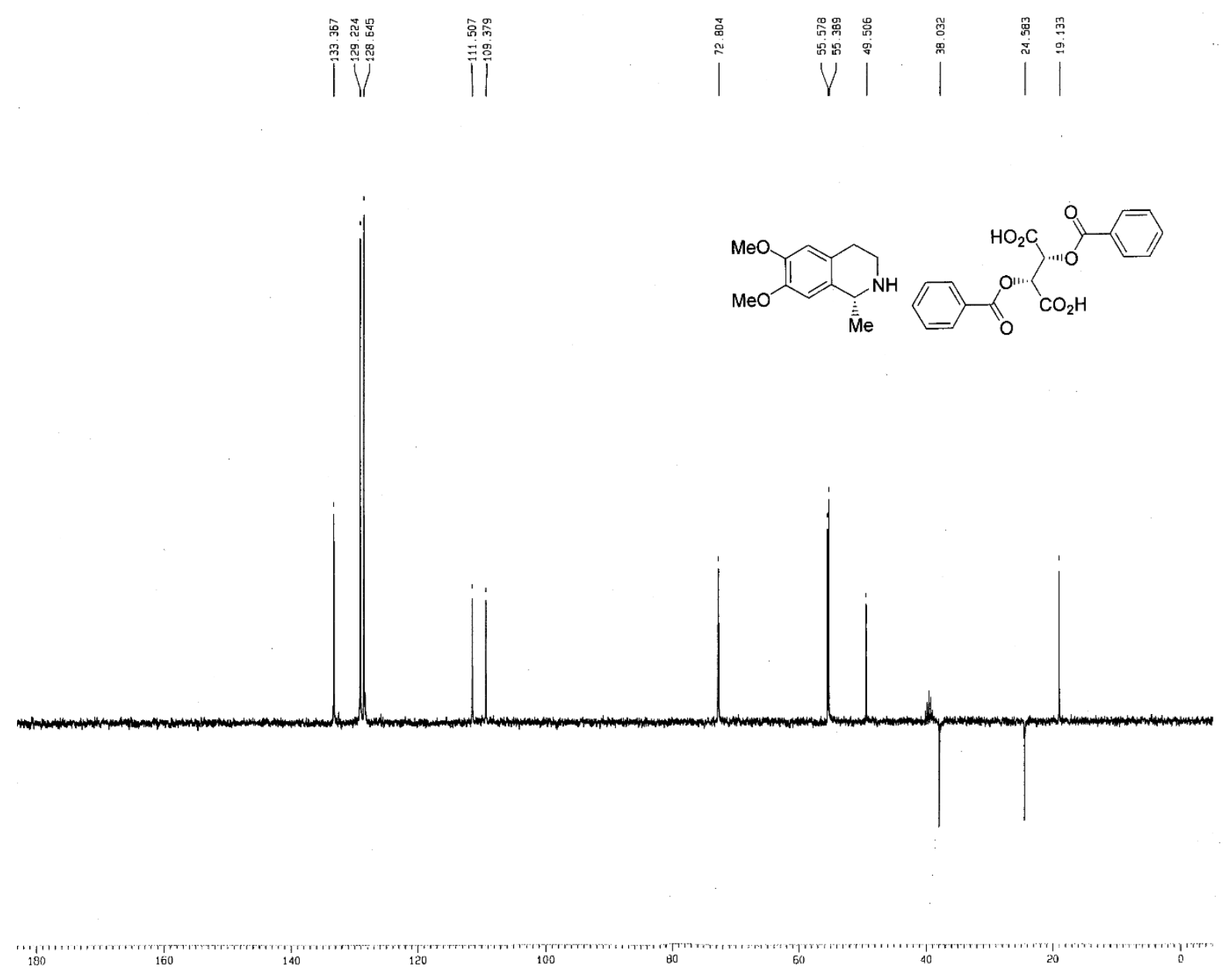

Figure S 160. DEPT C NMR spectrum (DMSO-d6, $75 \mathrm{MHz}$ ) of (+)-salsolidine•(-)-O,O'-Dibenzoyl-Ltartaric acid, (-)-12. 
<smiles>COc1ccc(C[C@@H]2NCCc3cc(OC)c(OC)cc32)cc1OC</smiles><smiles>CC(=O)N[C@@H](CC(C)C)C(=O)O</smiles>

(-)-norlaudanosine $\bullet(-)-N$-acetyl-L-leucine, $(-)-13$.
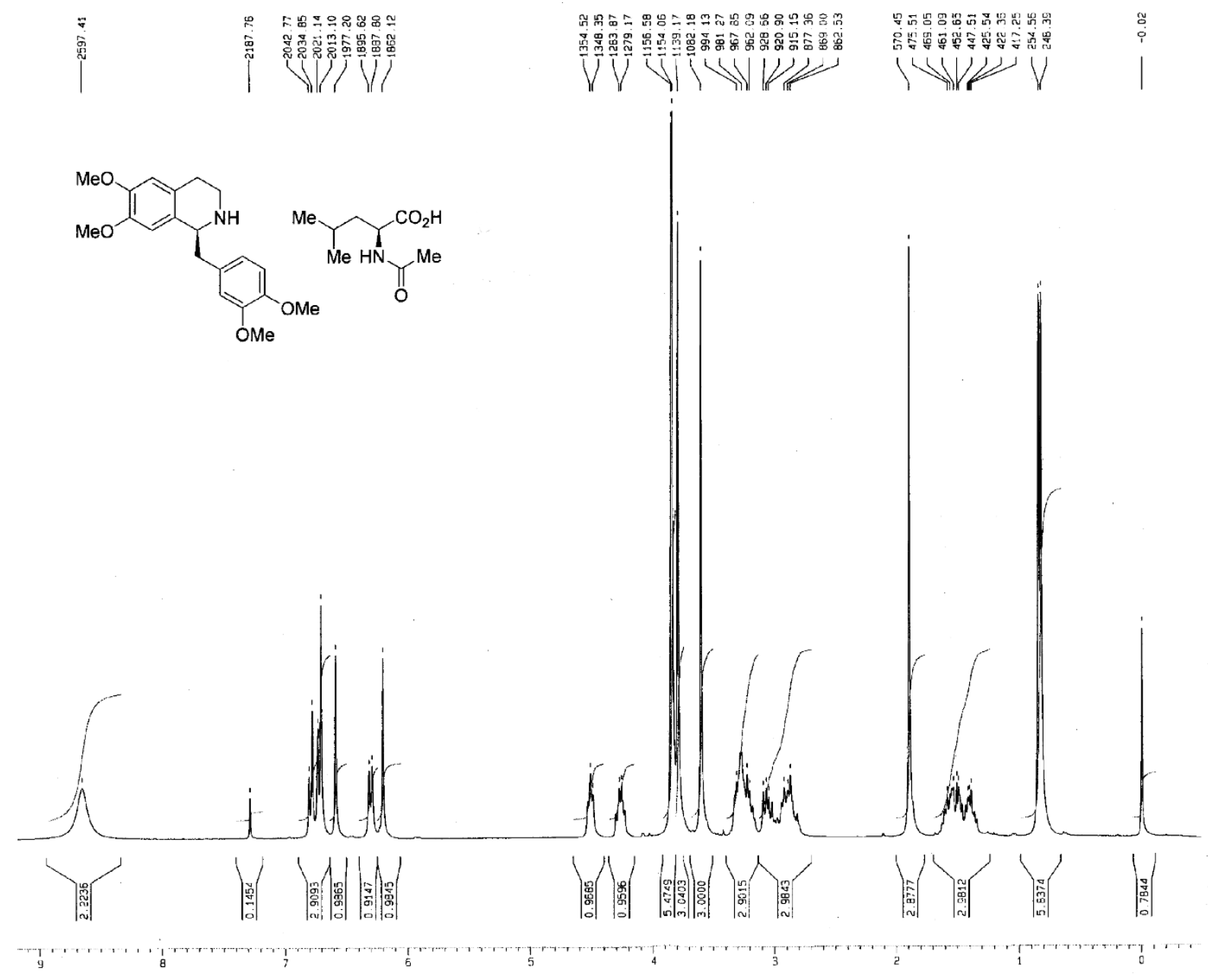

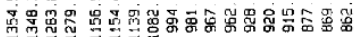

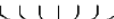

Figure S 161. ${ }^{1} \mathrm{H} \mathrm{NMR}\left(\mathrm{CDCl}_{3}, 300 \mathrm{MHz}\right)$ spectrum of (-)-norlaudanosine $(-)-\mathrm{N}$-acetyl-L-leucine, (-)13. 


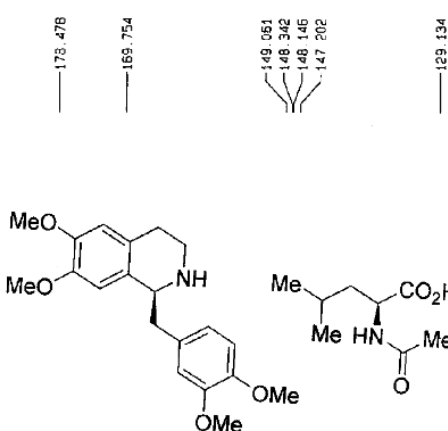

||

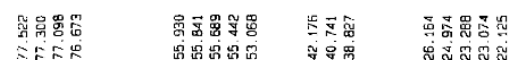

W) II UV

W WI HI

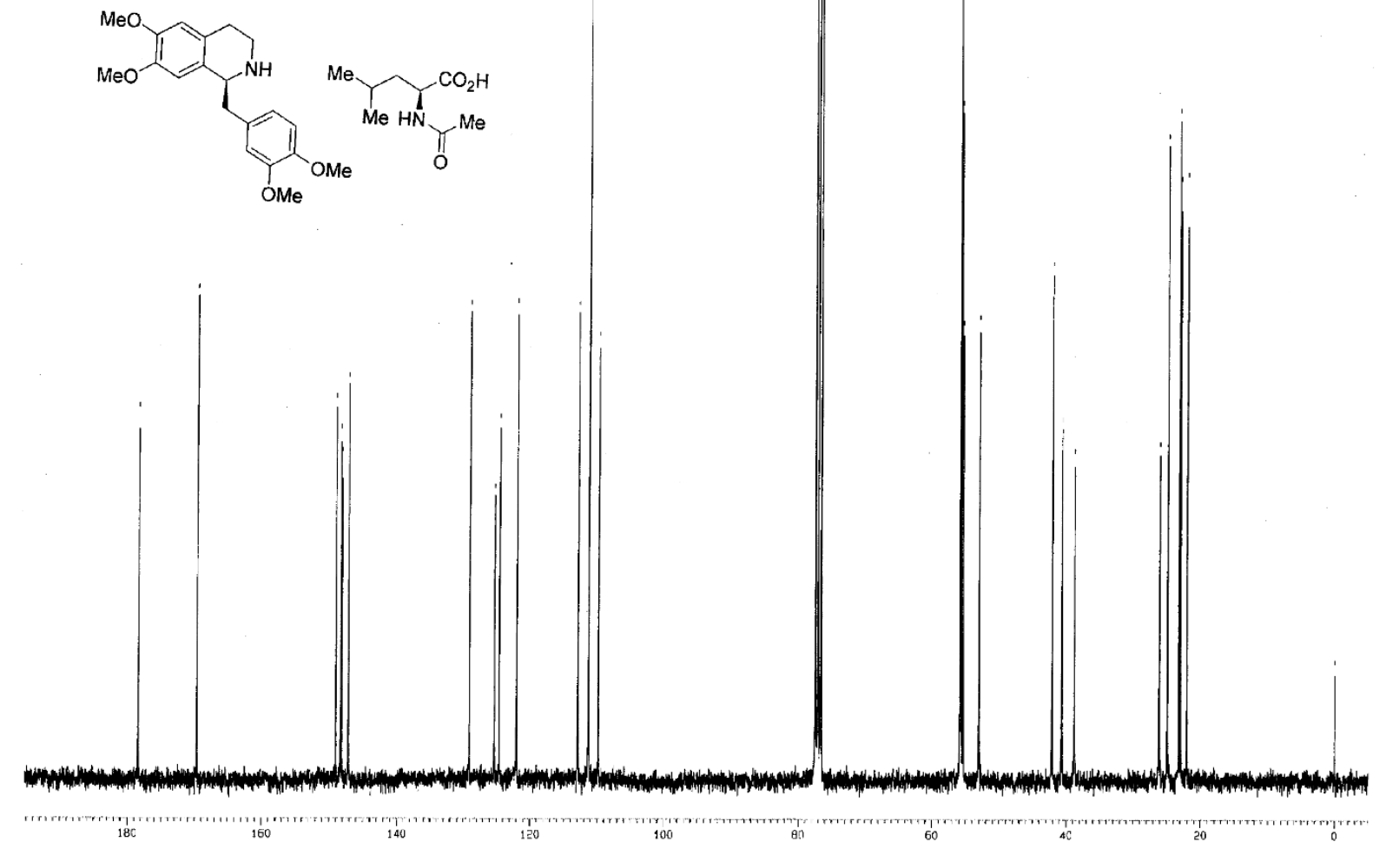

Figure S 162. ${ }^{13} \mathrm{C} \mathrm{NMR}\left(\mathrm{CDCl}_{3}, 75 \mathrm{MHz}\right)$ spectrum of (-)-norlaudanosine $(-)-N$-acetyl-L-leucine, (-)-13. 


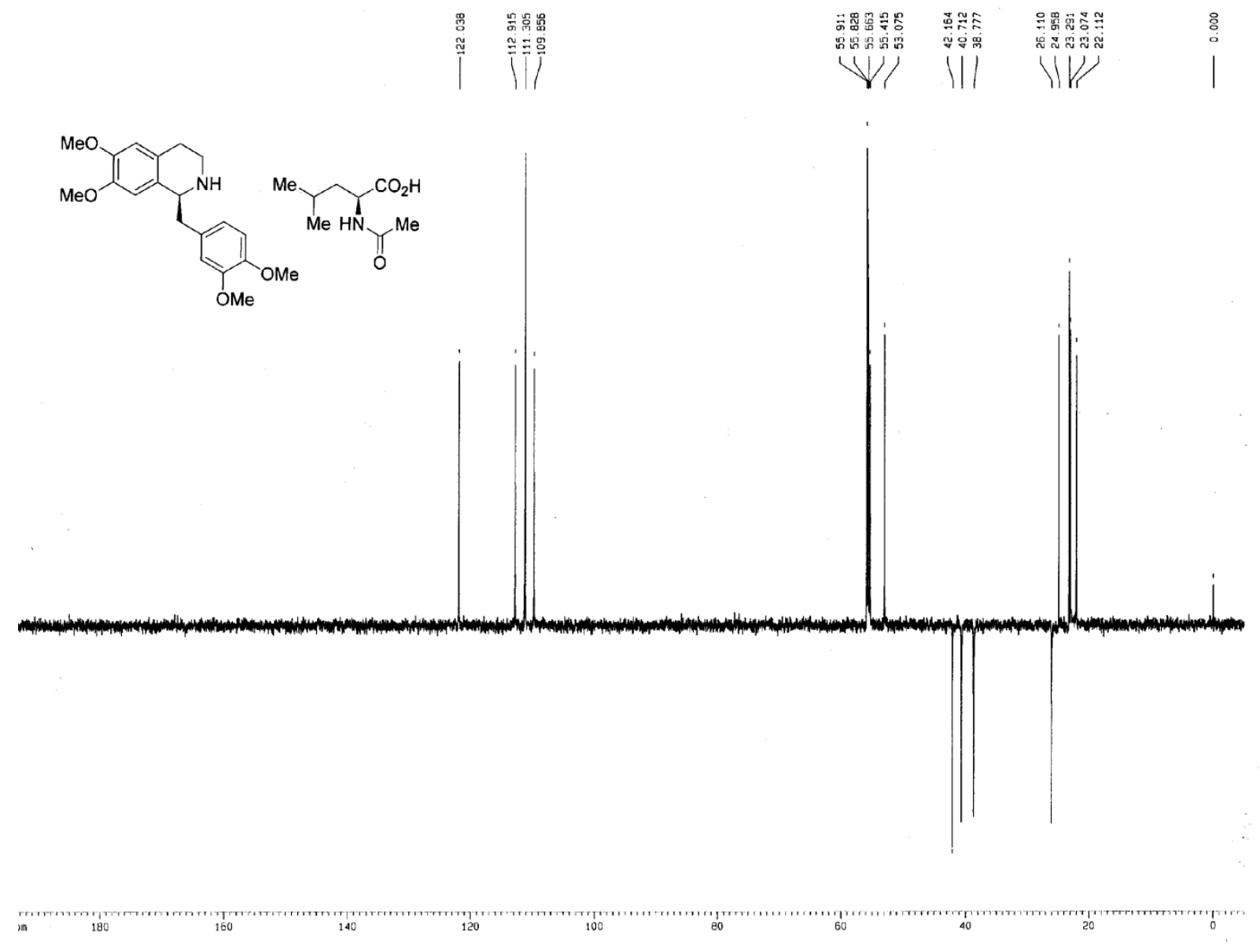

Figure S 163. DEPT C NMR spectrum (DMSO-d 6 , $75 \mathrm{MHz}$ ) of $(-)$-norlaudanosine $\bullet(-)-N$-acetyl-Lleucine, (-)-13. 
<smiles>COc1cc2c(cc1OC)[C@H](C)NCC2</smiles><smiles>CC(C)(C)P(=O)(S)c1ccccc1</smiles>

$(R)-(+)$-salsolidine $(85: 15$ er $) \bullet(+)-14$

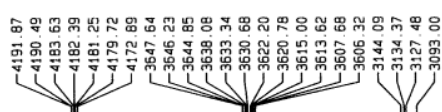

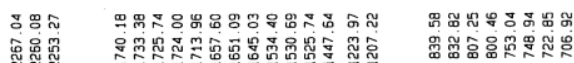

诲总总
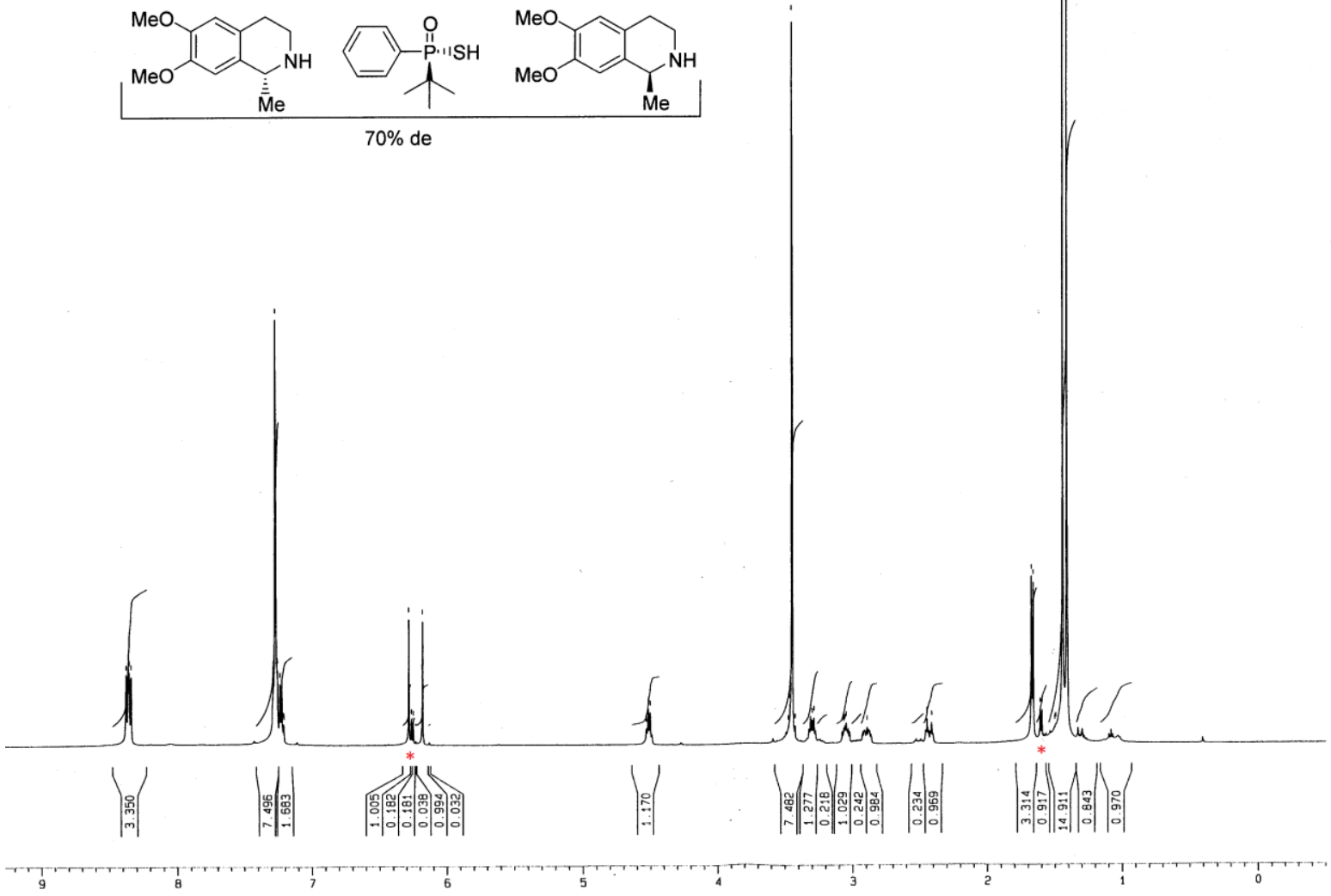

Figure S 164. ${ }^{1} \mathrm{H}$ NMR $\left(\mathrm{C}_{6} \mathrm{D}_{6}, 500 \mathrm{MHz}\right)$ spectrum of $(+)$-salsolidine $(85: 15$ er $)$ with (+)-tertbutylphenylphosphinothioic acid. $(\gamma=1.55)$. Splitting signals are indicated with an asterisk $\left(^{*}\right)$. 


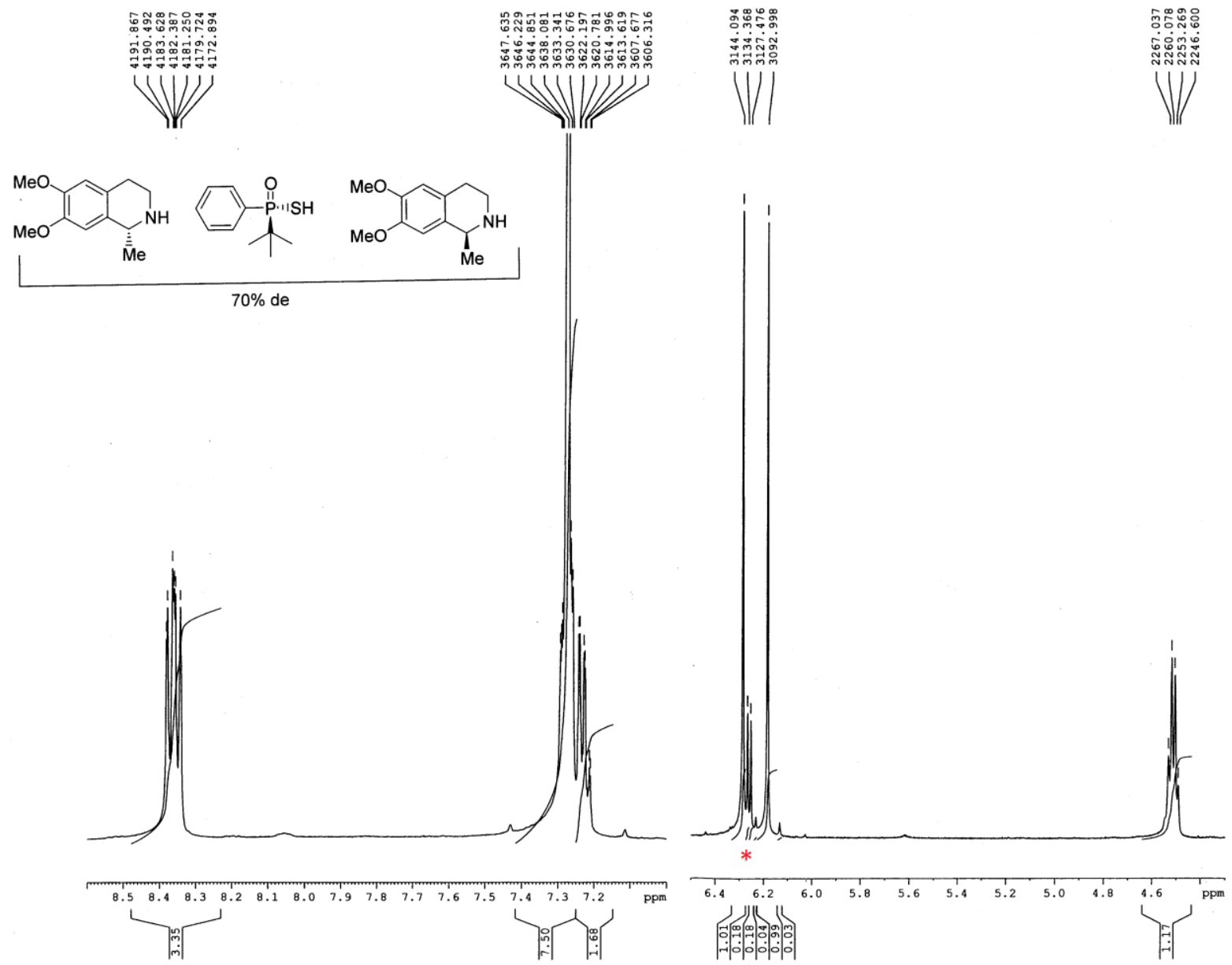

Figure S 165. ${ }^{1} \mathrm{H}$ NMR $\left(\mathrm{C}_{6} \mathrm{D}_{6}, 500 \mathrm{MHz}\right)$ spectrum of $(+)$-salsolidine $(85: 15 \mathrm{er})$ with $(+)$-tertbutylphenylphosphinothioic acid. $(\gamma=1.55)$. Splitting signals are indicated with an asterisk $\left({ }^{*}\right)$. 


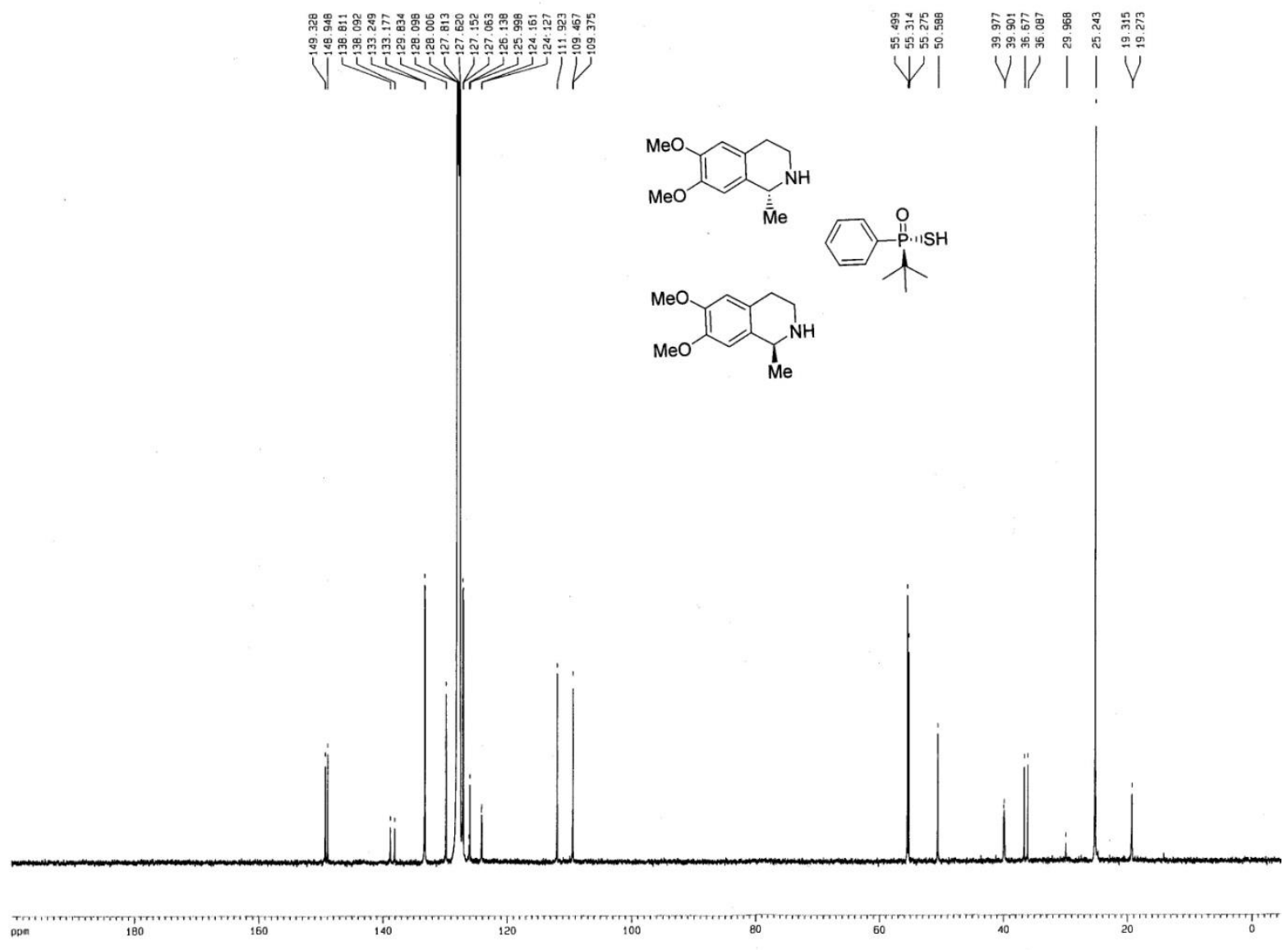

Figure S 166. ${ }^{13} \mathrm{C}$ NMR $\left(\mathrm{C}_{6} \mathrm{D}_{6}, 125 \mathrm{MHz}\right)$ spectrum of (+)-salsolidine $(85: 15 \mathrm{er})$ with (+)-tertbutylphenylphosphinothioic acid. $(\gamma=1.55)$. 


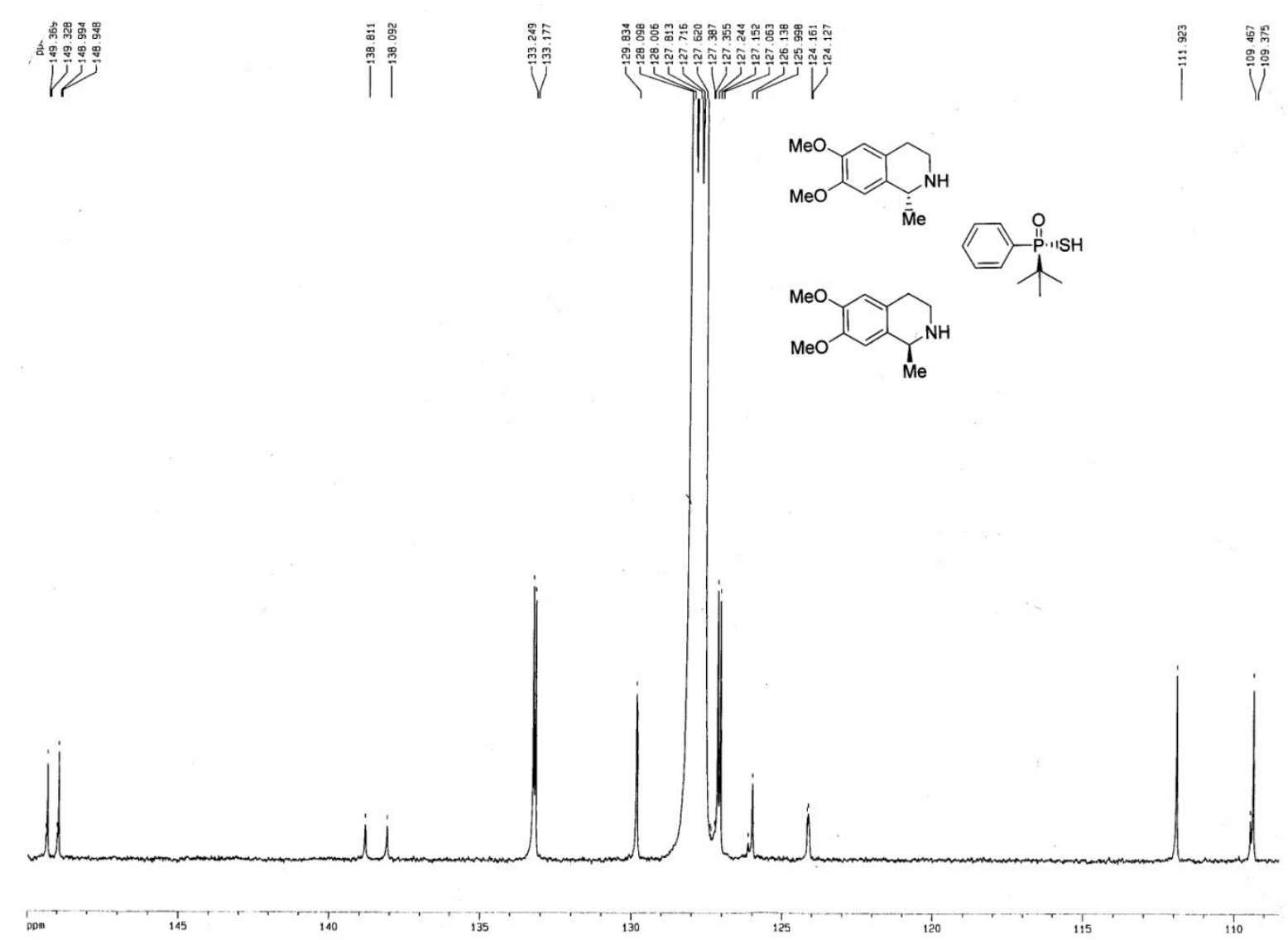

Figure S 167. ${ }^{13} \mathrm{C}$ NMR $\left(\mathrm{C}_{6} \mathrm{D}_{6}, 125 \mathrm{MHz}\right)$ spectrum of (+)-salsolidine (85:15 er) with (+)-tertbutylphenylphosphinothioic acid. $(\gamma=1.55)$. 


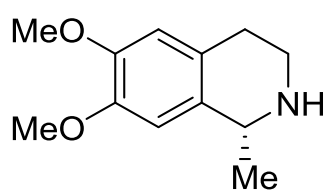<smiles>CC(C)(C)P(=O)(S)c1ccccc1</smiles>

(+)-salsolidine (99:1 er) $\bullet(+)-14$

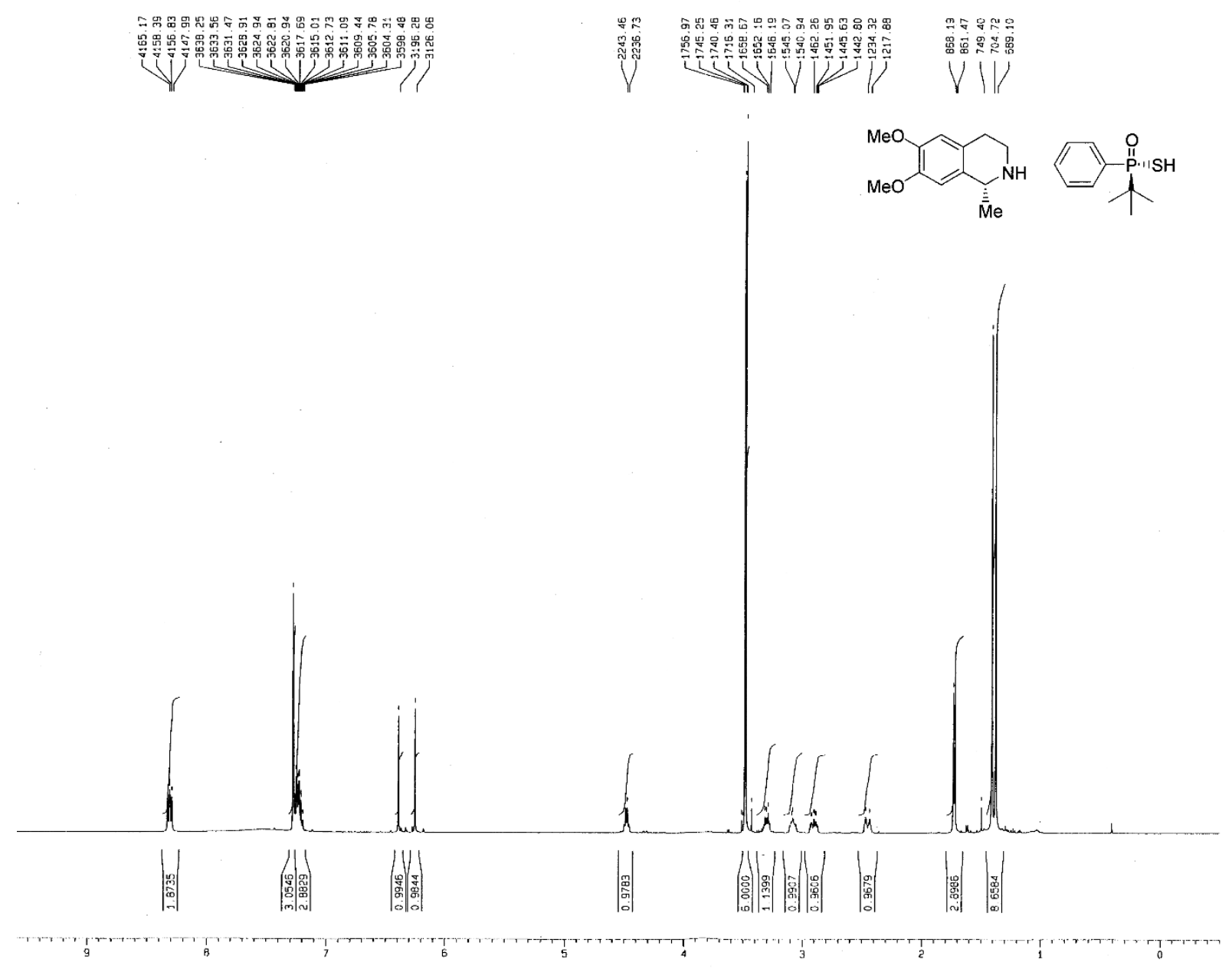

Figure S 168. ${ }^{1} \mathrm{H}$ NMR $\left(\mathrm{C}_{6} \mathrm{D}_{6}, 500 \mathrm{MHz}\right)$ spectrum of $(+)$-salsolidine $(99: 1$ er) with $(+)$-tertbutylphenylphosphinothioic acid. $(\gamma=1.0)$. 


\section{S171}

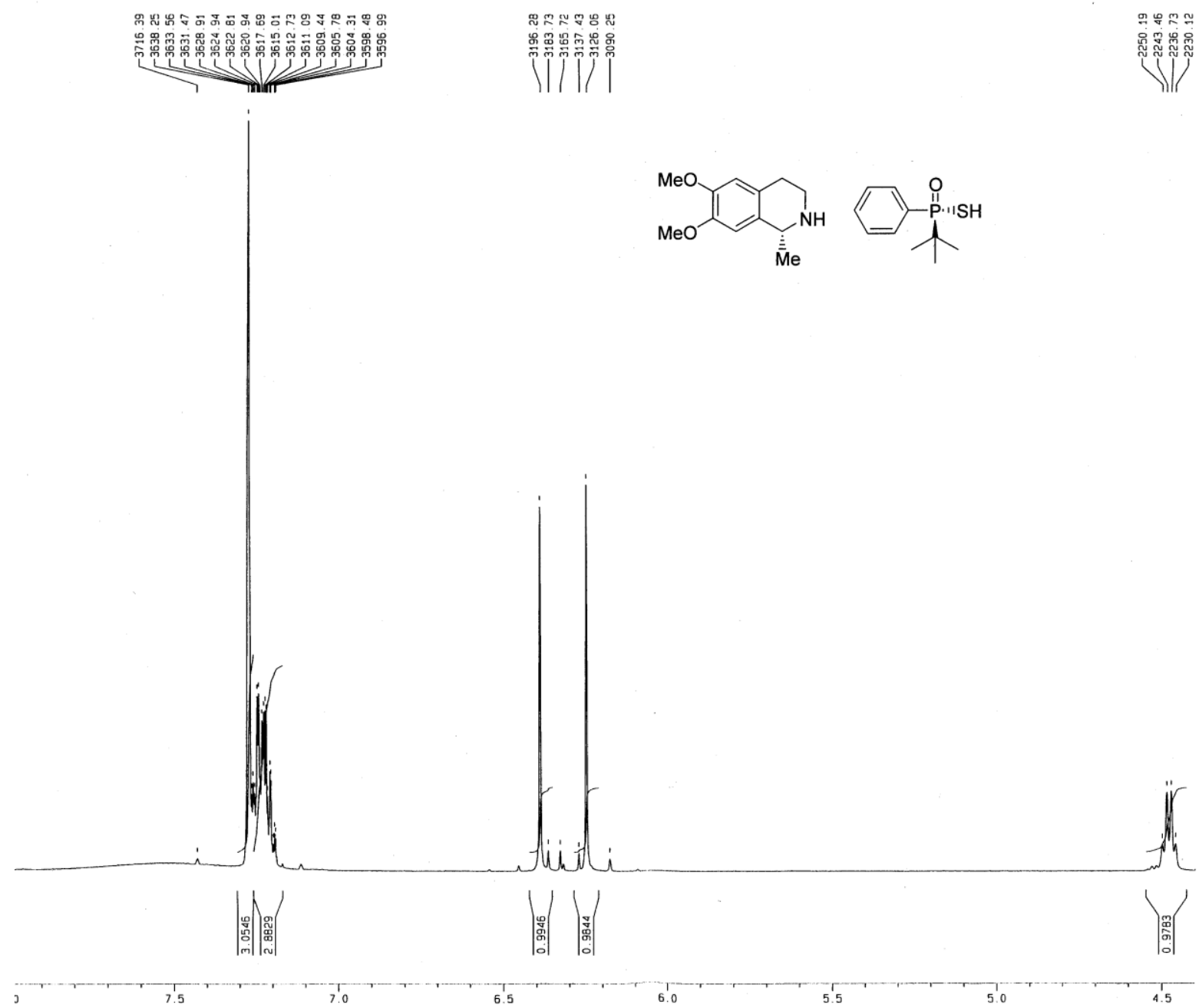

Figure S 169. ${ }^{1} \mathrm{H} \quad \mathrm{NMR}\left(\mathrm{C}_{6} \mathrm{D}_{6}, 500 \mathrm{MHz}\right)$ spectrum of (+)-salsolidine (99:1) with (+)-tertbutylphenylphosphinothioic acid. $(\gamma=1.0)$. 


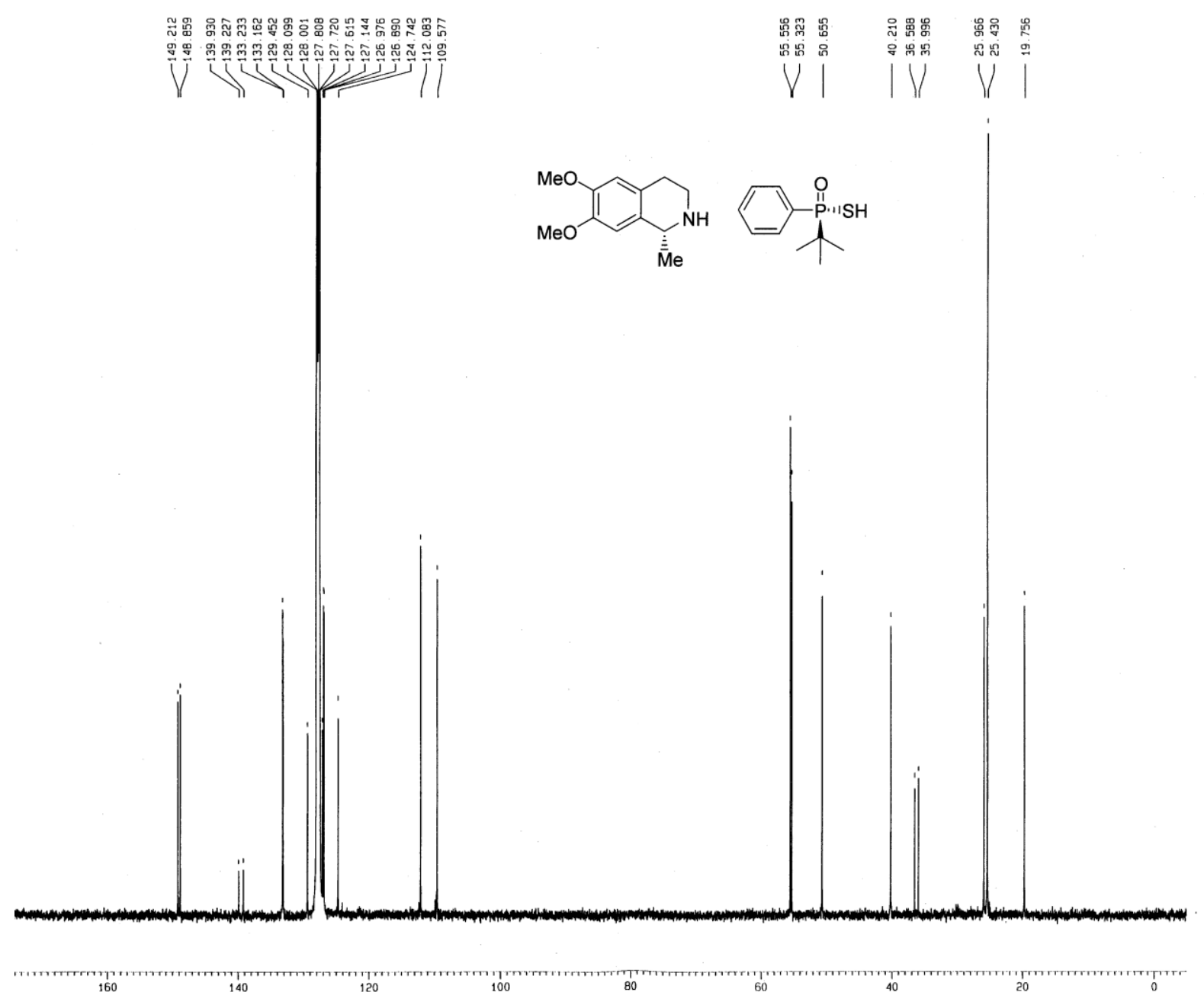

Figure S 170. ${ }^{13} \mathrm{C}$ NMR $\left(\mathrm{C}_{6} \mathrm{D}_{6}, 125 \mathrm{MHz}\right.$, transients = 1800) spectrum of $(+)$-salsolidine (>98:2 er) with (+)-tert-butylphenylphosphinothioic acid. $(\gamma=1.0)$. 
<smiles>COc1ccc(C[C@@H]2NCCc3cc(OC)c(OC)cc32)cc1OC</smiles>

(-)-norlaudanosine $\bullet(+)-14,90: 10 \mathrm{dr}$.

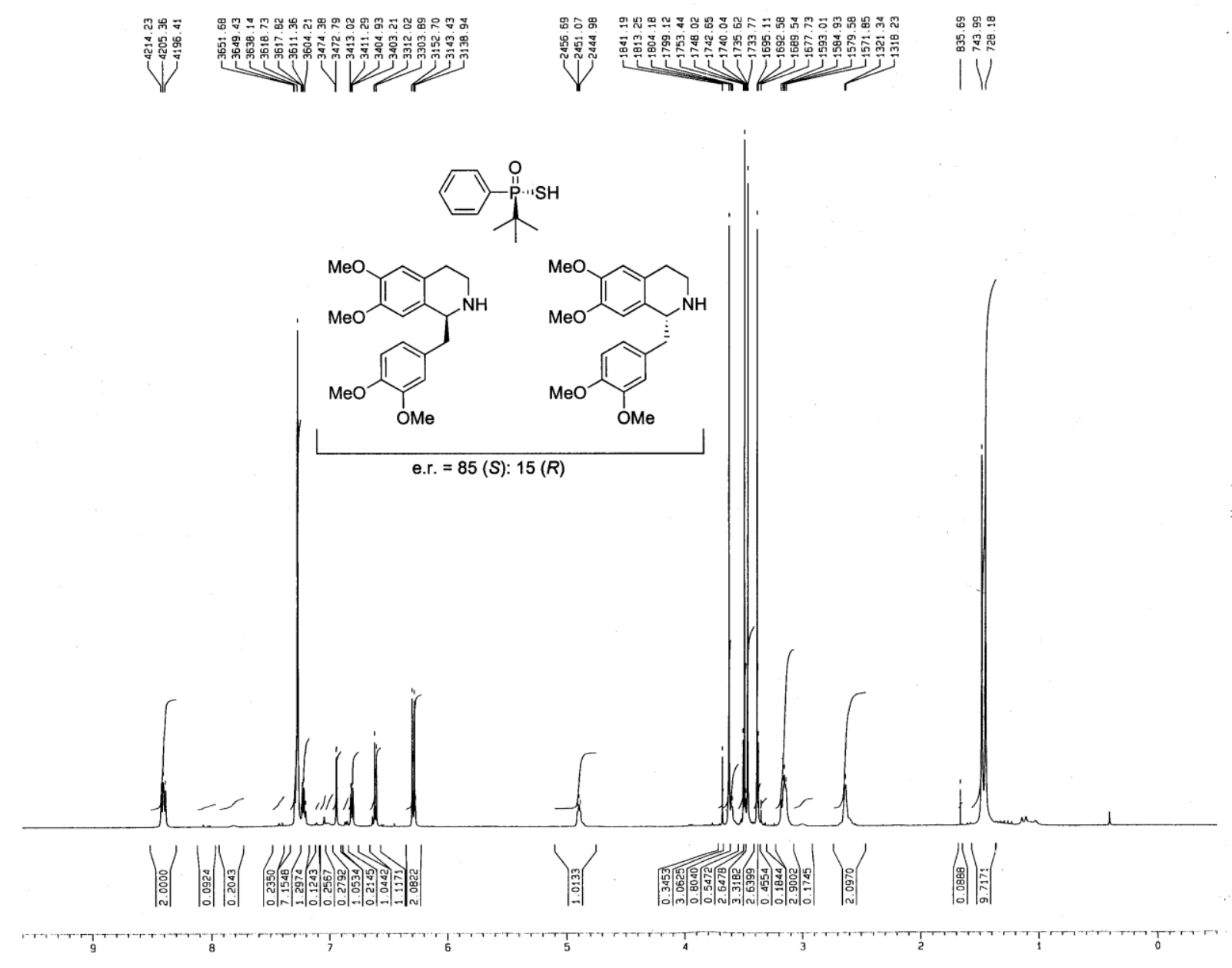

Figure $\mathrm{S}$ 171. ${ }^{1} \mathrm{H}$ NMR $\left(\mathrm{C}_{6} \mathrm{D}_{6}, 500 \mathrm{MHz}\right)$ spectrum of (-)-nor-laudanosine $(90: 10 \mathrm{er})$ with $(+)$-tertbutylphenylphosphinothioic acid. $(\gamma=1.07)$. 

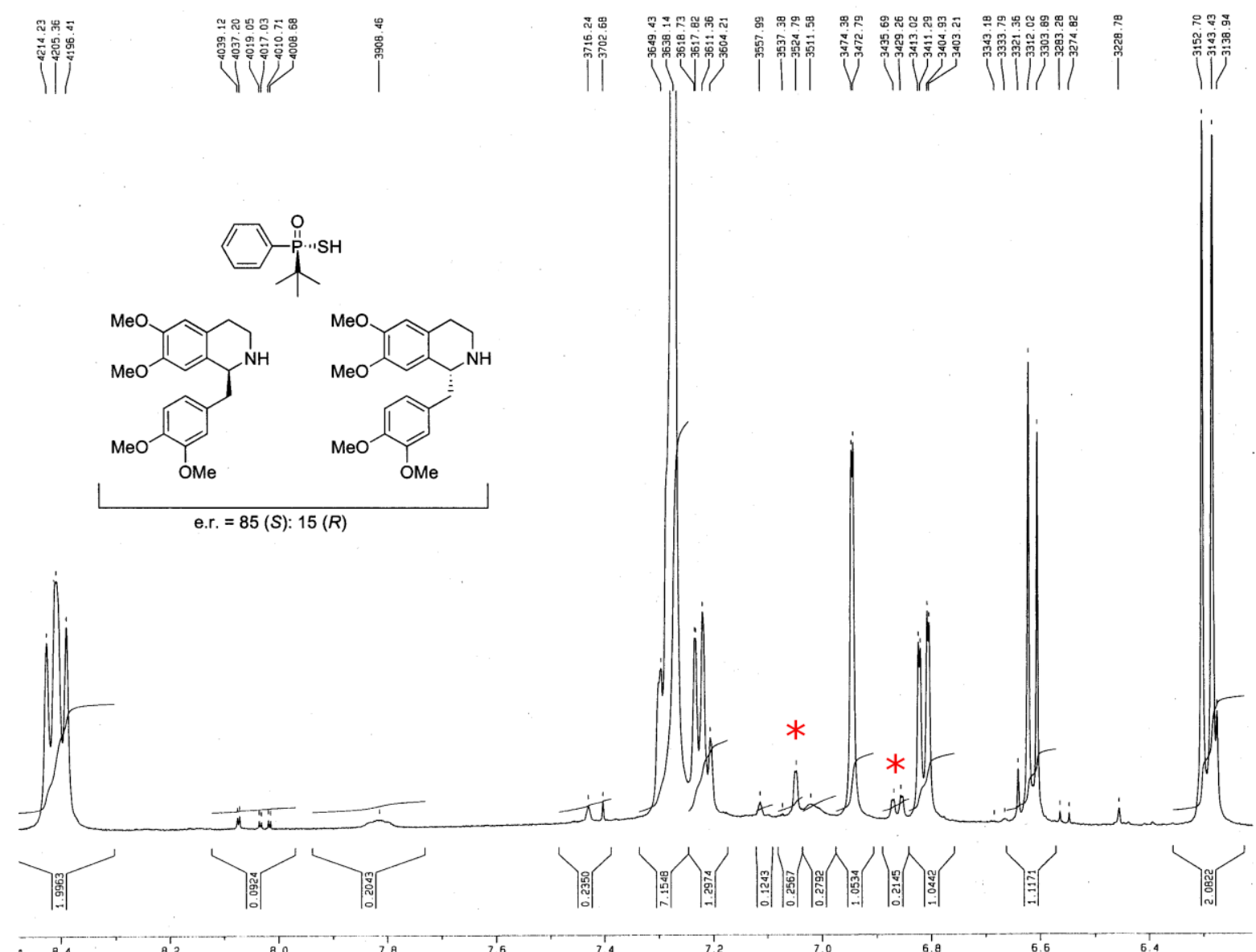

Figure S 172. ${ }^{1} \mathrm{H}$ NMR $\left(\mathrm{C}_{6} \mathrm{D}_{6}, 500 \mathrm{MHz}\right)$ spectrum of (-)-nor-laudanosine (90:10 er) with (+)-tertbutylphenylphosphinothioic acid. $(\gamma=1.07)$. Splitting signals are indicated with an asterisk $\left(^{*}\right)$ 

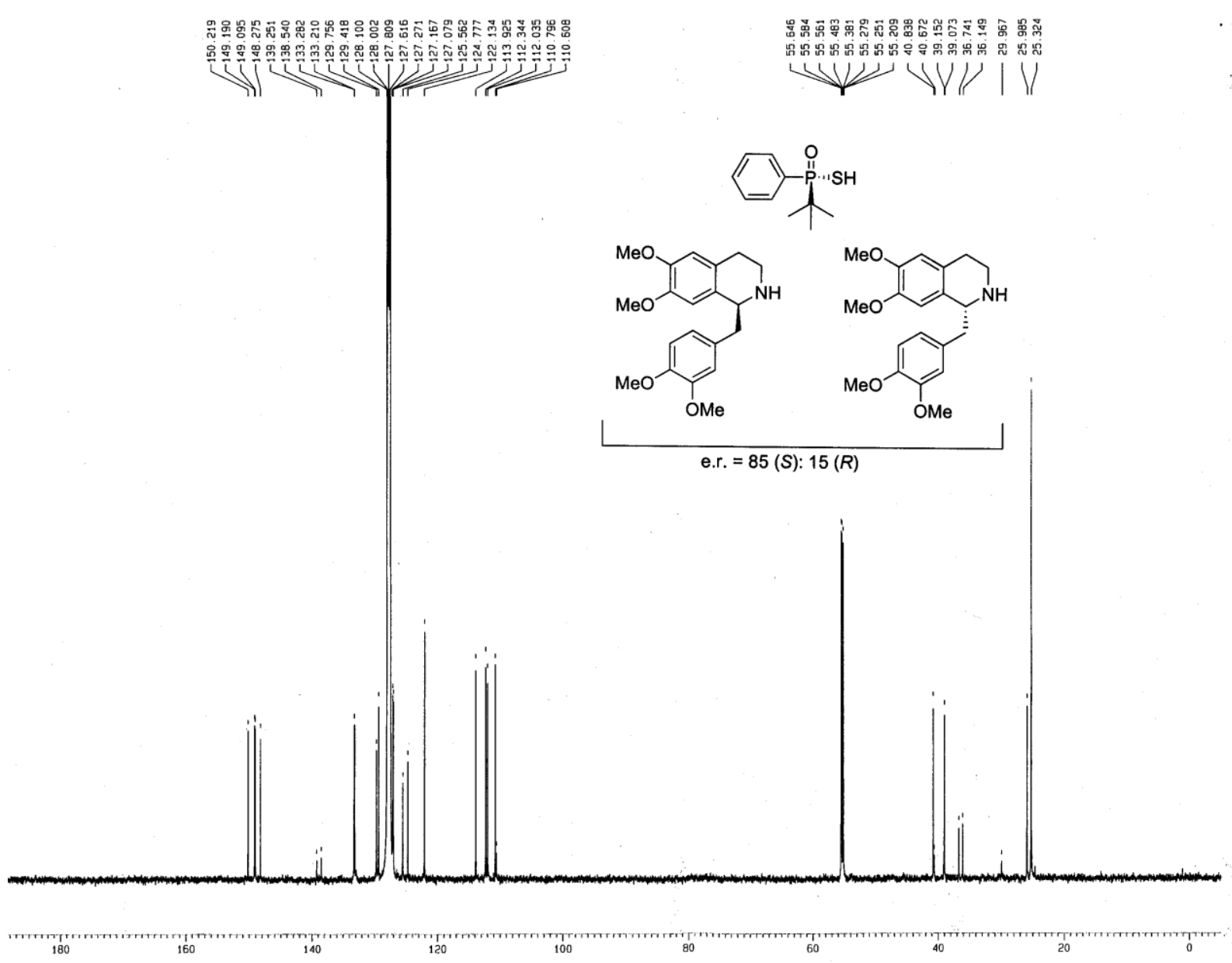

Figure S 173. ${ }^{13} \mathrm{C}$ NMR $\left(\mathrm{C}_{6} \mathrm{D}_{6}, 125 \mathrm{MHz}\right)$ spectrum of (-)-nor-laudanosine (90:10 er) with (+)-tertbutylphenylphosphinothioic acid. $(\gamma=1.07)$. 


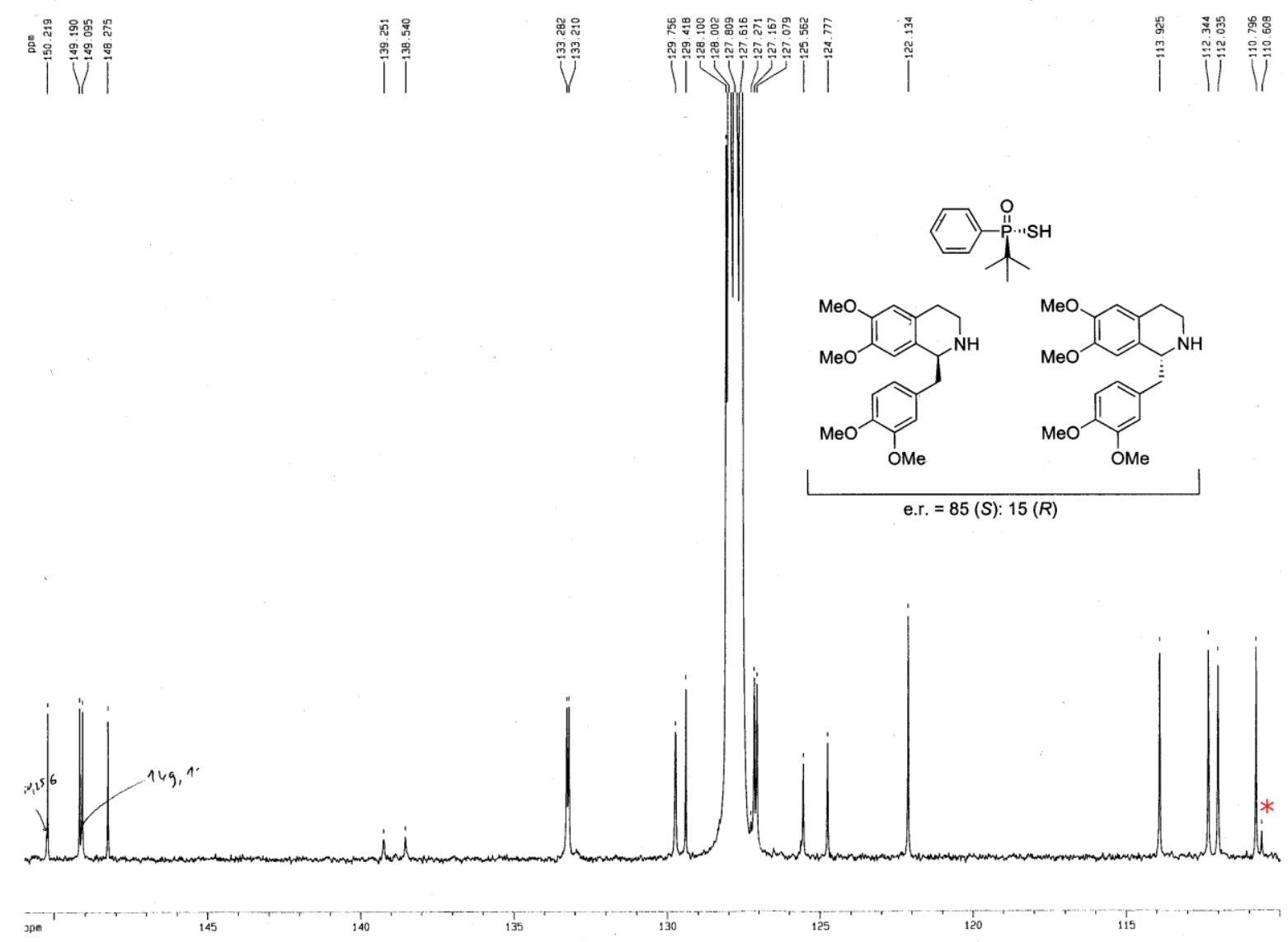

Figure S 174. ${ }^{13} \mathrm{C}$ NMR $\left(\mathrm{C}_{6} \mathrm{D}_{6}, 125 \mathrm{MHz}\right)$ spectrum of (-)-nor-laudanosine (90:10 er) with (+)-tertbutylphenylphosphinothioic acid. $(\gamma=1.07)$. Splitting signals are indicated with an asterisk $\left({ }^{*}\right)$. 

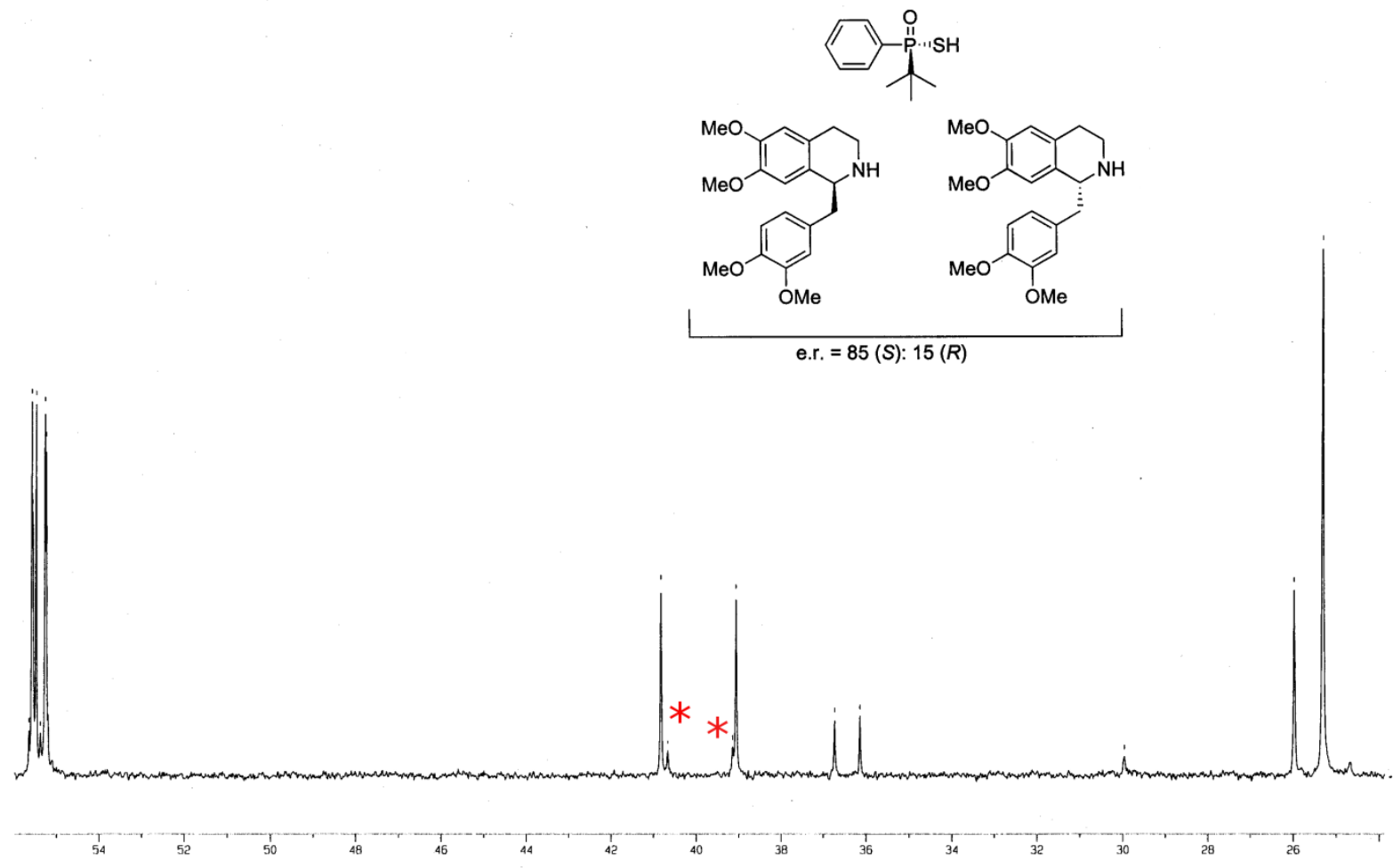

Figure S 175. ${ }^{13} \mathrm{C}$ NMR $\left(\mathrm{C}_{6} \mathrm{D}_{6}, 125 \mathrm{MHz}\right)$ spectrum of (-)-nor-laudanosine (90:10 er) with (+)-tertbutylphenylphosphinothioic acid. $(\gamma=1.07)$. Splitting signals are indicated with an asterisk $\left(^{*}\right)$. 


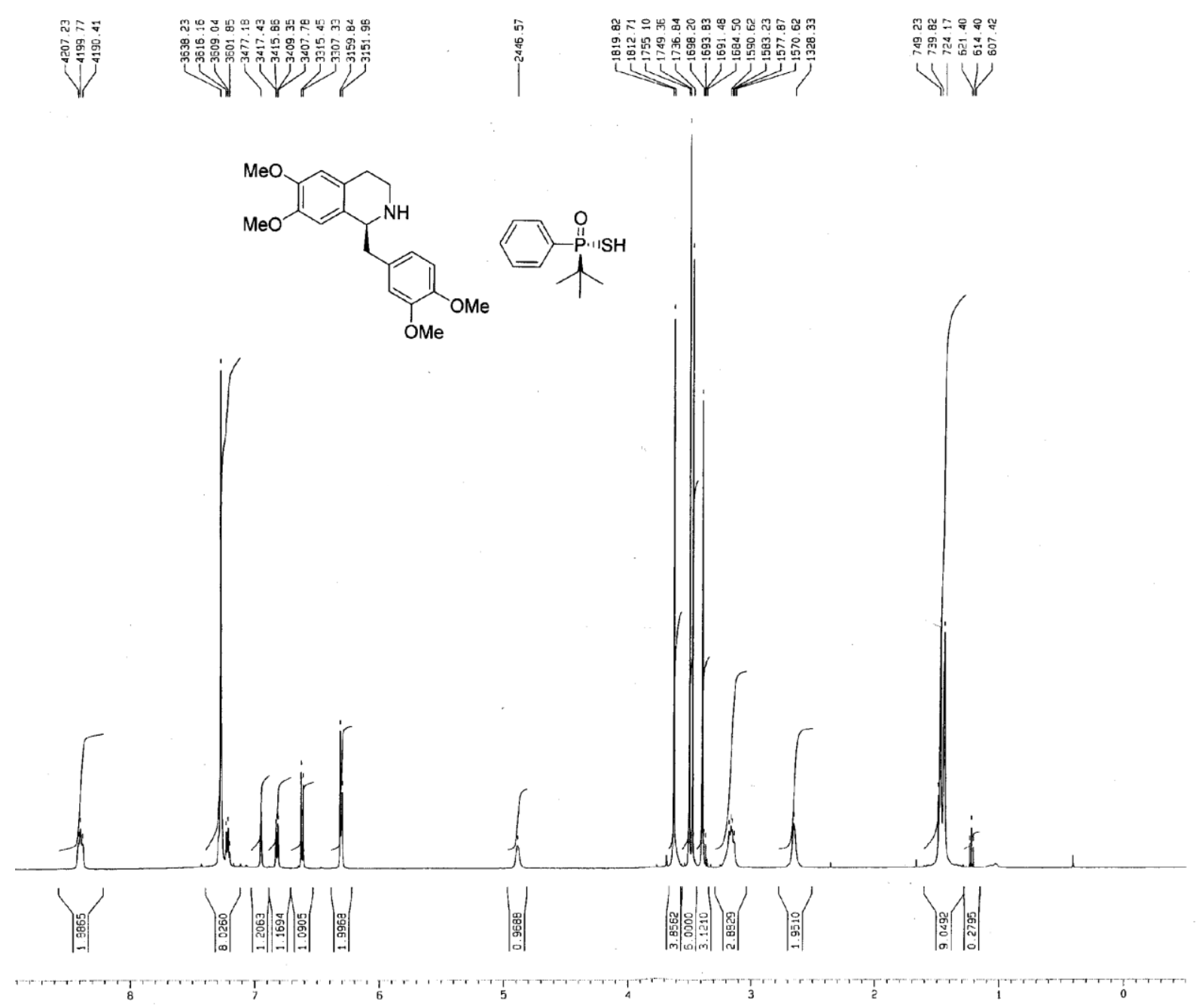

Figure S 176. ${ }^{1} \mathrm{H}$ NMR $\left(\mathrm{C}_{6} \mathrm{D}_{6}, 500 \mathrm{MHz}\right)$ spectrum of (-)-nor-laudanosine (99:1 er) with (+)-tertbutylphenylphosphinothioic acid. $(\gamma=1.05)$. 


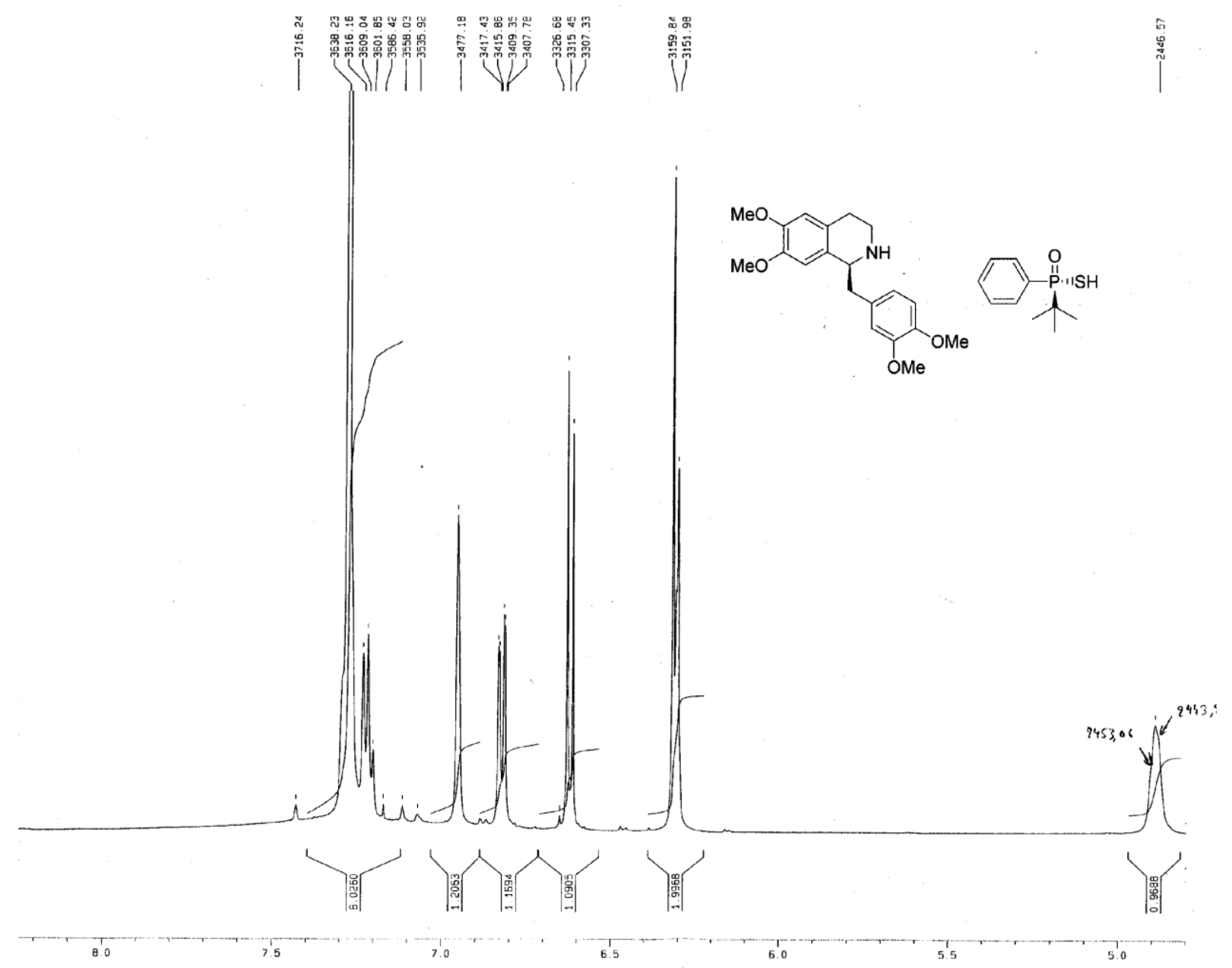

Figure $\mathrm{S}$ 177. ${ }^{1} \mathrm{H}$ NMR $\left(\mathrm{C}_{6} \mathrm{D}_{6}, 500 \mathrm{MHz}\right)$ spectrum of (-)-nor-laudanosine (99:1 er) with (+)-tertbutylphenylphosphinothioic acid. $(\gamma=1.05)$. 


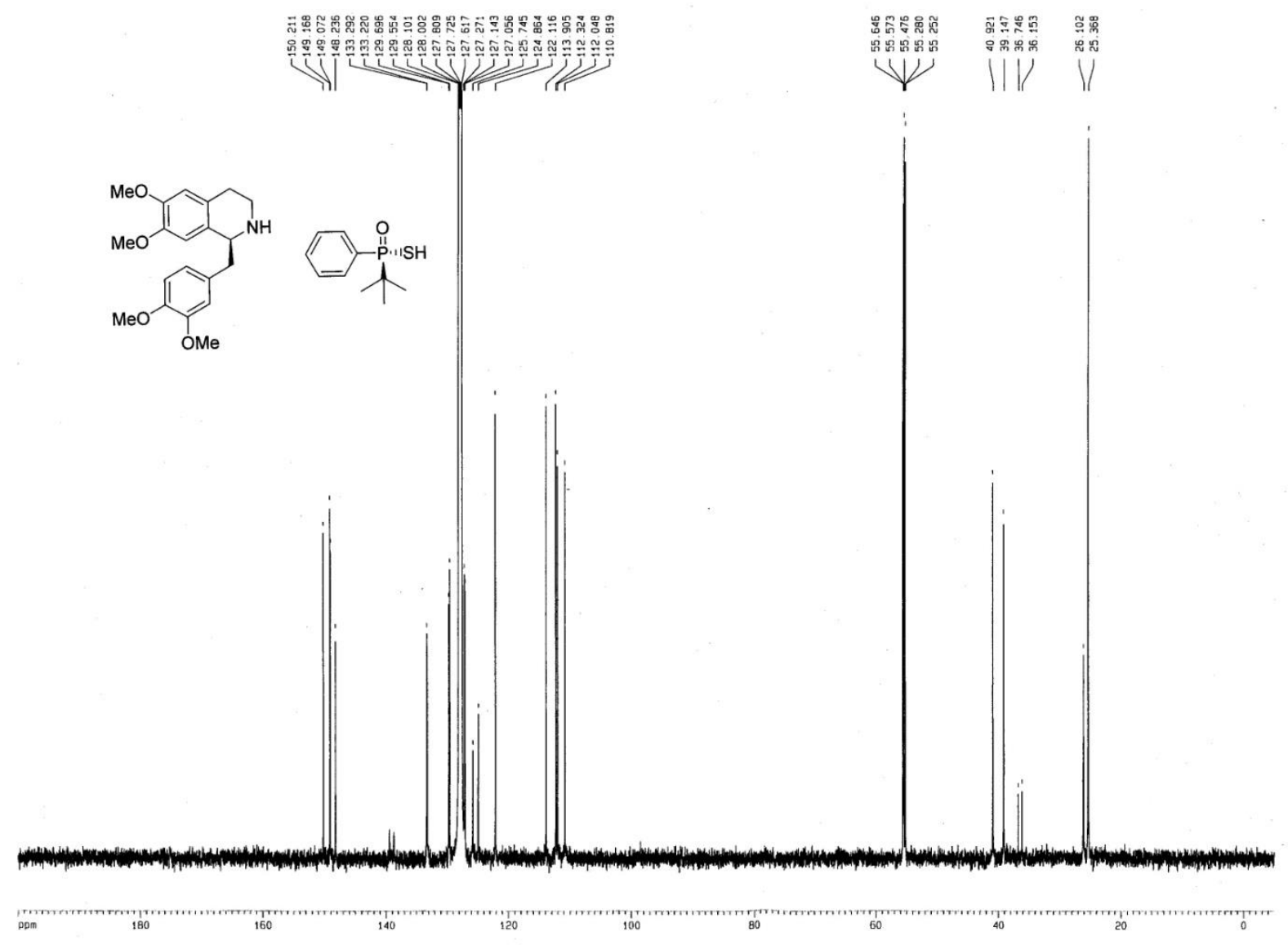

Figure S 178. ${ }^{13} \mathrm{C}$ NMR $\left(\mathrm{C}_{6} \mathrm{D}_{6}, 125 \mathrm{MHz}\right)$ spectrum of (-)-nor-laudanosine (99:1 er) with (+)-tertbutylphenylphosphinothioic acid. $(\gamma=1.05)$. 


\section{S181}

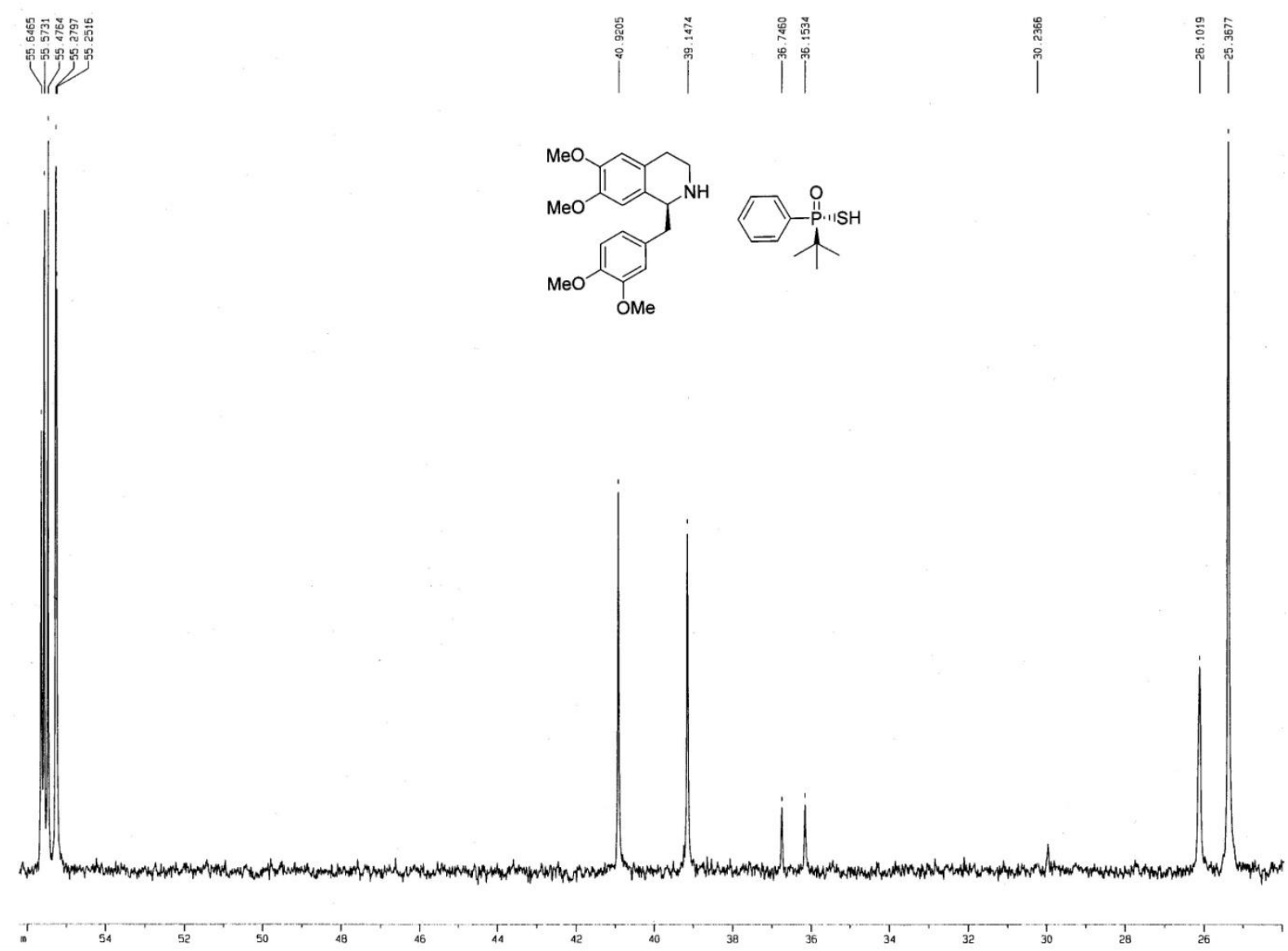

Figure S 179. ${ }^{13} \mathrm{C}$ NMR $\left(\mathrm{C}_{6} \mathrm{D}_{6}, 125 \mathrm{MHz}\right)$ spectrum of (-)-nor-laudanosine (99:1 er) with (+)-tertbutylphenylphosphinothioic acid. $(\gamma=1.05)$. Splitting signals are indicated with an asterisk $\left(^{*}\right)$. 
<smiles>COc1ccc(CC2NCCc3cc(OC)c(OC)cc32)cc1OC</smiles>

rac-norlaudanosine $\bullet(+)-14$.

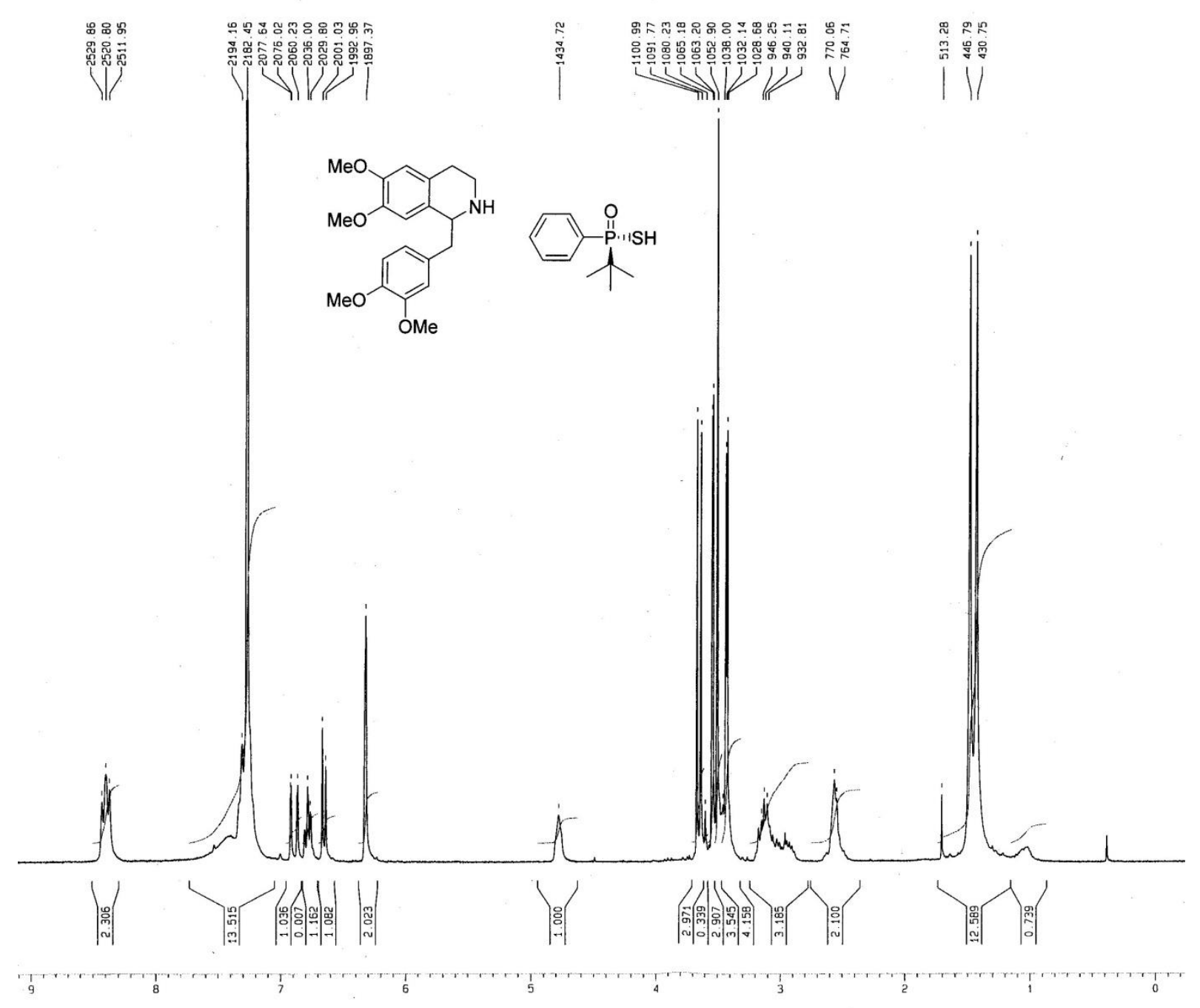

Figure S 180. ${ }^{1} \mathrm{H} \quad \mathrm{NMR}\left(\mathrm{C}_{6} \mathrm{D}_{6}, 300 \mathrm{MHz}\right)$ spectrum of rac-nor-laudanosine with $(+)$-tertbutylphenylphosphinothioic acid. $(\gamma=1.33)$. 


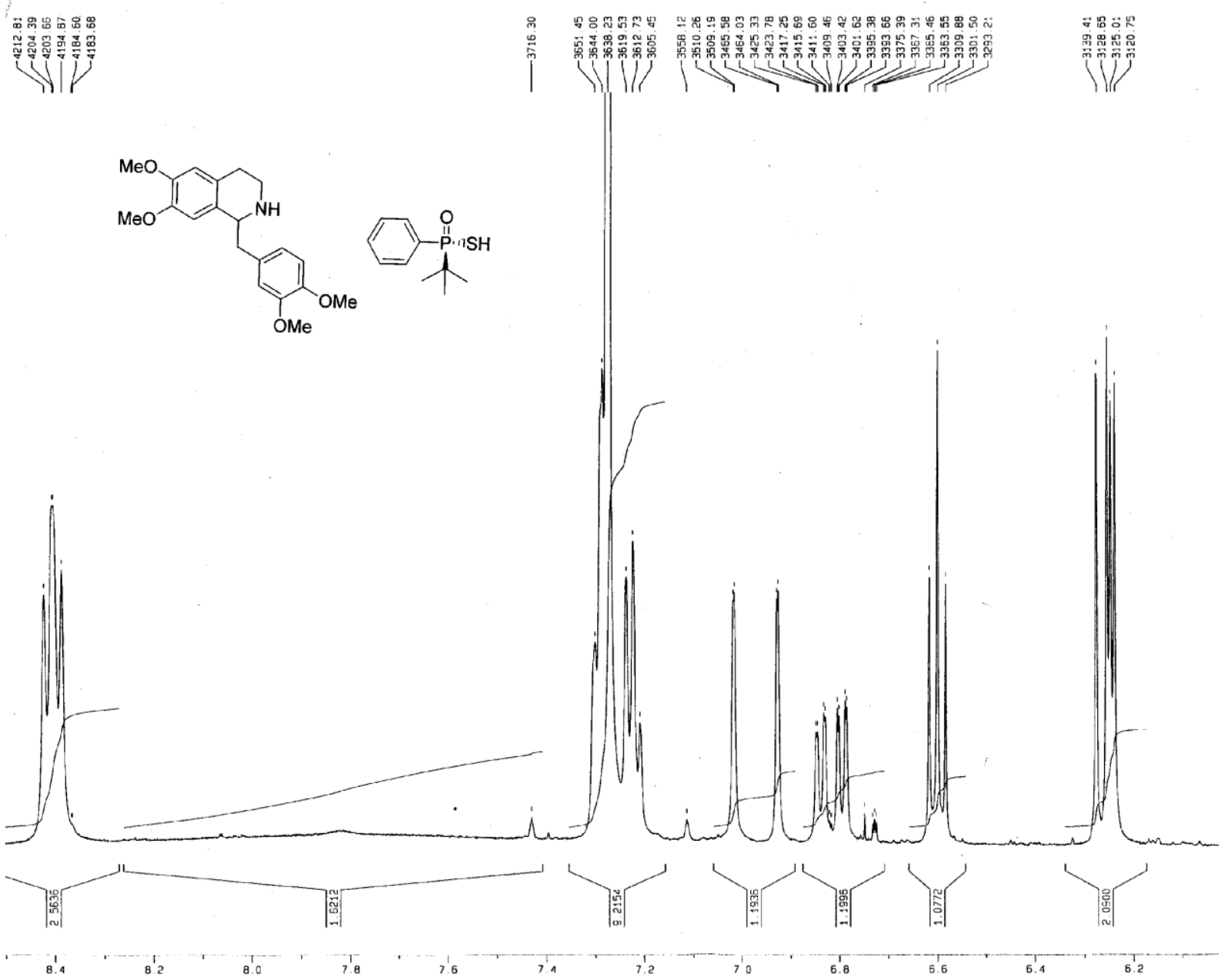

Figure S 181. ${ }^{13} \mathrm{C}$ NMR $\left(\mathrm{C}_{6} \mathrm{D}_{6}, 75 \mathrm{MHz}\right)$ spectrum of rac-nor-laudanosine with $(+)$-tertbutylphenylphosphinothioic acid. $(\gamma=1.33)$. 


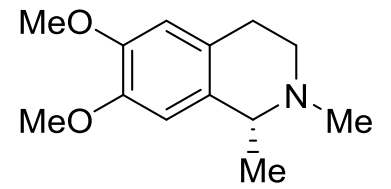

$(R)-(+)$-carnegine.

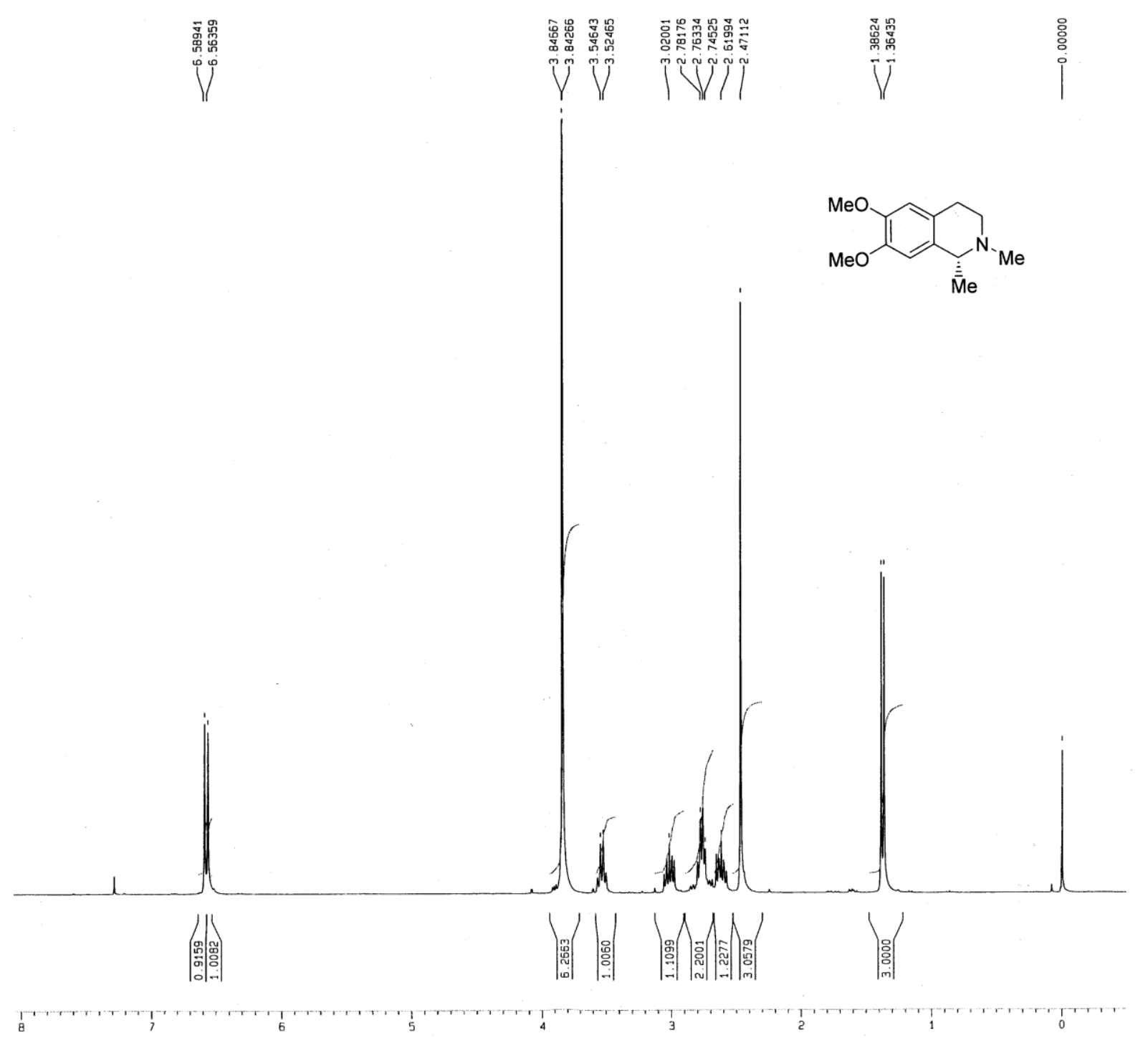

Figure S 182. ${ }^{1} \mathrm{H}$ NMR $\left(\mathrm{CDCl}_{3}, 300 \mathrm{MHz}\right)$ spectrum of (+)-carnegine. 


†

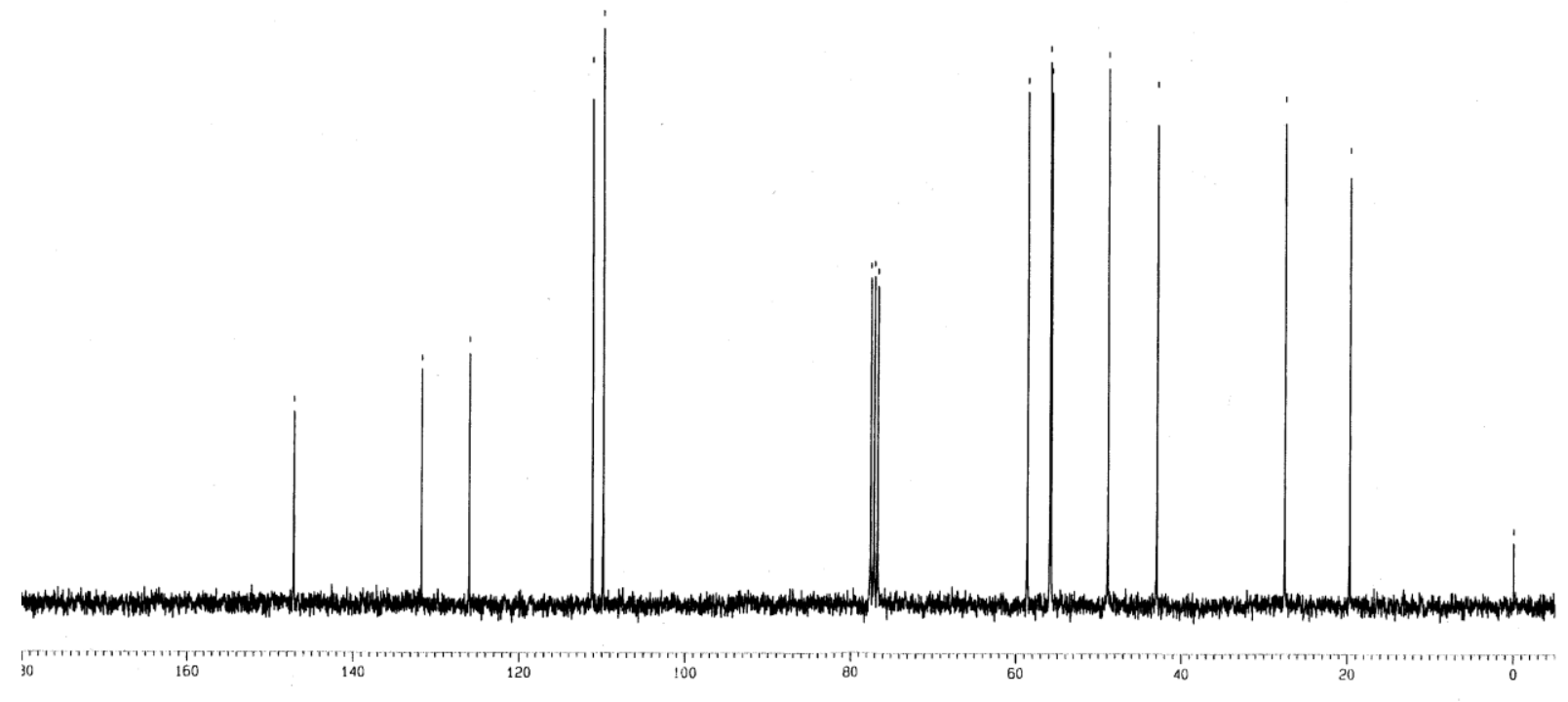

Figure S $183 .{ }^{13} \mathrm{C} \mathrm{NMR}\left(\mathrm{CDCl}_{3}, 75 \mathrm{MHz}\right)$ spectrum of (+)-carnegine. 


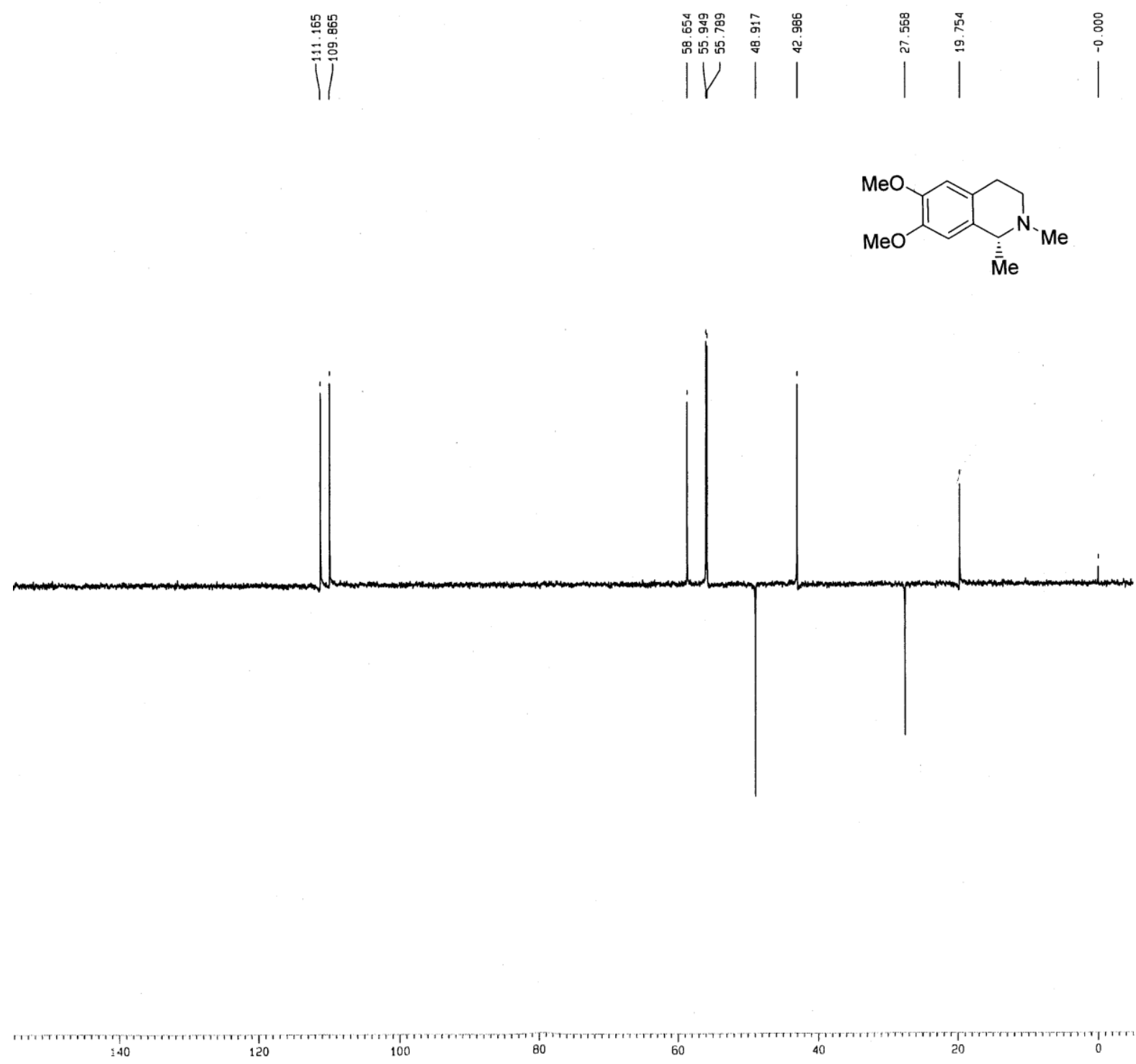

Figure S 184. DEPT C-NMR spectrum $\left(\mathrm{CDCl}_{3}, 75 \mathrm{MHz}\right)$ spectrum of $(+)$-carnegine. 


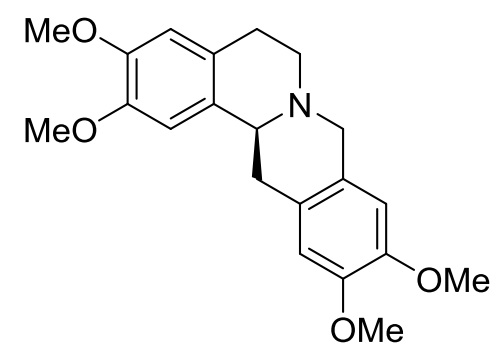

(S)-(-)-xylopinine.

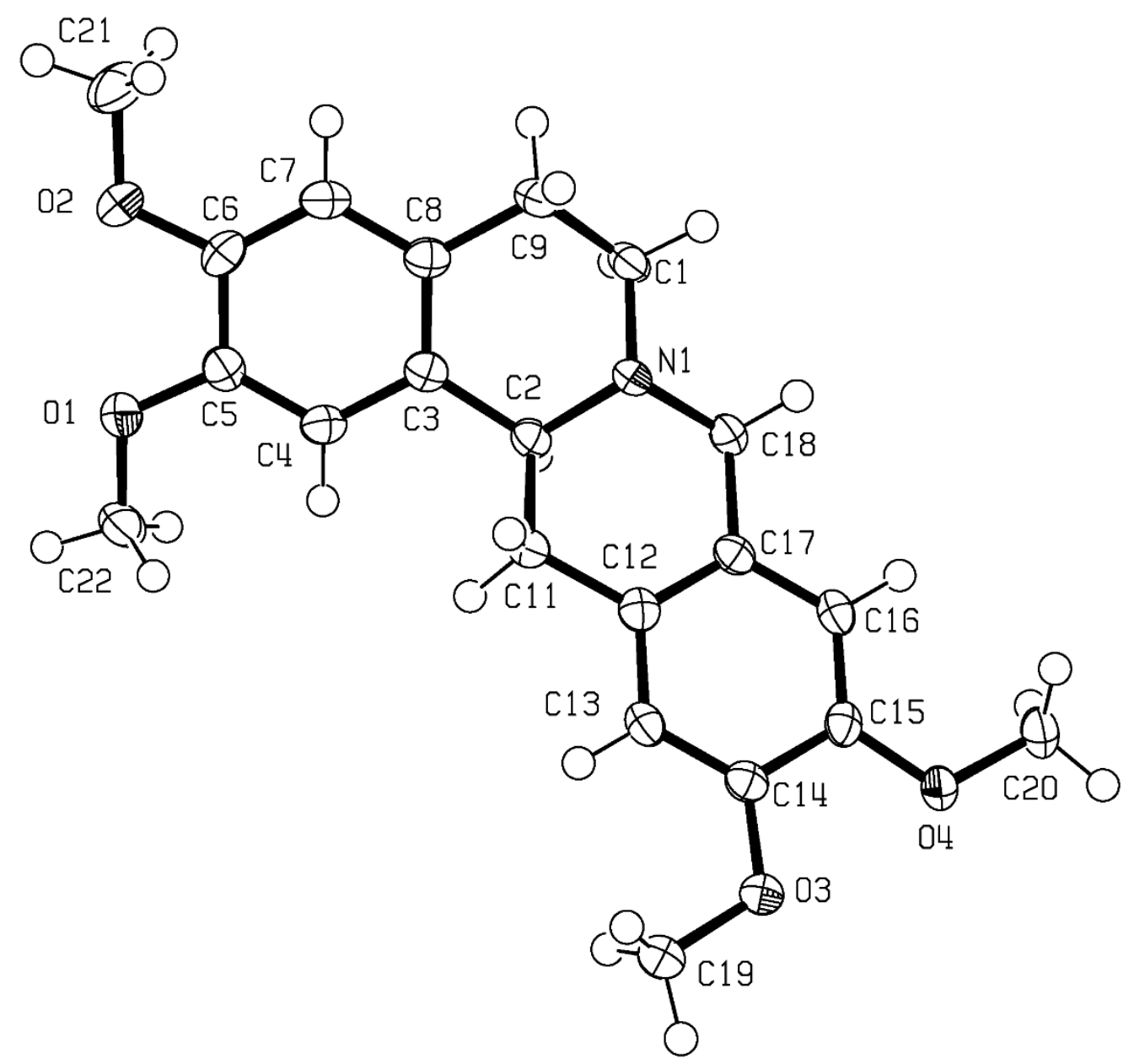

Figure $\mathbf{S}$ 185. ORTEP drawing of $(S)-(-)$-xylopinine. Ellipsoid plots are drawn with $70 \%$ of probability. 


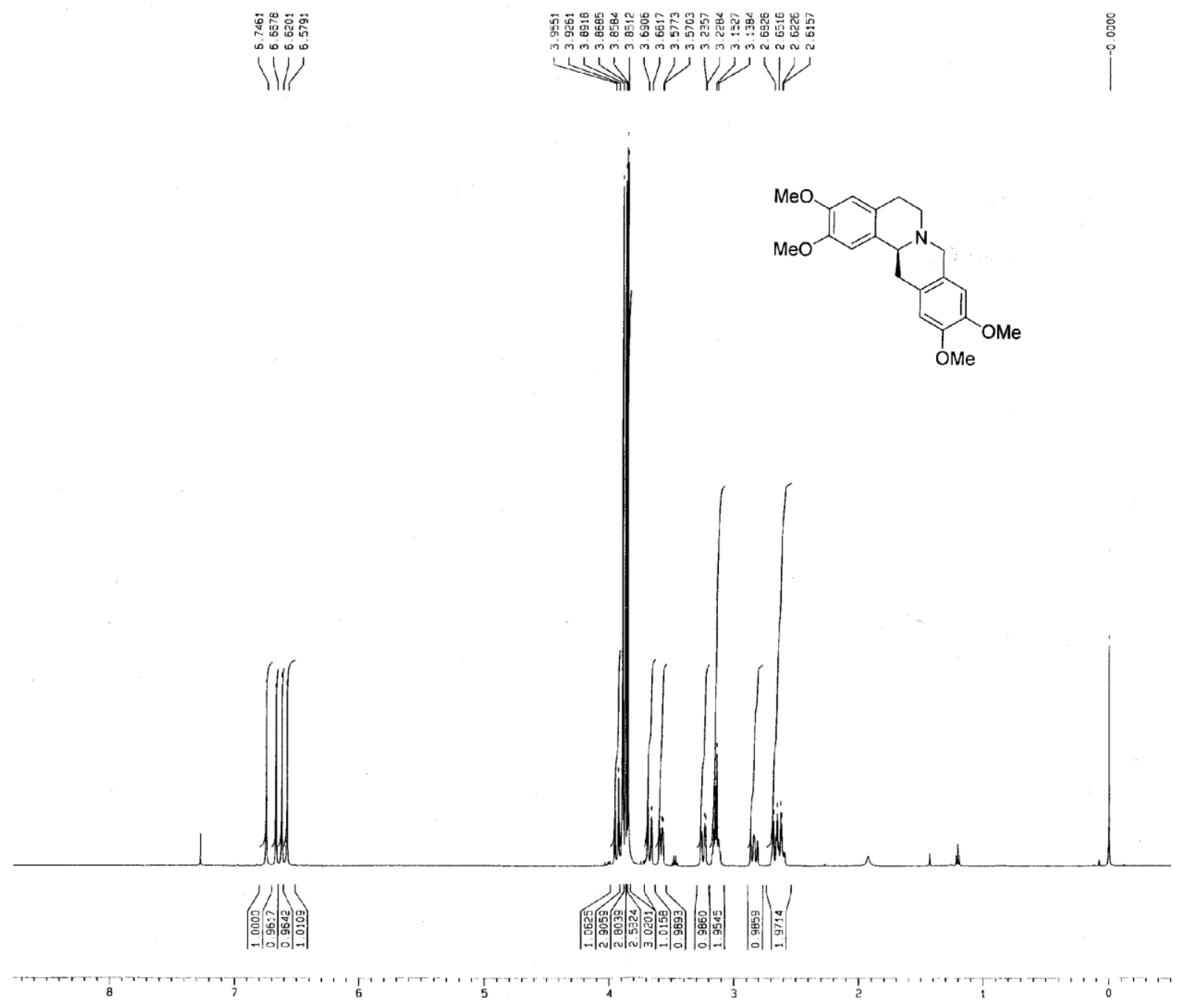

Figure S 186. ${ }^{1} \mathrm{H} \mathrm{NMR}\left(\mathrm{CDCl}_{3}, 500 \mathrm{MHz}\right)$ spectrum of $(-)$-xylopinine. 


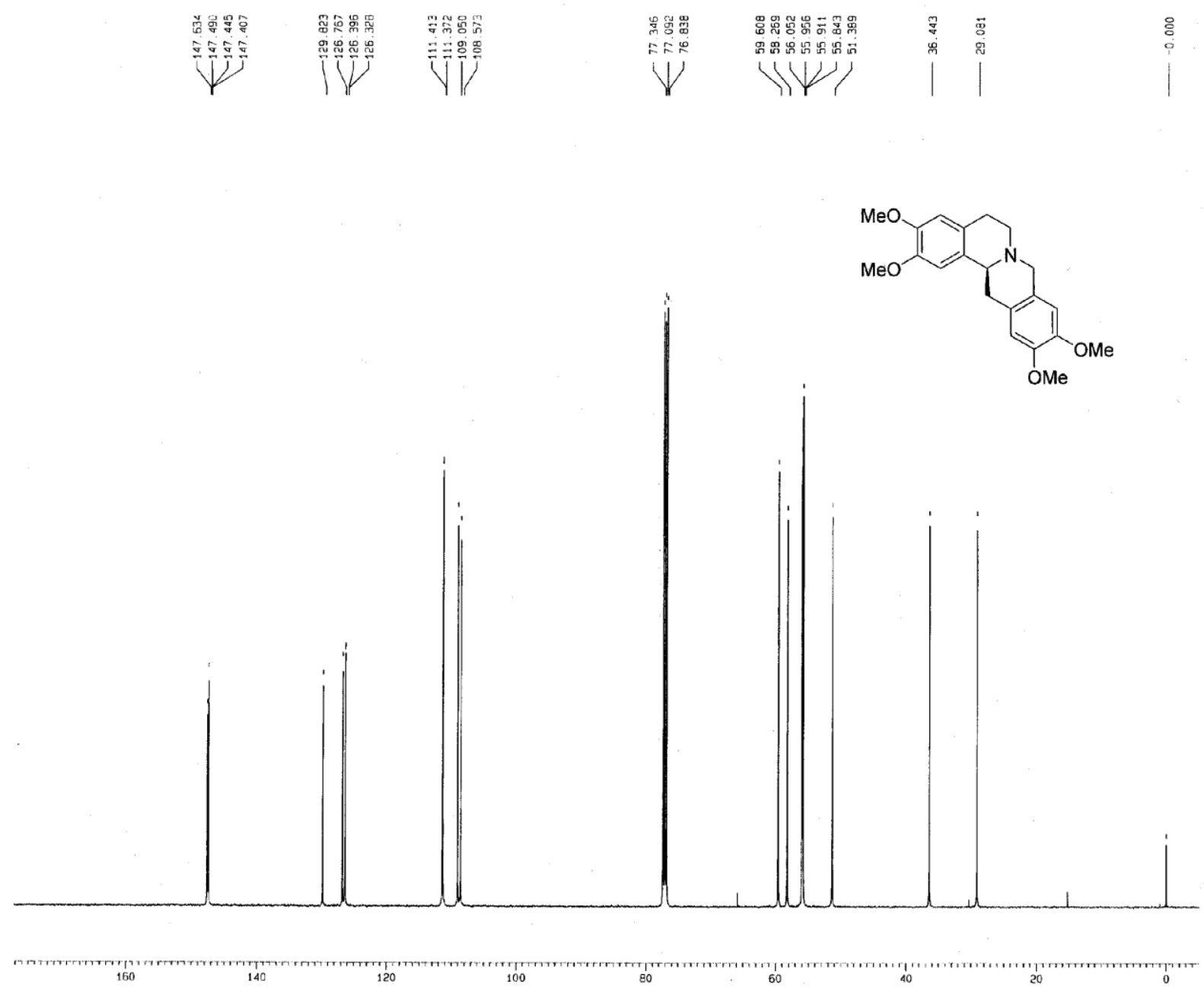

Figure S 187. $\left.{ }^{13} \mathrm{C} \mathrm{NMR} \mathrm{(CDCl}, 125 \mathrm{MHz}\right)$ spectrum of (-)-xylopinine. 


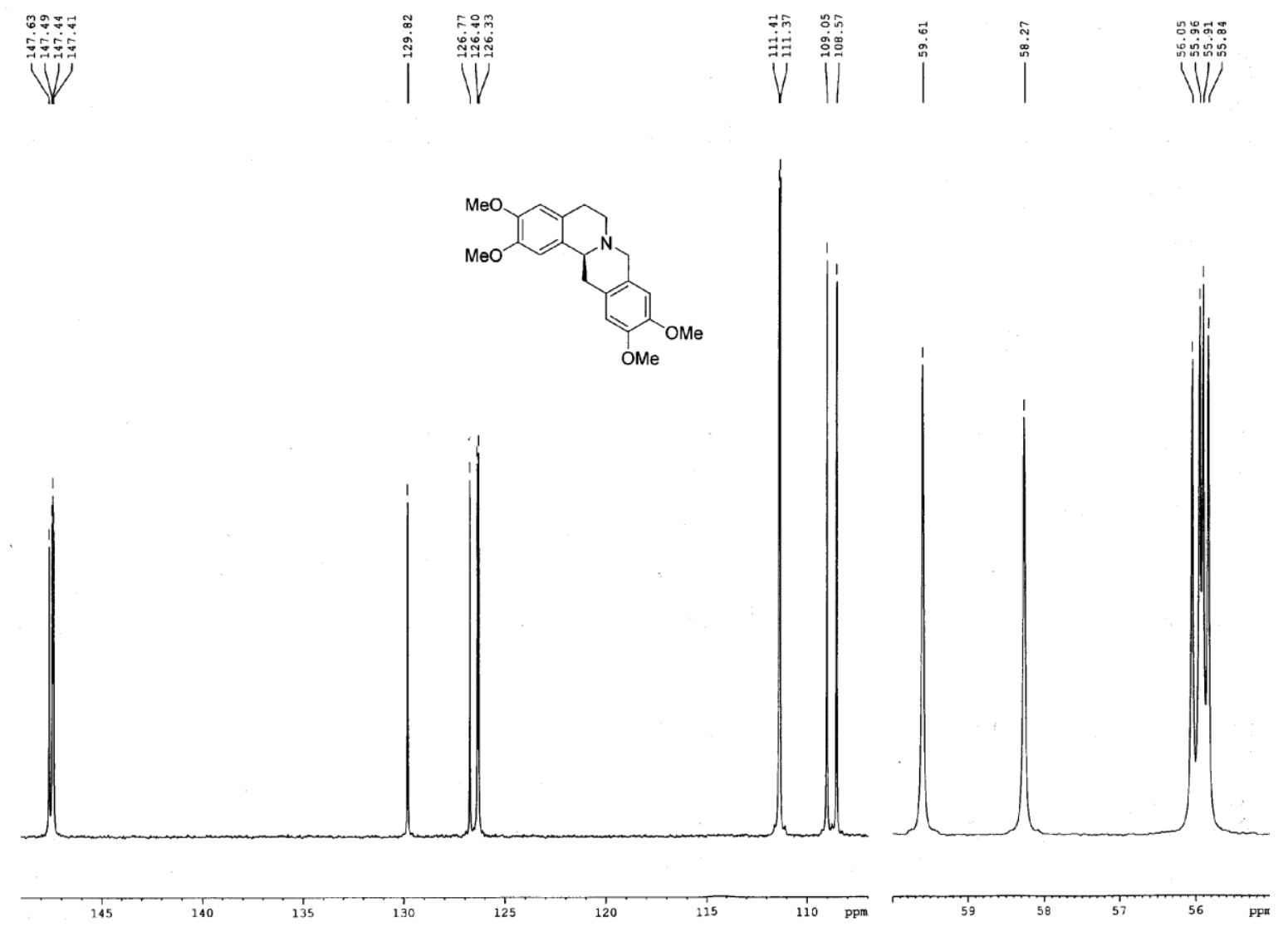

Figure $\mathbf{S} 188 .{ }^{13} \mathrm{C} \mathrm{NMR}\left(\mathrm{CDCl}_{3}, 125 \mathrm{MHz}\right)$ spectrum of (-)-xylopinine. 
S191

$$
\text { 麗 }
$$
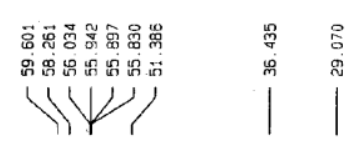

i

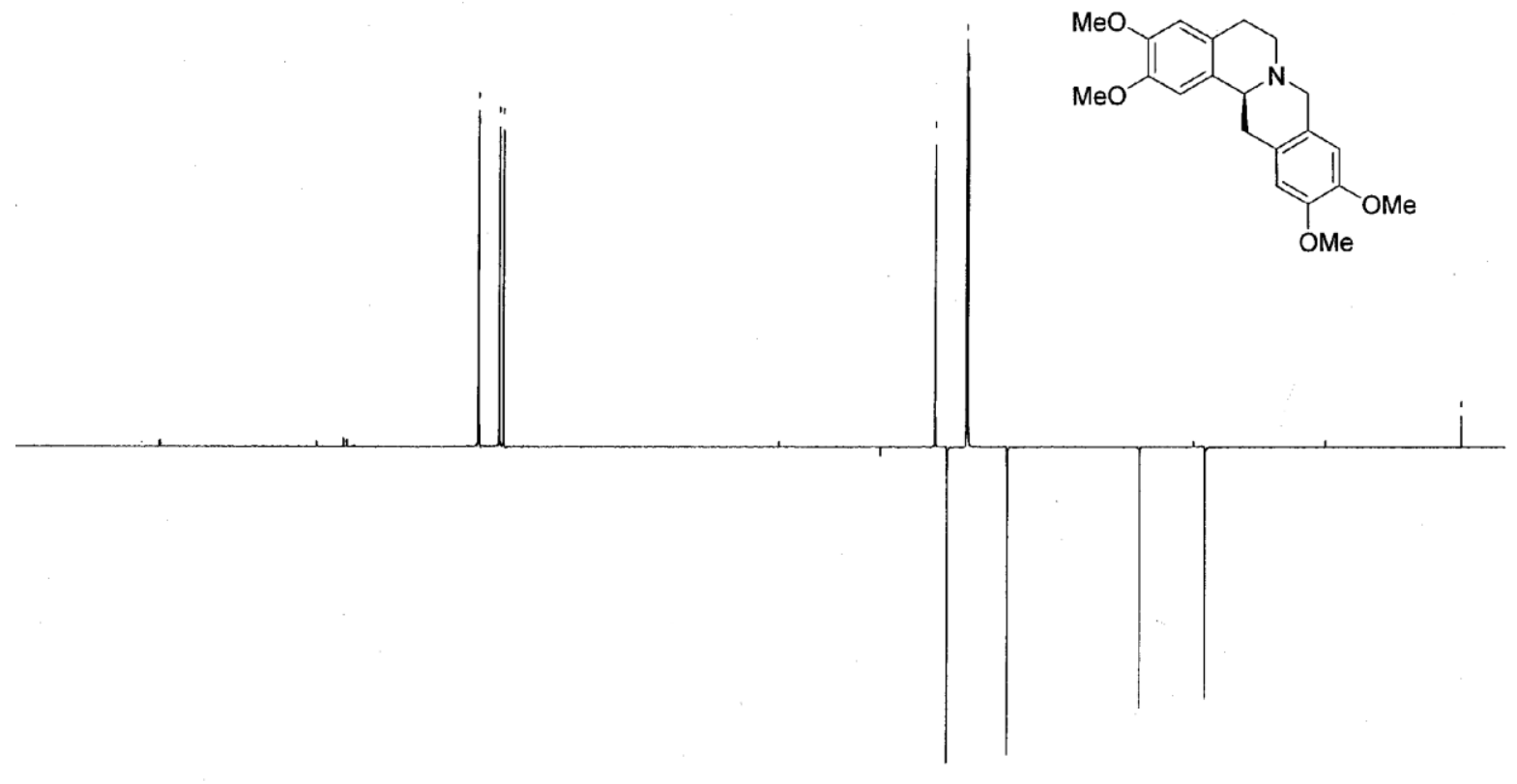

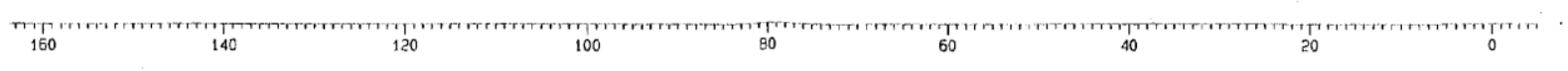

Figure S 189. DEPT C-NMR spectrum ( $\left.\mathrm{CDCl}_{3}, 125 \mathrm{MHz}\right)$ of $(-)$-xylopinine. 